\title{
Utilizing the coughing rat model of pertussis to improve vaccine efficacy
}

Jesse M. Hall

West Virginia University, jmh0059@mix.wvu.edu

Follow this and additional works at: https://researchrepository.wvu.edu/etd

Part of the Bacteria Commons, Medical Immunology Commons, and the Medical Microbiology

\section{Commons}

\section{Recommended Citation}

Hall, Jesse M., "Utilizing the coughing rat model of pertussis to improve vaccine efficacy" (2021).

Graduate Theses, Dissertations, and Problem Reports. 8269.

https://researchrepository.wvu.edu/etd/8269

This Dissertation is protected by copyright and/or related rights. It has been brought to you by the The Research Repository @ WVU with permission from the rights-holder(s). You are free to use this Dissertation in any way that is permitted by the copyright and related rights legislation that applies to your use. For other uses you must obtain permission from the rights-holder(s) directly, unless additional rights are indicated by a Creative Commons license in the record and/ or on the work itself. This Dissertation has been accepted for inclusion in WVU Graduate Theses, Dissertations, and Problem Reports collection by an authorized administrator of The Research Repository @ WVU.

For more information, please contact researchrepository@mail.wvu.edu. 
Graduate Theses, Dissertations, and Problem Reports

2021

Utilizing the coughing rat model of pertussis to improve vaccine efficacy

Jesse M. Hall

Follow this and additional works at: https://researchrepository.wvu.edu/etd

Part of the Bacteria Commons, Medical Immunology Commons, and the Medical Microbiology Commons 
Utilizing the coughing rat model of pertussis to improve vaccine efficacy.

$$
\text { Jesse Michael Hall }
$$

\title{
Dissertation submitted to the School of Medicine at West Virginia University
}

\author{
in partial fulfillment of the requirements \\ for the degree of \\ Doctor of Philosophy in \\ Immunology and Microbial Pathogenesis \\ F. Heath Damron, Ph.D., Committee Chair \\ Joseph Horzempa, Ph. D. \\ Edwin Wan, Ph. D. \\ Cory Robinson, Ph. D. \\ Tim Eubank, Ph. D.
}

\begin{abstract}
Department of Microbiology, Immunology, and Cell Biology
Morgantown, West Virginia
\end{abstract}

2021

Keywords: Bordetella pertussis, pertussis, DTaP, mucosal immunization, whole body plethysmography, coughing rat model of pertussis

Copyright 2021 Jesse M Hall 


\section{Abstract}

\section{Utilizing the coughing rat model of pertussis to improve vaccine efficacy.}

\section{Jesse Michael Hall}

Pertussis, also known as whooping cough, is a respiratory disease caused by the highly contagious, Gram-negative pathogen Bordetella pertussis (Bp). Infection occurs through inhalation of aerosolized droplets containing $B p$, which then colonizes ciliated epithelial cells of the respiratory tract. Here, $B p$ expresses toxins and virulence factors that lead to leukocyte recruitment, paroxysmal cough, and impairment of host innate responses. Currently, in developed countries, acellular pertussis vaccines (aP; DTaP; Tdap) are used to prevent $B p$ infection and whooping cough disease. However, we currently realize that the aP vaccine efficacy quickly wanes resulting in a reemergence of pertussis. Recent work performed by the CDC illustrates that current strains are genetically divergent from the strains originally used to formulate the aP, which may be partially responsible for the short-lived aP immunity. In an effort to evaluate pathogenesis of recent circulating strains and, in turn, improve vaccine efficacy, an animal model that recapitulates clinical manifestations of pertussis is necessary. First, we reintroduced the coughing rat model of pertussis by comparing two genetically divergent strains, Tohama 1 and recent isolate D420. Seven-week-old Sprague-Dawley rats were intranasally (IN) challenged with $10^{8}$ viable CFUs. We measured the hallmark signs of pertussis disease such as neutrophilia, pulmonary inflammation, paroxysmal cough using whole body plethysmography (WBP), bacterial burden of the respiratory tract and characterized the serological response to known virulence factors. Overall, rats infected with D420 had an increase in bacterial 
burden in the lung and nasal cavity, an increase in coughs over the course of infection, and an increase in anti-Bp IgM antibody titers compared to Tohama 1 challenged rats. The coughing rat model of pertussis can further be used as a preclinical tool to evaluate vaccine efficacy, as they are more feasible than baboons while still demonstrating classical manifestations of the disease. Next, we evaluated vaccine mediated immunity induced through IN and oral gavage (OG) vaccination of DTaP in the coughing rat model of pertussis, compared to intramuscular (IM)-whole cell pertussis (wP) and IM-aP immunized rats. Following vaccination, rats were similarly IN infected with $10^{8} \mathrm{CFUs}$ of $B p$ strain D420, cough, bacterial burden, respiratory distress, and both systemic and mucosal serological responses were analyzed over the nine-day infection. Our data show that both IN and OG vaccination protected against $B p$ colonization in the respiratory tract and $B p$ induced cough, similarly to IM-wP and IM-aP vaccinated rats. IN-aP and OG-aP vaccination also resulted in the production of anti- $B p \operatorname{lgG}$ antibody titers at day 9 postchallenge; additionally, IN-aP vaccination induced mucosal anti-Bp IgA antibodies in the nasal cavity. Histology confirmed that both IN-aP and OG-aP immunization protected against acute inflammation in the lung. Altogether, these data further support that mucosal vaccination can generate a protective immune response against pertussis. Furthermore, the coughing rat model of pertussis can be used to improve our understanding of $B p$ pathogenesis and can be used to evaluate the "next generation" of pertussis vaccines. 


\section{Dedication}

The work presented here is dedicated to my parents John and Dianne Hall. To the endless sacrifices you have made for me, I am forever grateful.

To my wife Samantha, Felix, and God who has provided me with unwavering support and love.

To my great grandfather John P. Howard

To anyone who believes that they too can make a difference. 


\section{Acknowledgements}

First, I would like to thank my advisor Dr. F. Heath Damron for continually pushing me to be a better version of myself each day. I am grateful for the opportunity to join your research program and grow into the scientist I am today. I would also like to thank Dr. Mariette Barbier for spending so many hours helping me design, analyze, and artistically craft beautiful figures and presentations.

To the DB lab family, each one of you have had a part in shaping me into the person that I am today. To current and past members, I would like to thank Dr. Dylan Bohem, Dr. Emel Sen-Kilic, William Witt, Ting Wong, Kelly Weaver, Gage Pyles, Graham Bitzer, Dr. Allison Wolf, Dr. Catherine Blackwood, Megan DeJong, Jeanna Blake, Justin Bevere, Annalisa Huckaby, Aaron Malkowski, Dr. Jason Kang, Dr. Alexander Horspool, and Dr. Brynnan Russ, Casey Cunningham, and Dr. Paz Gutierrez. I wish you all bright and illustrious careers ahead.

I would also like to thank my dissertation committee. Thank you for being the compass guiding me along my scientific journey. The knowledge and mentorship you have instilled in me will never be forgotten.

I also want to say thank everyone in the Microbiology, Immunology, and Cell Biology department. I specifically want to thank Dr. Kathleen Brundage, Dr. Karen Martin, and Dr. Amanda Ammer. I cannot thank you all enough for all the help and guidance you all provided me over my graduate career.

Lastly, I want to thank my family, my friends, and my wife. I would not be here today if it was not for all the love and support. You all have been my rock and the driving force pushing me to who I am today. I am absolutely blessed and privileged to have you all in my life

-Thank you 


\section{Table of Contents}

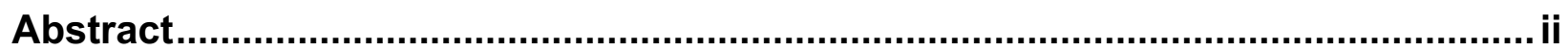

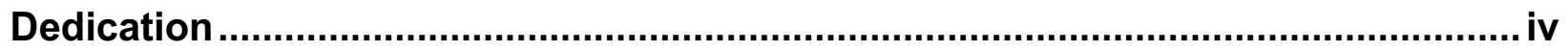

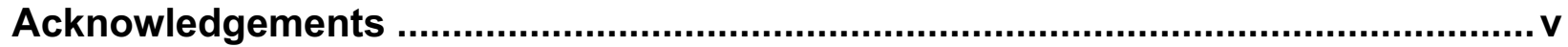

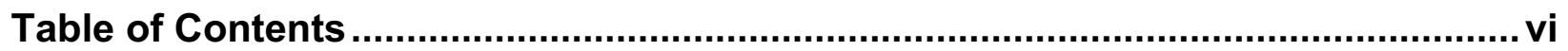

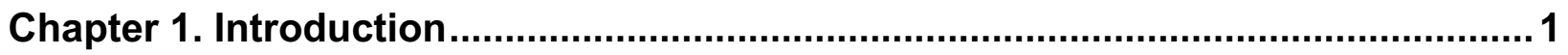

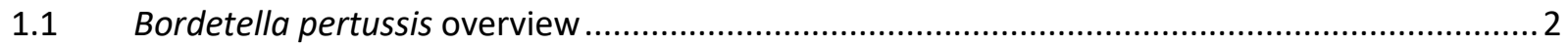

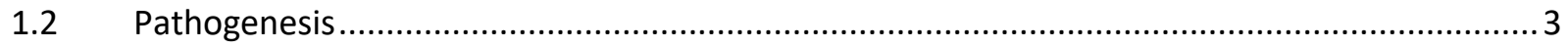

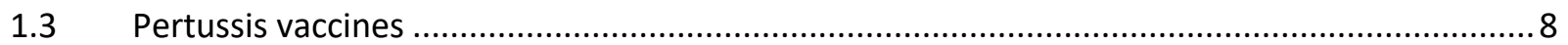

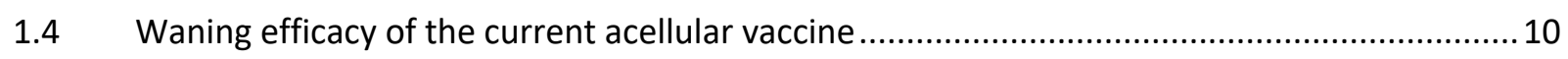

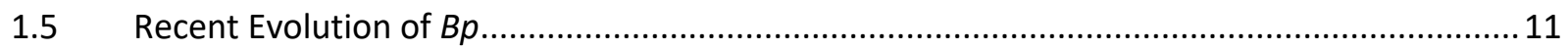

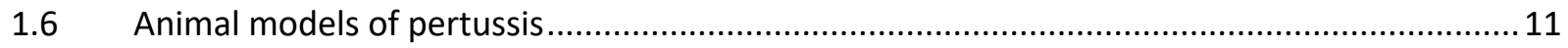

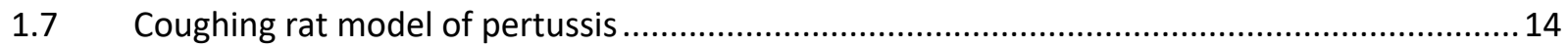

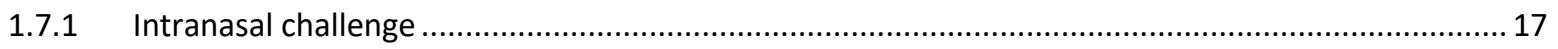

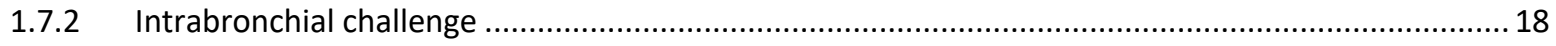

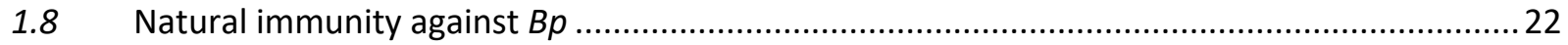

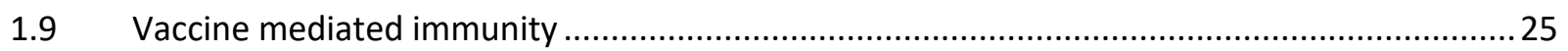

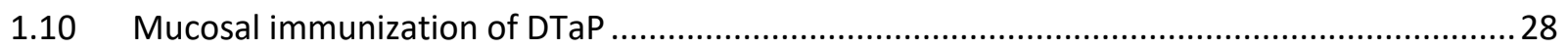

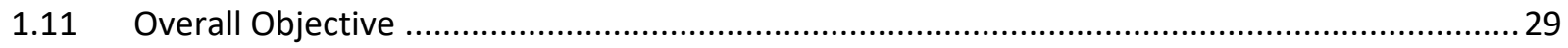

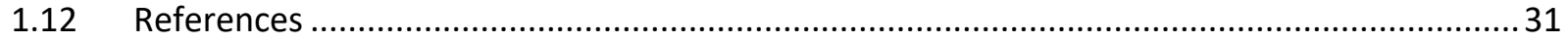

Chapter 2. Re-investigating the coughing rat model of pertussis to understand Bordetella pertussis pathogenesis ........................................................................51

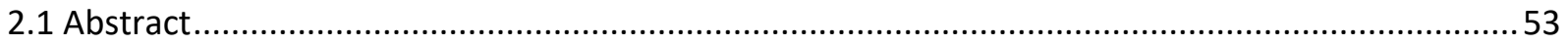

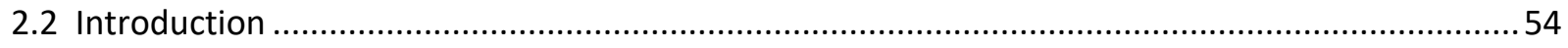


2.3 Results.

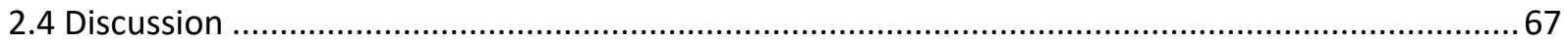

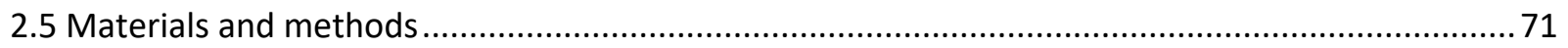

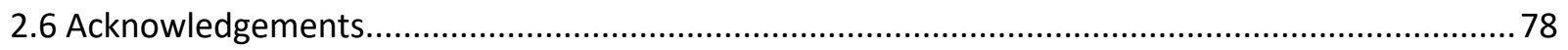

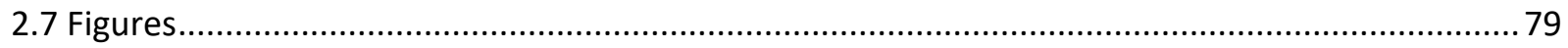

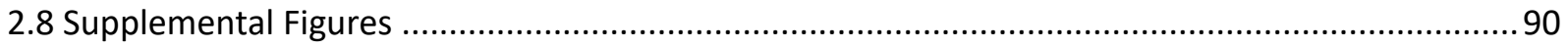

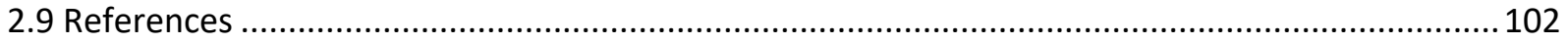

\section{Chapter 3. Mucosal immunization with DTaP confers protection against}

Bordetella pertussis infection and cough in Sprague-Dawley rats.......................113

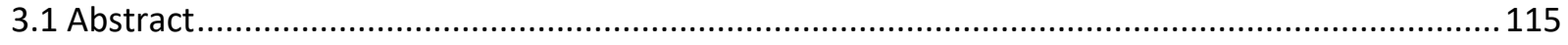

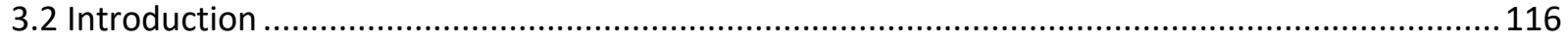

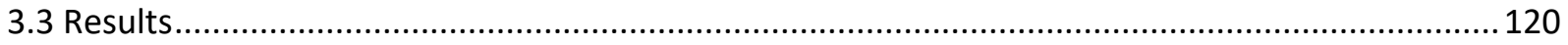

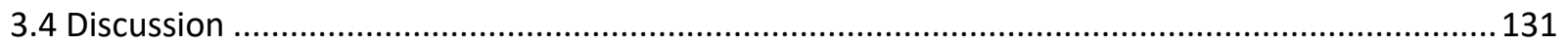

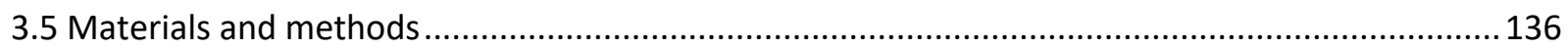

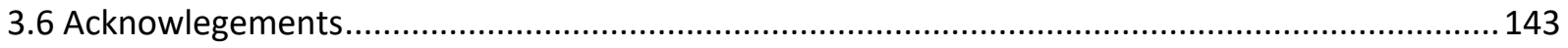

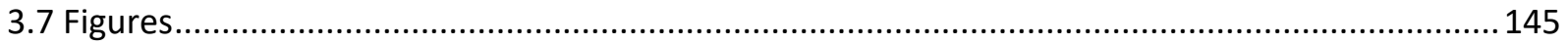

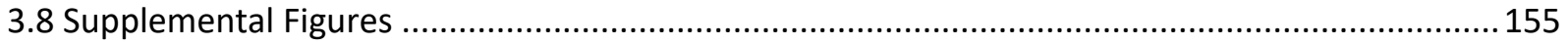

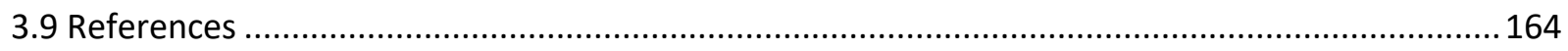

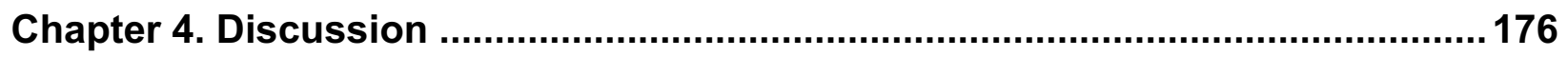

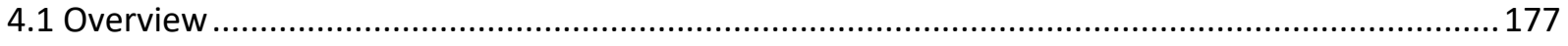

4.2 Lessons learned from the coughing rat model of pertussis .....................................................178

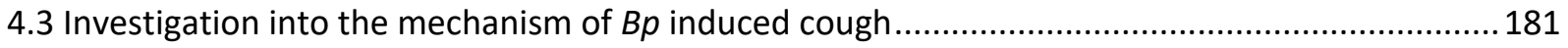

4.4 Bordetella bronchiseptica and Bordetella parapertussis challenge experiments in rats ..............182

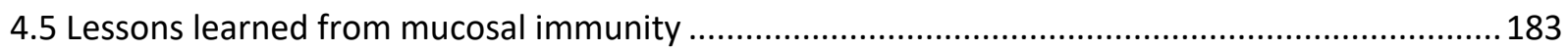

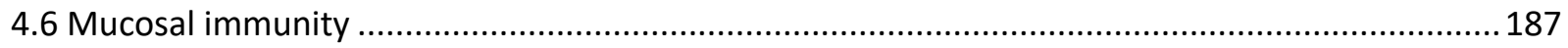


4.7 Improvements to DTaP/Tdap 188

4.8 Improvement of oral vaccination against pertussis 191

4.9 Experimental oral vaccines of potential.. 192

4.10 In summary 193

4.11 References

194

\section{List of Figures}

Figure 1. Reported pertussis incidence in the United States from 1922-2019...............9

Figure 2. Percent death of young $B p$ infected rats............................................... 17

Figure 3. Summary of the immunity generated from natural infection. .........................25

Figure 4. Summary of the DTP and DTaP vaccine mediated immunity......................27

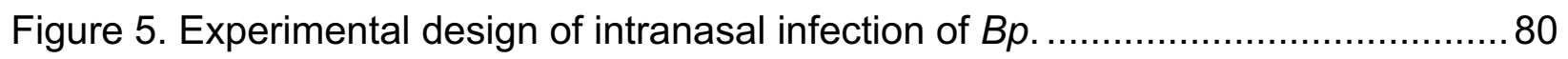

Figure 6. Infection of Bordetella pertussis induces cough in Sprague-Dawley rats. .......81

Figure 7. Bordetella pertussis infection impairs respiratory capacity. ......................... 82

Figure 8. Bp infection induces acute and chronic inflammation in the lung of SpragueDawley rats.

Figure 9. Bp infection induces inflammation of the lung and decrease in weight gain... 84

Figure 10. Analysis of the nasal associated lymphoid tissue. ................................... 85

Figure 11. Analysis of bacterial burden over the course of $B p$ infection. ..................... 86

Figure 12. Immunofluorescence staining of $B p$ localization in the respiratory tract. ...... 87

Figure 13. Neutrophil recruitment and proinflammatory cytokine production after challenge determined by flow cytometry and multiplex immunoassay analysis............. 88

Figure 14. Measurement of serum antibody titers over the course of $B$. pertussis infection. 
Figure 15. IN booster vaccination induces systemic anti-Bp and anti-PT antibody titers.

Figure 16. Intranasal and oral vaccination of acellular pertussis vaccine decreases cough of $B$. pertussis infected rats.

Figure 17. Intranasal vaccination decreases pulmonary restriction of Bordetella pertussis infected rats.

Figure 18. Mucosal vaccination induces production of anti- $B p$ IgG, while IN immunization also induces both anti-PT IgM and IgG antibodies. 148

Figure 19. Intranasal immunization elicits the production of anti-Bp $\lg A$ in the respiratory tract.

Figure 20. Mucosal vaccination protects against acute and total inflammation in the lung of $B p$ infected Sprague-Dawley rats. 150

Figure 21. Oral and intranasal immunization decreased B. pertussis bacterial burden in the respiratory tract. 152

Figure 22. Measurement of cytokines in the lung and serum at days 1 and 9 post infection. 153

Figure 23. Systemic and mucosal anti-Bp and anti-PT antibodies correlate with observed protection. 154

Figure 24. Vaccine generated immune responses from intramuscular and mucosal immunization of DTaP, and booster immunization of DTaP 189

\section{List of tables}

Table 1. Bordetella virulence associated genes. +/- annotates expression in select strains

Table 2. Summary of literature and results using the coughing rat model of pertussis.. 16 Table 3. Summary of vaccine mediated immune responses following $B p$ challenge in the coughing rat model of pertussis. 185 


\section{List of Supplemental figures}

Figure S 1. Set up for the whole-body plethysmograph ........................................... 90

Figure S 2. Diagram illustrating Penh during $B p$ infection........................................ 91

Figure S 3. Bordetella pertussis infection induces cellular recruitment in the lungs ......92

Figure S 4. Bacterial colonization comparisons between CD1 mice and Sprague-Dawley

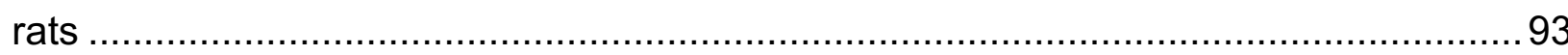

Figure S 5. Hematoxylin and eosin staining of the nasal cavity of Sprague-Dawley rat.94

Figure S 6. Immunofluorescence (IF) staining of control rat's respiratory tract.............. 95

Figure S 7. Serum cytokine response during twelve-day Bordetella pertussis infection 96

Figure S 8. Lung cytokine response during twelve-day Bordetella pertussis infection. . 97

Figure S 9. Serum chemokine during twelve-day Bordetella pertussis infection ...........98

Figure S 10. Lung chemokine during twelve-day Bordetella pertussis infection ............ 99

Figure $S$ 11. Measurement of serum antibody titers over the course of $B$. pertussis infection. 100

Figure $S$ 12. Area under the curve analysis of $\lg M$ and $\lg G$ serum antibody titers against Bordetella pertussis over the course of infection 101

Figure S 13. Experimental design of vaccination and challenge .............................. 155

Figure S 14. Respiratory profile of Sprague-Dawley rats vaccinated and challenged with B. pertussis. 156

Figure $S$ 15. ELISpot assay was performed to measure the number of $B p$ specific IgG cells from the bone marrow at day 9 post challenge 158

Figure S 16. Measurement of lung and body weight at days 1 and 9 post challenge.. 159 Figure S 17. Measurement of cytokines in the lung at days 1 and 9 post infection ..... 160 Figure $S$ 18. Measurement of cytokines in the serum at days 1 and 9 post infection. . 161 Figure S 19. Characterizing circulating cell populations in the blood and lung post infection. 162 
Figure S 20. Proposed mucosal immune response induced through oral vaccination. 163

\section{List of abbreviations}

$\begin{array}{ll}\text { ACIP } & \text { Advisory Committee on Immunization Practices } \\ \text { ACT } & \text { Adenylate cyclase toxin } \\ \text { ANOVA } & \text { Analysis of variance } \\ \text { aP, DTaP,Tdap } & \text { Acellular pertussis vaccine } \\ \text { AUC } & \text { Area under the curve } \\ \text { Bb } & \text { Bordetella bronchiceptica } \\ \text { BG } & \text { Bordet-Gengou } \\ \text { Bp } & \text { Bordetella pertussis } \\ \text { BrkA } & \text { Bordetella resistance to killing A } \\ \text { BSA } & \text { Bovine Serum Albumin } \\ \text { CDC } & \text { Centers of Disease Control } \\ \text { CoP } & \text { Correlates of protection } \\ \text { DTP } & \text { Diphtheria Tetanus Pertussis vaccine } \\ \text { EDTA } & \text { Ethylenediaminetetraacetic acid } \\ \text { ELISA } & \text { Enzyme-linked immunosorbent assay } \\ \text { ELISpot } & \text { Enzyme-linked immune absorbent spot assay } \\ \text { F } & \text { Frequency } \\ \text { FHA } & \text { Filamentous hemagglutinin } \\ \text { Fim } & \text { Fimbriae } \\ \text { GALT } & \text { Gut-associated lymphoid tissue } \\ \text { H\&E } & \text { A }\end{array}$




\begin{tabular}{|c|c|}
\hline IACUC & Institutional Animal Care and Use Committee \\
\hline IB & Intrabronchial \\
\hline IC & Intracerebral challenge \\
\hline IF & Immunofluorescence \\
\hline IM & Intramuscular \\
\hline IN & Intranasal \\
\hline IP & Intraperitoneal \\
\hline LOS & Lipooligosaccharide \\
\hline LPS & Lipopolysaccharide \\
\hline MPLA & Monophosphoryl lipid-A \\
\hline $\mathrm{MVb}$ & Minute Volume \\
\hline MVC & Mock vaccinated challenge \\
\hline NALT & Nasal-associated lymphoid tissue \\
\hline NETs & Neutrophil extracellular traps \\
\hline OG & Oral Gavage \\
\hline PAU & Pause \\
\hline PBS & Phosphate buffer Saline \\
\hline PEF & Peak expiratory height \\
\hline PenH & Enhanced pause \\
\hline PIF & Peak inspiratory height \\
\hline PMNs & Polymorphonuclear cells \\
\hline PRN & Pertactin \\
\hline PT & Pertussis toxin \\
\hline
\end{tabular}




$\begin{array}{ll}\text { PTd } & \text { Detoxified pertussis toxin } \\ \text { RTX } & \text { Repeat in toxin domain } \\ \text { SSM } & \text { Stainer-Scholte liquid media } \\ \text { STING } & \text { Stimulator of interferon genes } \\ \text { Te } & \text { Expiratory time } \\ \text { Ti } & \text { Inspiratory time } \\ \text { Tr } & \text { Relaxation time } \\ \text { TVb } & \text { Tidal volume } \\ \text { vags } & \text { Virulence associated genes } \\ \text { vrgs } & \text { Virulence repressed genes } \\ \text { WBP } & \text { Whole-body plethysmography } \\ \text { wP } & \text { Whole-cell pertussis vaccines } \\ \text { WT } & \text { Wild-type }\end{array}$




\section{Chapter 1. Introduction}




\subsection{Bordetella pertussis overview}

Pertussis is a respiratory disease caused by the Gram-negative pathogen Bordetella pertussis $(B p)(1)$. Pertussis, also known as whooping cough, was originally reported during the 15th century in Persia (2). Patients with pertussis were often described as if they were being strangled without the capacity to expel the irritants in the lung causing the infection, simultaneously being incapable of inspiration (2). Paroxysmal coughing occurs so violently that blood can be expelled from the nose and mouth (2). It was not until 1906 that Jules Bordet and Octave Gengou were able to culture Bp in vitro (3). Bordet-Gengou (BG) agar was created as a defined medium for the isolation of $B p$, and it consists of glycerine, potato starch, blood, and agar (4). Potato starch is the critical component of BG, as fatty acids have been shown to inhibit $B p$ growth, and the starch works to sequester the fatty acids not only produced by $B p$, but from the sputum and nasal secretion of patients (3).

$B p$ is a respiratory pathogen that exclusively infects humans, particularly infants (5). Transmission of $B p$ occurs through the aerosolization of respiratory droplets containing $B p(6)$. Once inhaled, $B p$ adheres to the epithelial cells of the respiratory tract (7-9). In humans, incubation of $B p$ is on average is approximately $7-21$ days, and $B p$ infection can be broken down into three separate phases: catarrhal, paroxysmal, and convalescent (10). During the catarrhal phase of pertussis, mild symptoms ensue reflective of a common upper respiratory tract infection (11). Symptoms include rhinorrhea, sneezing, anorexia, and a cough that increases in severity and frequency as the infection progresses (11). One week following the first signs of illness, the patient then enters the paroxysmal phase, which is characterized by paroxysmal coughing lasting upward of one 
to four weeks (11). The Centers for Disease Control (CDC) defines paroxysms as multiple rapid, violent coughs followed by the classical whoop sound associated with pertussis, as a result of hurried and forced inspiration of air into the lungs (12). Along with the paroxysms; vomiting, exhaustion, uncontrolled mucus production, increase in white blood cell count (lymphocytosis and leukocytosis), bronchopneumonia, hypoxia, and choking can occur in infants (13). Four to six weeks following the onset of pertussis, the patient will enter the convalescent phase, where paroxysms begin to diminish; however, nonparoxysmal coughing can persist several months following infection (11). Macrolides are the standard treatment currently used to treat patients with pertussis. A fourteen-day round of erythromycin is recommended, azithromycin and clarithromycin have also been used (14). However, recently in China macrolide resistant $B p$ has emerged (15). In severe cases, pertussis can be fatal $(0.1 \%$ of pertussis cases from $2000-2016)$ especially in unvaccinated infants (16). It is estimated there are 24.1 million cases of pertussis worldwide contributing to about 160,700 deaths every year in children $<5$ years of age (17).

\subsection{Pathogenesis}

Animal models of pertussis have been instrumental in our limited understanding of $B p$ pathogenesis. As highlighted above, $B p$ is transmitted via the inhalation of respiratory droplets containing $B p$ from an infected host $(6,18)$. Upon entering the host, regulation of virulence factors is controlled via the master virulence regulator, bvgAS, which is a two-component signal transduction system (19-21). The sensor kinase bvgS is activated resulting in the phosphorylation of the response regulator, bvgA (22). Phosphorylation of bvgA and bvgS activation, commonly referred to as the bvg+ phase, leads to the 
expression of virulence associated genes (vags) such as pertussis toxin (PT), filamentous hemagglutinin (FHA), fimbriae (Fim), and adenylate cyclase toxin (ACT) as well as the repression of virulence repressed genes (vrgs) (22-26).

Upon inhalation, Bp uses adhesins such as FHA and Fim to attached to the ciliated epithelium of the respiratory tract (22). The autotransporter pertactin (PRN) has been suggested to play a role in initial attachment of $B p$, as PRN contains Arg-Gly-Asp motifs, which have been shown to play a role in epithelial binding (27). FHA is 232-kDa hairpinshaped protein that is expressed on the outer membrane of all Bordetella spp. Data from animal models have shown that FHA is crucial for $B p$ attachment and is highly immunogenic $(13,28)$. Proteomic analysis has suggested that FHA is one of the most abundant proteins of $B p(29)$. Bp lacking FHA have shown to have diminished capacity for colonization of the trachea $(30,31)$. Fim, which are filamentous in structure, are expressed on the cell surface and have been shown to be required for tracheal colonization (32). Fim negative strains have shown decreased capacity for bacterial attachment in vitro, and in vivo; additionally, Fim negative strains had reduced growth in the upper respiratory tract in mice $(32,33)$. Bp has 6 serotypes of Fim, with Fim1 being common amongst all Bp strains while Fim2 has been found on numerous Bp isolates (34).

Once $B p$ colonization occurs and infection is established, toxins are then released, the most notable of which is $\mathrm{PT}$. $\mathrm{PT}$ is an $\mathrm{AB}_{5} \mathrm{ADP}$-ribosylating toxin that is released and disseminated systemically (35). PT consist of 6 polypeptides from S1 to S5 subunits; S1 designates the enzymatically active A subunit of PT, while S2, S3, S4, and S5 comprise 
the B subunit (36-39). The S2-S5 subunits form a pentamer that binds to glycoproteins on eukaryotic cell membrane surfaces delivering the A subunit (36-39). In the cytoplasm, ADP-ribosylation of $G_{i}$ proteins occur rendering them inert $(40,41)$. This leads to an increase in cytosolic cAMP and dysregulation of $G_{i}$ protein signaling. The resulting effect leads to the dysregulation of innate immune cells particularly neutrophil and macrophage recruitment to the site of infection $(40,41)$. PT has also been shown to aid in adherence during colonization. Morse et al (1976) demonstrate that PT also causes leukocytosis during infection (42). Other studies have shown that PT can increase sensitivity to histamines, bradykinin, and serotonin (43).

ACT is also regulated by bvgAS and is secreted during infection. ACT is comprised of an adenylate cyclase (AC) domain and a repeat in toxin domain (RTX) linked with a hydrophobic domain responsible for pore formation (44-48). ACT remains localized, as most of the ACT remains associated with FHA on the bacterial surface, and secreted ACT quickly forms aggregates in solution rendering it inactive $(49,50)$. Upon secretion, ACT binds phagocytic cells via the $\alpha \mathrm{M} \beta 2$ integrin or CR3 receptor (51). Once bound, the AC domain is translocated into the cell where it can bind calmodulin to catalyze cAMP from ATP, resulting in the dysregulation of cellular signaling pathways (52). Most notably, cAMP formation results in the inhibition of antigen presentation by antigen presenting cells and induces apoptosis (53-55). ACT activity in macrophages results in membrane ruffling, cytoskeleton rearrangement, and loss of complement-mediated phagocytosis (56). 
Other virulence factors associated with $B p$ include dermonecrotic toxin, tracheal cytotoxin, and lipopolysaccharide (LPS) (57). Dermonecrotic toxin is a heat-labile toxin that causes necrotic lesions, and intravenous administration of dermonecrotic toxin is lethal in mice $(58,59)$. Tracheal cytotoxin is a disaccharide-tetrapeptide and during infection, tracheal cytotoxin leads to damage of the ciliated epithelial cells of the respiratory mucosa (57). LPS of $B p$ lacks the $O$ antigen and it is referred to as lipooligosaccharide (LOS), which is extremely immunogenic; and $\mathrm{O}$ antigen negative mutants are attenuated during infection (22). 


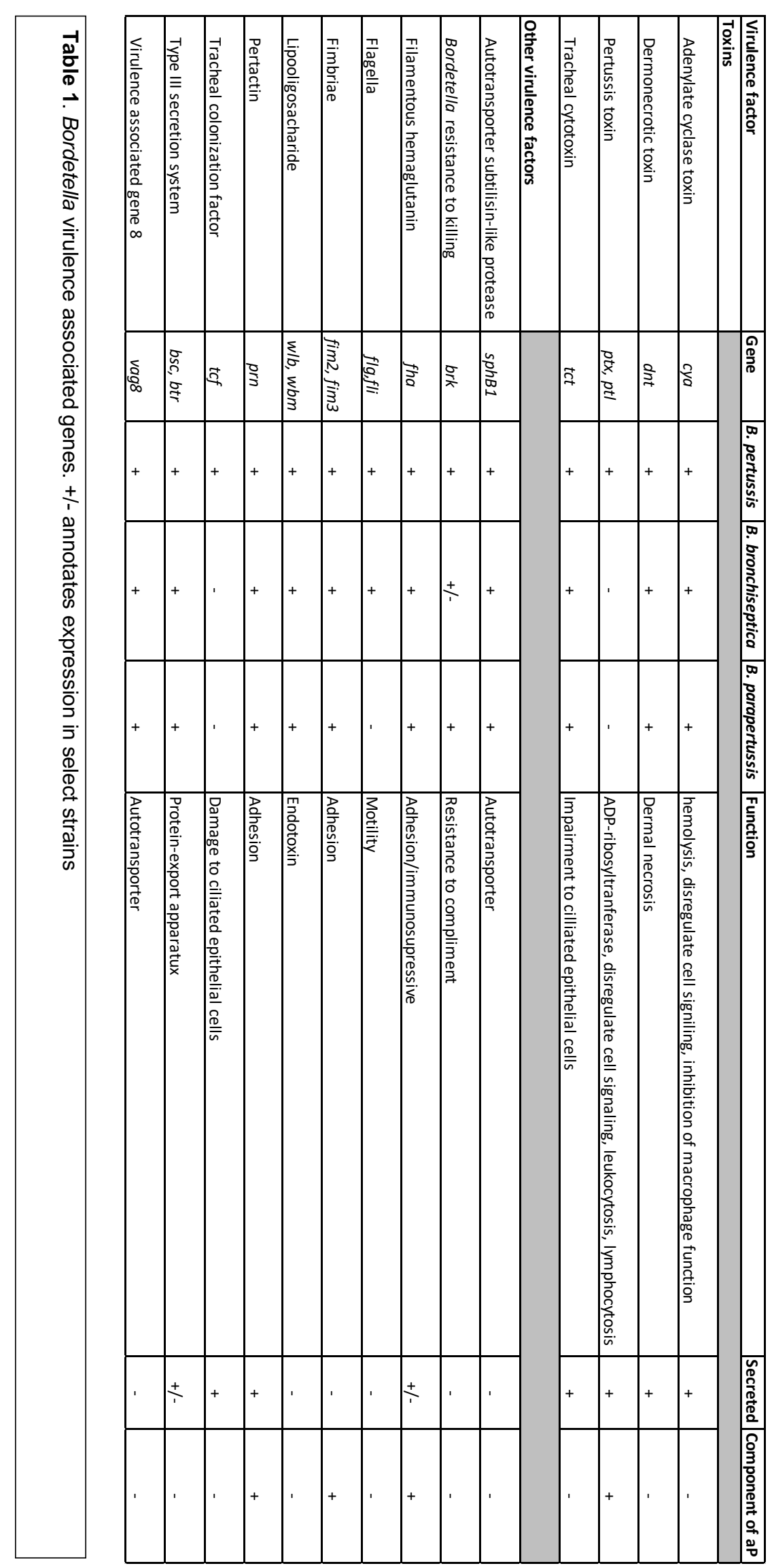




\subsection{Pertussis vaccines}

In the pre-vaccine, era the number of pertussis cases ranged from $150,000-200,000$ cases in the United States annually (Fig. 1) (59). After the in vitro culture of $B p$, the development of whole-cell pertussis vaccines (wP) immediately ensued. The first licensed wP was made from aliquots of killed $B p$ in 1914 (60). It was not until 1948 that wP was combined with diphtheria and tetanus toxoids creating the Diphtheria Tetanus Pertussis vaccine (DTP) $(11,61,62)$. Widespread use of DTP in the United States led to a $90 \%$ decrease in the number of reported $B p$ infections (63). After implementation of the DTP, the number of pertussis cases in the Unites States was $<1,000-5,000$ cases between 1970-1980 (Fig. 1) (59). With the decrease in number of pertussis cases, it was hypothesized the disease was eradicated (64). Despite the efficacy of this vaccine, serious adverse side-effects ensued including swelling and redness at the injection site, fever, and uncontrollable crying of infants (65). In rare instances, vaccine-induced encephalopathy was reported following DTP immunization (66). Due to these side-effects, patient compliance regarding DTP vaccination decreased, particularly in Britain, Japan, and United States (11). After two infant mortalities were reported following DTP immunization, the Japanese Ministry of Health and Welfare formed the Pertussis Vaccine Study Group for the development of a safer and effective acellular pertussis vaccine (aP, DTaP, Tdap) in 1981 (67). Low to middle income countries still use DTP vaccines today to protect against pertussis. 
It was not until 1984 that Sato and Sato developed the first aP in Japan, which consisted of formaldehyde treated FHA and formalin treated PT (67). Vaccination with the newly developed aP was protective in children over the age of 2 years old (68). A subsequent

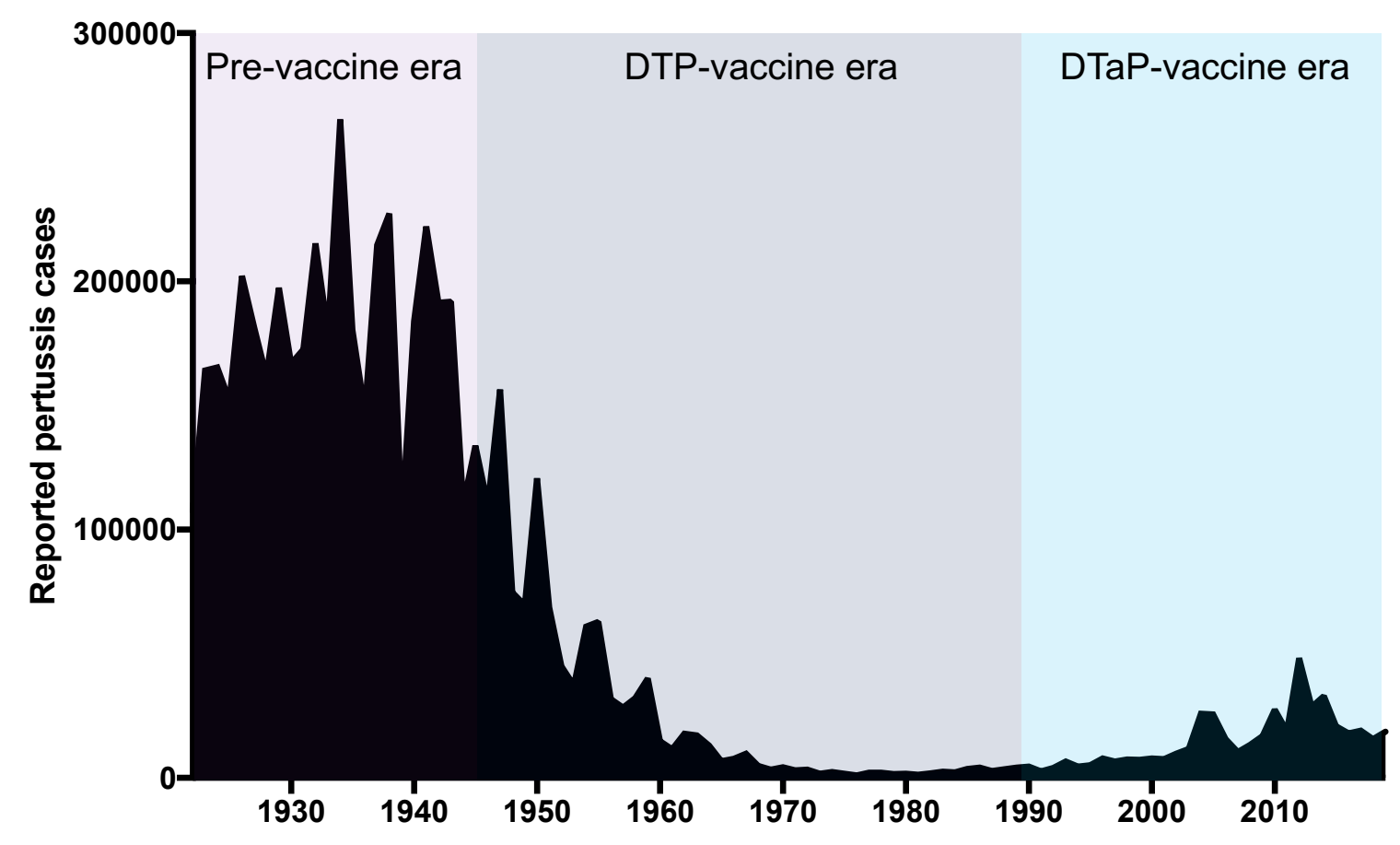

Figure 1: Reported pertussis incidence in the United States from 1922-2019. Adapted from the CDC.

vaccination study also confirmed that the aP was less reactogenic than the $\mathrm{wP}$, despite being protective and generating a serological response to the vaccine antigens (69). During the development of the aP, 13 antigens were originally selected for testing (70). The antigens that were selected to be included in the aPs were FHA, Fim, PT, and PRN, as each one of these antigens were immunogenic, providing a serological response after vaccination and playing a role in Bp pathogenesis (70). ACT was thoughtfully considered to be included, but with the limited research surrounding ACT at the time and the limited availability of a purified product, ACT was left out of aP formulations. It was not until 1996 
that DTaP was licensed for use in infants in the United States. One year later, the Advisory Committee on Immunization Practices (ACIP) recommended DTaP vaccination over DTP vaccination in infants. It was not until 2006, that the ACIP recommended that a Tdap booster vaccine be administered during adolescence.

\subsection{Waning efficacy of the current acellular vaccine}

After the switch from the DTP to DTaP, there has been a significant increase in the number of pertussis cases, especially in the United States (71) (Fig. 1). While nationwide vaccine coverage is $95 \%$ in the United States, the incidence of pertussis has risen in the past 10 years as the number of only DTaP vaccinated individuals has increased in size. (72). Studies suggest that four years after a patient's last DTaP vaccination, the induced vaccine efficacy is only nine percent, which is unacceptable vaccine protection (5). While vaccine coverage remains high, the population dynamics are changing, and more people are DTaP immunized as new generations are born and the DTP immunized only generations age. Numerous studies have demonstrated waning efficacy following DTaP vaccination in parallel with the emergence of genetically divergent strains of $B p$ (73-79). Increased surveillance of $B p$ has led to the identification of clinical isolates that do not express PRN, FIM, and even PT, the hallmark toxin of the organism (80). Research has shown that PRN mutant strains have been present since the 1990 s, while only $15 \%$ of isolates express PRN currently (81). Bp is evolving to not express the proteins that are found in the current aP (80). One plausible hypothesis to explain these observations is that circulating $B p$ strains have evolved due to aP vaccine pressure. Vaccine-driven evolution of $B p$ could contribute to the waning efficacy of the aP. While we know the current strains are genetically different, we do not know if this genetic variability affects 
virulence, disease burden, toxicity, or fitness of the pathogen (79). With that said, it is imperative to find a means to increase the efficacy of the current pertussis vaccines.

\subsection{Recent Evolution of Bp}

Since the introduction of DTaP in 1996, the Bordetella research community has observed cyclic increases in the reported number of pertussis cases caused by the bacterium $B p$ (82). Current surveillance of $B p$ in the United States has identified genetic changes alongside the reemergence of pertussis $(83,84)$. This is highly alarming as $B p$ is naturally considered to be a monomorphic pathogen (85). Recent clinical isolates have been identified that are deficient in either FHA, PRN, and PT, which are components of the aP, despite adequate vaccine coverage (86-89). New data generated by the CDC highlights the genetic divergence of current circulating strains of pertussis from the reference strains that originally helped to produce the vaccine (83). The clinical isolates differ due the presence of SNPs and chromosomal rearrangements which could contribute to the pathogen's overall fitness and ability to infect. It is essential to critically evaluate the significance of the evolution of these strains and their potential disease burden.

\subsection{Animal models of pertussis}

$B p$ is exclusively a human respiratory pathogen, and as a result, a diverse repertoire of animal models have been used. The evolution of animal models of pertussis has been fundamental in our understanding of $B p$ pathogenesis and the formulation of vaccines against this pathogen. Robust efforts have been made to develop animal models capable of recapitulating pertussis as seen in humans. Currently, mice have been widely accepted and are used to study pertussis, in addition to non-human primates, piglets, and rats. 
Cost, accessibility of reagents, and genetic manipulation also contribute to the feasibility of mice as a model of pertussis.

Mice infected with $B p$ develop leukocytosis, hyperinsulinemia, histamine sensitization, and comparable respiratory infection to humans (90). In mice, three different routes of $B p$ challenge have been explored: intracerebral (IC), intranasal (IN), and through aerosol exposure. These routes of $B p$ challenge were selected as $B p$ can only grow in the respiratory tract or the brain of mice (11). In 1947, Pearl Kendrick and Grace Eldering developed the IC mouse model to assess WP vaccine efficacy, as the pertussis community at the time needed a way to test pertussis vaccine potency (91). IC $B p$ challenge in mice was considered the gold standard for testing wP vaccine potency at the time, as results correlated to similar vaccine potency tests in field trials by the Medical Research Council (92). In short, mice were intraperitoneally (IP) vaccinated with wP, followed by a subsequent $10^{5}$ CFU IC Bp challenge at 14 days post-vaccination (91). Vaccine efficacy was determined by the survival of the mouse (91). After development of the $\mathrm{aP}, \mathrm{IC}$ challenge model in mice failed to predict aP vaccine efficacy; however, the IN $B p$ challenge model was successful at evaluating aP vaccine efficacy (93). Vaccine efficacy was then determined not solely on survival of the mouse, but also the serological response to vaccine antigens and bacterial clearance in the respiratory tract (93). Aerosol challenge of $B p$ was investigated as IN $B p$ challenges rely on depositing large bacterial loads into the lungs of mice rather than uniform bacterial placement in the respiratory tract (22). Aerosol challenge resulted in a more uniform respiratory tract infection compared to IN $B p$ challenge (94). Intraperitoneal challenge of $B p$ in mice was also investigated as a 
potential method for challenge; however, $B p$ did not readily colonize in the peritoneal cavity (95). Limitations of the mouse model of pertussis include the absence of a paroxysmal cough, reproducible pertussis pneumonia, transmission, and limited nasal colonization (22).

Piglets have also been used as an animal model to study pertussis (96-99). In 2005, Elahi et al demonstrated that 4-week-old piglets can be infected from intrapulmonary inoculation of $B p(96)$. Bp challenged piglets display nonparoxysmal coughs, labored breathing, nasal discharge, and fever (96). Following challenge, all piglets developed bronchopneumonia, and histology confirmed pulmonary inflammation of neutrophils and macrophages in the airway (96). One caveat of the pig model of pertussis is that pigs older than five weeks of age are not readily infected following $B p$ challenge (97). This phenomenon is observed as the peptide beta-defensin 1 is present in the upper respiratory tract after five weeks of age and protects against $B p$ challenge (98). The pig model of pertussis has demonstrated that maternal immunization confers protection to offspring following vaccination (99). Pregnant sows were vaccinated with heat inactivated $B p$ and their newborn piglets fed from their mothers for approximately 5 days followed by subsequent $B p$ challenge (99). Newborn piglets were protected against marked pathological changes and bacterial burden in the respiratory tract (99).

Besides rats, nonhuman primates are the only other known model of pertussis that share similar coughing manifestation following $B p$ challenge $(100,101)$. IN inoculation of $B p$ in nonhuman primates results in lymphocytosis, leukocytosis, and transmission of $B p$ (101- 
106). Recently, Warfel et al (2012) evaluated baboons as a potential pertussis animal model. Olive baboons were selected as they have similar body temperatures to humans (101). Baboons IN challenged with $10^{10} \mathrm{CFU}$ of $\mathrm{Bp}$ resulted in cough, bacterial colonization, and leukocytosis (101). Warfel et al (2014) also showed that aP vaccinated baboons were protected against pertussis disease following subsequent $B p$ challenge; however, they were not protected against bacterial colonization and transmitted $B p$ to unvaccinated baboons (107). WP immunized and convalescent baboons rapidly cleared the infection following $B p$ challenge (107). Protection afforded by the aP was suggested to be a more Th2-skewed immune response, while wP and convalescent immunity induces a mix of Th1 and Th17 immune responses (107-109). These data illustrate that the immune response generated by aP vaccination protects against pertussis disease but fails at protecting against $B p$ transmission to an unvaccinated host; however, the Th1 and Th17 immune response generated by wP and prior infection prevents colonization and the subsequent transmission of $B p(107)$. More recently, rhesus macaques have been used as a nonhuman primate model of pertussis $(110)$. While the nonhuman primate model of pertussis shares many clinical manifestations of pertussis similar to humans, the expensive animal cost, limited and specialized facilities, and ease of use limit the use and availability of this model.

\subsection{Coughing rat model of pertussis}

The coughing rat model of pertussis was first introduced in 1939, and nine studies in total have been published using the coughing rat model of pertussis to study $B p$ pathogenesis

and vaccine-mediated immunity (Table 1). Two separate $B p$ challenge methods have been used in the development of the coughing rat model of pertussis. In 1939, Hornibrook 
and Ashburn IN challenged rats with $B p$, as mice were readily infected following IN challenge of $B p$ (111). IB inoculation of $B p$ was not utilized until 1989, which was developed in part as IB inoculation of Pseudomonas aeruginosa resulted in an respiratory infection (112). During this time, Hornibrook was also working on developing a semisynthetic liquid medium for the isolation of soluble antigens (113). 


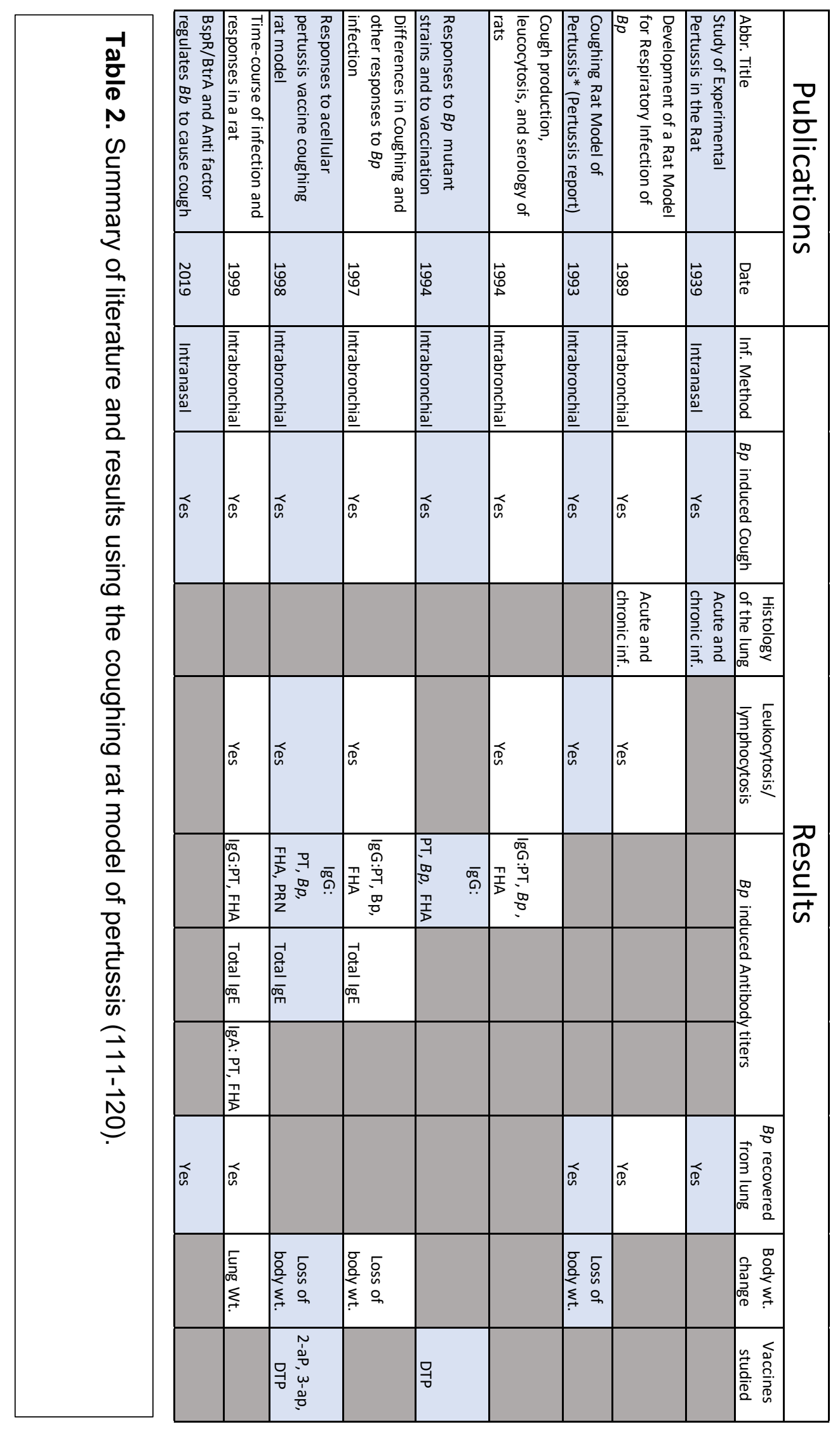




\subsubsection{Intranasal challenge}

It was first reported in 1939 that young rats developed paroxysmal cough after IN inoculation of $B p$ (111). Hornibrook and Ashburn hypothesized that rats could be a suitable animal model to study pertussis, as mice infected with $B p$ produced pneumonia and death following IN challenge (111). To test this hypothesis, 3-week old rats were intranasally infected with $B p$ and survival was monitored for up to 10 days post-challenge (111). Following challenge, approximately 12 percent of $B p$ infected rats died after two days post-challenge (Fig. 2) (111). By day 9 post-challenge, 72.5 percent of $B p$ infected rats died (Fig. 2) (111). Coughing was recorded for 12 of the $51 \mathrm{Bp}$ infected rats, and it was noted that on average, coughing did not appear until approximately 8 days postchallenge (111). Coughing continued in some rats for up to 60 days post-challenge (111).

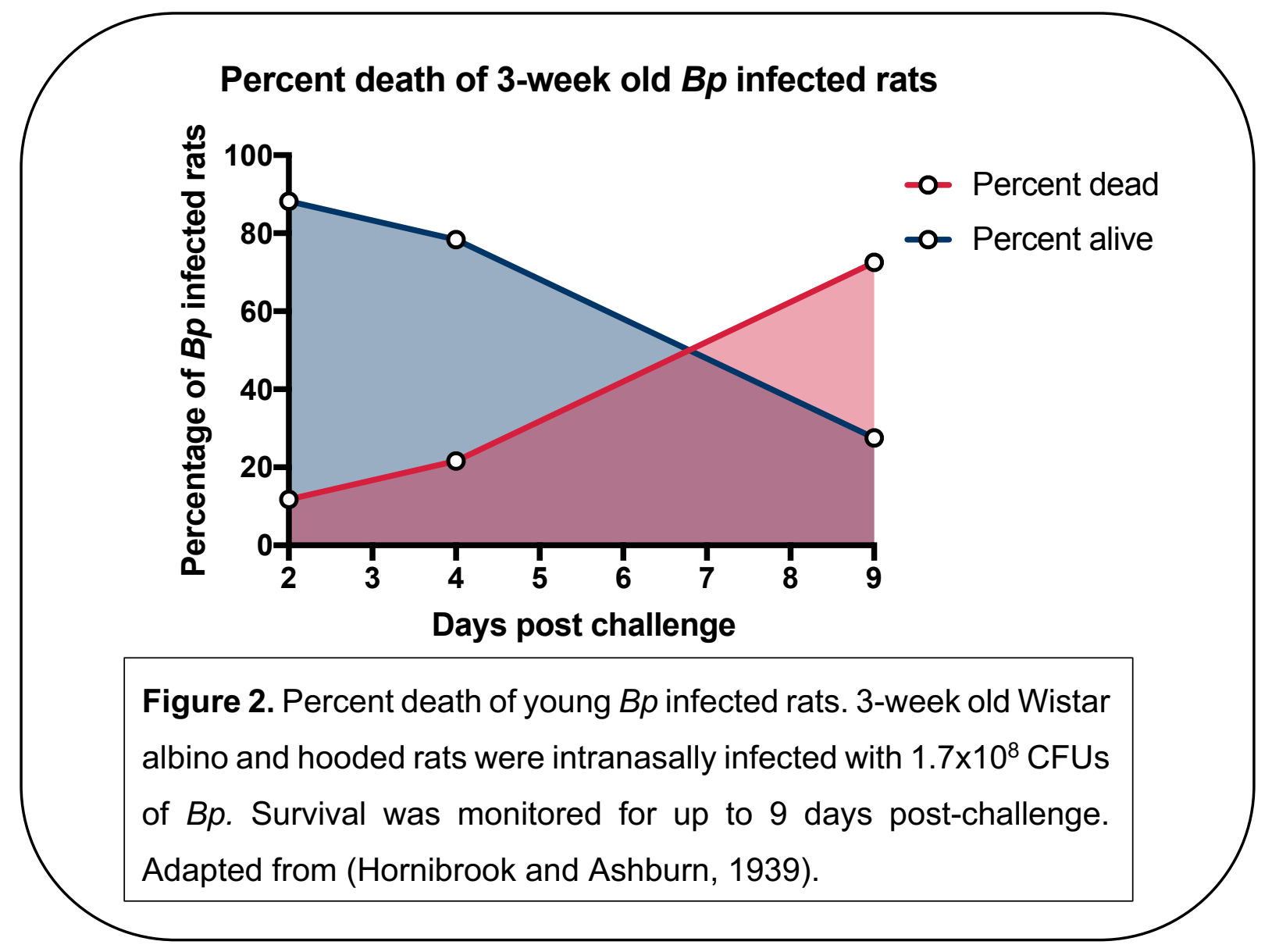


Pathological examination revealed that $B p$ colonization of the lung resulted in interstitial bronchopneumonia combined with exudate infiltration into the alveoli (111). By day 3 post-challenge, infiltrating neutrophils accumulated in the lung, followed by monocyte infiltration by day 6 post-challenge (111). It was not until 2019, that IN inoculation of Bordetella bronchiceptica $(B b)$ was used to evaluate mechanisms responsible for $B b$ elicited cough (114). In summary, these data revealed that rats can be readily infected with $B p$ and infected rats developed cough-like paroxysms similar to humans.

\subsubsection{Intrabronchial challenge}

To the best of our knowledge, the rat model of pertussis was not used again for almost 50 years. It can be hypothesized that the lack of studies using the coughing rat model of pertussis was due to the development and implementation and success of DTP (112). As safety concerns surrounding the DTP emerged, so too did the need for an adequate animal model to understand the pathogenesis of $B p$. Significant progress was made, in parallel, utilizing mice as a challenge model to identify protective aP antigens; however, mice lacked several aspects of disease as seen in humans: Most notably, $B p$ induced cough combined with an upper respiratory tract infection and a repeatable pertussis elicited pneumonia. Woods et al (1989) decided to utilize IB challenge Sprague-Dawley rats with $B p$ encased in agar beads, as this challenge method was successful at establishing Pseudomonas aeruginosa infection in rats (112). In brief, $10^{7} \mathrm{CFUs}$ of $B p$ was added to a mixture of melted $2 \%$ agar and heated mineral oil, and rapidly cooled creating Bp encased agar beads (112). Two hundred adult ( 200 gram) Sprague-Dawley rats were challenged with either Tohama I (virulent) or Tohama III (avirulent) (112). Tohama I was recovered from the lung for up to 7 days post-challenge, while no infection 
was observed in Tohama III infected rats (112). No morbidity was observed following challenge (112). Pathological analysis revealed lymphocyte infiltration into the bronchi at day 3 post-challenge, followed by inflammation of the mucous membranes and bronchioles by monocytes and polymorphonuclear cells (PMNs) by days 7 and 21 postchallenge (112). It was also noted that intrabronchial challenge of $B p$ induced a "paroxysmal like" cough as seen in humans (112). A following study performed by Wardlaw et al (1993) utilized sound activated tape recorders to detect cough for up to 21 days post challenge (115). Isolated coughs along with multi-cough events, similar to a paroxysmal cough, were detected (115). Peak numbers of cough were recorded at day 10 post-challenge and an increase in white blood cell counts were also observed over the 21-day infection (115). Hall et al (1994) further confirmed that Bp infected rats developed paroxysmal cough and leukocytosis (116). The serological response to $B p$ challenge was also investigated: Intrabronchial challenged rats developed IgG antibody titers to $B p$ sonicate, PT, and FHA at day 28 post-challenge (116). It was not until 1999, that Hall et al illustrated the unique inverse relationship between bacterial burden and cough (117). By day 10 post-challenge, as cough counts were the highest, bacterial burden in the lung was the lowest (117).

With the development of a pertussis model that shared similar coughing manifestations as humans', mechanisms responsible for the $B p$ induced cough were explored. Rats challenged with Phase 1 (virulent) strains of Bp such as 18-323, Tohama I, and L-84 induced coughing at days 9-14 post-challenge (118). Phase 4 (avirulent) strain L-84 induced no coughing, highlighting that virulence factors are responsible for $B p$ induced 
cough (118). Bp strain 357 harboring a transposon-insertion in the region encoding for PT induced no coughing following challenge (118). Similar results were observed in rats challenged with Bordetella parapertussis, which does not produce PT (118). Although debated, these results suggest that PT plays a role in the induction of cough during pertussis infection. Further studies are needed to accurately depict the factor(s) inducing cough following infection.

Strains of rats were also evaluated in the development of the rat model of pertussis. While past studies relied on Sprague-Dawley rats to evaluate Bp pathogenesis, Brown Norway, Lewis, and Hooded Lister rats were also tested (119). Brown Norway, Lewis, Hooded Lister, and Sprague-Dawley rats all coughed following IB challenge of $B p$, although Sprague-Dawley rats coughed more consistently compared to the other strains (119). Leukocytosis was observed in all four strains of rats, as high as 4-fold compared to the non-infected controls (119). Younger, 4-week-old rats had an increased propensity toward infection as a decrease in weight gain and increase in mortality were observed compared to 6-week-old rats, regardless of the rat strain (119). Hall et al (1997) further illustrated that total IgE in the serum of Sprague-Dawley, Brown Norway, Lewis, and Hooded Lister rats were elevated following $B p$ challenge (119). Anti-Bp and anti-FHA IgG antibodies were detected in all rat strains; however, Hooded Lister and Lewis rats did not develop anti-PT IgG antibody titers post challenge, suggesting host genetics could possibly play a role (119). 
Limited studies have investigated the vaccine efficacy of DTP and DTaP in the coughing rat model of pertussis. To measure vaccine efficacy of the DTP, one single human does was administered IP to 3-week-old Sprague-Dawley rats, followed by subsequent intrabronchial $B p$ challenge 3 weeks later (118). DTP vaccination provided protection against $B p$ elicited cough compared to mock vaccinated challenged rats (118). DTP vaccinated rats also developed serum anti-FHA and anti-Bp antibody titers at day 28 post challenge (118). Hall et al (1998) also confirmed that two 1.5 human doses of Evans' DTP administered subcutaneously at 21 and 7 days before challenge protected against cough upon intrabronchial Bp challenge (120). SmithKline Beecham's 3-component aP vaccine containing detoxified PT (PTd), FHA, and a 69kDa antigen (presumably pertactin (PRN)) as well as Connaught;s 5-component containing PTd, FHA, agglutinins 2+3 (fimbriae), and PRN administered subcutaneously 21 and 7 days before challenge were also protective against $B p$ induced cough (120). Both aP vaccines and Evans' DTP protected against $B p$ induced leukocytosis (120). Vaccination with PT, FHA, PRN, and fimbriae alone with no adjuvant did not significantly protect against cough compared to mock vaccinated challenged rats (120). These data suggest that vaccination against pertussis needs to be multi-valent and adjuvanted. Overall, aP and wP vaccines were protective against $B p$ induced cough and leukocytosis (120). Protection against bacterial burden in the coughing rat model of pertussis following vaccination has yet to be established.

In summary, the coughing rat model of pertussis can be used as a tool to not only evaluate $B p$ pathogenesis, but also as a preclinical model to investigate vaccine efficacy. The coughing rat model of pertussis can be utilized to investigate mechanisms responsible for 
coughing during $B p$ infection, as well as in the validation of new vaccine formulations or routes of immunization to determine their ability to protect against $B p$.

\subsection{Natural immunity against $B p$}

As described above, $B p$ is a respiratory pathogen. During infection, $B p$ colonization remains strictly within respiratory tract, and dissemination is rarely observed during pertussis infection $(13,121)$. Both the innate and adaptive immune responses are vital to recognition and defense against $B p$. Only in mice with immunodeficiencies does $B p$ disseminate to other organs such as the spleen and liver (122). Recognition of $B p$ in the airway induces the infiltration of macrophages, dendritic cells, neutrophils, and NK cells (123-125).

Macrophages have been shown to play a role in immunity against $\mathrm{Bp}$. In mice, depletion of macrophages by liposome-encapsulated clodronate in the airway led to an increase in bacterial burden in the respiratory tract up to 2 weeks post-challenge (126). Macrophage killing of $B p$ by nitric oxide has been shown to be increased with supplementation of IFN$\gamma(127,128) . B p$ challenge in IFN- $\gamma-/-$ mice results in dissemination of $B p$ and mortality (122, 129). IFN- $\gamma$ secreting Th1 cells were associated with recovery in children with pertussis $(130,131)$. Altogether, these data show that macrophages are essential in clearance and protection against $B p$.

The role of neutrophils in pertussis immunity has been quite a contrast to that of macrophages. Studies in mice have shown that infiltration of neutrophils occurs as early as 5 days post-challenge and as late as 14 days post-challenge $(132,133)$. During naïve 
infection, neutrophils seem to not play a role in the clearance of $B p$, presumptuously, due to the inhibitory function of both PT and ACT $(41,134)$. PT works by delaying the overall neutrophil response during the initial stages of infection, and ACT works by dysregulating neutrophil phagocytic propensity $(41,135)$. Neutrophils of convalescent mice or mice receiving sera with opsonizing antibodies toward $B p$ are capable of antibody-mediated phagocytosis; suggesting they play a role in $B p$ clearance in an immune competent host (41). Neutrophil extracellular traps (NETs) have also been observed during Bp infection (136).

Induction of adaptive immunity towards $B p$ is necessary for protection against pertussis. Both mice and patients previously infected with $B p$ have measurable amounts of IgA in their nasal fluids, and Bp specific IgG antibodies in their serum (137). In humans, passive immunization of $B p$ specific sera resulted in a decrease in overall disease severity (138). Patients with an increase in anti-PT and anti-PRN antibody titers had a lower incidence in pertussis than members of the same house-hold after $B p$ exposure (139). In mice, the generation of antibodies following $B p$ challenge does not result in significant levels of antibody titers until approximately 1 month post infection, suggesting antibody responses are not a major player in immunity to a primary infection (137). Immunization with $B p$ specific antigens results in the induction of antibody titers to $B p$, and passive immunization results in protection against pertussis (140). $\mathrm{lg}$-/- mice fail to clear pertussis infection, eventually developing a chronic respiratory infection; however, the transfer of $\mathrm{Bp}$ immune competent mature $\mathrm{B}$ cells was capable of restoring protection against pertussis $(140,141)$. 
Cell meditated immune responses have also been shown to play a role in immunity to $B p$. CD4+ T cells specific for PT, FHA, and PRN were isolated from infants following pertussis infection. The isolated T cells were found to secrete IFN- $\gamma$, with limited secretion of IL-4 and IL-5, demonstrating that $B p$ infection induces a Th1 rather than Th2 immune response $(130,131)$. Athymic nu/nu mice challenged with $B p$ resulted in chronic infection and even death; however, this phenotype was rescued by adoptive transfer of splenic CD4+ cells but not CD8+ cells (137). CD4+ positive mice isolated from Bp infected mice secrete Th1/Th17 associated cytokines: IFN- $\gamma$ and IL-17 $(122,129,142)$. IFN- $\gamma-/-$ and IL-17 -/- mice challenge with $B p$ result in an increase in disease severity and bacterial colonization (142). Additional studies in the nonhuman primate model of pertussis illustrate that convalescent baboons develop protective Th1 and Th17 immune responses (108). Taken together, these data conclude that $B p$ infection induces a protective Th1/Th17 immune responses.

In summary, protection against pertussis requires both innate and adaptive immune responses (Fig. 3). Natural infection induces protective Th1/Th17 T cell responses as well as protective humoral responses. As a result, innate immune cells recruited to the site of infection can become activated and opsonization of Bp results in antibodymediated phagocytosis. 


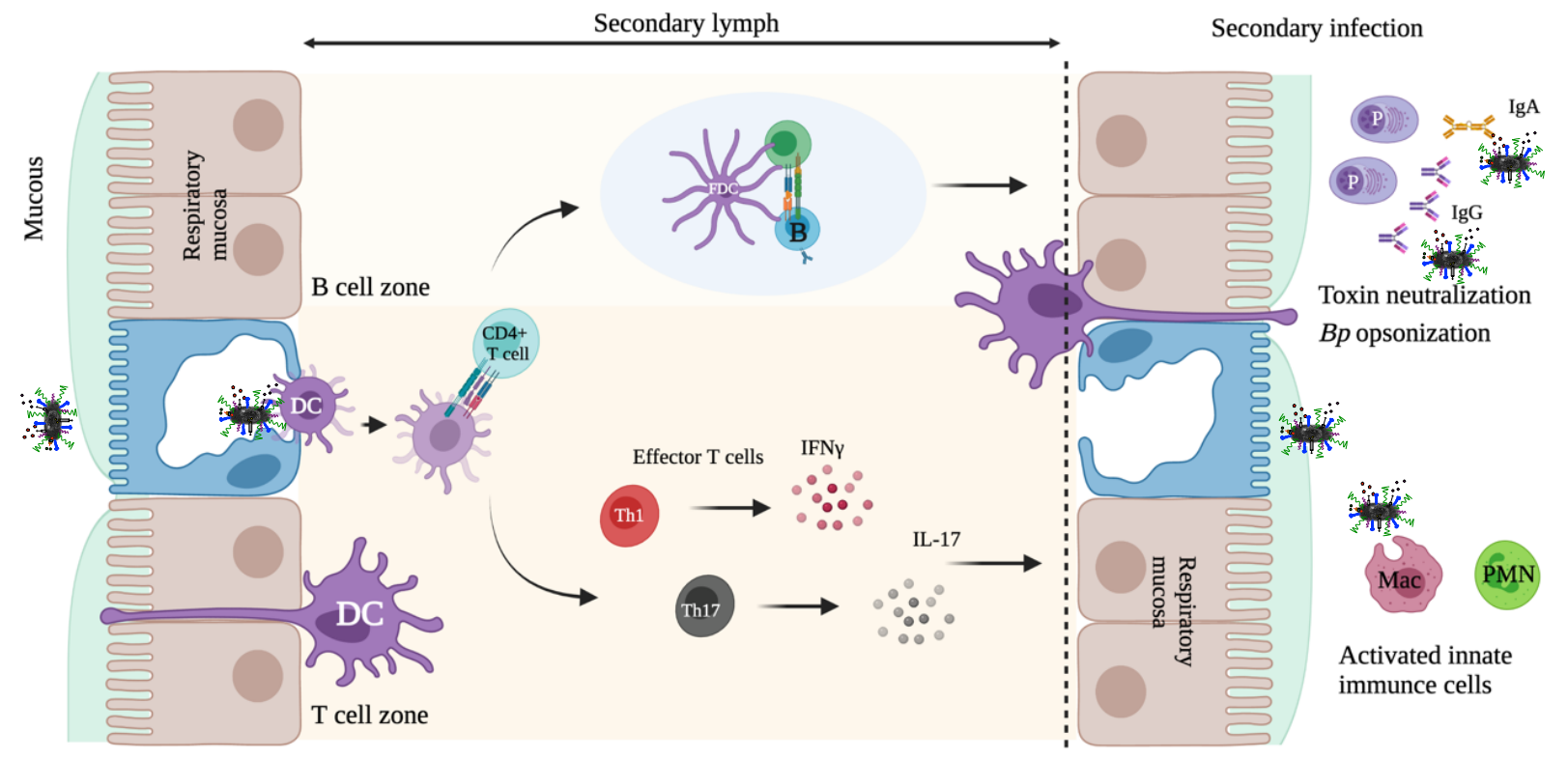

Figure 3. Summary of the immunity generated from natural infection. Natural $B p$ infection induces both a humoral and effector Th1/Th17 immune response. Adapted from (Kapil and Merkel, 2019). Created with Biorender.com

\subsection{Vaccine mediated immunity}

After the resurgence of pertussis, considerable efforts have been made to dissect the different immune responses induced from either wP or aP vaccination. Recent work in humans has shown that although DTaP vaccination induces higher IgG antibody titers compared to DTP vaccinated individuals, vaccine-mediated protection from DTP immunization lasts considerably longer $(143,144)$. Further evidence supporting variation in vaccine-mediated immune response was identified when characterizing the $T$ cell responses following DTaP and DTP vaccination. Patients receiving DTP vaccination resulted in a robust Th1 phenotype, while DTaP vaccinated patients presented with a Th2 immune response $(145,146)$. Studies in mice illustrate that DTP vaccination resembles similar immune responses to natural infection. DTP immunization induces a robust pro 
inflammatory response, further activating neutrophils' and macrophages' ability to clear $B p$ from the airway $(142,147,148)$. Mice receiving DTP vaccination cleared $B p$ infection more readily compared to DTP immunized mice deficient in IFN- $\gamma$ and IL-17, suggesting that vaccine-mediated immunity induced by DTP is driven by IFN- $\gamma$ and IL-17 $(140,142)$. Vaccination with DTaP in mice results in a predominant Th2 immune response but also a Th17 response, likely due to the adjuvant effect of alum. IL-4 -/- mice vaccinated with DTaP had similar protection to wild-type (WT) mice also vaccinated with DTaP and the observed protection was mediated by IL-17 (1). In humans, IL-17 mediated protection induced from DTaP vaccination is absent $(142,149,150)$. The antibody responses following DTP and DTaP vaccination are also different. DTP consists of a diverse repertoire of antigens, while DTaP consists specifically of 3-5 antigens: PT, FHA, PRN, Fim2, and Fim3. Primarily the antibody type generated from DTP immunization is IgG2a while DTaP vaccination results in IgG1 antibodies $(1,142,149)$. Warfel et al (2014) illustrated that DTaP vaccinated baboons were protected from pertussis; however, they were not protected against $B p$ colonization (149). Furthermore, DTaP vaccinated baboons were capable of transmitting $B p$ to unvaccinated baboons (149). DTP vaccinated baboons were protected against $B p$ burden in the airway (149). These data demonstrate the clear differences in the generated vaccine-mediated immune responses (Fig. 4). Taken together with our understanding of convalescent immunity, these data can be used as a roadmap to an improved DTaP vaccine. 

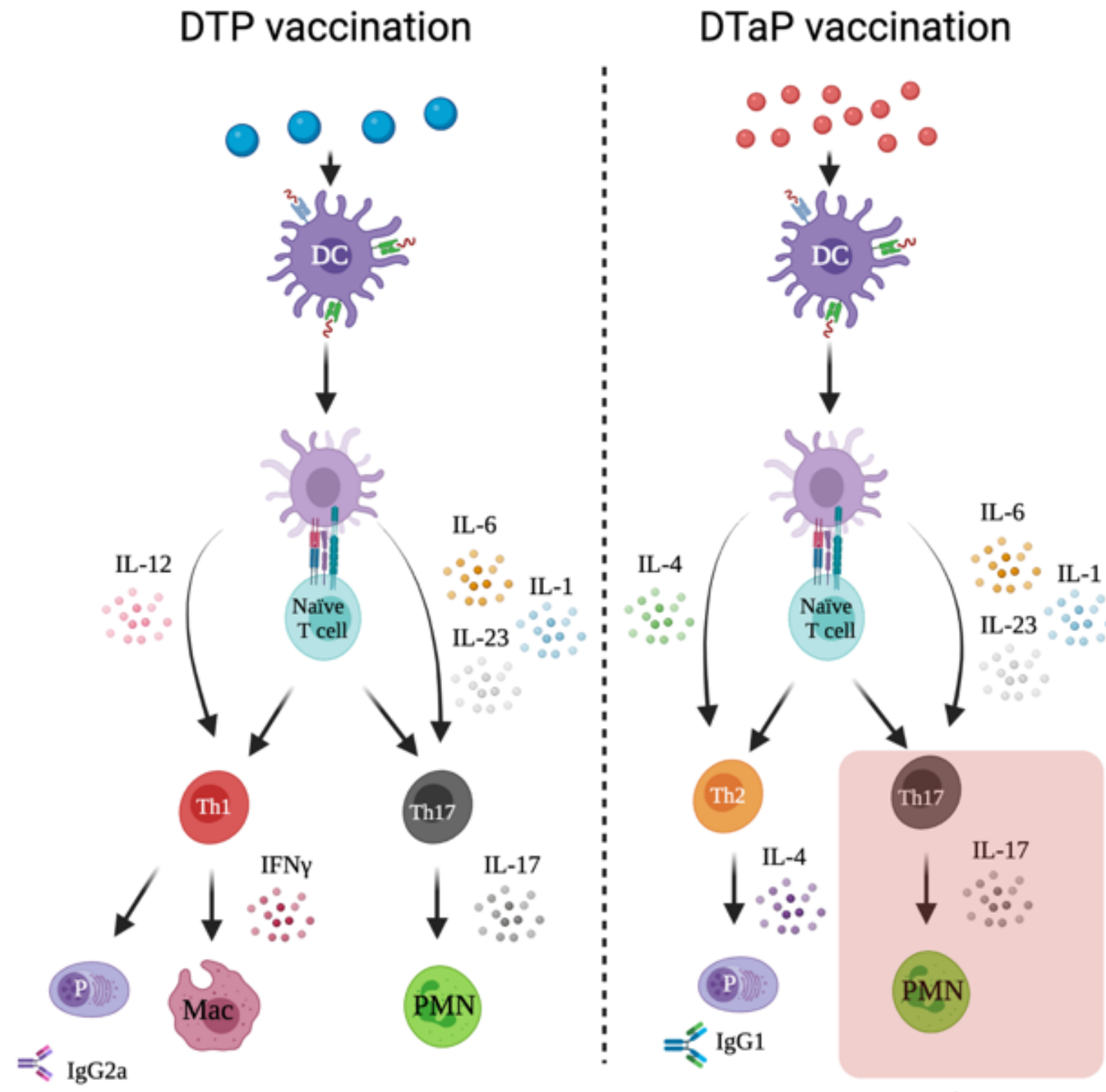

protective

response in mice

Figure 4. Summary of the DTP and DTaP vaccine mediated immunity. DTP immunization induces both a Th1/Th17 immune response similarly to natural infection, while DTaP immunization induces more of a Th2 driven immune response. Adapted from (Higgs, 2012). Created with Biorender.com 


\subsection{Mucosal immunization of DTaP}

With $B p$ strictly being a respiratory pathogen, stimulating local immunity at the respiratory mucosa is critical for the defense against pertussis. Humans previously infected with $B p$ develop longer lasting immunity (approximately up to 20 years) (151). On average, the protective immune response generated from DTaP vaccination is approximately 4-12 years (151). In the nonhuman primate model, convalescent baboons cleared Bp from the respiratory tract following challenge, while $\mathrm{DTaP}$ vaccinated baboons failed to protect against $B p$ colonization (149). DTaP immunization induced protection against pertussis disease, but not bacterial burden, allowing for asymptomatic transmission of $B p$ to naïve baboons (149). These data suggest that mucosal immunity is necessary for generating longer lasting protection against $B p$ but also in protecting against asymptomatic transmission of $B p$ to a naïve host.

Both intranasal (IN) and oral gavage (OG) immunizations have been explored to stimulate a protective mucosal immune response. In mice, IN administration of DTaP has resulted in protection against bacterial burden in the respiratory tract $(152,153)$. As a result, both anti-Bp and anti-PT IgG antibody titers were induced following IN DTaP vaccination in the serum, in addition to IgA antibody titers specific to $B p$ in the lung $(152,153)$. Wolf et al (2021) illustrated that the humoral responses elicited from IN DTaP immunization remained up to 6 months post-vaccination and were protective against bacterial burden post challenge (153). BPZE1, which is a live attenuated vaccine strain of $B p$, has also been administered IN and has shown to be protective in mice (154). In clinical trials, IN vaccination of BPZE1 elicits both systemic and mucosal antibody titers specific to $B p$ (155). In infants, oral vaccination of wP resulted in the production of mucosal antibody 
titers in the saliva, as well as systemic Bp specific antibody titers in their sera (156). In a following study, the frequency of pertussis was lower in newborns for up to one year following oral vaccination of $10^{12} \mathrm{CFUs}$ of killed $\mathrm{Bp}(157)$. Oral vaccination resulted in both IgG antibody titers in the serum and IgA titers in the saliva. Studies in mice have shown that oral vaccination of bacterial vectors (Salmonella typhimurium and Escherichia coli) expressing FHA elicited anti-FHA titers in the lung (158). These data suggest that mucosal vaccination can stimulate a protective immune response at the respiratory mucosa.

\subsection{Overall Objective}

The objective of this body of work is to build upon the already established coughing rat model of pertussis and use this model as both a tool and preclinical model to investigate the efficacy of mucosal immunization with DTaP. As highlighted above, with the implementation of DTaP vaccines we have seen a resurgence of pertussis, especially in the United States. Improved animal models combined with improved vaccination strategies are a necessity to combat pertussis. In chapter 2 , the overarching goal was to further develop the rat model of pertussis that recapitulates the same clinical manifestations of pertussis in humans. We intranasally challenged rats with reference strain Tohama 1, which was isolated in 1954 and is one of the most commonly used lab strains, and strain D420 which is a current representative clinical isolate that was isolated in $2002(159,160)$. To count coughs and the respiratory profile following challenge, we utilized whole body plethysmography (WBP). WBP revealed that challenge with D420 resulted in an increase in cough over the twelve-day infection compared to Tohama 1 infected rats. In chapter 3 , we utilized the rat model of pertussis to evaluate IN and OG 
immunization of DTaP as a potential mucosal vaccination strategy compared to IM administration of DTP and DTaP. Our results show that mucosal vaccination of DTaP through oral and intranasal immunization protects against both bacterial burden and $B p$ induced cough. Mucosal vaccination also induced both systemic ( $\lg G)$ and mucosal $(\lg A)$ antibody titers. In chapter 4, the lessons learned from the coughing rat model of pertussis and implications/uses of the model are explored, as well as avenues to improve vaccine efficacy. The work presented here carefully details a preclinical model that can be used to not only evaluate pathogenesis of $B p$, but can be used to test vaccine efficacy. The data presented further supports that mucosal immunization of DTaP could potentially be a viable option to improve the current DTaP vaccine efficacy. 


\subsection{References}

1. Higgs R, Higgins SC, Ross PJ, Mills KHG. 2012. Immunity to the respiratory pathogen Bordetella pertussis. Mucosal Immunol 5:485-500.

2. Holmes W. 1940. Bacillary and rickettsial infections, acute and chronic.

3. Bordet J, Gengou O. 1906. Le Microbe de la Coqueluche. Les Ann l'Institut Pasteur 20:731-741.

4. Wollstein M. THE BORDET-GENGOU BACILLUS OF PERTUSSIS.'.

5. Heininger U. 2010. Update on pertussis in children. Expert Rev Anti Infect Ther. Expert Rev Anti Infect Ther.

6. Warfel JM, Beren J, Merkel TJ. 2012. Airborne transmission of Bordetella pertussis. J Infect Dis2012/07/19. 206:902-906.

7. Urisu A, Cowell JL, Manclark CR. 1986. Filamentous hemagglutinin has a major role in mediating adherence of Bordetella pertussis to human WiDr cells. Infect Immun 52:695-701.

8. Tuomanen E, Weiss A. 1985. Characterization of two adhesins of Bordetella pertussis for human ciliated respiratory-epithelial cells. J Infect Dis 152:118-125.

9. Van Den Berg BM, Beekhuizen H, Willems RJL, Mooi FR, Van Furth R. 1999. Role of Bordetella pertussis virulence factors in adherence to epithelial cell lines derived from the human respiratory tract. Infect Immun 67:1056-1062.

10. Cherry JD, Brunell PA, Golden GS, Karzon DT. 1988. Report of the Task Force on Pertussis and Pertussis Immunization—1988. Pediatrics 81.

11. Howson CP, Howe CJ, Fineberg H V. 1991. Adverse Effects of Pertussis and Rubella Vaccines.

12. Signs and Symptoms of Whooping Cough (Pertussis) | CDC. 
13. Mattoo S, Cherry JD. 2005. Molecular pathogenesis, epidemiology, and clinical manifestations of respiratory infections due to Bordetella pertussis and other Bordetella subspecies. Clin Microbiol Rev 18:326-382.

14. Tozzi AE, Pastore Celentano L, Ciofi Degli Atti ML, Salmaso S. 2005. Diagnosis and management of pertussis. CMAJ. Canadian Medical Association.

15. Feng Y, Chiu CH, Heininger U, Hozbor DF, Tan TQ, von König CHW. 2021. Emerging macrolide resistance in Bordetella pertussis in mainland China: Findings and warning from the global pertussis initiative. Lancet Reg Heal - West Pacific. Elsevier Ltd.

16. Briere EC, Pondo TH, Schmidt M, Skoff T, Shang N, Naleway A, Martin S, Jackson ML. 2018. Assessment of Tdap Vaccination Effectiveness in Adolescents in Integrated Health-Care Systems. J Adolesc Heal 62:661-666.

17. Yeung KHT, Duclos P, Nelson EAS, Hutubessy RCW. 2017. An update of the global burden of pertussis in children younger than 5 years: a modelling study. Lancet Infect Dis 17:974-980.

18. Pinto M V., Merkel TJ. 2017. Pertussis disease and transmission and host $r$ esponses: insights from the baboon model of pertussis. J Infect 74:S114-S119.

19. Uhl MA, Miller JF. 1994. Autophosphorylation and phosphotransfer in the Bordetella pertussis BvgAS signal transduction cascade. Proc Natl Acad Sci U S A 91:1163-1167.

20. Uhl MA, Miller JF. 1996. Central role of the BvgS receiver as a phosphorylated intermediate in a complex two-component phosphorelay. J Biol Chem 271:3317633180. 
21. Uhl MA, Miller JF. 1996. Integration of multiple domains in a two-component sensor protein: The Bordetella pertussis BvgAS phosphorelay. EMBO J 15:10281036.

22. Melvin JA, Scheller E V., Miller JF, Cotter PA. 2014. Bordetella pertussis pathogenesis: Current and future challenges. Nat Rev Microbiol2014/03/13. 12:274-288.

23. Merkel TJ, Stibitz S, Keith JM, Leef M, Shahin R. 1998. Contribution of regulation by the bvg locus to respiratory infection of mice by Bordetella pertussis. Infect Immun 66:4367-4373.

24. Scarlato V, Aricò B, Prugnola A, Rappuoli R. 1991. Sequential activation and environmental regulation of virulence genes in Bordetella pertussis. EMBO J 10:3971-5.

25. Moon K, Bonocora RP, Kim DD, Chen Q, Wade JT, Stibitz S, Hinton DM. 2017. The BvgAS regulon of Bordetella pertussis. MBio 8.

26. Hegerle N, Rayat L, Dore G, Zidane N, Bedouelle H, Guiso N. 2013. In-vitro and in-vivo analysis of the production of the Bordetella type three secretion system effector A in Bordetella pertussis, Bordetella parapertussis and Bordetella bronchiseptica. Microbes Infect 15:399-408.

27. Emsley P, McDermott G, Charles IG, Fairweather NF, Isaacs NW. 1994. Crystallographic characterization of pertactin, a membrane-associated protein from Bordetella pertussis. J Mol Biol 235:772-773.

28. Relman DA, Domenighini M, Toumanen E, Rappuoli R, Falkow S. 1989. Filamentous hemagglutinin of Bordetella pertussis: Nucleotide sequence and 
crucial role in adherence. Proc Natl Acad Sci U S A 86:2637-2641.

29. Williamson YM, Moura H, Whitmon J, Woolfitt AR, Schieltz DM, Rees JC, Guo S, Kirkham H, Bouck D, Ades EW, Tondella ML, Carlone GM, Sampson JS, Barr JR. 2015. A Proteomic Characterization of Bordetella pertussis Clinical Isolates Associated with a California State Pertussis Outbreak . Int J Proteomics 2015:112.

30. Mooi FR, Jansen WH, Brunings $\mathrm{H}$, Gielen $\mathrm{H}$, van der Heide HGJ, Walvoort HC, Guinee PAM. 1992. Construction and analysis of Bordetella pertussis mutants defective in the production of fimbriae. Microb Pathog 12:127-135.

31. Kimura A, Mountzouros KT, Relman DA, Falkow S, Cowell JL. 1990. Bordetella pertussis filamentous hemagglutinin: Evaluation as a protective antigen and colonization factor in a mouse respiratory infection model. Infect Immun 58:7-16.

32. Geuijen CA, Willems RJ, Bongaerts M, Top J, Gielen H, Mooi FR. 1997. Role of the Bordetella pertussis minor fimbrial subunit, FimD, in colonization of the mouse respiratory tract. Infect Immun 65:4222-8.

33. Guevara C, Zhang C, Gaddy JA, lqbal J, Guerra J, Greenberg DP, Decker MD, Carbonetti N, Starner TD, McCray PB, Mooi FR, Gómez-Duarte OG. 2016. Highly differentiated human airway epithelial cells: a model to study host cell-parasite interactions in pertussis. Infect Dis (London, England) 48:177-88.

34. Robinson A, Ashworth LAE, Irons LI. 1989. Serotyping Bordetella pertussis strains. Vaccine. Vaccine.

35. Carbonetti NH. 2010. Pertussis toxin and adenylate cyclase toxin: Key virulence factors of Bordetella pertussis and cell biology tools. Future Microbiol 5:455-469. 
36. Witvliet MH, Burns,' DL, Brennan,' MJ, Poolman JT, Manclark' CR. 1989. Binding of Pertussis Toxin to Eucaryotic Cells and Glycoproteins INFECTION AND IMMUNITY.

37. Wong WSF, Rosoff PM. 1996. Pharmacology of pertussis toxin B-oligomer. Can J Physiol Pharmacol 74:559-564.

38. Saukkonen K, Burnette WN, Mar VL, Masure HR, Tuomanen El. 1992. Pertussis toxin has eukaryotic-like carbohydrate recognition domains. Proc Natl Acad Sci U S A 89:118-122.

39. Stein PE, Boodhoo A, Armstrong GD, Heerze LD, Cockle SA, Klein MH, Read RJ. 1994. Structure of a pertussis toxin-sugar complex as a model for receptor binding. Nat Struct Biol 1:591-596.

40. Carbonetti NH, Artamonova G V, Van Rooijen N, Ayala VI. 2007. Pertussis toxin targets airway macrophages to promote Bordetella pertussis infection of the respiratory tract. Infect Immun 75:1713-1720.

41. Andreasen C, Carbonetti NH. 2009. Role of neutrophils in response to Bordetella pertussis infection in mice. Infect Immun2008/12/24. 77:1182-1188.

42. Morse SI, Morse JH. 1976. Isolation and properties of the leukocytosis- and lymphocytosis- promoting factor of Bordetella pertussis. J Exp Med 143:14831502.

43. Gregg KA, Merkel TJ. 2019. Pertussis Toxin: A key component in pertussis vaccines? Toxins (Basel). MDPI AG.

44. Carbonetti NH. 2016. Bordetella pertussis: New concepts in pathogenesis and treatment. Curr Opin Infect Dis. Lippincott Williams and Wilkins. 
45. Sebo P, Osicka R, Masin J. 2014. Adenylate cyclase toxin-hemolysin relevance for pertussis vaccines. Expert Rev Vaccines 13:1215-1227.

46. Wang X, Maynard JA. 2015. The Bordetella adenylate cyclase repeat-in-toxin (RTX) domain is immunodominant and elicits neutralizing antibodies. J Biol Chem 290:3576-3591.

47. Ladant D, Ullmann A. 1999. Bordetella pertussis adenylate cyclase: a toxin with multiple talents. Trends Microbiol 7:172-176.

48. Ehrmann IE, Gray MC, Gordon VM, Gray LS, Hewlett EL. 1991. Hemolytic activity of adenylate cyclase toxin from Bordetella pertussis. FEBS Lett 278:79-83.

49. Nash ZM, Cotter PA. 2019. Bordetella filamentous hemagglutinin, a model for the two partner secretion pathway. Microbiol Spectr 7.

50. Gray MC, Donato GM, Jones FR, Kim T, Hewlett EL. 2004. Newly secreted adenylate cyclase toxin is responsible for intoxication of target cells by Bordetella pertussis. Mol Microbiol 53:1709-1719.

51. Masin J, Osicka R, Bumba L, Sebo P. 2015. Bordetella adenylate cyclase toxin: A unique combination of a pore-forming moiety with a cell-invading adenylate cyclase enzyme. Pathog Dis. Oxford University Press.

52. Ladants D, Michelsong S, Sarfatill R, Gillesll A-M, Predeleanus R, Barzus O. 1989. Characterization of the Calmodulin-binding and of the Catalytic Domains of Bordetella pertussis Adenylate Cyclase.

53. Hewlett EL, Donato GM, Gray MC. 2006. Macrophage cytotoxicity produced by adenylate cyclase toxin from Bordetella pertussis: More than just making cyclic AMP! Mol Microbiol 59:447-459. 
54. Skopova K, Tomalova B, Kanchev I, Rossmann P, Svedova M, Adkins I, Bibova I, Tomala J, Masin J, Guiso N, Osicka R, Sedlacek R, Kovar M, Sebo P. 2017. Cyclic AMP-elevating capacity of adenylate cyclase toxin-hemolysin is sufficient for lung infection but not for full virulence of Bordetella pertussis. Infect Immun 85.

55. Khelef N, Guiso N. 1995. Induction of macrophage apoptosis by Bordetella pertussis adenylate cyclase-hemolysin. FEMS Microbiol Lett 134:27-32.

56. Kamanova J, Kofronova O, Masin J, Genth H, Vojtova J, Linhartova I, Benada O, Just I, Sebo P. 2008. Adenylate Cyclase Toxin Subverts Phagocyte Function by RhoA Inhibition and Unproductive Ruffling. J Immunol 181:5587-5597.

57. Smith AM, Guzmán CA, Walker MJ. 2001. The virulence factors of Bordetella pertussis: A matter of control. FEMS Microbiol Rev 25:309-333.

58. lida T, Okonogi T. 1971. Lienotoxicity of Bordetella pertussis in mice. J Med Microbiol 4:51-61.

59. Pertussis | Surveillance Trend Reporting and Case Definition | CDC.

60. Klein NP. 2014. Licensed pertussis vaccines in the United States. Hum Vaccin Immunother 10:2684-2690.

61. Kendrick PL. 1943. A field study of alum-precipitated combined pertussis vaccine and diphtheria toxoid for active immunization. Am J Epidemiol 38:193-202.

62. Kendrick PLD, With FAPHA, Analyses S, Weiss ES. Use of Alum-Treated Pertussis Vaccine, and of Alum-Precipitated Combined Pertussis Vaccine and Diphtheria Toxoid, for Active Immunization*.

63. Libster R, Edwards KM. 2012. Re-emergence of pertussis: what are the solutions? Expert Rev Vaccines 11:1331-1346. 
64. Kendrick PL. 1975. Can Whooping Cough Be Eradicated? J Infect Dis 132:707712.

65. Cody CL, Baraff LJ, Cherry JD, Marcy SM, Manclark CR. 1981. Nature and Rates of Adverse Reactions Associated with DTP and DT Immunizations in Infants and Children. Pediatrics 68.

66. BYERS RK, MOLL FC. 1948. ENCEPHALOPATHIES FOLLOWING PROPHYLACTIC PERTUSSIS VACCINE. Pediatrics 1.

67. Sato Y, Kimura M, Fukumi H. 1984. Development of a pertussis component vaccine in Japan. Lancet (London, England) 1:122-6.

68. Noble GR, Bernier RH, Esber EC, Hardegree MC, Hinman AR, Klein D, Saah AJ. 1987. Acellular and whole-cell pertussis vaccines in Japan. Report of a visit by US scientists. JAMA 257:1351-6.

69. Kimura M. 1991. Japanese clinical experiences with acellular pertussis vaccines. Dev Biol Stand 73:5-9.

70. Edwards KM, Meade BD, Decker MD, Reed GF, Rennels MB, Steinhoff MC, Anderson EL, Englund JA, Pichichero ME, Deloria MA, Deforest A. 1995. Comparison of 13 Acellular Pertussis Vaccines: Overview and Serologic Response. Pediatrics 96:548-557.

71. Hill HA, Elam-Evans LD, Yankey D, Singleton JA, Dietz V. 2016. Vaccination Coverage Among Children Aged 19-35 Months - United States, 2015. MMWR Morb Mortal Wkly Rep 65:1065-1071.

72. National, State, and Local Area Vaccination Coverage Among Children Aged 1935 Months - United States, 2011. 
73. Klein NP, Bartlett J, Fireman B, Baxter R. 2016. Waning Tdap Effectiveness in Adolescents. Pediatrics.

74. Klein NP, Bartlett J, Rowhani-Rahbar A, Fireman B, Baxter R. 2012. Waning Protection after Fifth Dose of Acellular Pertussis Vaccine in Children. N Engl J Med 11367:1012-9.

75. Klein NP, Bartlett J, Fireman B, Rowhani-Rahbar A, Baxter R. 2013. Comparative Effectiveness of Acellular Versus Whole-Cell Pertussis Vaccines in Teenagers. Pediatrics 131:e1716-e1722.

76. Sheridan SL, Frith K, Snelling TL, Grimwood K, Mclntyre PB, Lambert SB. 2014. Waning vaccine immunity in teenagers primed with whole cell and acellular pertussis vaccine: recent epidemiology. Expert Rev Vaccines 13:1081-1106.

77. Sheridan SL, Ware RS, Grimwood K, Lambert SB. 2012. Number and order of whole cell pertussis vaccines in infancy and disease protection. JAMA - J Am Med Assoc. American Medical Association.

78. Tartof SY, Lewis M, Kenyon C, White K, Osborn A, Liko J, Zell E, Martin S, Messonnier NE, Clark TA, Skoff TH. 2013. Waning Immunity to Pertussis Following 5 Doses of DTaP. Pediatrics2013/03/13. 131:e1047-e1052.

79. Weigand MR, Peng Y, Loparev V, Batra D, Bowden KE, Burroughs M, Cassiday PK, Davis JK, Johnson T, Juieng P, Knipe K, Mathis MH, Pruitt AM, Rowe L, Sheth M, Tondella ML, Williams MM. 2017. The History of Bordetella pertussis Genome Evolution Includes Structural Rearrangement. J Bacteriol 199:e00806-16.

80. Williams MM, Sen KA, Weigand MR, Skoff TH, Cunningham VA, Halse TA, Tondella ML. 2016. Bordetella pertussis strain lacking pertactin and pertussis 
toxin. Emerg Infect Dis https://doi.org/10.3201/eid2202.151332.

81. Barkoff A-M, Mertsola J, Pierard D, Dalby T, Hoegh SV, Guillot S, Stefanelli P, van Gent M, Berbers G, Vestrheim D, Greve-Isdahl M, Wehlin L, Ljungman M, Fry NK, Markey K, He Q. 2019. Pertactin-deficient Bordetella pertussis isolates: evidence of increased circulation in Europe, 1998 to 2015. Euro Surveill 24.

82. Pertussis | Surveillance Trend Reporting and Case Definition | CDC.

83. Weigand MR, Peng Y, Loparev V, Batra D, Bowden KE, Burroughs M, Cassiday PK, Davis JK, Johnson T, Juieng P, Knipe K, Mathis MH, Pruitt AM, Rowe L, Sheth M, Lucia Tondella M, Williams MM, Weigand CM, Anke Becker E. 2017. The History of Bordetella pertussis Genome Evolution Includes Structural Rearrangement. jb.asm.org $1 \mathrm{~J}$ Bacteriol 199:806-822.

84. Weigand MR, Williams MM, Peng Y, Kania D, Pawloski LC, Tondella ML. 2018. Genomic Survey of Bordetella pertussis Diversity, United States, 2000-2013. Emerg Infect Dis 25:780-783.

85. Mooi FR. 2010. Bordetella pertussis and vaccination: The persistence of a genetically monomorphic pathogen. Infect Genet Evol 10:36-49.

86. Kallonen T, Gröndahl-Yli-Hannuksela K, Elomaa A, Lutyńska A, Fry NK, Mertsola J, He Q. 2011. Differences in the genomic content of Bordetella pertussis isolates before and after introduction of pertussis vaccines in four European countries. Infect Genet Evol 11:2034-2042.

87. Octavia S, Maharjan RP, Sintchenko V, Stevenson G, Reeves PR, Gilbert GL, Lan R. 2011. Insight into Evolution of Bordetella pertussis from Comparative Genomic Analysis: Evidence of Vaccine-Driven Selection. Mol Biol Evol 28:707-715. 
88. Sealey KL, Harris SR, Fry NK, Hurst LD, Gorringe AR, Parkhill J, Preston A. 2014. Genomic Analysis of Isolates From the United Kingdom 2012 Pertussis Outbreak Reveals That Vaccine Antigen Genes Are Unusually Fast Evolving https://doi.org/10.1093/infdis/jiu665.

89. Otsuka N, Han H-J, Toyoizumi-Ajisaka H, Nakamura Y, Arakawa Y. 2012. Prevalence and Genetic Characterization of Pertactin-Deficient Bordetella pertussis in Japan. PLoS One 7:31985.

90. Elahi S, Holmstrom J, Gerdts V. 2007. The benefits of using diverse animal models for studying pertussis. Trends Microbiol. Trends Microbiol.

91. Kendrick PL, Eldering G, Dixon MK, Misner J. 1947. Mouse Protection Tests in the Study of Pertussis Vaccine: A Comparative Series Using the Intracerebral Route for Challenge. Am J Public Heal Nations Heal 37:803-810.

92. STANDFAST AF. 1958. The comparison between field trials and mouse protection tests against intranasal and intracerebral challenges with Bordetella pertussis. Immunology 1:135-143.

93. Guiso N, Capiau C, Carletti G, Poolman J, Hauser P. 1999. Intranasal murine model of Bordetella pertussis infection. I. Prediction of protection in human infants by acellular vaccines. Vaccine1999/07/07. 17:2366-2376.

94. Sato Y, Izumiya,' ' K, Sato H, Cowell JL, Manclarkw CR. 1980. Aerosol Infection of Mice with Bordetella pertussis INFECTION AND IMMUNITY.

95. PROOM H. 1947. The immunological aspects of experimental Haemophilus pertussis infection. J Pathol Bacteriol 59:165-180.

96. Elahi S, Brownlie R, Korzeniowski J, Buchanan R, O'connor B, Peppler MS, 
Halperin SA, Lee SF, Babiuk LA, Gerdts V. 2005. Infection of Newborn Piglets with Bordetella pertussis: a New Model for Pertussis. Infect Immun 73:3636-3645.

97. Elahi S, Thompson DR, Strom S, O'Connor B, Babiuk LA, Gerdts V. 2008. Infection with Bordetella parapertussis but not Bordetella pertussis causes pertussis-like disease in older pigs. J Infect Dis 198:384-392.

98. Elahi S, Buchanan RM, Attah-Poku S, Townsend HGG, Babiuk LA, Gerdts V. 2006. The host defense peptide beta-defensin 1 confers protection against Bordetella pertussis in newborn piglets. Infect Immun 74:2338-2352.

99. Elahi S, Buchanan RM, Babiuk LA, Gerdts V. 2006. Maternal immunity provides protection against pertussis in newborn piglets. Infect Immun 74:2619-2627.

100. Weiss AA, Hewlett EL. 1986. Virulence factors of Bordetella pertussis. Annu Rev Microbiol. Annual Reviews 4139 El Camino Way, P.O. Box 10139, Palo Alto, CA 94303-0139, USA .

101. Warfel JM, Beren J, Kelly VK, Lee G, Merkel TJ. 2012. Nonhuman primate model of pertussis. Infect Immun2012/01/19. 80:1530-1536.

102. Culotta CS, Harvey DF, Gordon EF. 1935. Whooping cough. II. Experimental study. J Pediatr 6:743-752.

103. Huang CC, Chen PM, Kuo JK, Chiu WH, Lin ST, Lin HS, Lin YC. 1962. Experimental Whooping Cough. N Engl J Med 266:105-111.

104. Lin T-M. 1958. Experimental Whooping Cough in Monkey. Formos Med Assoc.

105. SAUER LW. 1929. EXPERIMENTAL WHOOPING COUGH. Arch Pediatr Adolesc Med 37:732.

106. Shibley GS. 1934. Etiology of Whooping Cough. Proc Soc Exp Biol Med 31:576- 
579.

107. Warfel JM, Zimmerman LI, Merkel TJ. 2014. Acellular pertussis vaccines protect against disease but fail to prevent infection and transmission ina nonhuman primate model. Proc Natl Acad Sci U S A 111:787-792.

108. Warfel JM, Merkel TJ. 2013. Bordetella pertussis infection induces a mucosal IL17 response and long-lived Th17 and Th1 immune memory cells in nonhuman primates. Mucosal Immunol 6:787-796.

109. Warfel JM, Zimmerman LI, Merkel TJ. 2016. Comparison of three whole-cell pertussis vaccines in the baboon model of pertussis. Clin Vaccine Immunol 23:4754.

110. Jiang W, Wei C, Mou D, Zuo W, Liang J, Ma X, Wang L, Gao N, Gu Q, Luo P, Ma Y, Li J, Liu S, Shi L, Sun M. 2021. Infant rhesus macaques as a non-human primate model of Bordetella pertussis infection. BMC Infect Dis 21:1-12.

111. Hornibrook JW, Ashburn LL. 1939. A Study of Experimental Pertussis in the Young Rat. Public Heal Reports 54:439.

112. Woods DE, Franklin R, Cryz SJ, Ganss M, Peppler M, Ewanowich C, Ewanowich C. 1989. Development of a rat model for respiratory infection with Bordetella pertussis. Infect Immun 57:1018-1024.

113. Hornibrook JW. 1939. Cultivation of Phase I H. pertussis in a Semisynthetic Liquid Medium. Public Heal Reports 54:1847.

114. Nakamura K, Shinoda N, Hiramatsu Y, Ohnishi S, Kamitani S, Ogura Y, Hayashi T, Horiguchi Y. 2019. BspR/BtrA, an Anti- $\sigma$ Factor, Regulates the Ability of Bordetella bronchiseptica To Cause Cough in Rats. mSphere 
https://doi.org/10.1128/msphere.00093-19.

115. Wardlaw AC, Hall E, Parton R. 1993. Coughing rat model of pertussis. Biologicals 21:27-29.

116. Hall E, Parton R, Wardlaw AC. 1994. Cough production, leucocytosis and serology of rats infected intrabronchially with Bordetella pertussis. J Med Microbiol 40:205213.

117. Hall E, Parton R, Wardlaw AC. 1999. Time-course of infection and responses in a coughing rat model of pertussis. J Med Microbiol 48:95-98.

118. Parton R, Hall E, Wardlaw AC. 1994. Responses to Bordetella pertussis mutant strains and to vaccination in the coughing rat model of pertussis. J Med Microbiol 40:307-312.

119. Hall E, Parton R, Wardlaw AC. 1997. Differences in coughing and other responses to intrabronchial infection with Bordetella pertussis among strains of rats. Infect Immun 65:4711-4717.

120. Hall E, Parton R, Wardlaw AC. 1998. Responses to acellular pertussis vaccines and component antigens in a coughing-rat model of pertussis. Vaccine 16:1595603.

121. Janda WM, Santos E, Stevens J, Celig D, Terrile L, Schreckenberger PC. 1994. Unexpected isolation of Bordetella pertussis from a blood culture. J Clin Microbiol. American Society for Microbiology.

122. Mahon BP, Sheahan BJ, Griffin F, Murphy G, Mills KH. 1997. Atypical disease after Bordetella pertussis respiratory infection of mice with targeted disruptions of interferon-gamma receptor or immunoglobulin mu chain genes. J Exp Med 
$186: 1843-51$.

123. Byrne P, McGuirk P, Todryk S, Mills KHG. 2004. Depletion of NK cells results in disseminating lethal infection with Bordetella pertussis associated with a reduction of antigen-specific Th1 and enhancement of Th2, but not Tr1 cells. Eur J Immunol 34:2579-2588.

124. Dunne PJ, Moran B, Cummins RC, Mills KHG. 2009. CD11C + CD8 $\alpha+$ Dendritic Cells Promote Protective Immunity to Respiratory Infection with Bordetella pertussis . J Immunol 183:400-410.

125. Paddock CD, Sanden GN, Cherry JD, Gal AA, Langston C, Tatti KM, Wu K, Goldsmith CS, Greer PW, Montague JL, Eliason MT, Holman RC, Guarner J, Shieh W, Zaki SR. 2008. Pathology and Pathogenesis of Fatal Bordetella pertussis Infection in Infants. Clin Infect Dis 47:328-338.

126. Carbonetti NH, Artamonova G V., Van Rooijen N, Ayala VI. 2007. Pertussis Toxin Targets Airway Macrophages To Promote Bordetella pertussis Infection of the Respiratory Tract. Infect Immun 75:1713-1720.

127. Higgins SC, Jarnicki AG, Lavelle EC, Mills KHG. 2006. TLR4 Mediates VaccineInduced Protective Cellular Immunity to Bordetella pertussis: Role of IL-17Producing T Cells. J Immunol 177:7980-7989.

128. Mahon BP, Mills KHG. 1999. Interferon-y mediated immune effector mechanisms against Bordetella pertussis, p. 213-217. In Immunology Letters. Immunol Lett.

129. Barbic J, Leef MF, Burns DL, Shahin RD. 1997. Role of gamma interferon in natural clearance of Bordetella pertussis infection. Infect Immun 65:4904-4908.

130. Ryan M, Murphy G, Gothefors L, Nilsson L, Storsaeter J, Mills KHG. 1997. 
Bordetella pertussis respiratory infection in children is associated with preferential activation of type $1 \mathrm{~T}$ helper cells. J Infect Dis 175:1246-1250.

131. Mascart F, Verscheure V, Malfroot A, Hainaut M, Piérard D, Temerman S, Peltier A, Debrie A-S, Levy J, Del Giudice G, Locht C. 2003. Bordetella pertussis Infection in 2-Month-Old Infants Promotes Type 1 T Cell Responses . J Immunol 170:15041509.

132. Mc Guirk P, Mahon BP, Griffin F, Mills KHG. 1998. Compartmentalization of T cell responses following respiratory infection with Bordetella pertussis: hyporesponsiveness of lung T cells is associated with modulated expression of the co-stimulatory molecule CD28. Eur J Immunol 28:153-163.

133. Mcguirk P, Mills KHG. 2000. A regulatory role for interleukin 4 in differential inflammatory responses in the lung following infection of mice primed with Th1- or Th2- inducing pertussis vaccines. Infect Immun 68:1383-1390.

134. Harvill ET, Cotter PA, Miller JF. 1999. Pregenomic comparative analysis between Bordetella bronchiseptica RB50 and Bordetella pertussis Tohama I in murine models of respiratory tract infection. Infect Immun1999/10/26. 67:6109-6118.

135. Confer DL, Eaton JW. 1982. Phagocyte impotence caused by an invasive bacterial adenylate cyclase. Science (80- ) 217:948-950.

136. Eby JC, Gray MC, Hewlett EL. 2014. Cyclic AMP-mediated suppression of neutrophil extracellular trap formation and apoptosis by the Bordetella pertussis adenylate cyclase toxin. Infect Immun2014/10/08. 82:5256-5269.

137. Mills KH, Barnard A, Watkins J, Redhead K. 1993. Cell-mediated immunity to Bordetella pertussis: role of Th1 cells in bacterial clearance in a murine respiratory 
infection model. Infect Immun 61:399-410.

138. Granström M, Hanngren K, Olinder-Nielson AM, Holmblad P, Mark A. 1991. Specific immunoglobulin for treatment of whooping cough. Lancet 338:1230-1233.

139. Cherry JD, Gornbein J, Heininger U, Stehr K. 1998. A search for serologic correlates of immunity to Bordetella pertussis cough illnesses. Vaccine 16:19016.

140. Mills KHG, Ryan M, Ryan E, Mahon BP. 1998. A murine model in which protection correlates with pertussis vaccine efficacy in children reveals complementary roles for humoral and cell- mediated immunity in protection against Bordetella pertussis. Infect Immun 66:594-602.

141. Leef M, Elkins KL, Barbic J, Shahin RD. 2000. Protective immunity to Bordetella pertussis requires both $\mathrm{B}$ cells and $\mathrm{CD} 4(+) \mathrm{T}$ cells for key functions other than specific antibody production. J Exp Med 191:1841-1852.

142. Ross PJ, Sutton CE, Higgins S, Allen AC, Walsh K, Misiak A, Lavelle EC, McLoughlin RM, Mills KH. 2013. Relative contribution of Th1 and Th17 cells in adaptive immunity to Bordetella pertussis: towards the rational design of an improved acellular pertussis vaccine. PLoS Pathog 9:e1003264.

143. Storsaeter J, Hallander HO, Gustafsson L, Olin P. 1998. Levels of anti-pertussis antibodies related to protection after household exposure to Bordetella pertussis. Vaccine https://doi.org/10.1016/S0264-410X(98)00227-8.

144. van der Lee S, Hendrikx LH, Sanders EAM, Berbers GAM, Buisman AM. 2018. Whole-cell or acellular pertussis primary immunizations in infancy determines adolescent cellular immune profiles. Front Immunol 9:51. 
145. Ryan M, Murphy G, Ryan E, Nilsson L, Shackley F, Gothefors L, Øymar K, Miller E, Storsaeter J, Mills KH. 1998. Distinct T-cell subtypes induced with whole cell and acellular pertussis vaccines in children. Immunology 93:1-10.

146. Ausiello CM, Urbani F, la Sala A, Lande R, Cassone A. 1997. Vaccine- and antigen-dependent type 1 and type 2 cytokine induction after primary vaccination of infants with whole-cell or acellular pertussis vaccines. Infect Immun 65:216874.

147. Kapil P, Merkel TJ. 2019. Pertussis vaccines and protective immunity. Curr Opin Immunol 59:72-78.

148. Redhead K, Watkins J, Barnard A, Mills KHG. 1993. Effective immunization against Bordetella pertussis respiratory infection in mice is dependent on induction of cell-mediated immunity. Infect Immun 61:3190-3198.

149. Warfel JM, Zimmerman LI, Merkel TJ. 2014. Acellular pertussis vaccines protect against disease but fail to prevent infection and transmission in a nonhuman primate model. Proc Natl Acad Sci 111:787-792.

150. Brummelman J, Wilk MM, Han WGH, van Els CACM, Mills KHG. 2015. Roads to the development of improved pertussis vaccines paved by immunology. Pathog Dis. Oxford Academic.

151. Wendelboe AM, Van Rie A, Salmaso S, Englund JA. 2005. Duration of Immunity Against Pertussis After Natural Infection or Vaccination. Pediatr Infect Dis J 24:S58-S61.

152. Boehm DT, Wolf MA, Hall JM, Wong TY, Sen-Kilic E, Basinger HD, Dziadowicz SA, Gutierrez M de la P, Blackwood CB, Bradford SD, Begley KA, Witt WT, Varney 
ME, Barbier M, Damron FH. 2019. Intranasal acellular pertussis vaccine provides mucosal immunity and protects mice from Bordetella pertussis. npj Vaccines 4.

153. Wolf MA, Boehm DT, DeJong MA, Wong TY, Sen-Kilic E, Hall JM, Blackwood CB, Weaver KL, Kelly CO, Kisamore CA, Bitzer GJ, Bevere JR, Barbier M, Damron FH. 2021. Intranasal Immunization with Acellular Pertussis Vaccines Results in Long-Term Immunity to Bordetella pertussis in Mice https://doi.org/10.1128/IAI.00607.

154. Skerry CM, Mahon BP. 2011. A live, attenuated Bordetella pertussis vaccine provides long-term protection against virulent challenge in a murine model. Clin Vaccine Immunol2010/12/08. 18:187-193.

155. Lin A, Apostolovic D, Jahnmatz M, Liang F, Ols S, Tecleab T, Wu C, van Hage M, Solovay K, Rubin K, Locht C, Thorstensson R, Thalen M, Loré K. 2020. Live attenuated pertussis vaccine BPZE1 induces a broad antibody response in humans. J Clin Invest 130.

156. Maurer H, Höfler K, Hilbe W, Huber E. 1979. Preliminary findings with oral whooping cough vaccination in young infants. Wien Hlin Wochenschr.

157. Baumann E, Binder BR, Falk W. 1985. Development and clinical use of an oral heat-inactivated whole cell pertussis vaccine. Dev Biol Stand VOL. 61:511-516.

158. Guzman CA, Brownlie RM, Kadurugamuwa J, Walker MJ, Timmis KN. 1991. Antibody responses in the lungs of mice following oral immunization with Salmonella typhimurium aroA and invasive Escherichia coli strains expressing the filamentous hemagglutinin of Bordetella pertussis. Infect Immun 59:4391-4397.

159. KASUGA T, NAKASE Y, UKISHIMA K, TAKATSU K. 1954. Studies on 
Haemophilus pertussis. V. Relation between the phase of bacilli and the progress of the whooping-cough. Kitasato Arch Exp Med 27:57-62.

160. Boinett CJ, Harris SR, Langridge GC, Trainor EA, Merkel TJ, Parkhill J. 2015. Complete Genome Sequence of Bordetella pertussis D420. Genome Announc 3. 


\section{Chapter 2. Re-investigating the coughing rat model of pertussis to understand Bordetella pertussis pathogenesis}




\section{Re-investigating the coughing rat model of pertussis to understand Bordetella pertussis pathogenesis}

Jesse M. Hall ${ }^{1,2}$, Jason Kang ${ }^{1,2}$, Sophia M. Kenney ${ }^{1,2}$, Ting Y. Wong ${ }^{1,2}$, Graham J. Bitzer $^{1,2}$, Claire O. Kelly ${ }^{1,2}$, Caleb A. Kisamore ${ }^{1,2}$, Dylan T. Boehm ${ }^{1,2,}{ }^{*}$, Megan A. DeJong ${ }^{1,2}$, M. Allison Wolf ${ }^{1,2}$, Emel Sen-Kilic ${ }^{1,2}$, Alexander M. Horspool ${ }^{1,2}$, Justin R Bevere $^{1,2}$, Mariette Barbier ${ }^{1,2}$, \& F. Heath Damron ${ }^{1,2, \#}$

${ }^{1}$ Department of Microbiology, Immunology and Cell Biology, School of Medicine, West Virginia University, Morgantown, WV

${ }^{2}$ Vaccine Development Center, WVU Health Sciences Center, Morgantown, WV

Running title: Coughing rat model of pertussis

\#Address correspondence to F. Heath Damron, fdamron@hsc.wvu.edu

*Present address: Vaccine and Gene Therapy Institute at Oregon Health Sciences University, 18 Beaverton, Oregon, USA 


\subsection{Abstract}

Bordetella pertussis $(B p)$ is a highly contagious bacterium that is the causative agent of whooping cough (pertussis). Currently, acellular pertussis vaccines (aP; DTaP; Tdap) are used to prevent pertussis disease. However, it is clear that the aP vaccine efficacy quickly wanes, resulting in the re-emergence of pertussis. Furthermore, recent work performed by the CDC suggest that current circulating strains are genetically distinct from strains of the past. Emergence of genetically diverging strains combined with waning aP vaccine efficacy call for re-evaluation of current animal models of pertussis. In this study, we used the rat model of pertussis to compare two genetically divergent strains Tohama 1 and D420. We intranasally challenged seven-week-old Sprague-Dawley rats with $10^{8}$ viable Tohama 1 and D420 and measured the hallmark signs/symptoms of $B p$ infection such as neutrophilia, pulmonary inflammation, and paroxysmal cough using whole body plethysmography. Onset of cough occurred between 2-4 days after $B p$ challenge averaging five coughs per fifteen minutes, with peak coughing occurring at day eight post infection averaging upward of thirteen coughs per fifteen minutes. However, we observed an increase of coughs in rats infected with clinical isolate D420 through 12 days post challenge. The rats exhibited increased bronchial restriction following $B p$ infection. Histology of the lung and flow cytometry confirm both cellular infiltration and pulmonary inflammation. D420 infection induced higher production of anti-Bp IgM antibodies compared to Tohama 1 infection. The coughing rat model provides a way of characterizing disease manifestation differences between $B p$ strains. 


\subsection{Introduction}

Whooping cough (pertussis) is a respiratory disease that is caused by the bacterium Bordetella pertussis (Bp). Pertussis is characterized by severe leukocytosis, bronchopneumonia, hypoglycemia, and paroxysmal cough $(1,2)$. During the catarrhal stage, $B p$ colonizes the upper respiratory epithelium. Once colonization occurs, $B p$ releases toxins such as pertussis toxin (PT), adenylate cyclase toxin (ACT) as well as others. Following the catarrhal stage is the paroxysmal stage that is characterized by severe coughing episodes. Innate and adaptive immune responses result in clearance of the bacterium allowing for the patient or host to convalescence. Despite extensive research, a full understanding of $B p$ pathogenesis remains elusive for reasons such as: vaccine pressure, lack of predictive models, and strain evolution (3). One of the major reasons for this gap of knowledge is the lack of a suitable animal model that shares common disease symptoms as seen in humans. Besides non-human primates, rats are the only other known model of pertussis that share similar coughing manifestation as seen in humans (4). As of now, the origin or the trigger of cough has yet to be determined (5). The rat model of pertussis offers a potential animal model that can be used to evaluate Bp pathogenesis.

In 1938, Hornibrook and Ashburn were the first to report that rats infected with $B p$ induced cough-like paroxysms and bronchopneumonia as seen in humans (6). Hornibrook and Ashburn found that young rats were capable of being infected and the bacterium could be cultured from the lungs (6). Infected rats produced coughs that could be heard from a distance of 20 feet (6). Twenty-six of the thirty-one infected rats that did not succumb to 
the infection had pathology indicative of inflammation in the lungs, most notably early neutrophil infiltration followed by recruitment of mononuclear cells (6). The subsequent studies utilized intrabronchial inoculation of $B p$ encased in agar beads and confirmed leukocytosis and paroxysmal cough by sound activated tape recorders from 5 to 21 days post challenge $(7,8)$. Further development of the rat model led to the evaluation of PT negative strains and the evaluation of coughs (9). Strain BP357, which is deficient in PT resulted in low cough induction (9). Further evaluation of the rat model demonstrated leukocytosis, weight loss, and paroxysmal cough in Sprague-Dawley rats during the course of $B p$ infection (10). During the development phase of the acellular pertussis vaccine $(\mathrm{aP}, \mathrm{DTaP})$, the rat model was used to test vaccine efficacy of various acellular pertussis formulations and was used to validate protection against the onset of leukocytosis and cough (11). Recently, an intranasal rat model has been used to evaluate bacterial factors responsible for cough in Bordetella bronchiseptica (Bb) (12). BspR ,which is an anti-sigma factor of $B b$, plays some role in cough induction upon infection (12). Collectively, these studies show that the pertussis rat model can be used to critically evaluate $B p$ pathogenesis and disease progression.

In the 1940s, whole-cell pertussis (wP and DTP) vaccines were introduced to protect against $B p$ infection (13). Widespread use of DTP in the United States led to a $90 \%$ decrease in the number of reported $B p$ infections (13). Despite the efficacy of this vaccine, serious adverse side-effects ensued, leading to the development of acellular pertussis vaccines. The antigens that are included in the current aPs contain: filamentous hemagglutinin (FHA), fimbriae (FIM), PT, and pertactin (PRN). After the switch from the 
$w P$ to the aPs, there has been a significant increase in the number of pertussis cases in the US and Europe (14). While nationwide vaccine coverage is $95 \%$ in the United States, the incidence of $B p$ infections rose in the past 10 years as the number of aP vaccinated only population has increased in size. (15). While vaccine coverage remains high, the population dynamics are changing, and more people are aP immunized as the new generations are born and as the WP only generations age. Numerous studies have demonstrated waning efficacy of aPs in parallel with the emergence of genetically divergent strains of $B p(3,16-21)$. Increased surveillance of $B p$ has led to the identification of clinical isolates that do not express PRN, FIM, and even PT the hallmark toxin of the organism (22). One plausible hypothesis to explain these observations is that circulating $B p$ strains have evolved due to acellular vaccine pressure. While we know the current strains are genetically different, we do not know if this genetic variability affects virulence, disease burden, toxicity, or fitness of the pathogen (3).

Early rat studies were performed with intranasal administration of $B p$ for infection (6). Subsequent rat challenge studies used $B p$ encased in agar beads for intrabronchial instillation $(7-11,23)$. While the agar bead infection method was successful at establishing infection, we aimed to utilize the simplicity of intranasal administration. We sought to re-investigate the coughing rat model of pertussis to compare the pathogenesis of reference strain Tohama 1 to the recent clinical isolate D420, which has been extensively studied in the baboon model of pertussis (24-30). Tohama 1 was first isolated from a case of whooping cough in 1954, while D420 was isolated in 2002 from a critically ill infant in Texas $(25,31)$. Although Tohama 1 is now a reference strain that has been 
widely used since Sato and Sato developed the aP but recent data has shown that this strain is an considered an outlier $(32,33)$. Tohama 1 does expresses PRN but has lower expression of PT and ACT (34). When Tohama 1 was used as a challenge strain in baboons, the baboons did not exhibit symptoms of pertussis despite being infected (26). This led to the selection of recent clinical isolate D420 as the baboon challenge strain, which readily infected and caused disease (26). With 48 years of potential genetic divergence, we sought to understand the differences in pathogenesis between these two commonly studied strains. D420 is known to infect mice and baboons and it belongs to the clade (CDC013) of strains that represented $50 \%$ of isolates recovered in the US in 2000 (3). However, it is important to note that D420 has an intact pertactin gene and it does express the PRN protein as confirmed by shotgun proteomic analysis (data not shown).

In our current study, we aimed to re-establish an upper respiratory tract infection model in rats following intranasal challenge with Tohama I and D420. We hypothesized that recent isolate D420 would induce a more severe disease profile compared to Tohama 1 , as previous research investigating pathogenesis in rhesus macaques noted that Tohama 1 infected animals did not exhibit overt disease symptoms (26). Sprague-Dawley rats were intranasally challenged with $B p$ and we characterized their disease progression profile over a twelve-day infection. Cough was critically assessed utilizing whole body plethysmography (WBP). Bacterial colonization, leukocytosis, and serological responses were measured as a result of infection. Rats challenged with D420 had increased coughing, greater bacterial burden in the respiratory tract, and a more robust IgM antibody 
response compared to Tohama 1 . The coughing rat model of pertussis can shed light on the pathogenesis of $B p$ and will likely be useful tool for pertussis vaccine evaluation.

\subsection{Results}

D420 infected rats have increase number of coughs compared to strain Tohama 1 over the entire course of infection.

Over the past decade it is became clear that improvements to the acellular pertussis vaccine strategy are needed due to the rise of PRN mutants, genomic divergence, and epidemiological data. We believe in order to improve pertussis vaccines we need to better understand the pathogenesis of $B p$ in an animal model that shares similar clinical manifestation of pertussis as seen in humans. Here, we intranasally infected sevenweek-old Sprague Dawley rats with $10^{8} \mathrm{CFU}$ of Bp strain D420 or Tohama 1 (Fig. 5) in an effort establish infection and observe $B p$ induced cough. For negative controls (no bacterial challenge), rats were intranasally administered sterile phosphate buffer saline (PBS). Paroxysmal cough is the hallmark symptom of pertussis and is thought to play a major role in transmission of the organism to a new host. To quantify respiratory function during infection, we utilized whole body plethysmography (WBP). WBP instrumentation consists of specialized containment chambers which monitor box flow, temperature, and airflow changes to measure respiratory function. Counting coughs via WBP provides an extremely accurate and unbiased way of counting coughs based on cough waveforms. Rat containment chambers were placed inside a laminar flow hood and connected to a computer that would monitor the rats breathing (Fig. S1). Early studies reported that $B p$ infection in young rats induced coughs that were audible by ear (6) and in our preliminary 
studies this was apparent to us as well (data not shown). Studies in the 1990s, of paroxysmal cough in rats were quantified with an analog sound recording device (7). We hypothesized that rats infected with recent isolate D420 would induce more coughs than reference strain Tohama 1, because rhesus macaques infected with Tohama 1 did not exhibit overt disease symptoms but D420 induced robust coughing in baboons (26). Bp infected rats developed cough at days 2-11 (Fig. 6B-C), while the mock infected rats (Fig. 6A) only had a few isolated coughs, unrelated to infection. Days 1-3 post infection, the average cough count of rats infected with Tohama 1 or D420 was less then 5 coughs per fifteen minutes. The average cough count doubled by day 7 post infection with peak coughing occurring at day 8 post infection with an average of 13 coughs per fifteen minutes of monitoring. After day 8 , the average cough count for rats infected with D420 remained above 10 coughs per fifteen minutes, while the rats infected with Tohama 1 averaged less then 5 coughs. To summarize the cough data, the average cough count over the course of infection is shown (Fig. 6D). We observed a significant increase in the number of coughs at day eight post infection of $B p$ infected rats compared to the mock challenge control. Rats infected with D420 coughed a total of 949 times, whereas rats infected with Tohama 1 coughed a total of 724 times over the entire twelve-day infection. To analyze differences in cough count between strains, we utilized Area Under the Curve (AUC) analysis (Fig. 6E). AUC analysis allows us to quantify the number of coughs over the entire course of infection per rat. Upon this analysis, we note a significant increase in the number of coughs for rats infected with D420 compared to the mock challenged rats over the twelve-day infection, while there was no significant difference observed in Tohama 1 infected rats compared to mock challenge (non-infected). 


\section{Bp infection causes pulmonary distress.}

Infection with $B p$ leads to mucus production, lung damage, and invasion of cellular infiltrates into the bronchioles of the lung (35). The lungs of infants with $B p$ exhibit edema, necrotizing bronchiolitis, and inflammation in the lung leading to respiratory distress (35). WBP was used to quantify the pulmonary distress over the course of infection. Pulmonary distress was measured by calculating the enhanced pause (PenH) of the animal (Fig. 7). The higher the PenH value, the increased respiratory distress of the animal. PenH corresponds with the resistance associated with peak inspiratory height (PIF) and peak expiratory height (PEF) and taking into account time between early and late expiration per breath (Fig. S2). With increased constriction, the expiratory peak becomes prominent and the area of the (PEF) becomes a larger percentage of the complete expiratory area. Others factors that contribute to the increased PenH is a decrease in relaxation time $(\mathrm{Tr})$ in relation to expiratory time (Te). Previous research has shown that severe inflammation in the lung of mice directly correlated with an increase in PenH (36). More recently, SARSCoV-2 infected mice were observed to have subtle but significant increase in PenH post challenge $(37,38)$. Compared to the mock challenged rats both D420 and Tohama 1 infected rats had an increase in PenH (Fig. 7). We observed a significant increase at day 9 with rats infected with D420 and at day 6 with rats infected with Tohama 1 compared to mock challenge.

\section{Histological assessment of the lung and nasal cavity}

To further evaluate the inflammation in the lung, we utilized histology to confirm both acute and chronic inflammation in response to $B p$ infection. At days 1, 3, 6, 9, and 12 post 
challenge the left lobes were collected, sectioned, and stained with hematoxylin and eosin (H\&E) (Fig. 8A\&C). Whole-lung images were taken to assess cellular infiltration (Fig. S3). Once stained, the blinded slides were then scored by a board-certified pathologist. Mock challenge animals exhibited minimal inflammatory infiltrates consisting of focal accumulations of mononuclear cells in the parenchyma and occasional infiltrates of neutrophils surrounding blood vessels (Fig. 8A). Rats infected with Tohama 1 and D420 had significant increase in their acute inflammation scores compared to mock challenge animals at day 1 and 3 post infection (Fig. 8B). Day 1 post infection had the highest acute inflammation score (Fig. 8B). Rats exhibited mild to moderate neutrophil infiltration of the parenchyma, blood vessels, and the bronchioles (Fig. 8A). Markers of acute inflammation resolved after day 6 post challenge; however, infected rats at day 9 exhibited obvious differences in the lung associated with mild to moderate infiltration of mononuclear cells and higher chronic inflammation compared to mock challenge animals (Fig. 8C). At day 12 post infection, we notice moderate resolution of inflammation in both D420 and Tohama 1 infected rats (Fig. 8B\&D). We also observed a significant increase in lung weight, which can also be associated with elevated inflammation, at days 1 and 9 post challenge in D420 infected rats compared to the mock challenge control (Fig. 9A). At day 12 post challenge rats infected with Tohama 1 had a significant increase in lung weight compared to the mock challenge rats (Fig. 9A). These results confirm that $B p$ infection induces inflammation in the lung of coughing Sprague-Dawley rats.

Due to the fact that $B p$ is a mucosal pathogen, we sought out to investigate any phenotypic changes or inflammation in the Nasal-Associated Lymphoid Tissue (NALT) of 
pertussis infected rats via histology. NALT helps elicit immunity against airborne and mucosal pathogens (39). The NALT is located above the hard palate and studies have also shown that with the introduction of antigen via vaccination or bacterial components, cellular expansion of the NALT ensues (40-44). The NALT was stained with H\&E, and we observed an increase in cellular infiltrate and a size enlargement in the NALTs of $B p$ infected rats compared to the mock challenged (Fig. 10A). ImageJ analysis was used to measure the area of both the left and right NALT of each animal (Fig. 10B). This data could mark the potential cellular expansion of the NALT upon infection with Bp. Further analysis of the expanding cell populations in the NALT will warrant more analysis especially in the context of vaccine immunity.

\section{D420 infected rats have increased bacterial burden in the respiratory tract.}

We determined bacterial burden in the respiratory tract for Tohama I and D420 challenged rats at days $1,3,6,9$, and 12 . Over the coughing timeline, we see an overall decrease in viable bacteria in the lung, trachea, and nasal cavity. In the lung, we observed a significant increase in the recovered bacteria in the D420 infected rats compared to the Tohama 1 infected rats at both days 1 and 3 (Fig. 11A). The same trend is also seen in the nasal cavity at day 1 post infection (Fig. 11C). We observed no difference in the viable bacteria recovered in the trachea (Fig. 11B). Previous pertussis studies utilizing the rat model of pertussis noted weight loss from intrabronchial infection of Bp strain 18-323 (10). $B p$ infection is sufficient to cause weight loss in rats (10). To confirm this using intranasal infection, body weight was measured before challenge and upon euthanasia to calculate percent weight change. We observed a significant decrease in weight gain in rats infected 
with $B p$ strain D420 and Tohama 1 compared to the mock challenged at day 12 post infection (Fig. 9B-D).

Next, we aimed to compare the bacterial colonization between outbred mice and rats in an effort to relatively compare the bacterial burden between models. We utilized the bacterial burden data in CD1 mice and compared them to our data in rats (45). To address the body weight differences between the mice and rats, we used the CFUs/organ and divided them by their respective body weight for a crude comparison. Our data suggests that despite having a higher initial challenge dose in the rat, the bacterial burden remaining in the respiratory tract is higher in a mouse compared to the rat later during infection (Fig. S4). In general, the rat model is likely a lower challenge dose per body weight model of pertussis compared to mouse models.

\section{Immunofluorescence confirms colonization of $B p$ in the lungs and nasal cavity.}

We next wanted to assess the location of bacterial colonization in the nasal cavity and visualize any bacteria left behind following flushing of the nares. We sectioned the skulls after flushing the nasal cavity and utilized Immunofluorescence (IF). After visually scanning the nasal cavity with confocal microscopy, we found bacteria in the nasal turbinates and the NALT (Fig. 12A\&C). We also confirmed that the bacteria were found between the epithelial cells (Fig. 12B). The relative locations of where the bacteria were found in the nasal cavity can be seen in (Fig. S5A). Our results show that $B p$ remained in the nasal cavity over the entire course of infection even after flushing the nares (Fig. S5B). The flushing of the nasal cavity underrepresents the total number of $B p$ in the nasal cavity. In the lung, Bp colonizes the bronchioles, while the alveolar spaces remain non- 
colonized (Fig. 12D\&F). IF was performed on the nasal cavity and lung of mock challenge animals to access non-specific binding (Fig. S6). To assess any potential differences between the strains we blindly manually counted the labeled microcolonies in the lung and nasal cavity. It is also worthy to note, we found no differences in microcolony counts in the lung or nasal cavity between the strains (Fig. S5 C-D) despite our observed differences in bacterial burden by CFU enumeration. CFU is likely a better indicator of overall bacterial burden, but IF revealed that some $B p$ remains in the airway following flushing of the nares.

\section{Measurement of recruitment of neutrophils in response to $B p$ infection}

Previous studies utilizing the rat model of pertussis noted leukocytosis and an increase in the amount of total white blood cells using agar-encased $B p$ for infection (8). Neutrophils circulating through the blood were measured during intranasal infection by antibody staining and flow cytometry, as $B p$ infection results in varying amounts of

neutrophilia $(35,46)$. One caveat of analyzing cell populations via flow cytometry in the rats is the limited availability of rat specific antibodies compared to mice. Recent research by Barnett-Vanes et al has developed a flow cytometry panel to analyze cell populations in a model of LPS-induced pulmonary inflammation (47). The percentage of CD45+ (CD161- B220- CD43+ His48Hi) neutrophils in the blood were assessed in Bp infected rats compared to the mock challenged animals (Fig. 13A). Specifically, a significant increase in the number of circulating neutrophils in the blood of rats infected with D420 at days 1 and 9 , with Tohama 1 infected rats only having a significant increase to mock challenge at day 9 (Fig. 13A). Next, cytokines concentrations in the lung and serum were measured to identify factors that could contribute to the recruitment of neutrophils, 
specifically IL-6 and IL-17. IL-6 is a potent inducer of Th17 polarization and been found elevated during murine infection with $B p(45,48-50)$. Surprisingly, we did not observe any significant changes in IL-6 due to $B p$ infection (Fig. 13B\&C). It has been well documented in the mouse model that the production of IL-17 in pertussis plays a role in the increase of circulating neutrophils (51). There were no significant changes in IL-17a in the serum or lungs of $B p$ infected rats compared to mock challenge (Fig. 13D\&E). Th1 and Th2 cytokines and chemokines in the lung and serum were also measured (Fig. S7Fig. S10). We did not observe an increase in the induction of cytokines by infection overall; however, in the serum, rats infected with D420 had a significant increase in IL-5 compared to Tohama 1 infected rats at day 12 post infection (Fig. S7). There was a significant increase in IL-5 and TNF-alpha at day 6 post infection in the lung in rats infected with Tohama 1 compared to D420 (Fig. S8). We also observed significant increases in chemokines MIP-1a, RANTES, MCP-3, MIP-2, and IP10 in rats infected with D420 at day 12 post challenge in the serum compared to rats infected with Tohama 1 (Fig. S19). In the lung, MIP-2 and IP10 were increased in rats infected with D420 compared to mock challenge control (Fig. S10). Despite slight differences highlighted above we did not observe a massive difference in the cytokine and chemokine responses during infection.

\section{Serological analysis of $B p$ specific antibodies}

Enzyme-linked immunosorbent assays (ELISA) were performed to determine the serological response generated from $B p$ infection. We measured IgM and IgG antibody titers in the serum to whole bacterium and $B p$ associated virulence factors at days 1,3 , 6,9 , and 12 post infection. In previous studies utilizing the rat model, intrabronchial 
infection induced the generation of $\lg G$ antibody titers to sonicated $B p 28$ days post infection (8). Intranasal infection of strain D420 has generated significant anti-PT IgG antibody titers in the baboon model at days 17-19 of infection (26). However, analysis of antibody titers generated against an intranasal pertussis infection in the rat has yet to be determined and we wanted to characterize the primary antibody response to $B p$ (Fig. S11). We observed a significant 10 -fold increase in the anti-Bp IgM antibody response at day 9 and an almost 20 -fold increase 12 days post challenge in rats infected with D420 compared to mock challenge, while we also saw a significant 6 -fold increase in anti-Bp IgM at day 12 post infection to the amount of anti-Bp IgM from Tohama 1 infection (Fig. 14A). At day 12 post infection, we observed that $B p$ infection induced significant production of anti-Bp IgG in the serum compared to mock challenge animals (Fig. 14F). At day 12 post infection, Tohama 1 infected rats had a significant increase, nearly double anti-Bp IgG antibody titers compared to rats infected with D420. AUC analysis revealed a significant increase in anti-Bp IgM antibodies from rats infected with D420 compared to Tohama 1 infected rats (Fig. S12A). However, no difference was observed in AUC of the anti-Bp IgG antibody response over the total course of infection between the bacterial challenge groups (Fig. S12B). It is also important to note we also did not see a significant increase in the generation of antibodies titers to PT, ACT, PRN, and FHA in Bp infected rats compared to mock challenge infected animals (Fig. 14B-E, G-J). We would also like to note that $\lg \mathrm{A}$ antibodies were not detected to either whole bacteria or PT in the lung and nasal cavity at days 1 and 9 post challenge (data not shown). 


\subsection{Discussion}

To date, the only known reservoir for $B p$ is humans, making the development of a suitable animal model challenging. Multiple models have been studied to recapitulate similar disease symptoms seen in humans, as well as provide insight into the pathogenesis of $B p$. We set out to re-investigate the rat model of pertussis that was originally introduced in 1938 (6). In our study, we have confirmed that intranasal administration of Bp led to bacterial colonization of the upper and lower respiratory tract (Fig. 11). Through intranasal instillation, bacteria were able to infect the nasal cavity, trachea, and lung post challenge. This is important to note as infants with pertussis experience pneumonia more frequently than adolescents and adults (35). Mouse models of pertussis also rely on depositing large numbers of bacteria into the lung to establish infection (2). Here, our data shows that through intranasal infection, we establish an infection of both the upper and lower respiratory tract, resembling the infection seen in adolescents and adults with pertussis $(52,53)$.

Previous reports have shown $B p$ infection of rats induced paroxysmal coughs, and these coughs were counted by analog audio recordings (7). Utilizing WBP we are able to precisely and digitally count coughs and measure respiratory capacity for the first time with rats infected with $B p$ (Fig. 6\&7). Here, coughs induced from infection appeared at day 1-2 post challenge, with the number of coughs gradually increasing with peak cough occurring at day 8 post challenge. Rats challenged with intrabronchial instillation of $B p$ noted similar peak cough counts approximately 10 days post challenge (54). This is also one of the first studies utilizing WBP to count coughs in rats with bacterial infection. We did observe some infected rats that did not overtly cough. This reflects the frequency of 
coughs in adolescents and adults (55). Previous studies using baboons also show that $B p$ infection elicits severe coughs lasting over 2 weeks post challenge with peak coughs per hour being at day 4 post challenge (26). It is also important to note that at peak coughing, the bacterial burden was low, which was also observed in previous $B p$ infected rat research (7). The data suggest that other factors could be playing a role in cough production despite the bacterial infection being resolved. Given that $B p$ infected rats coughed, we also investigated transmission of $B p$ from infected 7-week-old rats (actively coughing) cohoused with more susceptible 3-week-old rats and we have observed no evidence of transmission via negative qPCR (IS481) analysis of swabs from the nasal cavity and no active coughing in the naïve 3-week-old rats. We also performed a challenge study with Bb strain KM22 in 7-week-old Sprague Dawley rats, and challenged rats coughed violently and earlier after bacterial challenge compared to $B p$ infected rats. $B b$ infected rats coughed the most when the bacterial loads in the respiratory tract were at their highest, which could potentially lead to transmission of the bacterium. TLR4 deficient rats could be investigated to evaluate transmission of $B p(56)$. We have also observed that the increased bronchi restriction coincides with the increased coughs during days 6-11 post challenge (Fig. 7). The decreased lung capacity and increase in cough could be further associated with an increase of chronic inflammation in the lung (Fig. 8). Our data suggests that the coughing rat model of pertussis can be used to potentially evaluate the mechanism of cough, which still requires further investigation.

Initial studies characterizing the serum antibody response to rats infected with $B p$ noted an increase antibody response to endotoxin correlated with the low amount of cultivable 
organisms in the lung following intrabronchial challenge (7). Hall et al. demonstrated that rats infected with $B p$ become sera positive to whole cell $B p$ lysate, FHA, and PT at 28 days post intrabronchial challenge (8). Anti-FHA antibody titers were not detected until day 16 post challenge, while anti-PT antibody titers were not detected until day 28 post challenge (23). In our study, we characterized the antibody response following intranasal challenge of $B p$ over the whole infection timeline. Using $B p$ coated ELISA plates, we detected anti-Bp IgG antibody titers in rats infected with $B p$ at day 9 post challenge with a significant increase compared to mock challenge observed at day 12 (Fig. 14). We also measured a significant increase in anti-Bp IgM antibody titers in rats infected with D420 compared to the mock challenge and Tohama 1 infected animals (Fig. 14). One potential hypothesis that could explain this observation is the genetic differences between the two strains as D420 harbors the ptxP3 allele, leading to an increase in PT production (25). PT has been shown to suppress $B p$ antibody responses in the serum and limit expression of antigen presenting receptors (57-60).

With the increased interest in developing new pertussis vaccines, it is imperative for the development of animal models to evaluate the pathogenic potential of current emerging strains, as well as identify any potential differences in disease burden between genetically divergent strains. Sato and Sato first used $B p$ strain Tohama 1 in the development of the acellular pertussis vaccine, and Tohama 1 is still being used in pertussis research today, notably due to its genetic malleability (61). D420 has been instrumental in the development of the baboon model and has also been used to study vaccine efficacy in regards to transmission in the baboon model (29). We hypothesize that the observed differences could be due to the increased amount of PT through the ptxP3 allele of D420 
(25). Strains, such as D420, harboring the $p t x P 3$ allele lead to increase in virulence, PT production, and prevalence $(62,63)$. A previous study utilizing the intranasal mouse model has shown an increase in bacterial colonization with ptxP3 strains (64). Parton et al. illustrated that infection with a PT deficient strain was unable to induce cough (9). It is possible that there are other factors beyond PT that can account for the observed virulence differences between Tohama I and D420. In the rat model, unlike the mouse, we also did not measure any significant increases in IL-17 and IL-6 in the lung and serum following challenge. IL-17 and IL-6 are increased following $B p$ infection in mice and baboon $(30,48,65)$. Further investigation is needed to explain the observed differences. One potential approach to evaluate differences between strains is the use of a neonatal rat model of pertussis. Studying $B p$ in neonates may be useful in distinguishing subtle differences between strains. Hornibrook et al demonstrated that $B p$ infected young 3 week rats had $65 \%$ mortality, while 7 week old rats do not succumb to infection with similar dose (6).

In summary, we have demonstrated that the rat model of pertussis can be used as a tool to further study $B p$ pathogenesis and recapitulate some of the similar symptoms of pertussis as seen in humans. Pertussis is often described as a 100 day cough, because adolescents and adults extended duration of coughing (66). Coughing episodes in infants with pertussis can lead to vomiting, choking, gagging, and apneic episodes that can lead to seizures (67). Mice have not been used to evaluate coughing manifestation in pertussis until recently, and baboons are expensive and limited to specialized facilities (68). The coughing rat of pertussis provides feasibility through low husbandry costs, availability of 
animals, and ease of use as a model. The immunological tools available in murine research is vast but as basic research continue to evolve, so will the resources and immunological tools available for rats. Recently flow cytometry markers have been developed to study innate immune cells, and adaptive immune cells such as $B$ and $T$ cells (47). In other studies, we have ELISpots assays to investigate antigen specific immune populations as well. Increasing availability of antibodies has also allowed for investigating systemic and mucosal serological responses. Future studies utilizing the coughing rat model of pertussis will capitalize on the growing immunological toolbox in regard to vaccination against pertussis.

In this study, we carefully compared two established strains that have been used in the pertussis field, Tohama 1 and D420. In future studies, we aim to evaluate whole cell and acellular vaccine mediated immunity against D420. Armed with this model, we can further our understanding of pathogenesis, host response during pertussis, genetic divergence between strains, and vaccine-mediated immunity.

\subsection{Materials and methods}

Bordetella pertussis strains and growth conditions. Bp strain Tohama 1 was graciously provided by Dr. Peter Sebo (Czech Academy of Sciences) and strain D420 was acquired from the CDC Pertussis Lab provided by Drs. Maria L. Tondella and Michael Weigand. Bp strain Tohama 1 and D420 were cultured on Bordet Gengou (BG) agar (Remel ${ }^{\mathrm{TM}}$ Cat. R45232) that was supplemented with $15 \%$ defibrinated sheep blood (Hemostat Laboratories Cat. DSB500) (69). Bacteria were cultured on BG plates for 48 hrs at $36^{\circ} \mathrm{C}$. Bp was transferred from BG plates with polyester swabs (Puritan Cat. 22- 
029-574) and transferred into $20 \mathrm{ml}$ Stainer-Scholte liquid media (SSM) in new $125 \mathrm{ml}$ flasks (Thermo Fisher Scientific Cat. FB500125) (70). The liquid cultures were grown for $24 \mathrm{hrs}$ at $36^{\circ} \mathrm{C}$ inside a shaking incubator at $180 \mathrm{rpm}$.

Intranasal challenge with $B$. pertussis. Seven-week-old $\sim 170 \mathrm{~g}$ female SpragueDawley rats (Charles River Cat. 001CD) were used for challenge. Bp was grown as described above. The rats were then anesthetized with ketamine and xylazine 50-100/5$10 \mathrm{mg} / \mathrm{kg}$ and challenged with $10^{8} \mathrm{CFUs}(100 \mu \mathrm{l}$ intranasally), administering two $50 \mu \mathrm{l}$ doses one in each nostril (Fig. 5). Body weight of each rat was recorded before challenge and immediately post euthanasia. Rats were euthanized at day 1, 3, 6, 9, and 12 post bacterial challenge. Mock challenge animals (no bacteria) were administered $100 \mu \mathrm{l}$ of sterile endotoxin free phosphate buffer saline (PBS) (Thermo Fisher Scientific Cat. TMS012A) intranasally. Upon euthanasia blood was collected via cardiac puncture and transferred into ethylenediaminetetraacetic acid (EDTA) (BD Cat. 365974) and serum separation (BD Cat. 026897) tubes. Blood collected in the EDTA tube was used for flow cytometric analysis, while blood collected in the serum separation tubes were used to isolate the serum via centrifugation $(15,000 \times \mathrm{g}$ for $3 \mathrm{~min})$ and used for serological and cytokine analysis. Lung and trachea were excised and homogenized to determine bacterial burden. Upon removal, the wet weight of the lungs was recorded. Lungs were then collected in gentleMACS $C$ tubes (Miltenyi Biotec) and homogenized using gentleMACS ${ }^{\mathrm{TM}}$ Dissociator (Cat. 130-095-927). The trachea was homogenized using a Polytron homogenizer. To determine the bacterial burden in the nares, $2 \mathrm{mls}$ of sterile $1 \mathrm{x}$ PBS was flushed through the nares and collected for plating. Serial dilutions of the 
homogenates and nasal collection were plated on BG plates supplemented with ceftibuten (Sigma-Aldrich Cat. SML0037) $10 \mu \mathrm{g} / \mathrm{ml}$ to decrease growth of normal rat respiratory tract flora.

\section{Analysis of coughing and bronchiole restriction using whole-body} plethysmography. To quantify respiratory function during infection, we utilized a Buxco $®$ FinePointe $^{T M}$ Whole Body Plethysmograph instrument (WBP) (DSI). FinePointe ${ }^{T M}$ software was used for collecting, analyzing, and reporting the breathing data. Everyday post challenge for twelve consecutive days at 5:00PM the rats were placed inside designated chambers to acclimate for $5 \mathrm{~min}$. We chose this time, not only due to rats being nocturnal, but this was the time the animals were most awake and active as confirmed by video camera (data not shown). Following acclimation, the rat's respiratory profile was recorded for $15 \mathrm{~min}$. Each chamber is fitted with a transducer that measures the changes in box flow and airflow of the subject. The chambers are also fitted with a screen pneumotach that allows airflow in and out of the chamber that can be recorded. Coughs were counted during the designated $15 \mathrm{~min}$, and enhanced pause $(\mathrm{PenH})$ was calculated to signify bronchiole restriction. Coughs were counted based on large box flow changes of the subject, and changes in both humidity and temperature of the air flowing in and out of the subject with classical cough-like waveforms. Cough detection algorithm is applied with patented fuzzy logic criteria to determine if the event is a cough (71). When analyzing the number of coughs, each cough in a multi-cough event was counted individually. 
Histological assessment of the lung and nasal-associated lymphoid tissue. Upon euthanasia, the rat skull was excised, and the mandible removed. The skulls were fixed in $10 \%$ formalin (Fisher Scientific Cat. SF98-4) for $48 \mathrm{hrs}$ at $26^{\circ} \mathrm{C}$. After fixation, formalin was removed, and skulls were frozen at $-80^{\circ} \mathrm{C}$ until decalcification. Skulls were decalcified with Richard-Allan Scientific decalcifying solution (Thermo Scientific Ref. 83401) at room temperature for $24-48 \mathrm{hrs}$ and embedded in paraffin. Samples were sectioned and stained with Hematoxylin-Eosin (H\&E). A Biotek Lionheart Fx was used to scan and image the NALT. ImageJ was used to trace and measure the area of both the left and right NALT using the images from the Lionheart Fx. The left lobe of the lung was excised and fixed in $10 \%$ formalin $48 \mathrm{hrs}$ at $26^{\circ} \mathrm{C}$. The left lobe was then embedded in paraffin and stained with H\&E by the WVU Pathology Department. H\&E stained sections were used to characterize and score acute and chronic inflammation of the lung. All scorings were done by a board-certified pathologist (iHisto). Individual scores were based on a standard qualitative scoring criterion: (0 - none, 1 - minimal (rare), 2 - mild (slight), 3 moderate, 4 - marked, 5 -severe). Chronic inflammation was characterized by mononuclear infiltrates of the parenchyma, blood vessels, and airway. Acute inflammation scores were assigned due to the presence of neutrophils in the parenchyma, blood vessels, and airway. All examination and scoring were performed blindly as no knowledge of treatment groups were known.

Imaging of $B p$ in the lung and nasal cavity. Detection of $B p$ in the nasal cavity and the lung were quantified via immunofluorescence (IF) and confocal imaging. The left lobe of the lung and nasal cavity were preserved and sectioned as described above. Sectioned 
samples underwent deparaffination and rehydration using xylene and ethanol $(100 \%-$ $70 \%$ ). Antigen retrieval was performed by incubating samples in citrate buffer at $98^{\circ} \mathrm{C}$ for 20 min. Samples were blocked using 5\% Bovine Serum Albumin (BSA) (Fisher Scientific Cat. 159008) for $1 \mathrm{hr}$ and primarily labeled utilizing a polyclonal rabbit FHA antibody (Gift from Dr. Erik Hewlett) diluted in 1x PBS. Secondary labeling occurred utilizing an antirabbit IgG conjugated with Texas Red (Fisher Scientific Cat. T2767) diluted in 1x PBS. Samples were then covered in mounting media (Prolong Gold Antifade reagent with DAPI Cat. 8961). Samples were imaged using a Nikon A1R confocal microscope. Images were analyzed on DAPI channel and at wavelength $650 \mathrm{~nm}$ for Texas-Red acquisition. Images were acquired using $100 x$ oil immersion lens (100x/1.40 Nikon Plan APO). To identify any potential differences in IF between the two strains, all IF images were deidentified and the microcolonies were manually counted blindly by four volunteers with 2-3 fields of view used per sample.

Flow cytometry analysis of phagocytes. Neutrophil recruitment in the blood was evaluated by flow cytometry. Blood samples upon collection were then lysed with Pharmylse buffer (BD Biosciences Cat. 555899) for 20 min at room temperature, with slight vortexing throughout. The remaining cells were resuspended in RPMI $+10 \% \mathrm{FBS}$ to neutralize the lysis buffer, then centrifuged at $1,000 \mathrm{~g} \times 5$ and washed with the RPMI $+10 \%$ FBS again. Blood was then resuspended in 1\%FBS+PBS+5mM EDTA. Samples were then block with anti-CD32 (BD Pharmingen Cat. 550270) antibody for $30 \mathrm{~min}$ at $4^{\circ} \mathrm{C}$. After incubation, the cells were stained with the appropriate antibody markers; CD45 Alexa flour 700 (Biolegend Cat. 202218), CD161 APC (Biolegend Cat. 205606), CD45R PE Cy7 (eBioscience Cat. 25-0460-82), His48 FITC (eBioscience Cat. 11-0570-82), 
CD43 PE (Biolegend Cat. 202812), and CD3 VioGreen (Miltenyi Biotec Cat. 130-119125) (47). After the addition of antibodies, the cells incubated $1 \mathrm{hr}$ at $4^{\circ} \mathrm{C}$ in the dark. To prepare the lung samples for flow cytometry, the lung homogenate was pushed through a $70 \mu \mathrm{m}$ cell strainer (BioDesign Cell MicroSives Cat. N70R), creating a single-cell suspension. The suspension was centrifuged at $1,000 \times g$ for $5 \mathrm{~min}$. After removal of the supernatant, the pellet was resuspended in Pharmlyse buffer and the cells incubated at $37^{\circ} \mathrm{C}$ for $2 \mathrm{~min}$. After incubation, the cells were centrifuged at $1,000 \times \mathrm{g}$ for $5 \mathrm{~min}$, supernatant was removed and blocked and labeled with antibody as described above. The lung and blood samples were centrifuged at 1,000 $\mathrm{xg}$ for $5 \mathrm{~min}$ and the pellets were resuspended in $0.4 \%$ paraformaldehyde and stored overnight at $4^{\circ} \mathrm{C}$. Samples were washed with 1x PBS and resuspended in 1x PBS for analysis. Cell samples were analyzed on a LSR Fortessa and samples were gated and analyzed using FlowJo v10.

Lung and serum cytokine and chemokine analysis. Lung homogenate samples were centrifuged at $19,000 \times \mathrm{g}$ for $4 \mathrm{~min}$ and the supernatant was removed and stored at $-80^{\circ} \mathrm{C}$. Quantitative analysis of cytokines in the serum and lung homogenate was performed using ProcartaPlex Multiplex Immunoassay kit: Cytokine \& Chemokine 22Plex Rat ProcartaPlex ${ }^{\mathrm{TM}}$ Panel (Cat. EPX220-30122-901) per the manufacturer's instructions.

Serological analysis. Antibody titers of infected rats were measured by ELISA. Bordetella pertussis specific ELISA plates were coated with $50 \mu \mathrm{l}$ of $10^{8}$ B. pertussis grown as mentioned above for infection. For measurement of antibody titers to FHA (Enzo ALX-630-123-0100), PRN (GSK), PT (List Biological Laboratories \#180), and ACT (Gift 
from Dr. Erik Hewlett), we coated plates with $50 \mu \mathrm{l}$ of each antigen at $1 \mu \mathrm{g} / \mathrm{ml}$. Once coated, plates incubated over night at $4^{\circ} \mathrm{C}$. Plates were washed with $1 \times$ PBS-Tween 20 and blocked with $5 \%$ skim milk for 2 hours at $37^{\circ} \mathrm{C}$. Plates were washed again and the serum from the challenge studies were serially diluted down the ELISA plate and incubated for 2 hours at $37^{\circ} \mathrm{C}$. After incubation, the plates were washed and coated with $100 \mu \mathrm{l}$ of secondary goat anti-rat IgG (SouternBiotech Cat. 3030-04) and IgM (SouhternBiotech Cat. 3020-04) antibody at a dilution of 1:2,000 in PBS $+5 \%$ milk. Once coated, the plate was incubated for 1 hour at $37^{\circ} \mathrm{C}$. Plates were then washed again with PBS-Tween 20 and $100 \mu \mathrm{l} p$-nitrophenyl phosphate substate (Thermo Scientific Cat. 37620) was added, prepared by manufactures instructions, and the plate was developed for $30 \mathrm{~min}$ at room temperature for titers to $\lg \mathrm{G}$ and $\lg \mathrm{M}$. Biotek Synergy $\mathrm{H} 1 \mathrm{microplate}$ reader was used to measure the colorimetric signal of the ELISA plate at $A_{450}$. Positive antibody titers were determined by any values that were higher than the baseline. The baseline is set as double the average value of the blank, no serum added to these wells. Generating Nightingale rose plots in Python: Data from individual mice were averaged in Microsoft Excel and log transformed. Values were formatted in Excel to be compatible with Python. Data were imported from .csv files using the pandas package and plotted using the "Barpolar" representation feature in the plotly.graph_objects module. Samples with a titer less than 50 were assigned a value of 0 .

Statistical analysis. All data was analyzed using GraphPad Prism 7. The minimum biological replicates for the challenge studies were four. For statistical comparisons between multiple groups over the entire course of the infection a two-way analysis of 
variance (ANOVA) was used One-way ANOVA was used for comparison between groups for each individual day with Tukey's post hoc test. Unpaired Student $t$-tests were used for area under the curve (AUC) analysis. Follow-up statistical tests are annotated in figure legends.

Data availability. Data requests for figures provided can be addressed to the corresponding author.

Ethics statement. All studies were performed in accordance West Virginia University Institutional Animal Care and Use Committee approved protocol 1811019148.6.

\subsection{Acknowledgements}

The preliminary work on this project was in part supported by the Vaccine Development Center at WVU-HSC through a Research Challenge Grant no. HEPC.dsr.18.6 from the Division of Science and Research, WV Higher Education Policy Commission. The project was also supported by NIH R01Al137155 (F.H.D) and CDC Contract (BAA) 75D301-19-R-67835 (F.H.D). Flow cytometry experiments were performed in the West Virginia University Flow Cytometry Core Facility, which is supported by the National Institutes of Health equipment grant number S10OD016165 and the Institutional Development Award (IDeA) from the National Institute of General Medical Sciences of the National Institutes of Health under grant numbers P30GM103488 (CoBRE) and P20GM103434 (INBRE).

JMH, DTB, and JK performed bacterial challenge. JMH, SMK, and JK monitored rat coughing and respiratory function by whole body plethysmography. All authors 
participated in the animal experiments. JMH and ESK contributed to flow cytometry panel design and analysis. JMH and TYW prepared and ran flow cytometry samples. MAW and MAD performed cytokine analysis. JMH analyzed H\&E histology slides. JMH and GJB performed and analyzed immunohistochemistry. JMH performed ELISA assays. JMH, $\mathrm{MB}$, and FHD contributed to experimental design. JMH wrote manuscript with critical revisions from all authors.

The authors would like to thank Dr. Kathleen Brundage (WVU Flow Cytometry \& Single Cell Core Facility) for assisting in flow cytometry and equipment instruction. The authors would also like to thank Dr. Amanda Ammer (Microscope Imaging Facility) for support in imaging the IF slides. The authors would also like to thank Dr. James Coad, Jacqueline Karakiozis, and Brice Hickey (Pathology/Histology Core Facility) for the preparation of lung and nasal cavity for IF and performing H\&E on the lung and nasal cavity.

The authors would also like to acknowledge that figure 1 was Created with BioRender.com.

\subsection{Figures}




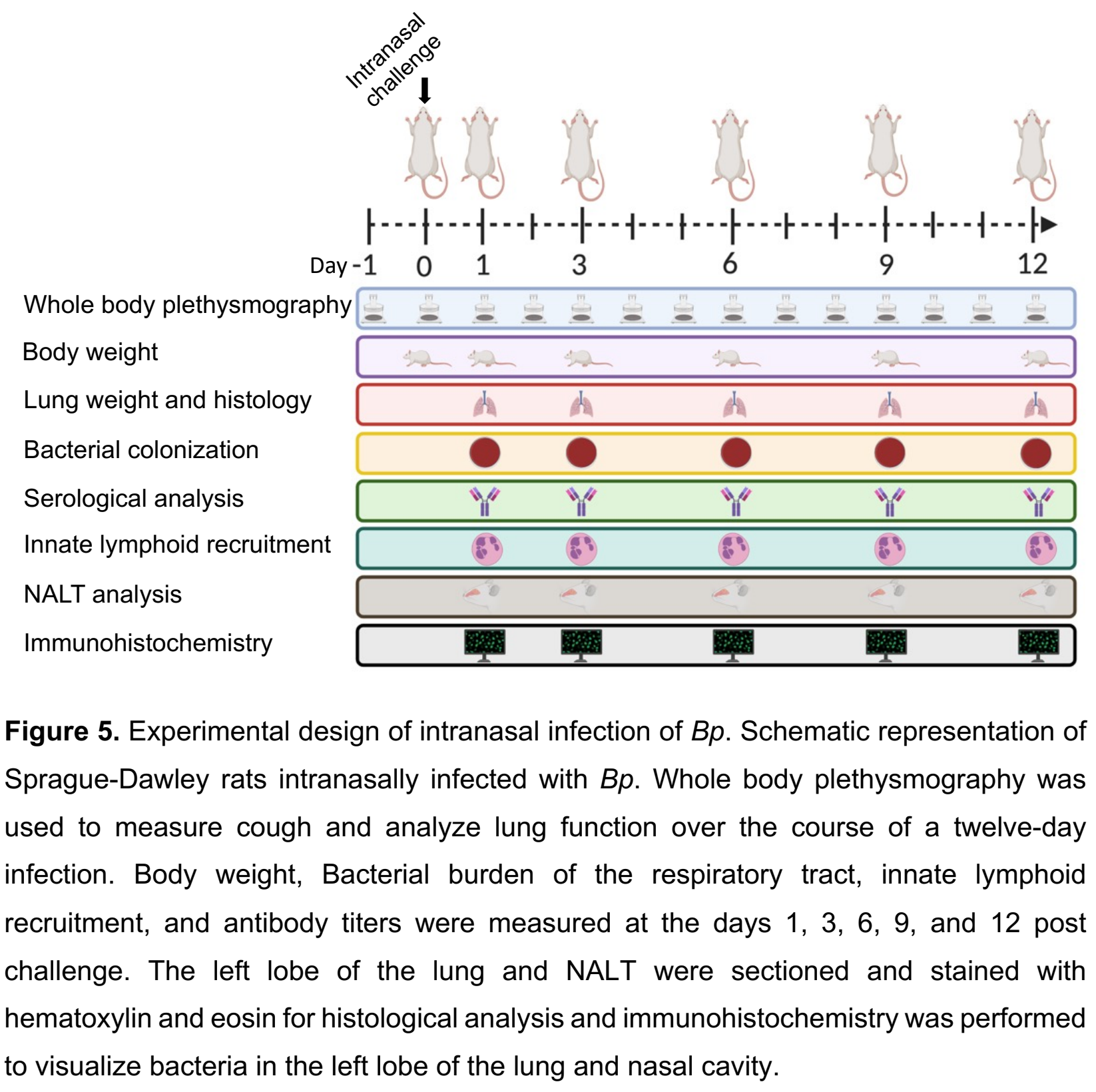


A

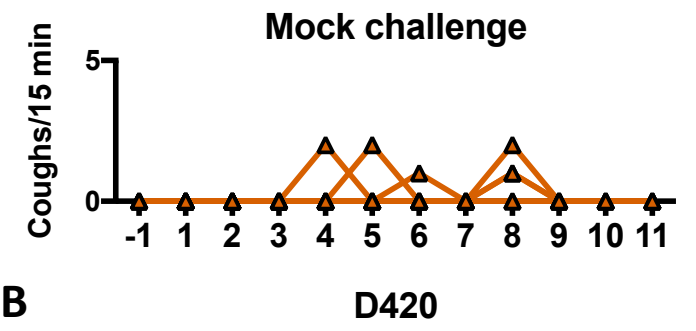

B

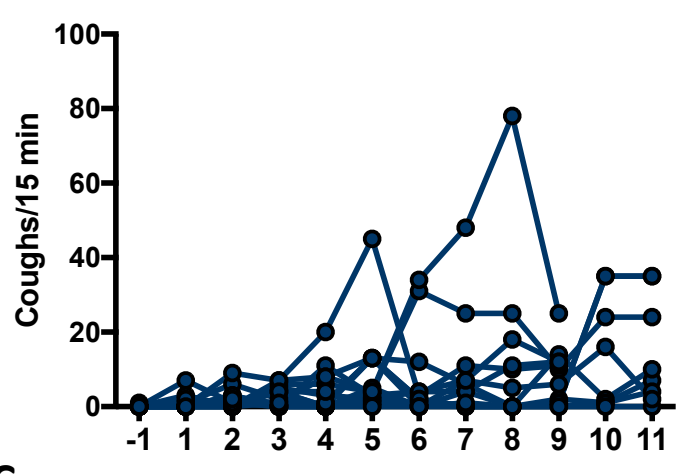

C

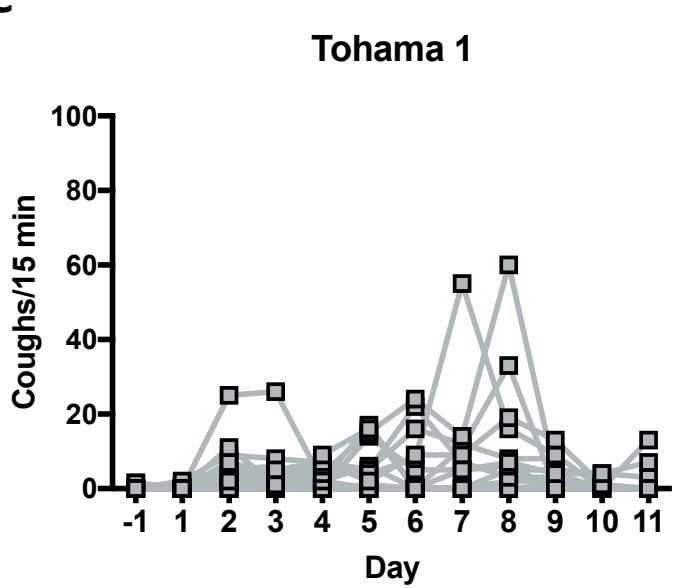

D

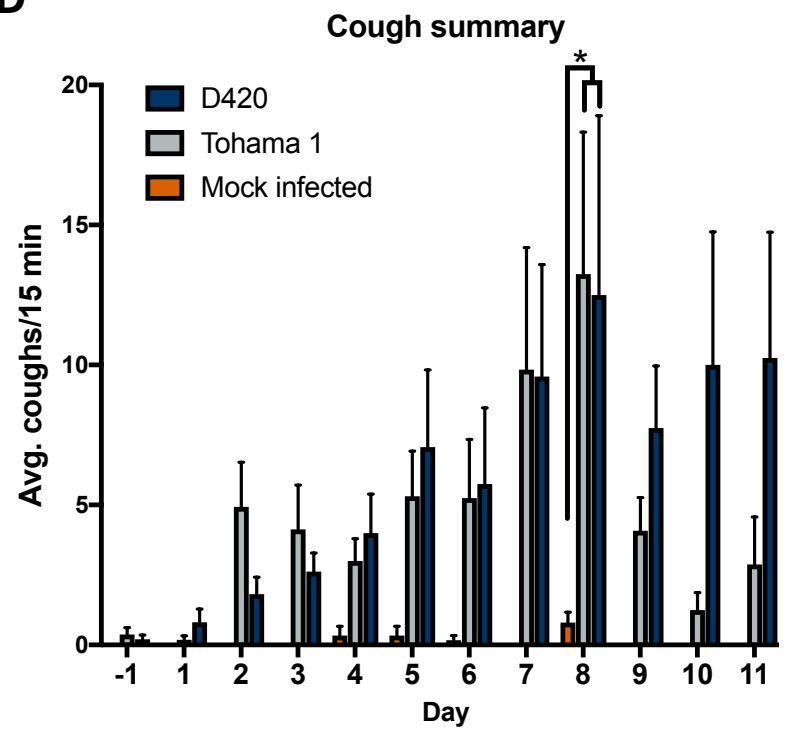

$E$

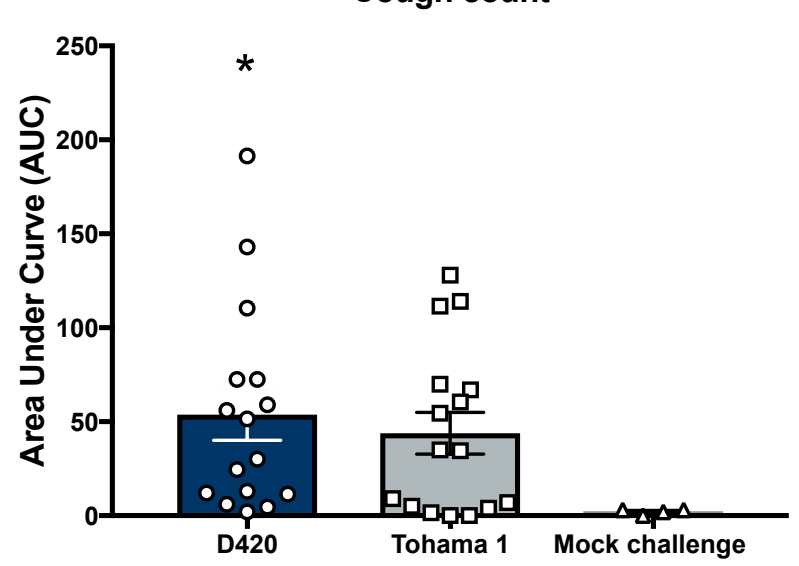

Figure 6. Infection of Bordetella pertussis induces cough in Sprague-Dawley rats. Coughs were measured each day of the twelve-day infection using whole body plethysmography. Coughs were counted for (A) mock challenge rats, (B) rats infected with D420, and (C) rats infected with Tohama 1. (D) Summary of results (A-C) shown as mean \pm SEM $(n=4-8)$. (E) Area under the curve is represented as averages of coughs per fifteen minutes for each rat. $P$ values were determined by two-way ANOVA followed by a Bonferroni comparison test and unpaired Student t-test for AUC, ${ }^{*} P<0.05$ D420 compared to the mock challenged control group, ${ }^{\#} P<0.05$ Tohama 1 compared to the mock challenged control group. 


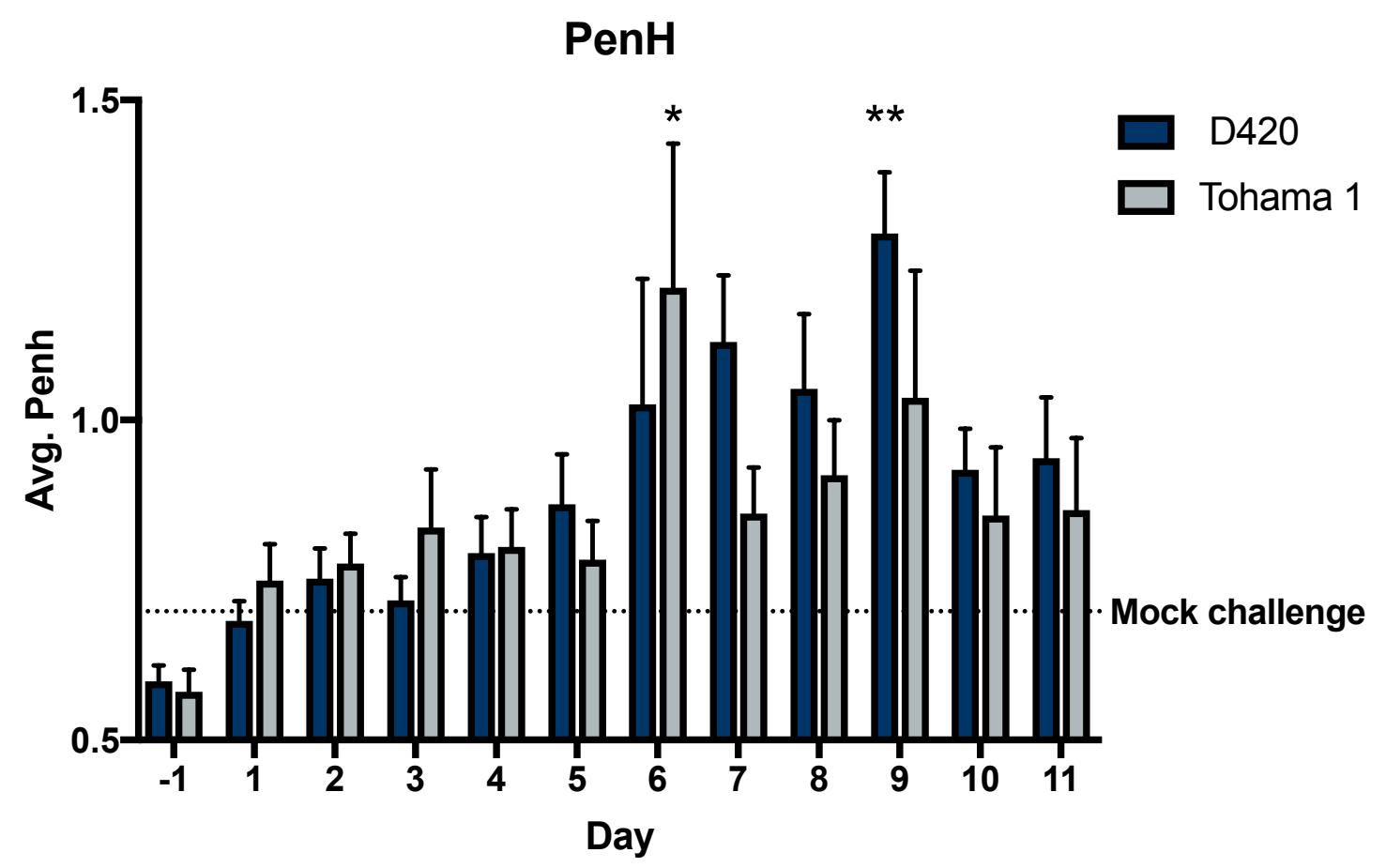

Figure 7. Bordetella pertussis infection impairs respiratory capacity. Over the course of infection, Sprague-Dawley rat's respiratory function was analyzed using whole body plethysmography. Each day at 5p.m., rat's bronchial restriction was determined by utilizing WBP by calculating for PenH. Results shown as mean \pm SEM $(n=4-8)$. P values were determined by two-way ANOVA, ${ }^{*} P<0.05,{ }^{* *} P<0.01$ compared to the mock challenge group followed by Dunnett's comparison test. 
A
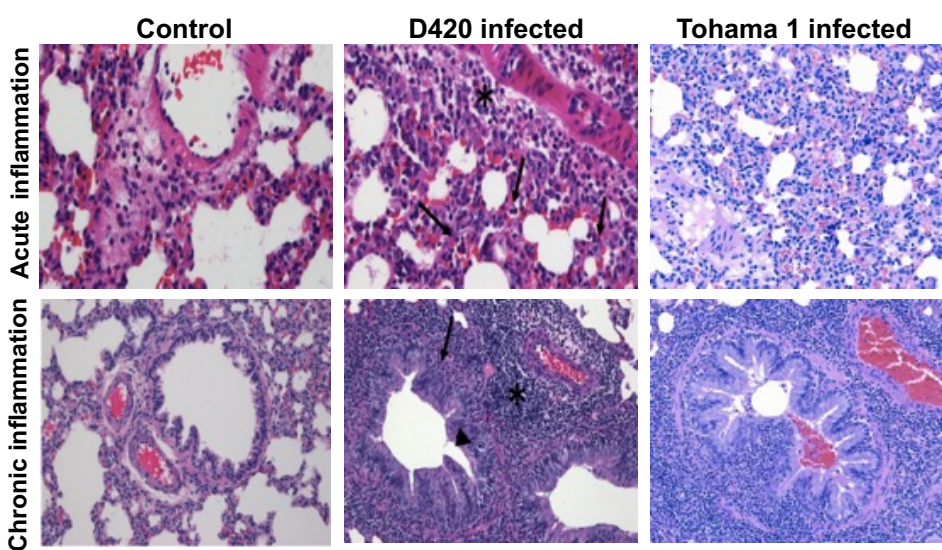

B

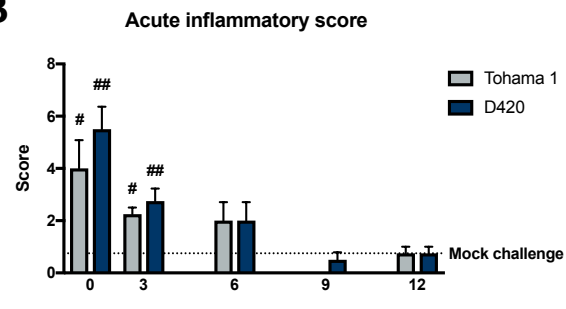

D

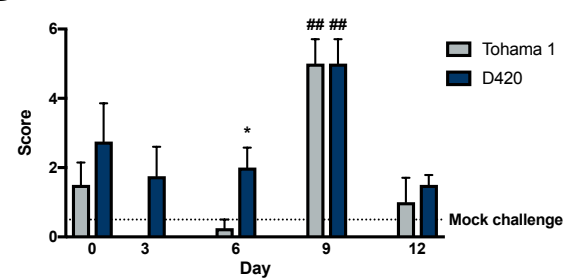

Figure 8. Bp infection induces acute and chronic inflammation in the lung of SpragueDawley rats. The left lobe of the lung was sectioned and stained with hematoxylin and eosin from rats infected with D420, Tohama 1, or the PBS control. (A) Representative images of acute inflammation demonstrating neutrophil recruitment around surrounding blood vessel (asterisk) and parenchyma (arrows) at 400x magnification. (B) Average acute inflammatory score of the lung by the predominance of neutrophils in the parenchyma, blood vessels, and airways. (C) Representative images of chronic inflammation showing mononuclear cells surrounding the blood vessel (asterisk), lamina propria (arrow), and bronchioles (arrowhead) at 200x magnification. (D) Average chronic inflammatory score of the lung characterized by mononuclear infiltrates in the parenchyma, blood vessels, and airway. Histological assessment was determined blinded with no knowledge of the treatment groups. Results are shown as mean \pm SEM $(\mathrm{n}=4) P$ values were determined by one-way ANOVA followed by Dunnett's comparison test, ${ }^{*} P<0.05$ compared between challenge groups. $\# P<0.05$, \#\#P<0.01 compared to mock challenge. 
A

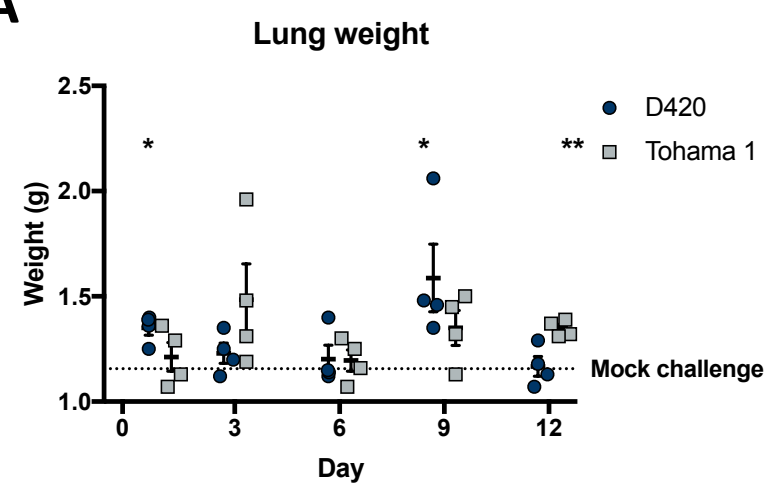

C

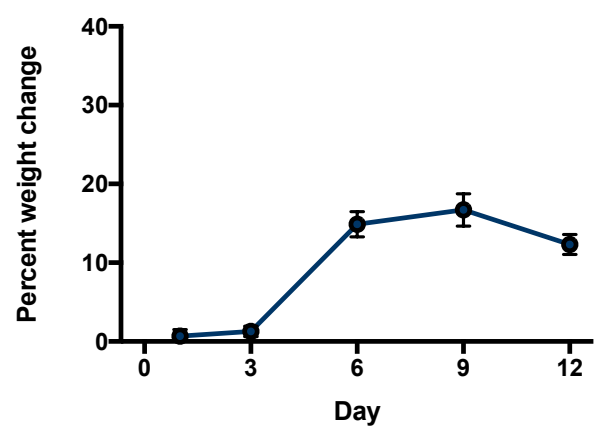

B

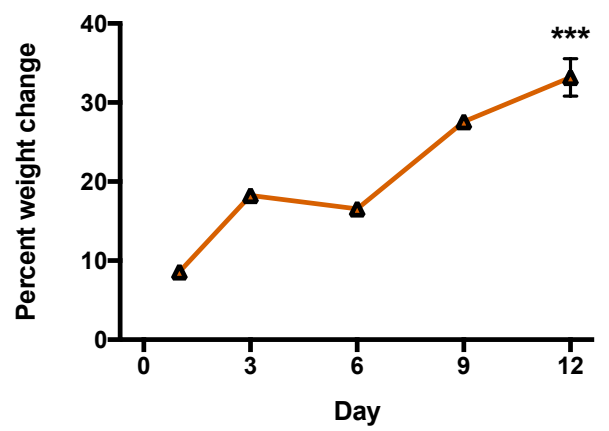

D

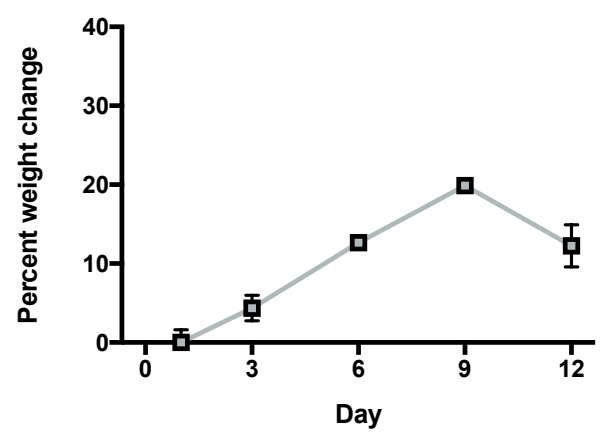

Figure 9. Bp infection induces inflammation of the lung and decrease in weight gain. (A) Post euthanasia, the lung weight was measured and recorded. To observe any weight changes over the course of infection, we calculated percent weight change for (B) mock challenge, (C) D420, (D) Tohama 1 infected rats. Rats body weights were recorded before infection as well as immediately post euthanasia. Percent weight change was calculated by taking the differences between starting weight and end weight and dividing by the initial weight and multiplying by 100 . Results shown as mean \pm SEM $(n=1-4)$. Only one mock challenge rat weight was measured at days $1,3,6$, and $9 . P$ values were determined by one-way ANOVA, ${ }^{*} P<0.05,{ }^{* *} P<0.01,{ }^{* \star *} P<0.001$, compared to the mock challenge group. 
A

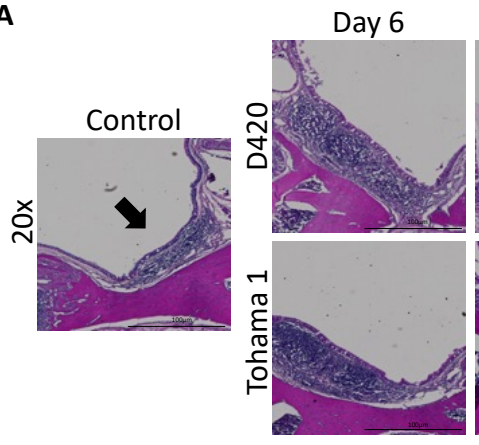

Day 9

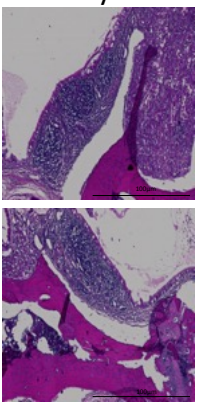

Day 12

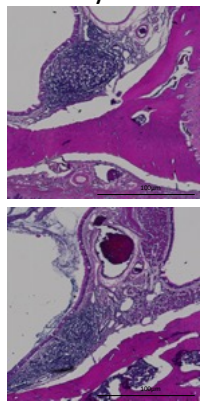

B

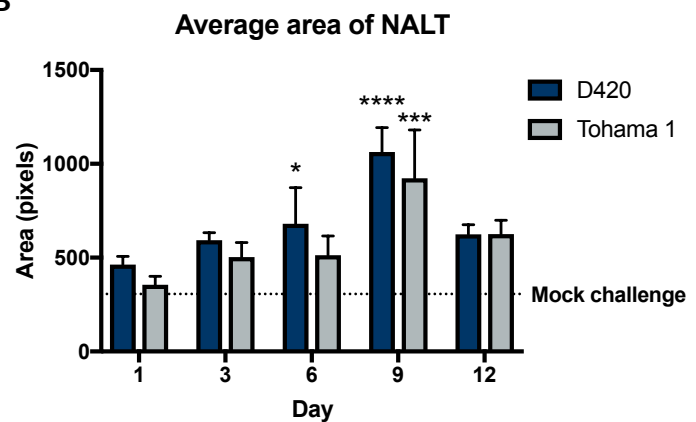

Figure 10. Analysis of the nasal associated lymphoid tissue. The skull was sectioned allowing for staining of the NALT with hematoxylin and eosin. (A) Representative 20x images of the NALT. Arrows indicate the NALT. (B) ImageJ analysis analysis of the area of both the left and right NALT. Dotted line represents the average area of the NALT in the mock challenged group. Results shown as mean $\pm \operatorname{SEM}(n=4)$. $P$ values were determined by two-way ANOVA, ${ }^{*} P<0.05,{ }^{* * *} P<0.001,{ }^{* * * *} P<0.0001$ compared to the mock challenge group. 

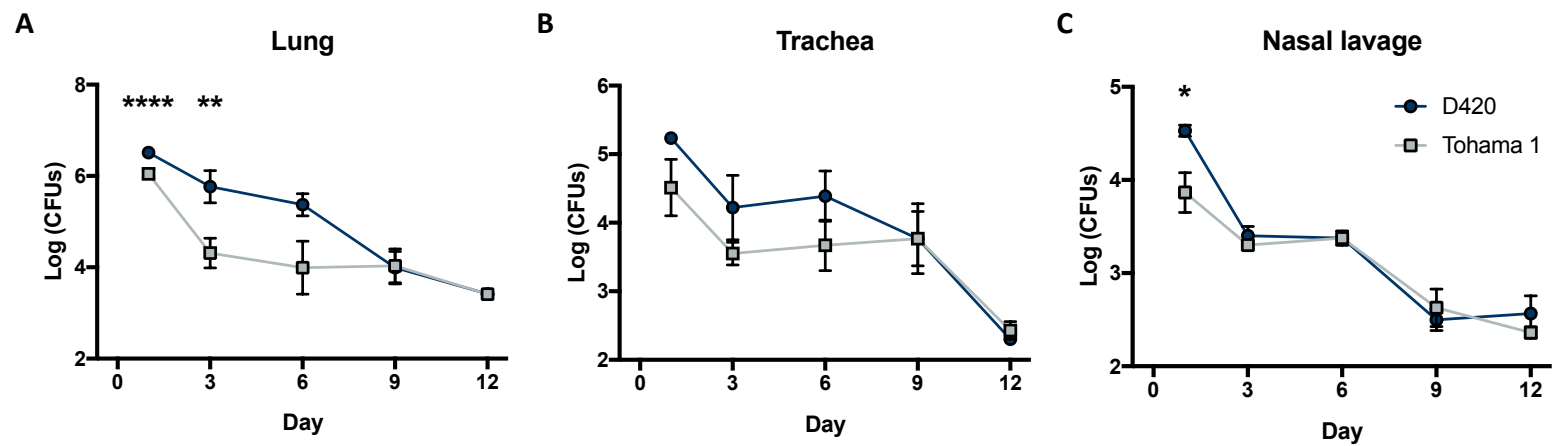

Figure 11. Analysis of bacterial burden over the course of $B p$ infection. Bacteria were quantified by serially diluted CFUs following intranasal challenge. CFU counts were determined from (A) lung homogenate $(B)$ trachea and $(C)$ nasal lavage. Results are shown as mean \pm SEM $(n=4) P$ values were determined by two-way ANOVA corrected with Bonferroni comparison test, ${ }^{*} P<0.05,{ }^{* *} P<0.01,{ }^{* * *} P<0.0001$ compared between D420 and Tohama 1. 


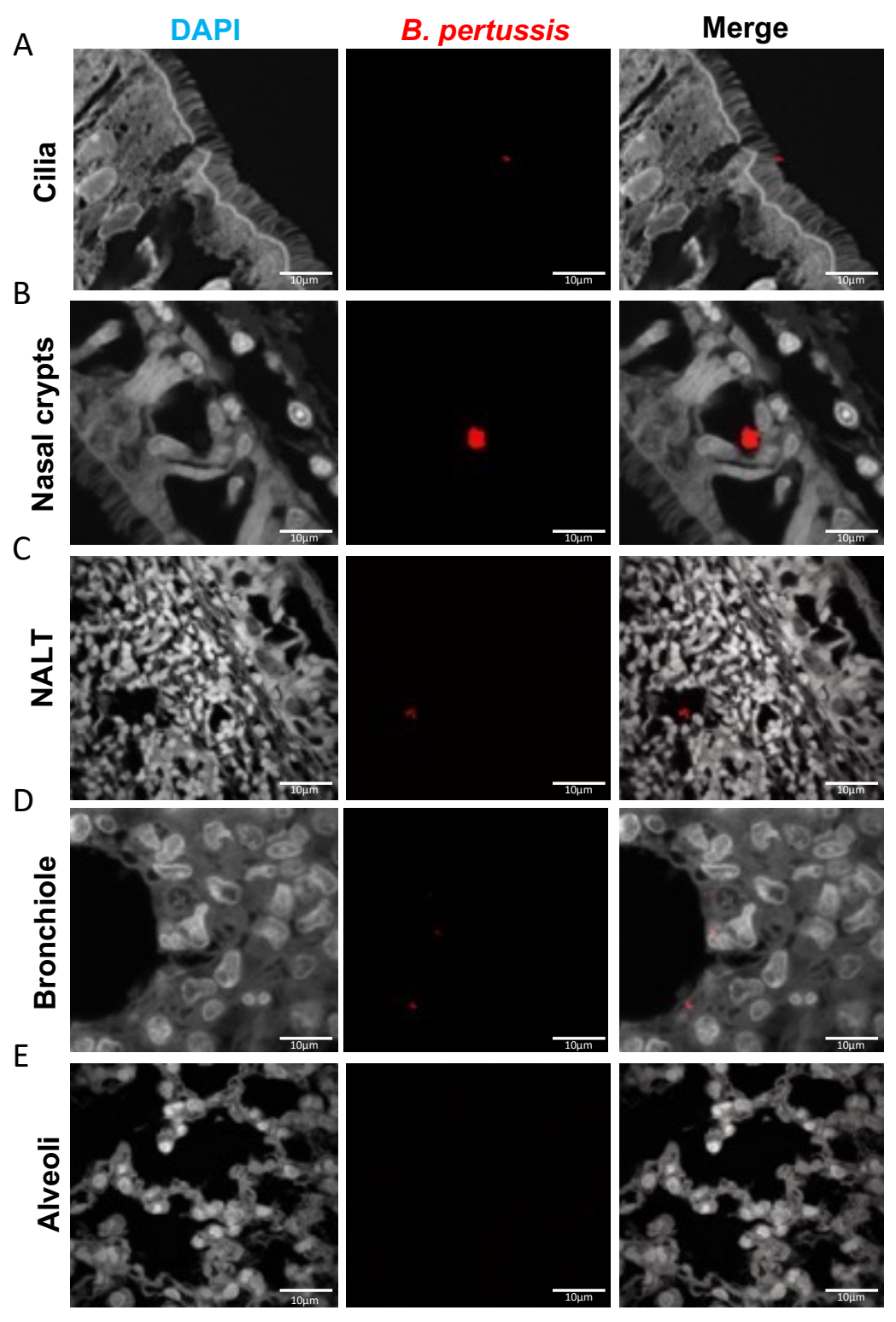

Figure 12. Immunofluorescence (IF) staining of $B p$ localization in the respiratory tract. $B p$ was labeled using a polyclonal antibody to $\mathrm{FHA}$ and counter tagged with a fluorescently conjugated antibody (Texas-Red). Sections were counterstained with DAPI. (A-C) Representative images of $B p$ in the nasal cavity over the course of infection. $B p$ was found captured in the cilia of the nasal cavity as well as the NALT. (D-E) Representative images of the bronchiole and alveoli of infected rats. $B p$ was found localized in the bronchioles over the course of infection and absent in the alveoli. 
A Neutrophils in the blood

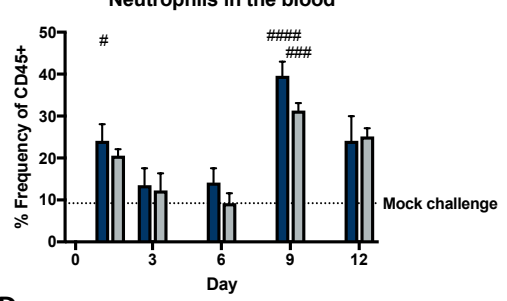

D

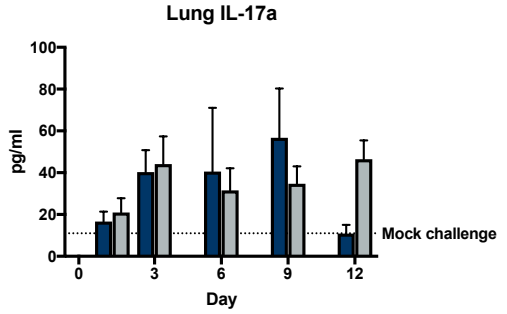

B
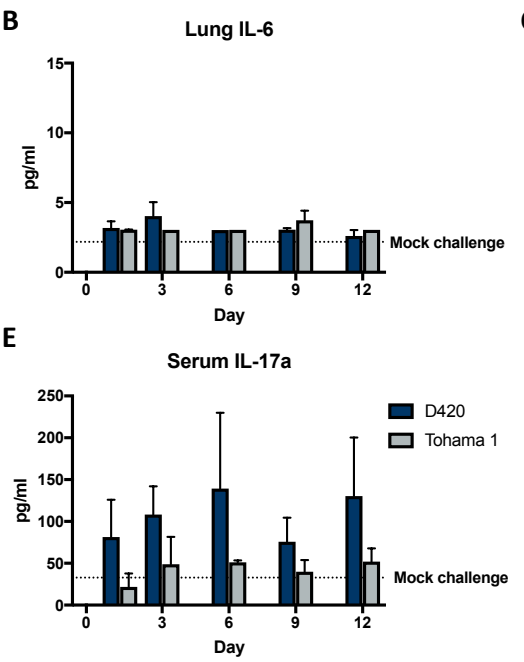

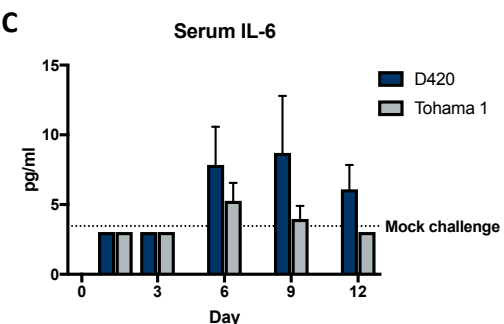

Figure 13. Neutrophil recruitment and proinflammatory cytokine production after challenge determined by flow cytometry and multiplex immunoassay analysis. (A) Blood neutrophil count of Sprague-Dawley rats infected with Bordetella pertussis. At days 1, 3, 6, 9 and 12 post infection, rats were euthanized, and the blood was collected via cardiac puncture. Flow cytometry was used to quantify the amount of neutrophils (CD45 CD161-

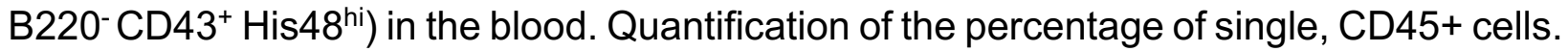
Dotted line represents the average frequency of CD45+ neutrophils in the mock challenge animals over the course of the study. (B-C) Analysis of cytokine IL-6 from supernatant of lung homogenate and serum. (D-E) analysis of cytokine IL-17 in the serum and lung homogenate supernatant. Cytokines analyzed using ProcartaPlex multiplex immunoassay kit. Results shown as mean \pm SEM $(n=4)$. Dotted line represents the average mock challenged cytokine levels measured. $P$ values were determined by oneway ANOVA followed by Tukey comparison test, ${ }^{\#} P<0.05,{ }^{\#} P<0.01,{ }^{\# \# \# P<0.0001}$ compared to mock challenge. 
A

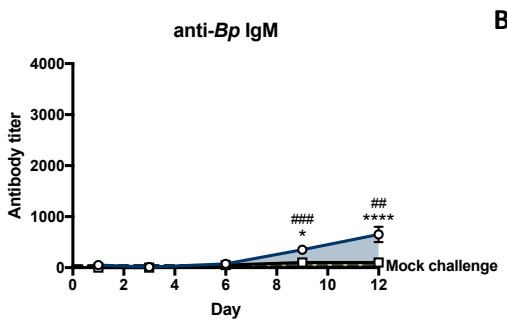

D

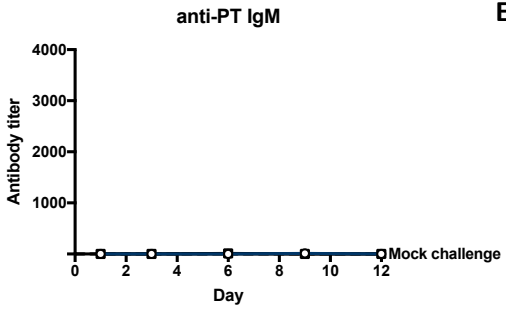

F
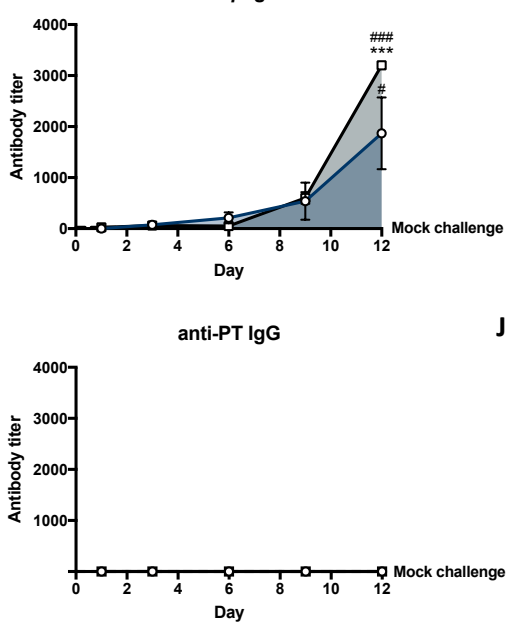

B

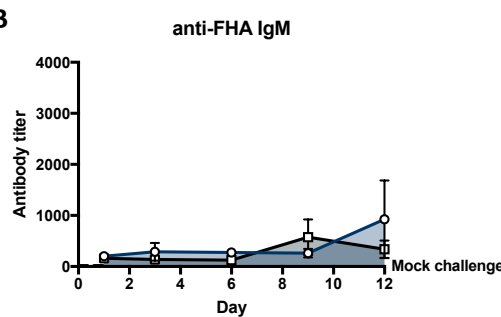

E

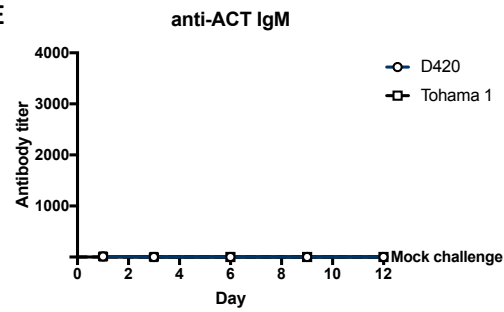

G
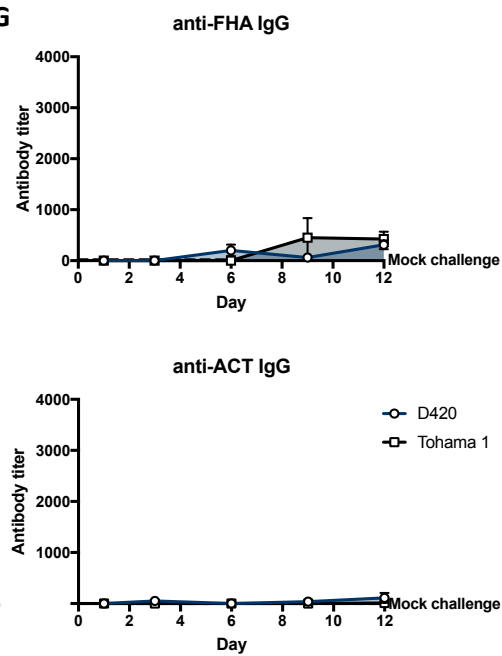

C

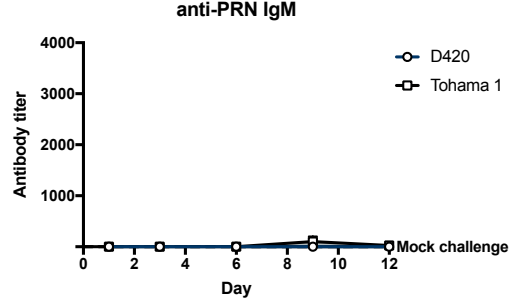

H

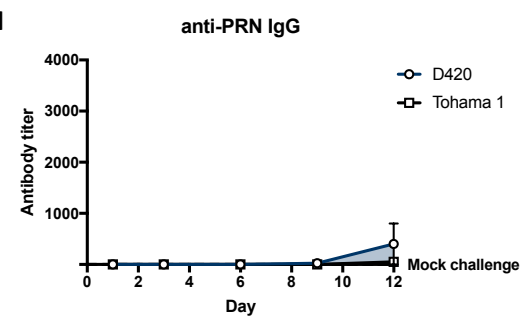

Figure 14. Measurement of serum antibody titers over the course of $B$. pertussis infection. ELISA was used to compare serological responses from rats IN challenge with $B$. pertussis. Total IgM and IgG serum antibody titers were measured from challenged rats against (A \& F) whole B. pertussis bacterium, (B \& G) FHA, (C \& H) PRN, (D \& I) PT, and $(E \&$ J) ACT. Dotted line represents the average serum titer for mock challenge rats. $P$ values were determined by two-way ANOVA corrected with Bonferroni comparison test, ${ }^{*} P<0.05{ }^{* * *} P<0.001,{ }^{* * * *} P<0.0001$ compared between infection groups. For comparison to mock challenged control group one-way ANOVA was used followed by Tukey comparison test, ${ }^{\#} P<0.01,{ }^{\# \#} P<0.001$. 


\subsection{Supplemental Figures}

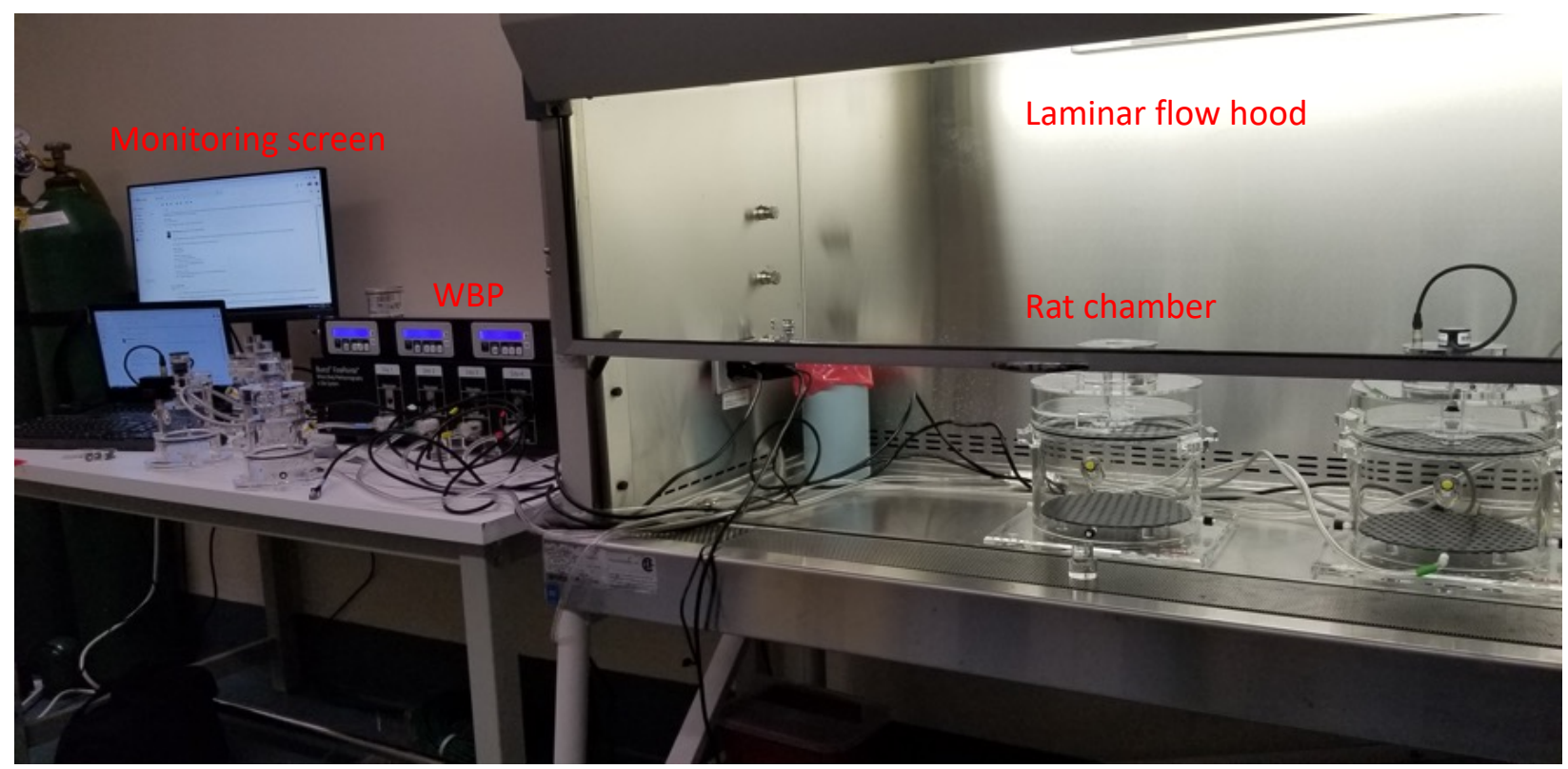

Figure S 1. Set up for the whole-body plethysmograph (WBP). Pictured far left on the table is the WBP and computer. Pictured on the right in the bio safety hood are the chambers where the rats are monitored. 


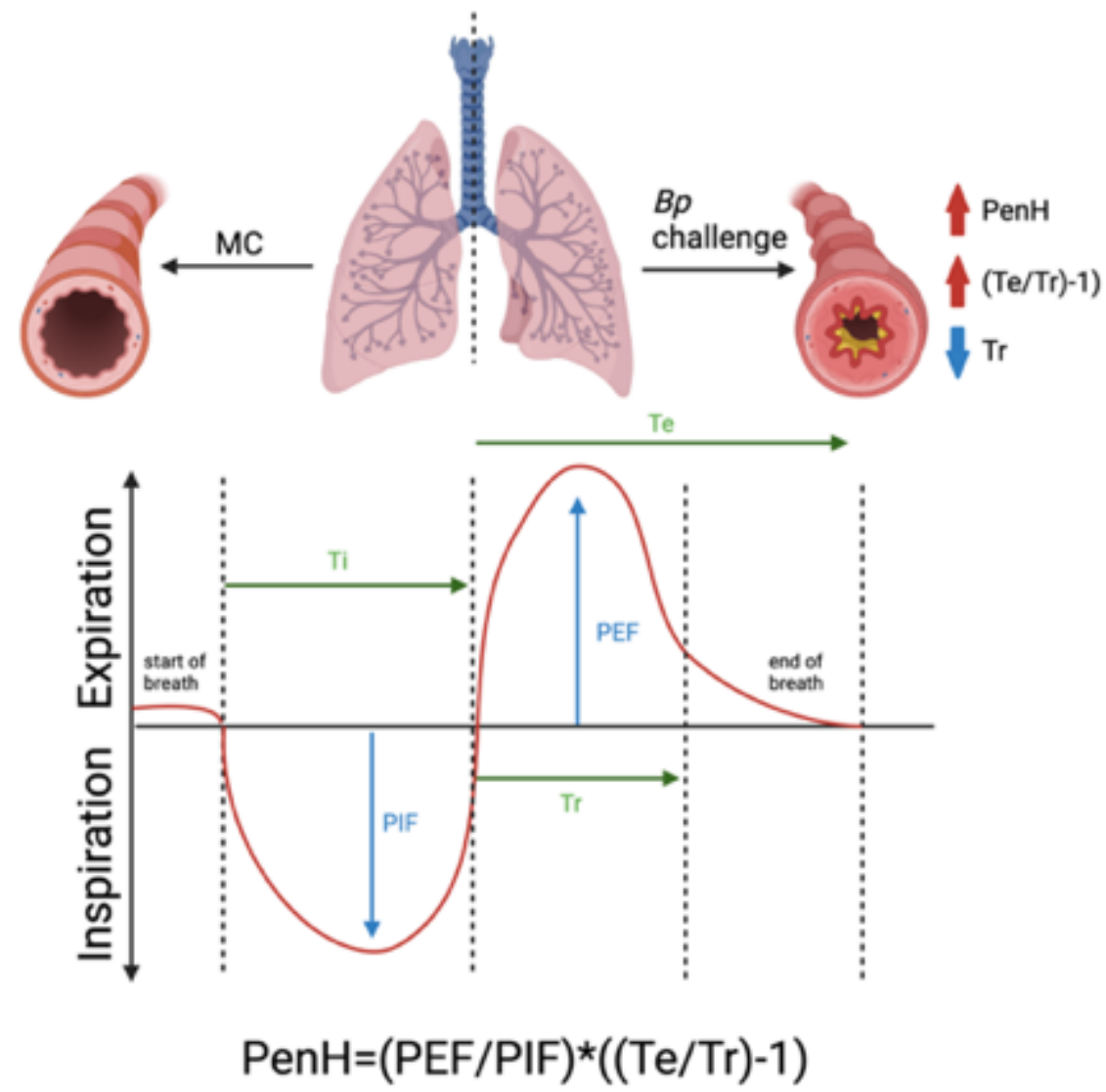

Figure S 2. Diagram illustrating Penh during $B p$ infection. Enhanced pause (Penh) is a calculation that is used as a measurement for respiratory distress. Peak expiratory height (PEF) constitutes as the maximum expiratory flow that occurs in one breath; peak inspiratory flow (PIF) is the maximum inspiratory flow that occurs in one breath; Inspiratory time $\mathrm{(Ti}$ ) the time it takes from the start to end of the inspiration phase of the breath; expiratory time (Te) the amount of time it takes from the start for the expiration to the end of the respiration; relaxation time $(\mathrm{Tr})$ the time it takes the subject to expel certain amount of volume. Following $B p$ challenge, infected rats have an increase in respiratory distress compared to mock challenge rats. 


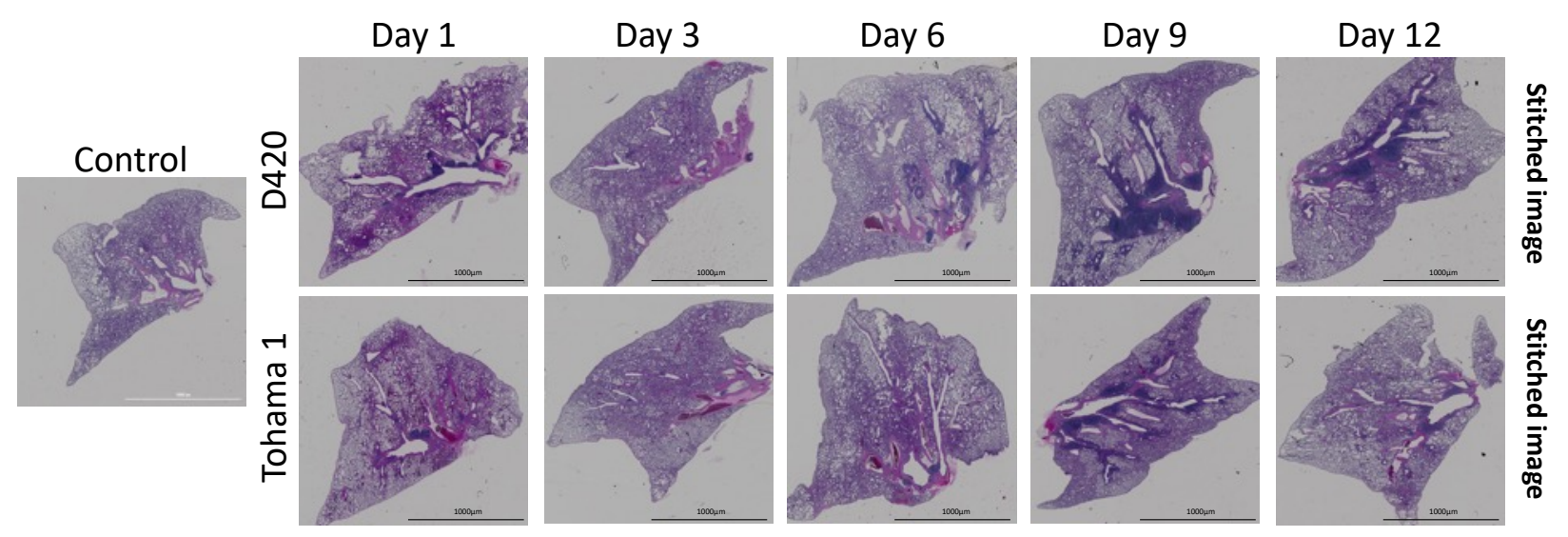

Figure S 3. Bordetella pertussis infection induces cellular recruitment in the lungs. (A) The left lobe of the lung was sectioned and stained with hematoxylin and eosin of rats infected with D420, Tohama 1, or the PBS control. Representative pictures shown are multiple $4 x$ magnification images stitched together for each lung. 


\section{CFUs for each tissue per body weight}

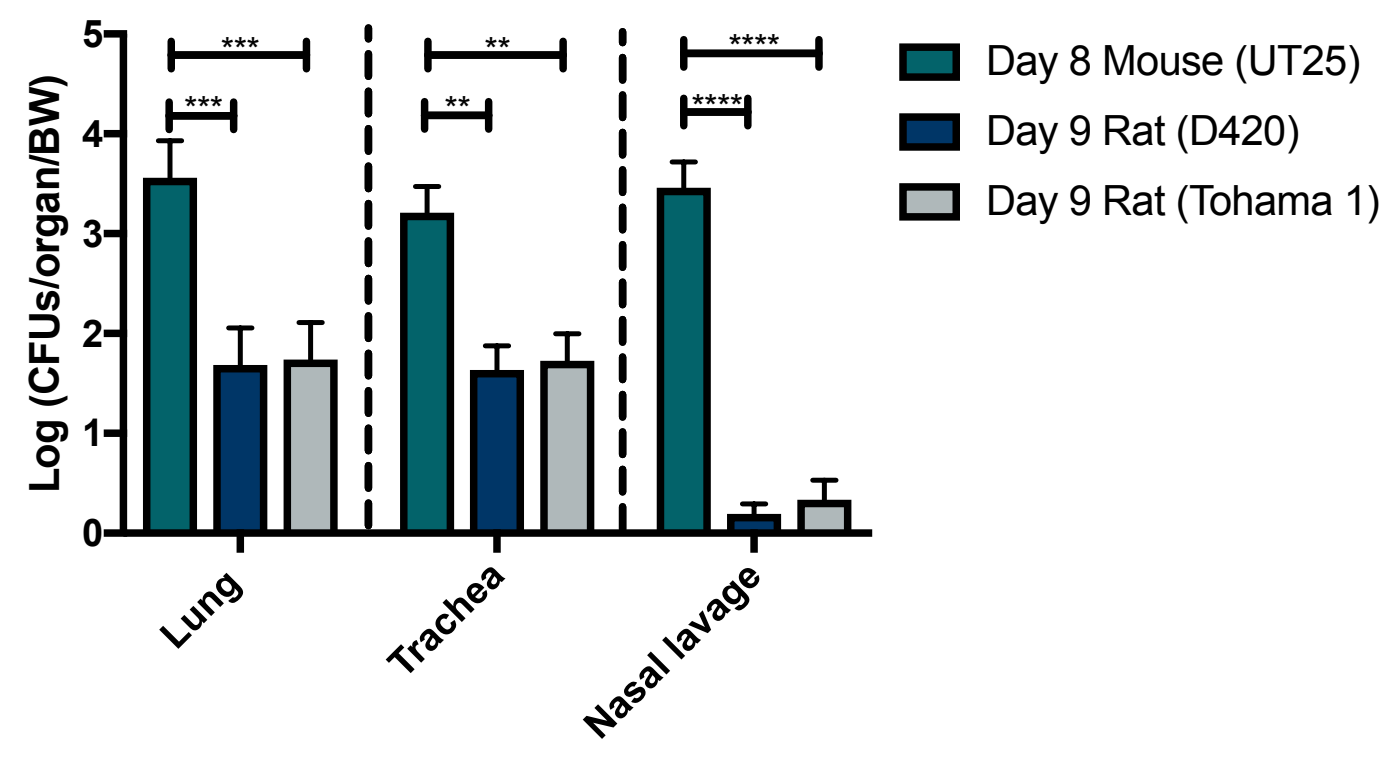

Figure S 4. Bacterial colonization comparisons between CD1 mice and Sprague-Dawley rats. To assess the effect of body weight on bacterial colonization, we took CFUs for each respective tissue and divided them by the body weight. CFUs/organ for the CD1 mice came from (Boehm 2018). Results are shown as mean $\pm \operatorname{SEM}(n=4) P$ values were determined by two-way ANOVA. ${ }^{*} P<0.05,{ }^{* *} P<0.01,{ }^{* * *} P<0.001,{ }^{* * *} P<0.0001$ compared between groups. 


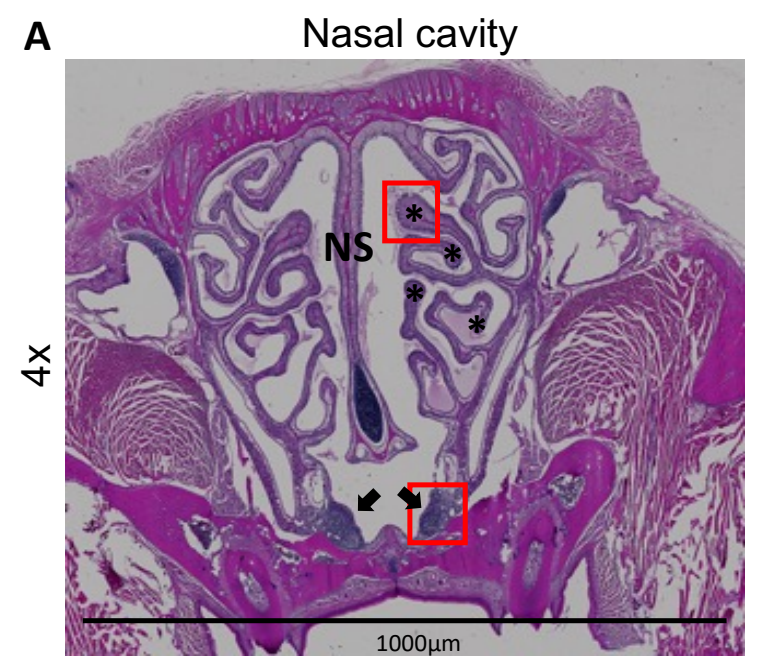

C

Microcolonies in the Bronchioles

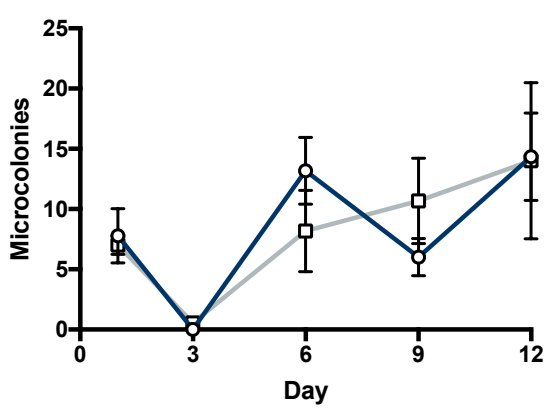

B Nasal turbinate

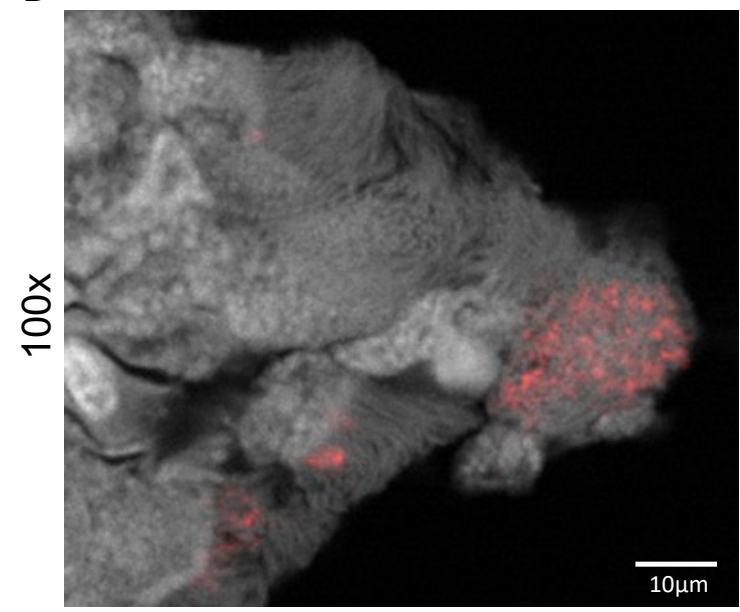

D

Microcolonies in the Nasal Cavity

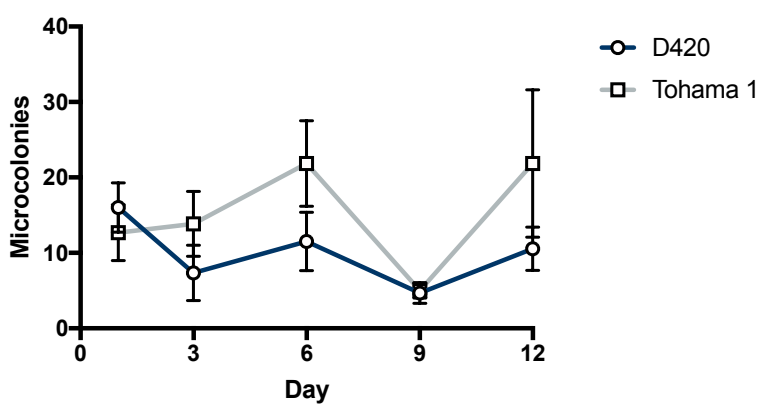

Figure S 5. (A) Hematoxylin and eosin staining of the nasal cavity of Sprague-Dawley rat. Arrows represent the nasal associated lymphoid tissue, NS represents the nasal septum, * represents the nasal turbinates. Red boxes indicate areas where the bacteria were found. (B) Immunohistochemistry (IHC) staining of Bordetella pertussis being captured by the cilia of the nasal cavity. Slides were prepared as described in the Material and Methods over the course of infection. B. pertussis was labeled using a polyclonal antibody to FHA and probed with a fluorescently conjugated antibody (Texas-Red). Sections were counterstained with DAPI. (C-D) Blinded average microcolony counts of $B$. pertussis in the nasal cavity and the lung. Results shown as mean $\pm \operatorname{SEM}(n=3)$. 


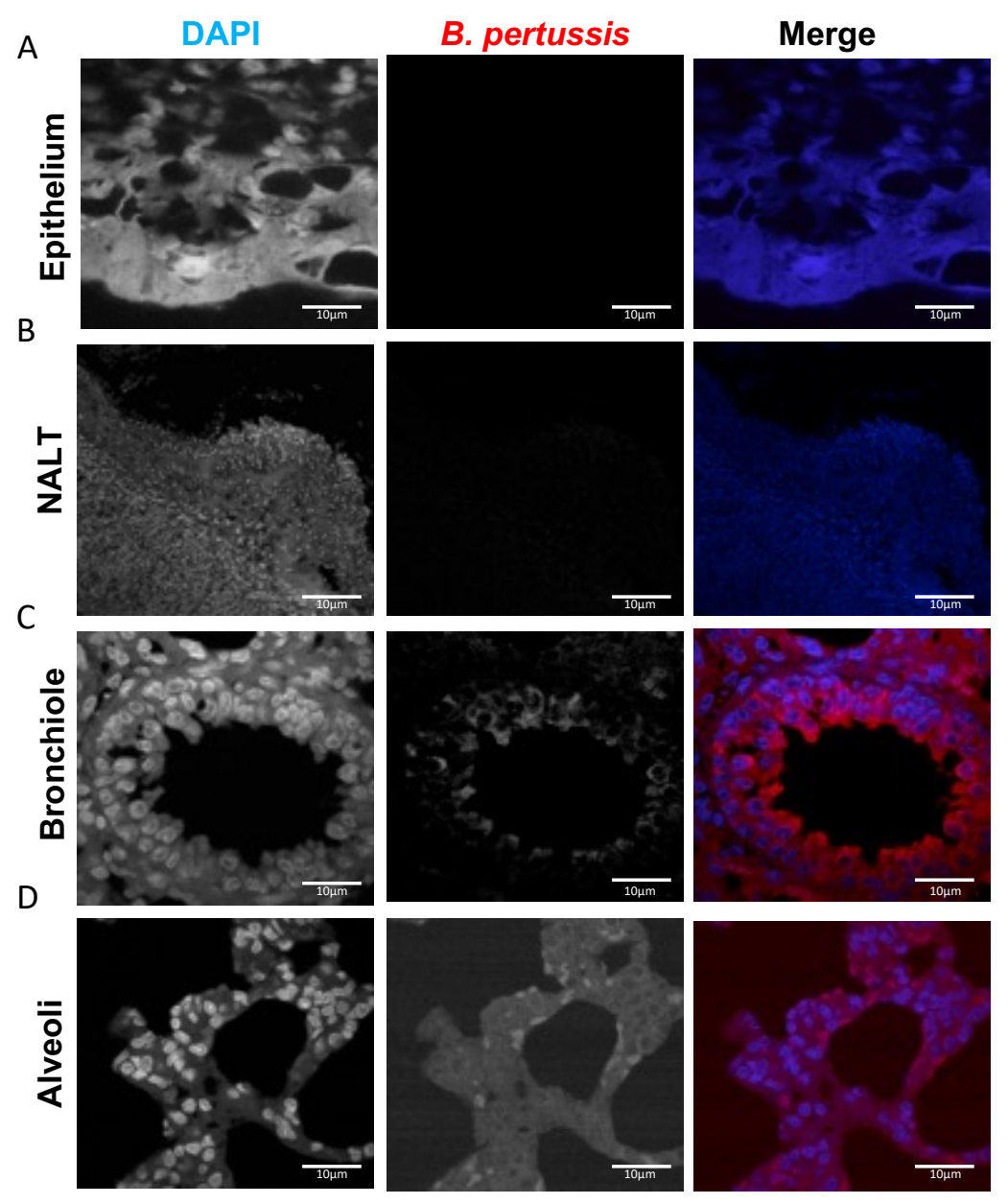

Figure S 6. Immunofluorescence (IF) staining of control rat's respiratory tract. $B p$ was probed for using a polyclonal antibody to FHA and counter tagged with a fluorescently conjugated antibody (Texas-Red). Sections were counterstained with DAPI. (A-D) Representative images of nasal cavity and lung over the course of infection. 

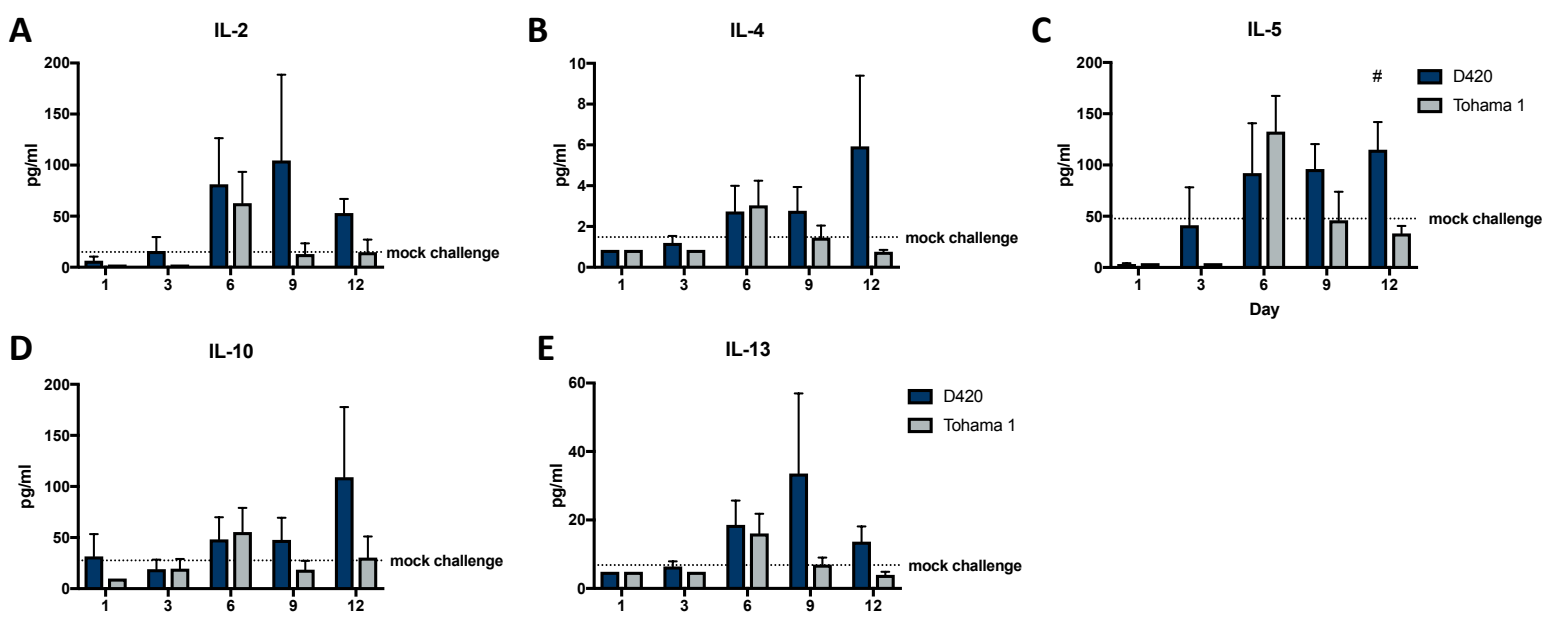

E
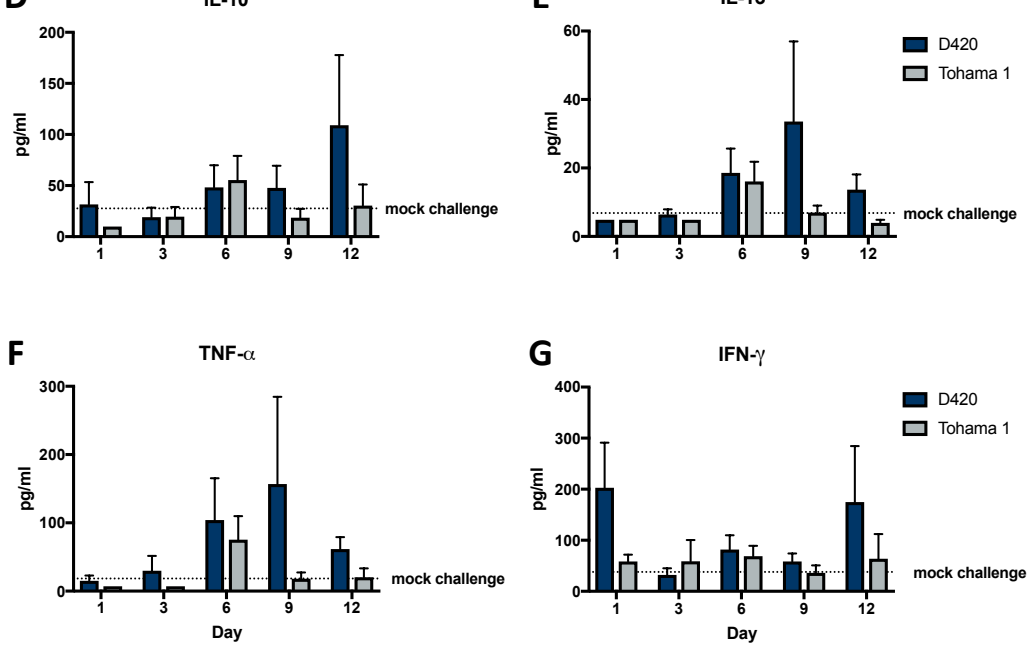

Figure S 7. Serum cytokine response during twelve-day Bordetella pertussis infection. Cytokines measured include: (A) IL-2, (B) IL-4, (C) IL-5, (D) IL-10 (E) IL-13, (F) TNF- $\alpha$, and (G) IFN- $\gamma$. Dotted line represents the average cytokine concentration of the mock challenge control group. Results shown as mean $\pm \operatorname{SEM}(n=4)$. $P$ values were determined by one-way ANOVA followed by Tukey comparison test, $\# P<0.05$ compared between infection groups. 

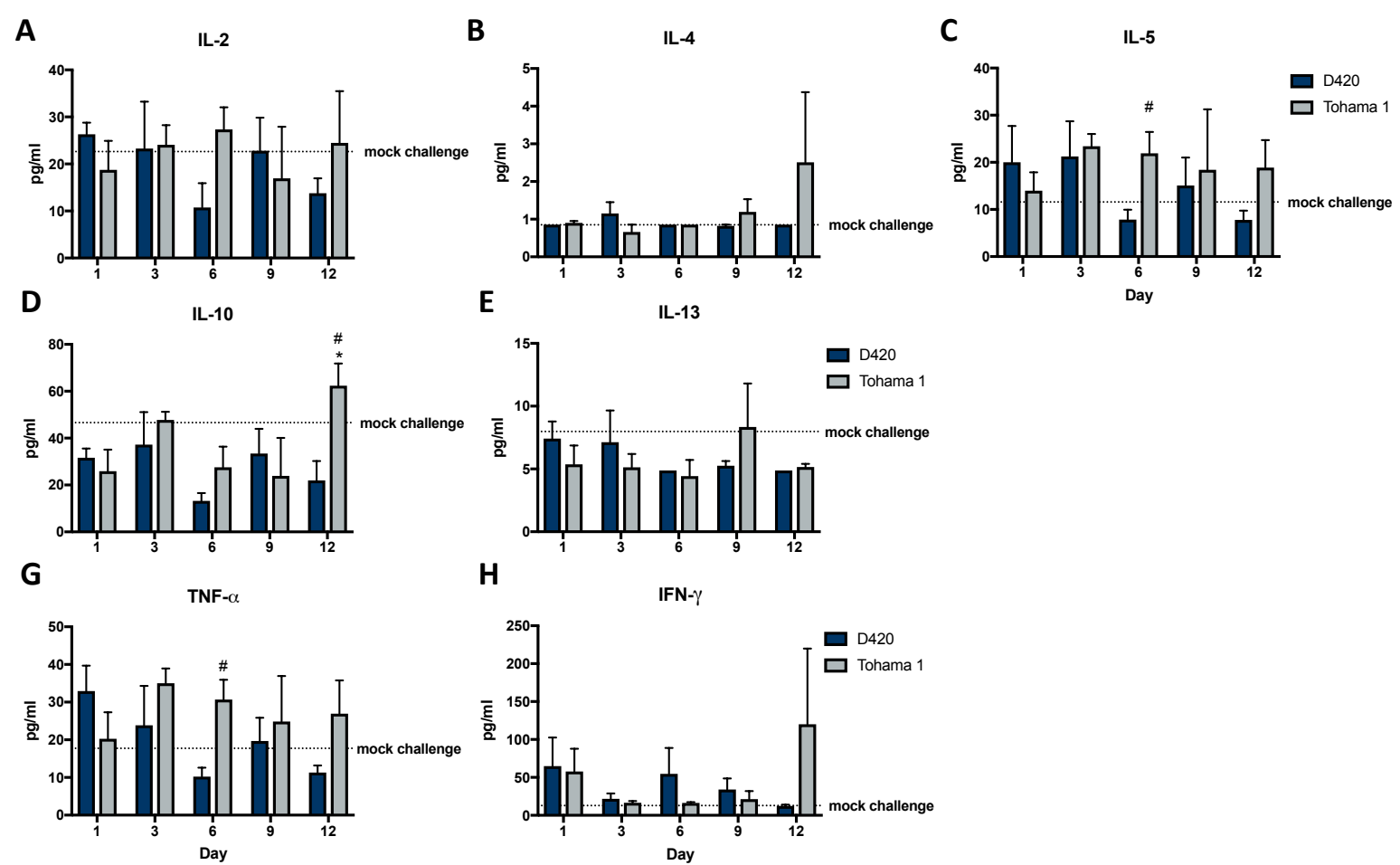

Figure S 8. Lung cytokine response during twelve-day Bordetella pertussis infection. Cytokines measured include: (A) IL-2, (B) IL-4, (C) IL-5, (D) IL-10 (E) IL-13, (F) TNF- $\alpha$, and (G) IFN- $\gamma$. Dotted line represents the average cytokine concentration of the mock challenge control group. Results shown as mean \pm SEM $(n=4)$. $P$ values were determined by one-way ANOVA followed by Tukey comparison test, $\# P<0.05$ compared between infection groups. ${ }^{*} P<0.05$ compared to mock challenge. 

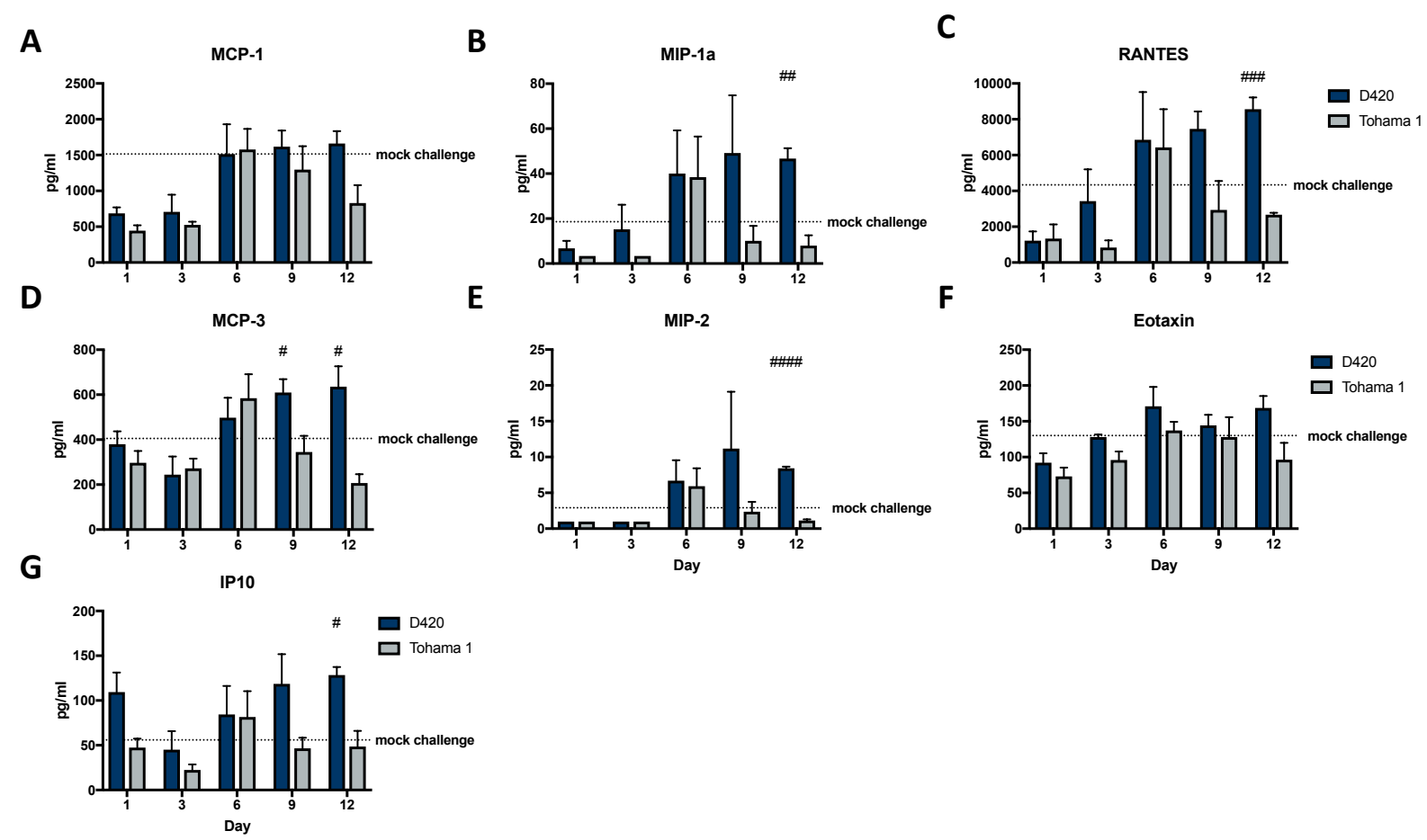

Figure S 9. Serum chemokine during twelve-day Bordetella pertussis infection. Chemokines measured include: (A) MCP-1, (B) MIP-1a, (C) RANTES, (D) MCP-3, (E) MIP-2, (F) Eotaxin, and (G) IP10. Dotted line represents the average cytokine concentration of the mock challenge control group. Results shown as mean \pm SEM ( $n=$ 4). $P$ values were determined by one-way ANOVA followed by Tukey comparison test. $\# P<0.05, \# P<0.01, \# \# P<0.001$ compared between infection groups. 

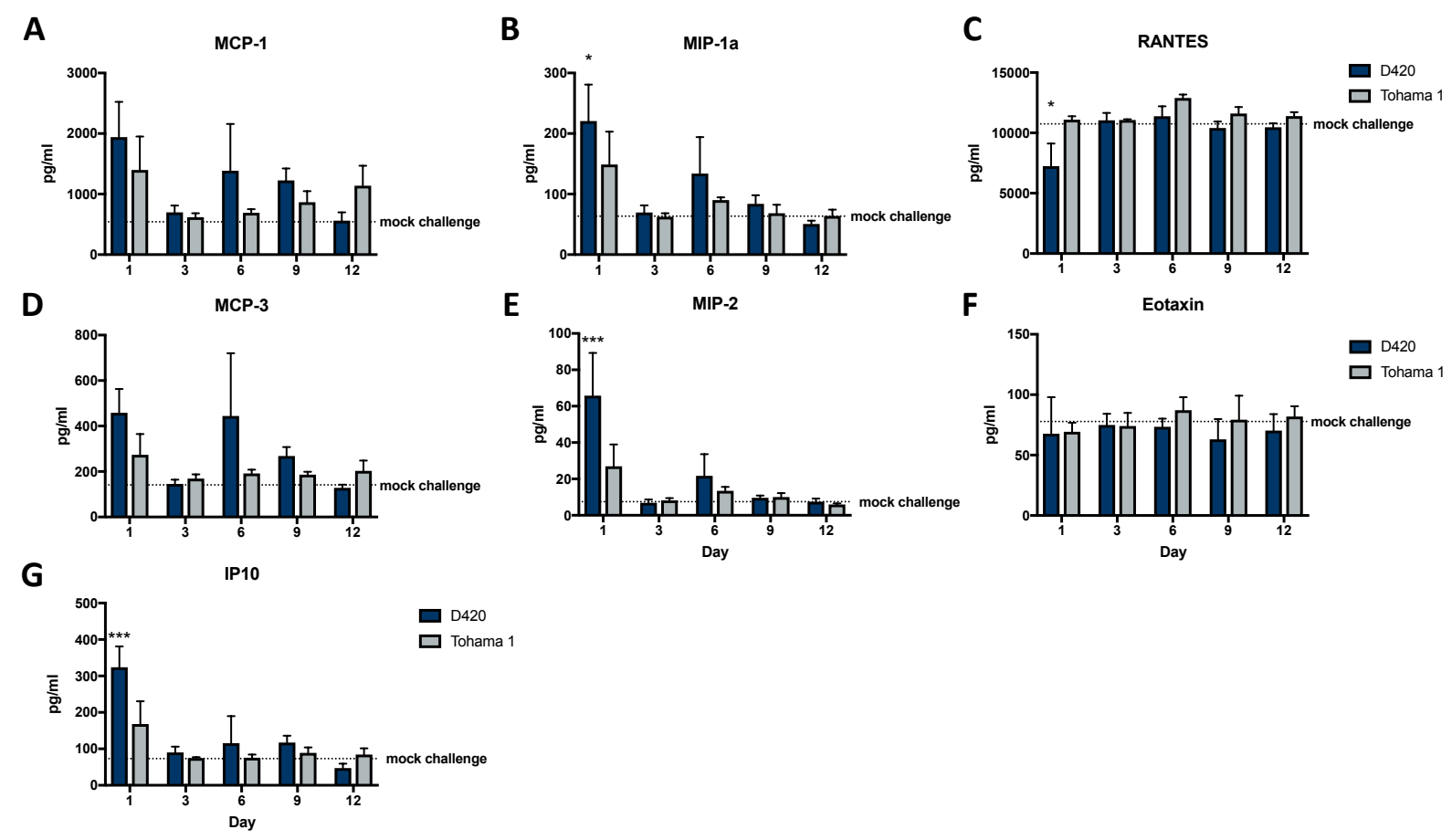

Figure S 10. Lung chemokine during twelve-day Bordetella pertussis infection. Chemokines measured include: (A) MCP-1, (B) MIP-1a, (C) RANTES, (D) MCP-3, (E) MIP-2, (F) Eotaxin, and (G) IP10. Dotted line represents the average cytokine concentration of the mock challenge control group. Results shown as mean \pm SEM $(n=4)$. $P$ values were determined by one-way ANOVA followed by Tukey comparison test. ${ }^{*} P<0.05,{ }^{* * *} P<0.001$ compared to mock challenge. 
A

D420 IgM

Day 12

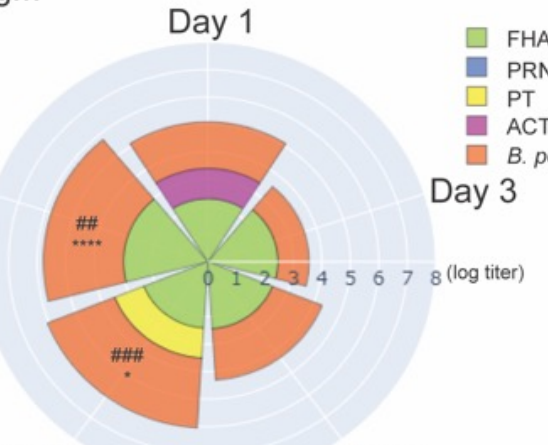

Day 9

Day 6

B

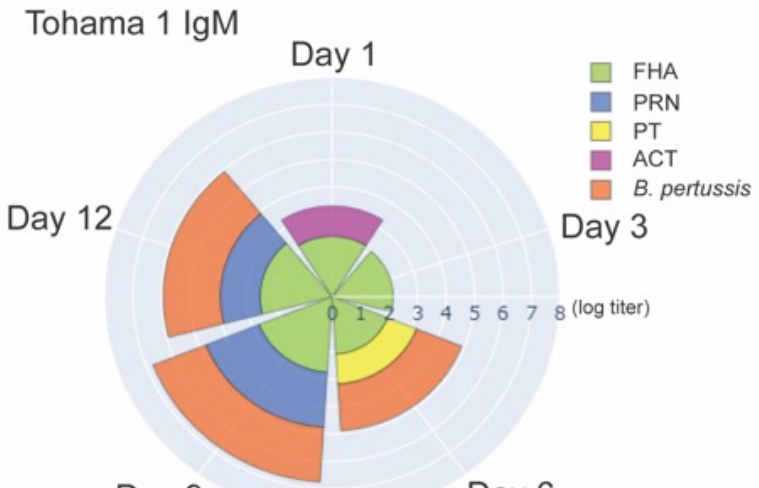

Day 9

Day 6

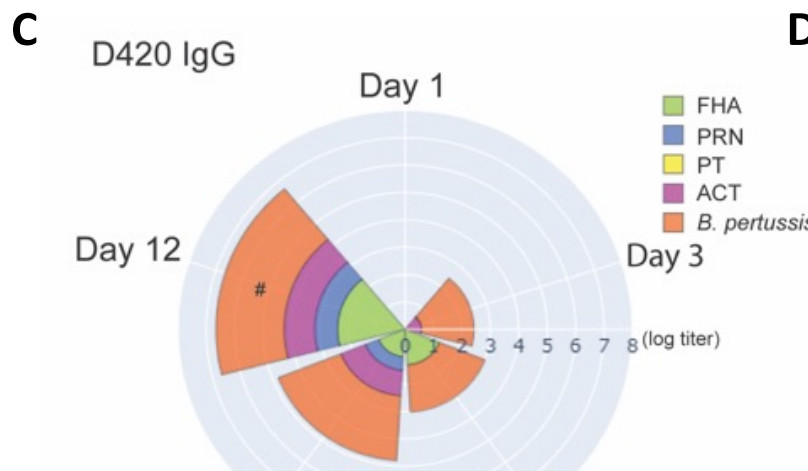

Day 9
Day 6

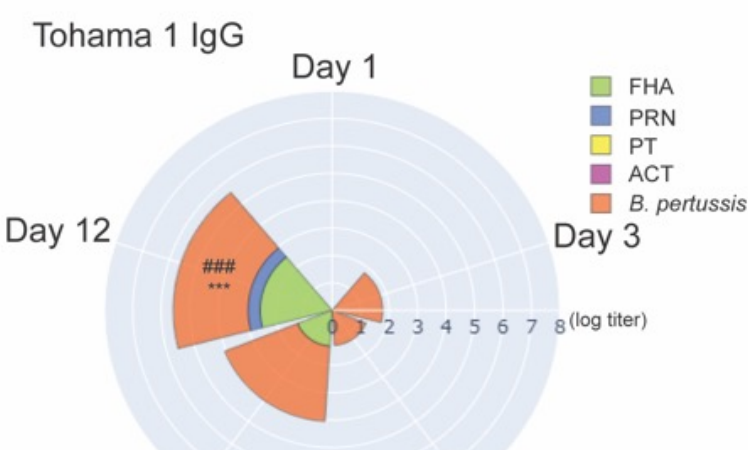

Day 9

Figure S 11. Measurement of serum antibody titers over the course of $B$. pertussis infection. ELISA was used to compare serological responses from rats IN challenge with $B$. pertussis. Total IgM (A-B) and IgG (C-D) serum antibody titers were measured from challenged rats against $B p, \mathrm{FHA}, \mathrm{PRN}, \mathrm{PT}$, and ACT. $(n=4)$. $P$ values were determined by two-way ANOVA corrected with Bonferroni comparison test, ${ }^{*} P<0.05$, ${ }^{* * *} P<0.001$, ${ }^{* * * *} P<0.0001$ compared between infection groups. For comparison to mock challenged control group one-way ANOVA was used followed by Tukey comparison test, ${ }^{\#} P<0.05$, ${ }^{\#} P<0.01,{ }^{\# \#} P<0.001$. 
A anti-Bp lgM

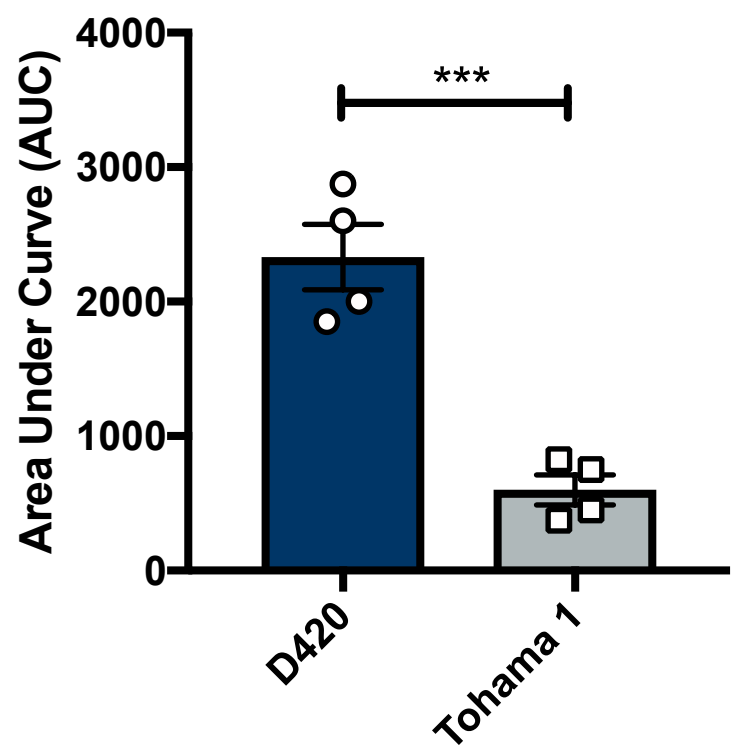

B anti-Bp lgG

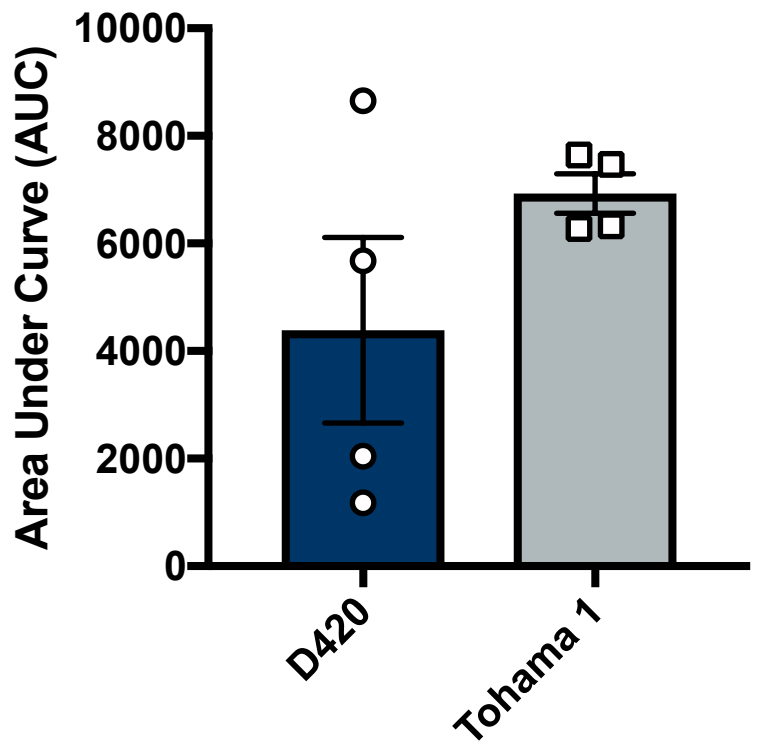

Figure S 12. Area under the curve analysis of (A) IgM and (B) IgG serum antibody titers against Bordetella pertussis over the course of infection. Results shown as mean \pm SEM $(n=4)$. $P$ values were determined by t-test for area under the curve, ${ }^{* * *} P<0.001$ compared between infection groups. 


\subsection{References}

1. Mattoo S, Cherry JD. 2005. Molecular pathogenesis, epidemiology, and clinical manifestations of respiratory infections due to Bordetella pertussis and other Bordetella subspecies. Clin Microbiol Rev 18:326-382.

2. Melvin JA, Scheller E V., Miller JF, Cotter PA. 2014. Bordetella pertussis pathogenesis: Current and future challenges. Nat Rev Microbiol2014/03/13. $12: 274-288$.

3. Weigand MR, Peng Y, Loparev V, Batra D, Bowden KE, Burroughs M, Cassiday PK, Davis JK, Johnson T, Juieng P, Knipe K, Mathis MH, Pruitt AM, Rowe L, Sheth M, Tondella ML, Williams MM. 2017. The History of Bordetella pertussis Genome Evolution Includes Structural Rearrangement. J Bacteriol 199:e00806-16.

4. Elahi S, Holmstrom J, Gerdts V. 2007. The benefits of using diverse animal models for studying pertussis. Trends Microbiol. Trends Microbiol.

5. Cherry JD. 2015. The History of Pertussis (Whooping Cough); 1906-2015: Facts, Myths, and Misconceptions. Curr Epidemiol Reports 2:120-130.

6. Hornibrook JW, Ashburn LL. 1939. A Study of Experimental Pertussis in the Young Rat. Public Heal Reports 54:439.

7. Woods DE, Franklin R, Cryz SJ, Ganss M, Peppler M, Ewanowich C. 1989. Development of a rat model for respiratory infection with Bordetella pertussis. Infect Immun 57:1018-1024.

8. Hall E, Parton R, Wardlaw AC. 1994. Cough production, leucocytosis and serology of rats infected intrabronchially with Bordetella pertussis. J Med Microbiol 40:205- 
213.

9. Parton R, Hall E, Wardlaw AC. 1994. Responses to Bordetella pertussis mutant strains and to vaccination in the coughing rat model of pertussis. J Med Microbiol 40:307-312.

10. Hall E, Parton R, Wardlaw AC. 1997. Differences in coughing and other responses to intrabronchial infection with Bordetella pertussis among strains of rats. Infect Immun 65:4711-4717.

11. Hall E, Parton R, Wardlaw AC. 1998. Responses to acellular pertussis vaccines and component antigens in a coughing-rat model of pertussis. Vaccine 16:1595603.

12. Nakamura K, Shinoda N, Hiramatsu Y, Ohnishi S, Kamitani S, Ogura Y, Hayashi T, Horiguchi Y. 2019. BspR/BtrA, an Anti- $\sigma$ Factor, Regulates the Ability of Bordetella bronchiseptica To Cause Cough in Rats. mSphere https://doi.org/10.1128/msphere.00093-19.

13. Libster R, Edwards KM. 2012. Re-emergence of pertussis: what are the solutions? Expert Rev Vaccines 11:1331-1346.

14. Hill HA, Elam-Evans LD, Yankey D, Singleton JA, Dietz V. 2016. Vaccination Coverage Among Children Aged 19-35 Months - United States, 2015. MMWR Morb Mortal Wkly Rep 65:1065-1071.

15. National, State, and Local Area Vaccination Coverage Among Children Aged 1935 Months — United States, 2011.

16. Klein NP, Bartlett J, Fireman B, Baxter R. 2016. Waning Tdap Effectiveness in 
Adolescents. Pediatrics.

17. Klein NP, Bartlett J, Rowhani-Rahbar A, Fireman B, Baxter R. 2012. Waning Protection after Fifth Dose of Acellular Pertussis Vaccine in Children. N Engl J Med 11367:1012-9.

18. Klein NP, Bartlett J, Fireman B, Rowhani-Rahbar A, Baxter R. 2013. Comparative Effectiveness of Acellular Versus Whole-Cell Pertussis Vaccines in Teenagers. Pediatrics 131:e1716-e1722.

19. Sheridan SL, Frith K, Snelling TL, Grimwood K, McIntyre PB, Lambert SB. 2014. Waning vaccine immunity in teenagers primed with whole cell and acellular pertussis vaccine: recent epidemiology. Expert Rev Vaccines 13:1081-1106.

20. Sheridan SL, Ware RS, Grimwood K, Lambert SB. 2012. Number and order of whole cell pertussis vaccines in infancy and disease protection. JAMA - J Am Med Assoc. American Medical Association.

21. Tartof SY, Lewis M, Kenyon C, White K, Osborn A, Liko J, Zell E, Martin S, Messonnier NE, Clark TA, Skoff TH. 2013. Waning Immunity to Pertussis Following 5 Doses of DTaP. Pediatrics2013/03/13. 131:e1047-e1052.

22. Williams MM, Sen KA, Weigand MR, Skoff TH, Cunningham VA, Halse TA, Tondella ML. 2016. Bordetella pertussis strain lacking pertactin and pertussis toxin. Emerg Infect Dis https://doi.org/10.3201/eid2202.151332.

23. Hall E, Parton R, Wardlaw AC. 1999. Time-course of infection and responses in a coughing rat model of pertussis. J Med Microbiol 48:95-98.

24. Parkhill J, Sebaihia M, Preston A, Murphy LD, Thomson N, Harris DE, Holden MTG, 
Churcher CM, Bentley SD, Mungall KL, Cerdeño-Tárraga AM, Temple L, James K, Harris B, Quail MA, Achtman M, Atkin R, Baker S, Basham D, Bason N, Cherevach I, Chillingworth T, Collins M, Cronin A, Davis P, Doggett J, Feltwell T, Goble A, Hamlin N, Hauser H, Holroyd S, Jagels K, Leather S, Moule S, Norberczak H, O’Neil S, Ormond D, Price C, Rabbinowitsch E, Rutter S, Sanders M, Saunders D, Seeger K, Sharp S, Simmonds M, Skelton J, Squares R, Squares S, Stevens K, Unwin L, Whitehead S, Barrell BG, Maskell DJ. 2003. Comparative analysis of the genome sequences of Bordetella pertussis, Bordetella parapertussis and Bordetella bronchiseptica. Nat Genet2003/08/12. 35:32-40.

25. Boinett CJ, Harris SR, Langridge GC, Trainor EA, Merkel TJ, Parkhill J. 2015. Complete Genome Sequence of Bordetella pertussis D420. Genome Announc 3.

26. Warfel JM, Beren J, Kelly VK, Lee G, Merkel TJ. 2012. Nonhuman primate model of pertussis. Infect Immun2012/01/19. 80:1530-1536.

27. Warfel JM, Merkel TJ. 2014. The baboon model of pertussis: Effective use and lessons for pertussis vaccines. Expert Rev Vaccines 13:1241-1252.

28. Pinto M V., Merkel TJ. 2017. Pertussis disease and transmission and host responses: insights from the baboon model of pertussis. J Infect 74:S114-S119.

29. Warfel JM, Zimmerman LI, Merkel TJ. 2014. Acellular pertussis vaccines protect against disease but fail to prevent infection and transmission ina nonhuman primate model. Proc Natl Acad Sci U S A 111:787-792.

30. Warfel JM, Merkel TJ. 2013. Bordetella pertussis infection induces a mucosal IL-17 response and long-lived Th17 and Th1 immune memory cells in nonhuman 
primates. Mucosal Immunol 6:787-796.

31. KASUGA T, NAKASE Y, UKISHIMA K, TAKATSU K. 1954. Studies on Haemophilus pertussis. V. Relation between the phase of bacilli and the progress of the whooping-cough. Kitasato Arch Exp Med 27:57-62.

32. Caro V, Bouchez V, Guiso N. 2008. Is the Sequenced Bordetella pertussis strain Tohama I representative of the species? J Clin Microbiol 46:2125-8.

33. Sato H, Sato Y. 1985. Protective antigens of Bordetella pertussis mouse-protection test against intracerebral and aerosol challenge of $B$. pertussis. Dev Biol Stand 61:461-7.

34. Barbier M, Boehm DT, Sen-Kilic E, Bonnin C, Pinheiro T, Hoffman C, Gray M, Hewlett E, Damron FH. 2017. Modulation of pertussis and adenylate cyclase toxins by sigma factor RpoE in Bordetella pertussis. Infect Immun 85.

35. Paddock CD, Sanden GN, Cherry JD, Gal AA, Langston C, Tatti KM, Wu K, Goldsmith CS, Greer PW, Montague JL, Eliason MT, Holman RC, Guarner J, Shieh W, Zaki SR. 2008. Pathology and Pathogenesis of Fatal Bordetella pertussis Infection in Infants. Clin Infect Dis 47:328-338.

36. Verheijden KAT, Henricks PAJ, Redegeld FA, Garssen J, Folkerts G. 2014. Measurement of airway function using invasive and non-invasive methods in mild and severe models for allergic airway inflammation in mice. Front Pharmacol 5 AUG.

37. Leist SR, Dinnon KH, Schäfer A, Tse L V., Okuda K, Hou YJ, West A, Edwards CE, Sanders W, Fritch EJ, Gully KL, Scobey T, Brown AJ, Sheahan TP, Moorman NJ, 
Boucher RC, Gralinski LE, Montgomery SA, Baric RS. 2020. A Mouse-Adapted SARS-CoV-2 Induces Acute Lung Injury and Mortality in Standard Laboratory Mice. Cell 183:1070-1085.e12.

38. Dinnon KH, Leist SR, Schäfer A, Edwards CE, Martinez DR, Montgomery SA, West A, Yount BL, Hou YJ, Adams LE, Gully KL, Brown AJ, Huang E, Bryant MD, Choong IC, Glenn JS, Gralinski LE, Sheahan TP, Baric RS. 2020. A mouse-adapted model of SARS-CoV-2 to test COVID-19 countermeasures. Nature 586:560-566.

39. Takaki H, Ichimiya S, Matsumoto M, Seya T. 2018. Mucosal Immune Response in Nasal-Associated Lymphoid Tissue upon Intranasal Administration by Adjuvants. J Innate Immun 10:515-521.

40. Asanuma H, Hodson Thompson A, Iwasaki T, Sato $\mathrm{Y}$, Inaba $\mathrm{Y}$, Aizawa C, Kurata T, Tamura S ichi. 1997. Isolation and characterization of mouse nasal-associated lymphoid tissue. J Immunol Methods 202:123-131.

41. Debertin AS, Tschernig T, Tönjes H, Kleemann WJ, Tröger HD, Pabst R. 2003. Nasal-associated lymphoid tissue (NALT): frequency and localization in young children. Clin Exp Immunol 134:503-507.

42. Hirano T, Kodama S, Moriyama M, Kawano T, Suzuki M. 2009. The role of Toll-like receptor 4 in eliciting acquired immune responses against nontypeable Haemophilus influenzae following intranasal immunization with outer membrane protein. Int J Pediatr Otorhinolaryngol 73:1657-1665.

43. Petukhova G, Naikhin A, Chirkova T, Donina S, Korenkov D, Rudenko L. 2009. Comparative studies of local antibody and cellular immune responses to influenza 
infection and vaccination with live attenuated reassortant influenza vaccine (LAIV) utilizing a mouse nasal-associated lymphoid tissue (NALT) separation method. Vaccine 27:2580-2587.

44. Zuercher AW, Coffin SE, Thurnheer MC, Fundova P, Cebra JJ. 2002. NasalAssociated Lymphoid Tissue Is a Mucosal Inductive Site for Virus-Specific Humoral and Cellular Immune Responses. J Immunol 168:1796-1803.

45. Boehm DT, Hall JM, Wong TY, DiVenere A, Sen-Kilic E, Bevere JR, Bradford SD, Blackwood CB, Elkins C, DeRoos KA, Gray MC, Cooper CG, Varney ME, Maynard JA, Hewlett EL, Barbier M, Damron FH. 2018. Evaluation of adenylate cyclase toxoid antigen in acellular pertussis vaccines using a Bordetella pertussis challenge model in mice. Infect Immun IAI.00857-17.

46. Sawal M, Cohen M, Irazuzta JE, Kumar R, Kirton C, Brundler M-A, Evans CA, Wilson JA, Raffeeq P, Azaz A, Rotta AT, Vora A, Vohra A, Abboud P, Mirkin LD, Cooper M, Dishop MK, Graf JM, Petros A, Klonin H. 2009. Fulminant pertussis: A multi-center study with new insights into the clinico-pathological mechanisms. Pediatr Pulmonol 44:970-980.

47. Barnett-Vanes A, Sharrock A, Birrell MA, Rankin S. 2016. A Single 9-Colour Flow Cytometric Method to Characterise Major Leukocyte Populations in the Rat: Validation in a Model of LPS-Induced Pulmonary Inflammation https://doi.org/10.1371/journal.pone.0142520.

48. Boehm DT, Wolf MA, Hall JM, Wong TY, Sen-Kilic E, Basinger HD, Dziadowicz SA, Gutierrez M de la P, Blackwood CB, Bradford SD, Begley KA, Witt WT, Varney ME, 
Barbier M, Damron FH. 2019. Intranasal acellular pertussis vaccine provides mucosal immunity and protects mice from Bordetella pertussis. npj Vaccines 4:112.

49. Zhang X, Goel T, Goodfield LL, Muse SJ, Harvill ET. 2011. Decreased Leukocyte Accumulation and Delayed Bordetella pertussis Clearance in IL-6-/- Mice. J Immunol 186:4895-4904.

50. Wolf MA, Boehm DT, DeJong MA, Wong TY, Sen-Kilic E, Hall JM, Blackwood CB, Weaver KL, Kelly CO, Kisamore CA, Bitzer GJ, Bevere JR, Barbier M, Damron FH. 2020. Intranasal immunization with acellular pertussis vaccines results in long-term immunity to Bordetella pertussis in mice . Infect Immun IAI.00607-20.

51. Andreasen C, Carbonetti NH. 2009. Role of neutrophils in response to Bordetella pertussis infection in mice. Infect Immun2008/12/24. 77:1182-1188.

52. Solans L, Locht C. 2018. The Role of Mucosal Immunity in Pertussis. Front Immunol 9:3068.

53. de Graaf H, Ibrahim M, Hill AR, Gbesemete D, Vaughan AT, Gorringe A, Preston A, Buisman AM, Faust SN, Kester KE, Berbers GAM, Diavatopoulos DA, Read RC. 2019. Controlled Human Infection With Bordetella pertussis Induces Asymptomatic, Immunizing Colonization. Clin Infect Dis https://doi.org/10.1093/cid/ciz840.

54. Wardlaw AC, Hall E, Parton R. 1993. Coughing rat model of pertussis. Biologicals 21:27-29.

55. Kilgore PE, Salim AM, Zervos MJ, Schmitt H-J. 2016. Pertussis: Microbiology, Disease, Treatment, and Prevention. Clin Microbiol Rev 29:449-86. 
56. Rolin O, Smallridge W, Henry M, Goodfield L, Place D, Harvill ET. 2014. Toll-like receptor 4 limits transmission of Bordetella bronchiseptica. PLoS One 9:85229.

57. Connelly CE, Sun Y, Carbonetti NH. 2012. Pertussis toxin exacerbates and prolongs airway inflammatory responses during Bordetella pertussis infection. Infect Immun 80:4317-4332.

58. Carbonetti NH, Artamonova G V, Andreasen C, Dudley E, Mays RM, Worthington ZE V. 2004. Suppression of Serum Antibody Responses by Pertussis Toxin after Respiratory Tract Colonization by Bordetella pertussis and Identification of an Immunodominant Lipoprotein. Infect Immun 72:3350-3358.

59. Shumilla JA, Lacaille V, Hornell TMC, Huang J, Narasimhan S, Relman DA, Mellins ED. 2004. Bordetella pertussis Infection of Primary Human Monocytes Alters HLADR Expression. Infect Immun 72:1450-1462.

60. Martino A, Volpe E, Auricchio G, Colizzi V, Baldini PM. 2006. Influence of Pertussis toxin on CD1a isoform expression in human dendritic cells. J Clin Immunol 26:153159.

61. Weiss AA, Falkow S. 1982. Plasmid Transfer to Bordetella pertussis: Conjugation and Transformation. Downloaded from JOURNAL OF BACTERIOLOGY.

62. Mooi FR, Van Loo IHM, Van Gent M, He Q, Bart MJ, Heuvelman KJ, De Greeff SC, Diavatopoulos D, Teunis P, Nagelkerke N, Mertsola J. 2009. Bordetella pertussis strains with increased toxin production associated with pertussis resurgence. Emerg Infect Dis 15:1206-1213.

63. Advani A, Gustafsson L, Carlsson RM, Donnelly D, Hallander HO. 2007. Clinical 
outcome of pertussis in Sweden: Association with pulsed-field gel electrophoresis profiles and serotype. APMIS 115:736-742.

64. King AJ, Van Der Lee S, Mohangoo A, Van Gent M, Van Der Ark A, Van De Waterbeemd B. Genome-Wide Gene Expression Analysis of Bordetella pertussis Isolates Associated with a Resurgence in Pertussis: Elucidation of Factors Involved in the Increased Fitness of Epidemic Strains https://doi.org/10.1371/journal.pone.0066150.

65. Andreasen C, Powell DA, Carbonetti NH. 2009. Pertussis toxin stimulates IL-17 production in response to Bordetella pertussis infection in mice. PLoS One 4:e7079.

66. Lee GM, Lett S, Schauer S, Lebaron C, Murphy T V, Rusinak D, Lieu TA. 2004. Societal Costs and Morbidity of Pertussis in Adolescents and Adults.

67. Cherry JD. 2016. Pertussis in young infants throughout the world. Clin Infect Dis 63:S119-S122.

68. Hiramatsu Y, Suzuki K, Nishida T, Onoda N, Satoh T, Akira S, Ikawa M, Ikeda H, Kamei J, Derouiche S, Tominaga M, Horiguchi Y. 2020. Lipooligosaccharide, Vag8, and pertussis toxin of Bordetella pertussis cooperatively cause coughing in mice. bioRxiv. bioRxiv.

69. Bordet J, Gengou O. 1906. Le Microbe de la Coqueluche. Les Ann I'Institut Pasteur $20: 731-741$.

70. Stainer DW, Scholte MJ. 1970. A Simple Chemically Defined Medium for the Production of Phase I Bordetella pertussis. J Gen Microbiol1970/10/01. 63:211220. 
71. Lomask J, Larson R. 2006. United States Patent USOO7104962B2. US 7,104,962

B2. 
Chapter 3. Mucosal immunization with DTaP confers protection against Bordetella pertussis

infection and cough in SpragueDawley rats 
Mucosal immunization with DTaP confers protection against Bordetella pertussis infection and cough in Sprague-Dawley rats

Jesse M. Hall ${ }^{1,2}$, Graham J. Bitzer ${ }^{1,2}$, Megan A. DeJong ${ }^{1,2}$, Jason Kang ${ }^{1,2}$, Ting Y. Wong $^{1,2}$, M. Allison Wolf ${ }^{1,2}$, Justin R Bevere ${ }^{1,2}$ Mariette Barbier $^{1,2}$, \& F. Heath Damron ${ }^{1,2}$

${ }^{1}$ Department of Microbiology, Immunology and Cell Biology, School of Medicine, West Virginia University, Morgantown, West Virginia, USA

${ }^{2}$ Vaccine Development Center, West Virginia University Health Sciences Center, Morgantown, West Virginia, USA

Running title: Mucosal immunization against Bordetella pertussis in rats \#Address correspondence to F. Heath Damron, fdamron@hsc.wvu.edu 


\subsection{ABSTRACT}

Pertussis is a respiratory disease caused by the Gram-negative pathogen, Bordetella pertussis $(B p)$. The transition from a whole cell pertussis vaccine (wP; DTP) to an acellular pertussis vaccine (aP; DTaP; Tdap) correlates with an increase in pertussis cases, despite widespread vaccine implementation and coverage, and it is now appreciated that the protection provided by aP rapidly wanes. To recapitulate the localized immunity observed from natural infection, mucosal vaccination with aP was explored using the coughing rat model of pertussis. Immunity induced by both oral gavage (OG) and intranasal (IN) vaccination of aP in Bp challenged rats over a nine-day infection was compared to intramuscular (IM)-wP and IM-aP immunized rats that were used as positive controls as IM immunization is the current route for wP and aP vaccination. Our data demonstrate that both IN and OG immunization of aP resulted in production of anti$B p \lg$ antibody titers similar to IM-wP and IM-aP vaccinated controls post-challenge. INaP also induced anti-Bp IgA antibodies in the nasal cavity. Immunization with IM-wP, IMaP, IN-aP, and OG-aP immunization protected against $B p$ induced cough, while OG-aP immunization did not protect against respiratory distress. Mucosal immunization (IN-aP and OG-aP) also protected against acute inflammation and decreased bacterial burden in the lung compared to mock vaccinated challenge (MVC) rats. The data presented in this study suggests that mucosal vaccination with aP can induce a mucosal immune response and provide protection against $B p$ challenge. 


\subsection{Introduction}

Infection of the respiratory mucosa by the Gram-negative bacterium Bordetella pertussis $(B p)$ causes the disease known as pertussis (whooping cough) (1). Clinical manifestations of pertussis are characterized by paroxysmal cough, hypertension, leukocytosis and in severe cases death, particularly in infants who have yet to receive their first vaccine dose (2-4). Before pertussis vaccines were introduced in the United States, pertussis led to approximately 200,000 deaths annually (5). Largely, this disease has been under control by the use of diphtheria tetanus whole-cell pertussis (DTP; wP) and acellular pertussis (DTaP; Tdap; aP) vaccines. DTP was first introduced in the 1940s/1950s, and was largely effective in decreasing pertussis incidence (6). Due to the robust immune response and reactogenicity concerns, developed countries converted to the use of DTaP in the 1990s (2).

Since the introduction of the aP vaccine, pertussis cases have been increasing, despite high vaccine coverage. It has been hypothesized that the increase in pertussis cases is attributed to: waning immunity from DTaP and Tdap vaccination, vaccine driven evolution of $B p$ strains, increased surveillance of pertussis, increased asymptomatic transmission, and improved PCR based molecular identification of cases (7-13). Infant baboons vaccinated with DTaP (1 human dose at 2, 4, and 6 months of age) were still colonized after experimental $B p$ challenge in addition to subsequent transmission to naïve baboons (14). In the same study, Warfel et al (2014) showed that convalescent baboons that cleared a prior $B p$ infection, were not colonized following re-challenge of $B p$ one month 
later (14). In humans, studies suggest that convalescent immunity can confer protection for approximately 4-20 years (15), while DTaP immunity falls short lasting on average 412 years (15). Overall, these data demonstrated that immunity induced through natural infection can generate longer lasting protection that also elicits pathogen clearance. As $B p$ is a respiratory pathogen, it can be hypothesized that generating an immune response at the respiratory mucosa is necessary for protection against pertussis.

Infection with $B p$ localizes to respiratory epithelium primes the immune response against subsequent $B p$ infection by recruitment of antibody producing cells and tissue resident memory T cells (Trm) (16). Bacteria invading mucosal surfaces can induce inflammation resulting in subsequent production of $\operatorname{IgA}$ antibodies (17). Patients previously infected with $B p$ have developed IgA antibody titers in their nasal secretions (18). In addition, anti$B p \lg A$ antibodies from patients who have convalesced from $B p$ infection inhibit bacterial attachment in vitro and increase $B p$ uptake and killing by human polymorphonuclear leukocytes $(19,20)$. Convalescent humans also generate $B p$ specific IgG antibody titers that have shown to correlate with protection $(21,22) . B p$ infected mice generate $B p$ specific CD4+ $\mathrm{T}$ cells in the lung that secrete IFN-y and $\operatorname{IL}-17(23,24)$. In mice, $B p$ infection induced Trms in the lung and were associated with pathogen clearance (16). Nonhuman primates previously infected with $B p$ generate both Th1 and Th17 memory cells that are still detected two years post infection (25). To induce a similar protective immune response that is observed during natural infection, mucosal vaccination has recently been investigated $(26-28)$ 
Mucosal immunization has been scarcely used as a vaccination strategy to generate a protective immune response against pertussis in clinical and pre-clinical models. Oral vaccination with wP induced the production of $B p$ specific antibody titers in both the saliva and serum of newborns (29). In a subsequent study in 1985, oral immunization of $10^{12}$ CFUs of killed $B p$ led to the induction of antibody titers in the saliva and serum of newborns (30). The frequency of pertussis was lower in orally vaccinated newborns during the first year of life compared to newborns who were unvaccinated although, this difference disappeared by the end of the year (30). In mice, oral administration of attenuated bacterial vectors Salmonella typhimurium and Escherichia coli expressing Bp antigen, filamentous hemagglutinin (FHA), results in the production of anti-FHA IgA antibody titers in the lung (31). Intranasal immunization of a live attenuated vaccine strain of $B p$, BPZE1 was protective both in preclinical and clinical studies (32-35). Previously, our lab has shown that IN immunization of DTaP can induce a protective immune response in mice $(27,28)$. Boehm et al $(2019)$ illustrated that IN vaccination of DTaP with and without the addition of the adjuvant curdlan induces both anti-Bp and anti-pertussis toxin (PT) IgG antibody titers, as well as Bp specific IgA in the lung (27). A subsequent study performed by Wolf et al (2021) suggested that IN-aP vaccination is protective through the induction of humoral responses 6 months after booster vaccination and challenge (28). Mounting evidence supports that mucosal immunization can be protective against $B p$ colonization, but murine studies lack the ability to evaluate one the of hallmark symptoms of pertussis, Bp-induced coughing. 
The rat model of pertussis has been utilized to characterize $B p$ pathogenesis and evaluate coughing manifestation from $B p$ infection (36-42). Only a few studies have been performed investigating vaccine efficacy in the coughing rat model of pertussis. Hall et al (1998) demonstrated that the SmithKline Beecham 3-component aP vaccine (detoxified PT (PTd), FHA, and a 69kDa antigen, presumably pertactin (PRN)), the Connaught 5component vaccine (PTd, FHA, agglutinins 2+3 (fimbriae), and PRN), and Evans wholecell pertussis vaccine protected against cough upon intrabronchial $B p$ challenge (41). Rats administered one single human dose of DTP had a lower incidence of coughing following $B p$ challenge (39). We hypothesized that both oral and IN vaccination would protect against bacterial burden upon challenge as well as protect against $B p$ induced coughing in the rat model of pertussis by generating a protective immune response at the site of infection. To test this hypothesis, we IN and OG vaccinated and challenged Sprague-Dawley rats with $1 / 5^{\text {th }}$ human dose of DTaP. IM-wP and IM-aP vaccinated and $B p$ challenged rats were used as positive controls to compare vaccine-mediated immunity as IM administration of DTaP is the current route of vaccine administration.

In our study, we aimed to use the rat model of pertussis to measure protection induced from mucosal vaccination of DTaP. By utilizing the coughing rat model of pertussis, protection against bacterial burden in the respiratory tract and prevention of $B p$ induced cough after immunization was critically analyzed. We also focused on evaluating the serological responses regarding vaccination followed by $B p$ challenge. Our data supports that protection can be afforded by mucosal immunization with DTaP. IN and OG immunization with DTaP not only induced systemic anti-Bp IgG antibodies but also 
induced mucosal anti-Bp IgA antibodies. These data suggest that oral vaccination of DTaP can generate a humoral immune response at the respiratory mucosa in rats. IN-aP and OG-aP was also capable of protecting against $B p$-induced cough. Furthermore, mucosal vaccination protected against bacterial burden in the respiratory tract. In conclusion, this study highlights the benefits of using the coughing rat model of pertussis to study mucosal vaccination with $B p$ vaccines.

\subsection{Results}

\section{Intranasal immunization induces systemic anti-Bp IgG and anti-PT IgM and IgG antibody titers following booster immunization.}

We hypothesized that IN-aP and OG-aP immunization would induce systemic IgM and IgG antibodies, as mucosal immunization stimulates the induction of both systemic and mucosal antibodies $(27,28,30)$. To test this hypothesis, 3-week-old Sprague Dawley rats were IM-wP, IM-aP, IN-aP, and OG-aP immunized followed by a booster vaccine at 6 weeks of age with the same corresponding vaccine (Fig. S13). Mucosal and systemic antibodies were measured over the course of vaccination (Fig. S13). Minimal differences in antibody titers prior to booster vaccination was observed for all immunized groups (Fig. 15). Compared to all other groups, IM-wP vaccination induced a significant increase of anti-Bp IgM in the serum 1-week post-booster vaccination (Fig. 15A). IM-wP, IM-aP, and IN-aP vaccinated rats had a significant increase in anti-Bp $\lg G$ antibody titers following booster immunization compared to mock vaccinated challenged (MVC) rats (Fig. 15B). Rats vaccinated via IM-aP had a 100 -fold significant increase in anti-PT IgM antibody titers one week post booster vaccine, compared to the MVC control (Fig. 15C). IM-aP and IN-aP vaccinated rats had a significant increase in anti-PT IgM antibodies compared 
to IM-wP, and OG-aP vaccinated rats (Fig. 15C). This same trend was also observed in measuring anti-PT IgG antibodies (Fig. 15D). Here our data show that following booster immunization, IN-aP vaccinated rats developed systemic anti-Bp $\lg G$ and anti-PT IgG and IgM antibody titers.

\section{Mucosal vaccination protects against cough from $B p$ infected rats.}

In 2014, Warfel et al showed that aP vaccination was protective against pertussis disease, but failed to protect against colonization and transmission of $B p$ in the nonhuman primate model (14). We hypothesized that mucosal vaccination with DTaP would protect against $B p$ induced cough by eliciting a protective immune response at the respiratory mucosa. To test this hypothesis, vaccinated rats were subsequently intranasally challenged with Bp 2-weeks post-booster vaccine administration (Fig. S13). Every evening postchallenge, coughs were counted using whole-body plethysmography (WBP). MVC rats averaged a total of five coughs or less during monitoring for the first 5 days of infection (Fig. 16A). At days 6 and 7 post-challenge, average coughs per fifteen minutes increased to more than thirty coughs (Fig. 16A). There was a significant decrease in coughs for rats vaccinated with IM-wP, IM-aP, IN-aP, and OG-aP at days 6 and 7 post-challenge compared to MVC rats (Fig. 16B-E). On average, rats vaccinated with IM-wP coughed approximately 6 coughs per fifteen minutes each day (Fig. 16B). Rats vaccinated with IM-aP on average coughed 2 times per fifteen minutes each day (Fig. 16C). IN-aP immunized rats on average coughed 3 times per fifteen minutes each day, while OG-aP vaccinated rats coughed on average 4 times per fifteen minutes each day (Fig. 16D\&E). To compare the average total number of coughs each day post-challenge, we calculated 
the total number of coughs for each group per animal. We observed a significant decrease in total number of coughs in rats vaccinated with IM-aP (14 coughs) and IN-aP (27 coughs) compared to MVC rats (102 coughs) over the nine-day infection (Fig. 16F). OGaP immunized rats coughed on average 35 times (Fig. 16F). Our data demonstrates that mucosal vaccination in rats protects against $B p$ induced cough.

\section{Intranasal vaccination protects against pulmonary distress.}

Our previous work has shown that $B p$ infected rats had a significant increase in pulmonary distress following challenge (43). Pulmonary distress can be evaluated by calculating enhanced pause $(\mathrm{PenH})$. PenH functions as a representation of bronchoconstriction taking into consideration the timing between early and late expiration and the estimated maximum inspiratory and expiratory flow per breath. We hypothesized that mucosal vaccination would protect against $B p$ induced pulmonary distress, as bacterial clearance would decrease inflammation. Here, rats vaccinated with IM-wP, IM-aP, and IN-aP had a significant decrease in PenH compared to the MVC control group at days 5 and 7 postchallenge (Fig. 17A-D). Rats vaccinated IM-aP and IN-aP also had a significant decrease in PenH at day 6 post-challenge compared to MVC rats (Fig. 17A\&C-D). However, there was no significant decrease in PenH in OG-aP vaccinated rats compared to MVC suggesting that the induced immune response is not sufficient enough to protect rats from $B p$ induced respiratory distress (Fig. 17E). Other respiratory parameters were also measured using WBP (Fig. S14). In brief, we observed that rats vaccinated IM-wP, IM$\mathrm{aP}$, and IN-aP had a significant decrease in pause (PAU) compared to MVC control group,

which is another indicator of bronchiole restriction (Fig. S14B). Rats vaccinated with the 
aP regardless of route also had a significant decrease in Tidal volume (TVb) compared to MVC rats, which could be crudely associated with inflammation (Fig. S14D). Overall, our data demonstrated that $\mathrm{IN}-\mathrm{aP}$ vaccination decreases pulmonary distress of $B p$ infected rats.

\section{Mucosal immunization induces production of $B p$ specific antibodies in the serum,} while intranasal immunization also induces PT specific antibodies in the serum.

Next, we wanted to measure systemic antibody responses to $B p$ and PT following challenge. IM-wP vaccinated rats had a slight increase in anti-Bp IgM antibodies compared to all other vaccinated groups, albeit not significant (Fig. 18A). We observed a significant increase in anti-Bp IgG antibody titers in IM-wP, IM-aP, and IN-aP vaccinated rats compared to the MVC at day 1 post challenge (Fig. 18B). At day 9 post-challenge, all vaccinated rats had a significant increase of anti-Bp $\lg G$ antibody titers compared to the MVC control (Fig. 18B). Following Bp challenge, IM-aP and IN-aP immunized rats had a significant increase in anti-PT IgM antibodies compared to IM-wP immunized rats and MVC control. (Fig. 18C). Similar results were observed in measuring anti-PT IgG titers (Fig. 18D). IM-aP and IN-aP vaccination induced a significant increase in anti-PT IgG antibody titers compared to MVC, IM-wP, and OG-aP immunized rats after booster vaccination and at days 1 and 9 post-challenge (Fig. 18D). Although not significant, two of the OG-aP immunized rats had detectable anti-PT IgG antibody titers in the serum at day 9 post-challenge (Fig. 18D). Enzyme-linked immune absorbent spot (ELISpot) assay was used to determine the number of $B p$ specific $\lg G$ cells in the bone marrow at day 9 post-challenge. There was an increase in the number of $B p$ specific IgG cells in the bone 
marrow in all vaccination groups compared to the MVC control; however, the only significant increase in $B p$ specific lgG producing cells in the bone marrow was detected in IM-wP vaccinated rats (Fig. S15). Our data indicate that mucosal vaccination via IN and $O G$ immunization induced $B p$ specific IgG antibody responses.

Intranasal immunization induces production of Bp specific IgA antibodies in the nasal cavity

In humans, previous $B p$ infection leads to anti-Bp IgA antibodies in nasal secretions (18). IgA antibodies to $B p$ play a role in the inhibition of $B p$ attachment in vitro to epithelial cells (19). Here, we investigated if IN and OG immunization of DTaP would induce mucosal IgA antibodies in the lung and/or the nasal cavity. In the lung, three of the four IN-aP vaccinated rats had detectable anti-Bp $\lg A$ antibodies at day 1 post-challenge, although not significant (Fig. 19A). We did not detect anti-Bp IgA antibody titers at day 1 postchallenge in the lung of IM-wP, IM-aP, or OG-aP vaccinated rats (Fig. 19A). Low levels of anti-Bp $\lg A$ titers were measured in all vaccinated groups at day 9 post-challenge albeit not significant compared to our MVC control (Fig. 19A). The same trend was observed in the lung measuring anti-PT IgA titers at day 1 post-challenge (Fig. 19B). At day 9 postchallenge $50 \%$ of $\mathrm{IN}-\mathrm{aP}$ rats and $25 \%$ of rats OG-aP vaccinated had detectable anti-PT IgA (Fig. 19B). In the nasal cavity, only one IN-aP vaccinated rat had detectable anti-Bp $\lg \mathrm{A}$ antibody titers; however, we did measure a significant increase in anti- $B p \lg A$ antibodies in IN-aP immunized rats at day 9 post-challenge compared to the MVC, IMwP, IM-aP, and OG-aP immunized rats (Fig. 19C). Only one IN-aP and one OG-aP vaccinated rat had detectable amounts of anti-PT IgA in the nasal cavity at day 9 post 
challenge (Fig. 19D). Overall, our data reveal that IN-aP immunization is capable of inducing IgA antibodies in the nasal cavity of rats.

\section{Mucosal immunization protects against acute inflammation in the lung.}

Our previous rat challenge study illustrated that intranasal $B p$ challenge gave rise to both acute and chronic inflammation in the rat lung (43). Here, we used histology to assess if mucosal immunization would protect against $B p$ induced inflammation in the lung. At day 1 post-challenge, no differences in acute inflammation were observed; however, at day 9 post-challenge, rats vaccinated with IM-aP, IN-aP, and OG-aP had a significant lower acute inflammation scoring compared to the MVC rats. (Fig. 20A\&C). IN-aP immunized rats had a higher chronic inflammatory score at day 1 post-challenge (Fig. 20B\&D). There were no observed differences in chronic inflammation in vaccinated rats compared to MVC rats at day 9 post-challenge (Fig. 20D). Total inflammation was calculated by combining both acute and chronic inflammatory scores, as rat lungs exhibited both types of inflammation. No differences in total inflammation score were observed at day 1 postchallenge; however, all vaccinated rats had a significant lower total inflammation score compared to MVC rats (Fig. 20E). There were no differences in lung weight, which can be used as a crude measurement for lung inflammation, following Bp challenge. (Fig. S16A). We did observe a significant increase in percent body weight change in IM-wP vaccinated rats compared to MVC control rats suggesting that WP protects against weight loss observed in non-vaccinated challenged rats (Fig. S16B). Our observations suggest that that mucosal vaccination protects against $B p$ induced inflammation in the lung (Fig. 20C\&E). 


\section{Mucosal vaccination protects against $B p$ challenge.}

Next, we wanted to assess if mucosal immunization could protect against $B p$ burden in the respiratory tract. Bacterial burden in the respiratory tract was determined $1 \mathrm{hr}, 1-$, and 9- days post $B p$ challenge. Bacterial burden was measured at $1 \mathrm{hr}$ post-challenge $(n=2)$ to assess potential bacterial loss for our original challenge dose. In the lung, trachea, and nasal lavage fluid, we measured approximately $10^{6}$ CFUs $1 \mathrm{hr}$ post challenge (Fig. 21AC). At day 1 post-challenge there was a significant $98.5 \%$ reduction in bacterial burden in the lung of IM-aP immunized rats compared to MVC (Fig. 21A). IM-wP. IM-aP, IN-aP, and OG-aP vaccinated rats all had a significant decrease in bacterial burden in the lung at day 9 post-challenge compared to MVC rats (Fig. 21A). There was also a significant decrease in bacterial burden in the trachea at both days 1 and 9 post-challenge in all vaccinated rats compared to MVC (Fig. 21B). At day 1 post-challenge, there was a significant $86-97 \%$ reduction in bacterial burden in all vaccinated rats compared to MVC rats in the nasal cavity (Fig. 21C). At day 9 post-challenge we did not measure any significant differences between groups as most of the bacteria were cleared from the nasal cavity (Fig. 21C). Overall, we observed that IN-aP and OG-aP vaccinated rats have a significant reduction in bacterial burden in the respiratory tract compared to the MVC control group at days 1 and 9 post-challenge (Fig. 21A-C).

wP immunization induces a proinflammatory cytokine response compared to mucosal vaccinated Sprague-Dawley rats. 
Previous studies have shown that both wP immunization and Bp infection induces a proinflammatory Th1/Th17 immune response, while aP immunization promotes a more Th2 skewed response (44-50). In our current study, we measured cytokines in the lung and serum induced from vaccination and challenge. In the lung at day 1 post-challenge, we measured a significant 4-fold increase in IL-17 in MVC rats compared to IM-aP and INaP vaccinated rats (Fig. 22A). At day 9 post-challenge, IM-wP immunized rats had a significant increase in IL-17 compared to IM-aP, IN-aP, and MVC rats (Fig. 22A). IM-wP vaccinated rats also had a significant increase in Th1 cytokine IL-12p70 compared to MVC rats in the lung and serum at day 9 post-challenge (Fig. 22A\&B). IM-wP vaccinated rats had a significant increase in Th2 cytokines IL-4 and IL-13 in the serum compared to IM-aP and IN-aP vaccinated rats and a significant increase in IL-4 to MVC control at day 9 post-challenge (Fig. 22B). IM-wP immunized rats also had a significant increase in GCSF in the serum day 9 post-challenge compared to IM-aP, IN-aP, OG-aP, and MVC rats. Overall, we did not observe marked changes in cytokine responses between DTaP vaccinated rats compared to the MVC control group; however, rats OG-aP immunized did have a slight increase in IL-17, though not significant. The observed difference in cytokines levels could be from $B p$ challenge rather than vaccination. The increase in acute inflammatory score in the lung of IM-wP immunized rats at day 9 post-challenge could be associated to the increase in proinflammatory cytokines. Graphs showing the statistical significance between cytokines in the serum and lung are in the supplementary data (Fig. S17\&18). Our data support that response to B. pertussis in IM-wP immunized animals is associated robust cytokine response compared to both naïve and aP 
vaccinated rats, which is to be expected based on the work that has examined the Th17 response induced by whole cell pertussis vaccines.

$B p$ infection induces an increase in circulating neutrophils in the blood, as well as white blood cells and lymphocytes (51-56). In our current study, we utilized hematology and flow cytometry to evaluate these populations. Hematology analysis revealed a significant increase in blood lymphocytes in the IN aP vaccinated rats compared to MVC postchallenge; however, no other differences in white blood cell counts in the blood were observed in the other vaccinated groups. (Fig. S19A\&B). At day 1 post-challenge, there was a significant decrease in circulating neutrophils in the blood for IN-aP immunized rats compared to MVC rats (Fig. S19C). Flow cytometry analysis observed minimal differences in the number of neutrophils and B cells at days 1 and 9 post-challenge in all groups (Fig. S19E\&F). Based upon these data, subtle differences in various circulating cell populations were observed following $\mathrm{IN}-\mathrm{aP}$ vaccination.

\section{Serological responses correlate with bacterial clearance in the respiratory tract.}

Currently no definitive correlates of protection (CoP) for vaccines to protect against $B p$ have been established (22). It is appreciated that Th17 responses as well as Trms correlate with strong protection in mice and baboons. Antibodies to PT/FHA/PRN do not always correlate with protection in humans. In an effort to more precisely define correlates, using the rat model, we aimed to utilize the coughing phenotype and bacterial burden to identify the nature of how each vaccine protects (OG/IN/IM; acellular or wP). Previous work in our lab performed by Wolf et al (2021) illustrated that serum anti-Bp, anti-FHA, and anti-PT IgG antibody titers in the serum following IN vaccination in mice 
correlate with the decrease in bacterial burden in the lung following $B p$ challenge (28). Previous studies have shown that serum anti-Bp $\lg G$ antibodies induced from wP vaccination correlate with protection against bacterial burden in the lung of $B p$ challenged mice (57). Here, we hypothesized that antigen specific serum IgG and mucosal IgA antibodies correlate with decreased bacterial burden and cough, as IN-aP and OG-aP vaccination induced systemic and mucosal antibody responses. To test this hypothesis, we generated correlograms to evaluate both negative and positive correlations elicited by each vaccination route (58). Correlograms are an analysis tool that can be used to determine if the relationship observed between variables (i.e. bacterial burden and antibody titers) is random or not (59). If the relationship between the two variables is random the $\mathrm{R}^{2}$ correlation value is or near zero (59). The relationship is considered correlative if the $R^{2}$ values approximately positive or negative one (59). Significant positive nonzero correlation values demonstrate a positive correlation between variables, while significant negative nonzero values represent a negative correlation (59). Negative correlations are observed when two variables are inversely related to one another; that is, when one variable increases, the other decreases. With these data, as bacterial burden would drop, then the correlate would increase (negative correlation; inverse). A positive correlation would mean that as bacterial burden increases so does the correlate that is being compared to.

IM-wP vaccinated rats had strong negative correlations (protective) between serum anti$B p \lg G$ antibodies to both bacterial burden in the lung $\left(R^{2}=-0.97\right)$ at day 1 post challenge and the nasal cavity $\left(R^{2}=-0.84\right)$ at day 9 post-challenge (Fig. 23A-B). At day 1 post- 
challenge, we observed negative correlations (protective) between systemic anti-Bp IgM and anti-PT IgG antibodies in IM-aP vaccinated rats to bacterial burden in the nasal cavity $\left(R^{2}=-0.64,-0.64\right.$ respectively). Additionally, negative correlations were observed between anti-Bp IgM antibodies and bacterial burden in the trachea $\left(R^{2}=-0.72\right)$ at day 9 post-challenge (Fig. 23C-D). IM-aP vaccinated rats also had negative correlation between total cough counts over the course of challenge with IgG and IgM antibodies to $B p$ and PT (Fig. 23D). We expected that IN immunization would induce negative correlations between both serum- and mucosal-specific antibodies compared to bacterial burden in the lung, trachea, and nasal cavity at day 1 post-challenge (Fig. 23E). Lung anti-Bp IgA antibodies also negatively correlated with total cough count $\left(R^{2}=-0.74\right)$ and bacterial burden $\left(R^{2}=-0.6\right)$ in the lung at day 9 post-challenge for IN-aP vaccinated rats (Fig. 23F). We observed strong negative correlations between serum IgG and mucosal IgA antibody to bacterial burden in the lung $\left(R^{2}=-0.93,-0.93\right.$ respectively $)$ in OG-aP vaccinated rats at day 1 post-challenge despite the overall lower serological responses (Fig. 23G). At day 9 post challenge, there was a negative correlation between serum anti$B p \lg G$ antibodies to bacterial burden in the lung $\left(R^{2}=-0.81\right)$, trachea $\left(R^{2}=-0.49\right)$, and nasal cavity $\left(R^{2}=-0.52\right)$ in OG-aP immunized rats (Fig. 23H). We also noticed that OGaP vaccinated rats had negative correlations between serum $\lg G$ and mucosal $\lg A$ antibodies in the lung to total cough count $\left(R^{2}=-0.94,-0.84\right)$ at day 9 post-challenge (Fig. 23H). Positive correlations (non-protective) were observed between bacterial burden and total inflammatory score in IM-wP, IM-aP, and OG-aP immunized rats (Fig. 23A, C, G). Bacterial burden also positively correlated with total cough counts at day 9 post challenge (Fig. 23B, D, F, H). By utilizing correlograms, strong negative correlations between serum 
serological responses and bacterial burden were observed in IM-wP and IM-aP immunized rats. Additionally, our data underscore the idea that both systemic and mucosal antibodies correlate with the observed $B p$ clearance in the respiratory tract and protection from $\mathrm{Bp}$ induced cough elicited from IN-aP and OG-aP vaccination highlighting the observed differences between vaccination routes.

\subsection{Discussion}

The immunity induced by aP vaccines is relatively short lived; thus, DTaP/Tdap vaccinated individuals are still capable of $B p$ transmission $(60,61)$. We have recently reinvestigated the rat model of pertussis to further understand $B p$ pathogenesis from current circulating $B p$ strains (such as CDC isolate D420) (43). The coughing rat model of pertussis is a tool that can be used to evaluate bacterial burden in the respiratory tract, and also evaluate vaccine-induced immunity against cough and respiratory function (36$41,62)$. In our current study, we evaluated mucosal vaccination with DTaP in the coughing rat model of pertussis. To our knowledge, this study is the first to evaluate both IN and OG administered DTaP in the coughing rat model of pertussis. The data presented here suggest that, not only does mucosal immunization protect against bacterial burden, but also against $B p$ induced cough by WBP (Fig. 16\&21).

Vaccine mediated immunity has been studied in the coughing rat model of pertussis. Utilizing audio tape recorders, Hall et al (1998) illustrated that 3 and 5 component aP vaccines administered subcutaneously could protect against $B p$ induced cough (41). wP immunization administered intraperitoneally also decreased the incidence of cough in rats (39). Neither study noted protection against bacterial colonization. Here, in our study, 
WBP was used to investigate $B p$ induced cough in IN-aP and OG-aP vaccinated rats, as well as measure bacterial burden in the respiratory tract. Our data supports that mucosal administration of DTaP protects against bacterial burden in the respiratory tract and reduced $B p$-induced cough (Fig. 16\&21). In addition, IN-aP vaccination reduced bronchial constriction in the lung that is elicited by Bp infection (Fig. 18). Previous studies evaluating wP and aP vaccines in the rat model of pertussis used one human dose per rat for immunization prior to challenge $(39,41)$. In an effort to best model an appropriate human to rat dose, we utilized a $1 / 5^{\text {th }}$ human dose to prime and boost based on the relative sizes of rats compared to mice. We have reported $1 / 40^{\text {th }}$ human dose as protective in mice and rats are roughly $10 x$ the weight of mice (63). One caveat of this study is that we did not evaluate other human-to-rat titrations of aP. Identification of a minimal protective rat dose would allow for the investigation of vaccine efficacy of new potential antigens/adjuvants in this model (63).

Mucosal immunization has been of particular interest in the pertussis field. We and others have recently evaluated intranasal immunization of DTaP in Bp challenged mice $(27,28$, 64-66). Intranasally DTaP vaccinated mice were protected against $B p$ challenge and also generated both systemic and mucosal antibodies $(27,28)$. Live attenuated strain BPZE1 administered intranasally was protective against $B p$ challenge in mice and baboons, and is currently in Phase 2 of clinical studies $(33,34,67,68)$. BPZE1 immunization induces both anti-Bp IgG and IgA antibodies systemically and has an increase in resident memory T cells in the lung $(33,69)$. Oral immunization has also been investigated as a possible vaccination strategy against pertussis. Oral immunization of heat-inactivated $B p$ 
protected newborns against $B p$ challenge, as well as generated serum and saliva antibody titers (70). Recombinant technologies has led to the development of live attenuated Salmonella strains presenting $B p$ antigens $(71,72)$. Oral immunization of Salmonella typhimurium aro vaccine strain harboring the gene for PRN resulted in reduced bacterial colonization in the lung post $B p$ challenge (71). Salmonella dublin aro $A$ mutant expressing the gene for FHA was also orally administered as a vaccine in mice (72). Vaccination with this strain induced IgG and IgA antibody titers to FHA in the serum and gut (72). Our current study shows that mucosal immunization not only induced systemic anti-Bp lgG but also anti-Bp lgA antibodies that likely play role in clearance at the mucosa (Fig. 18-19, 21).

CoP is defined as the immune response that is statistically accountable for the observed protection (73). While no CoP has yet to be fully agreed upon against pertussis in humans, anti-PT IgG levels $>5 \mathrm{IU} / \mathrm{ml}$ are associated with protection in humans (74). In mice, IN administration of DTaP induced anti-Bp, anti-FHA, and anti-PT IgG antibodies while wP vaccination induced serum anti-Bp IgG antibodies that correlated with protection against $B p(28,57)$. Here in our study, we generated correlograms between all vaccinated groups to identify correlations between variables in the coughing rat model of pertussis, which has yet to be established (Fig. 23). Our results indicate that bacterial clearance in the lung, trachea, and nasal cavity negatively correlate with systemic anti-Bp and -PT IgM and anti-Bp IgG antibodies in IM-wP and IM-aP immunized rats, while systemic and mucosal antibodies correlated with bacterial clearance in IN-aP and OG-aP vaccinated rats (Fig. 23). Antibodies generated following immunization also negatively correlated 
with a decrease in total cough counts in vaccinated rats (Fig. 23). These results suggest that the increase in systemic and mucosal antibodies induced from IN and OG vaccination correlates with protection against $B p$ burden in the respiratory tract and $B p$ induced cough. It is important to note, that OG-aP immunized rats did not generate significant serum antibody titers to the whole bacterium until day 9 post-challenge (Fig. 18B). Also, two OG-aP immunized rats had an increase in anti-PT IgG antibody titers in the serum and anti-Bp IgA antibodies in the lung (Fig. 18D, 19A). We did however detect antigen specific $B$ cells in the bone marrow and 3 of the 4 rats had low levels of $\lg A$ in the lung in OG-aP immunized rats (Fig. S15). One caveat that should be mentioned is that we did not investigate the $T$ cell responses (Th1/Th2/Th17/Trm/Tem) in rats but future studies will incorporate this into the study design. We have proposed a summary for mechanism for oral vaccination of aP (Fig. S20). We hypothesize that this could be because limited amount of vaccine that successfully travels to the gut-associated lymphoid tissue (GALT) for the generation of an immune response. Oral vaccines have to travel through increased $\mathrm{pH}$ in the stomach while limited absorption and availability for antigen recognition also occur in the gastrointestinal tract (75). Increase in dose, number of doses, or delivering vaccine in an encased vehicle are potential methods to increase orally vaccinated immune responses. Targeting of vaccine to intestinal $\mathrm{M}$ cells for antigen presentation has also been shown to increase oral vaccine efficacy (76). Though we did not study new adjuvants here, we hypothesize adjuvants can aid in stimulating a protective mucosal immune response. These approaches could all potentially increase the efficacy observed through oral vaccination of aP. Furthermore, to deliver the vaccine to the gut, one could 
envision novel deliver mechanisms such as gelatin coated chewables similar to gummy vitamins that are now popular.

In summary, mucosal vaccination not only protected against bacterial burden in the respiratory tract of challenged rats, but also protected against $B p$ induced cough and respiratory distress measured by WBP (Fig. 16-17, 21). It is critical that "next generation pertussis" vaccines protect against bacterial colonization in the lung, nasal cavity, and trachea, as disease manifestations are dependent on bacterial colonization of the lung and trachea, mediated by FHA and fimbriae $(77,78)$. Both IN and OG immunized rats generated anti-Bp specific IgG antibodies in the serum, while IN vaccinated also generated significant anti-Bp IgA antibody titers in the nasal cavity following challenge (Fig. 18-19). IN-aP and OG-aP immunized rats were protected against acute and total inflammation in the lung (Fig. 20). Our data support the potential of a mucosal vaccination against $B p$.

Further work is needed to fully characterize the immune response generated following $\mathrm{IN}-\mathrm{aP}$ and $\mathrm{OG}-\mathrm{aP}$ vaccination in rats, as well as vaccine mediated immunity from vaccination in the coughing rat model of pertussis. T cell immune responses that have been shown to play a role in natural and vaccine mediated immunity against pertussis have yet to be evaluated in the coughing rat model of pertussis due to limited availability of resources to adequately measure $T$ cell responses. Vaccine mediated memory has yet to be evaluated in the coughing rat model of pertussis. Additional research is needed to critically assess vaccine mediated memory, as it is essential that next generation of 
pertussis vaccines induce longer lasting memory then current vaccines. Future work is also needed to evaluate mucosal immunization against current circulating strains of $B p$ as current strains are genetically divergent from strains of the past, with the goal of making the most efficacious vaccine against $B p$.

\subsection{Materials and methods}

Vaccine composition and administration. INFANRIX (GSK Cat. 58160-810-11) acellular pertussis human vaccine (DTaP) and the National Institute for Biological Standards and Control WHO whole cell pertussis vaccine (NIBSC code 94/532) was used for this study. Vaccines were diluted with endotoxin-free Dulbecco's PBS (Thermo Fisher Scientific Cat. TMS012A) to a concentration of $1 / 5^{\text {th }}$ human dose. Vaccines were diluted and administered no more than $1 \mathrm{hr}$. from composition. The first dose of vaccine was administered to three-week-old $(50 \mathrm{~g})$ female Sprague-Dawley rats (Charles River Cat. 001CD). At six weeks of age, a booster vaccine of the same dose was administered, followed by $B p$ challenge at eight weeks of age. Intramuscular (IM) vaccinated rats received $100 \mu$ in the right thigh muscle of the hind limb. Intranasal (IN) immunized rats were first anesthetized with isoflurane until breathing was minimal. Rats then received $50 \mu$ l of vaccine in each nostril for a $100 \mu$ dose. Oral gavage (OG) vaccinated rats received $100 \mu$ lose delivered curved 18 gauge feeding needle (Fisher Scientific Cat. NC9349775). MVC control group received 100 $\mu$ l of the same endotoxin free PBS used to dilute the vaccines in the right thigh muscles of the hind limb. One-week post-prime, twoweek post-prime, and one-week post-boost blood was collected via saphenous blood draws for serological analysis. 5mm animal lancets (Fisher Scientific Cat. NC9891620) was used for blood draw. Blood was collected in capillary tubes (Fisher Scientific Cat. 
NC9059691) for centrifugation. Blood was spun at 15,000x $g$ for 3 min., serum collected and stored at $-80^{\circ} \mathrm{C}$ until analysis.

Bordetella pertussis strains and growth conditions. Bp strain D420 was cultured on Bordet Gengou (BG) agar (Remel ${ }^{\mathrm{TM}}$ Cat. R45232) supplemented with $15 \%$ defibrinated sheep blood (Hemostat Laboratories Cat. DSB500) (1). Bacteria cultured BG plates incubated for $48 \mathrm{hrs}$ at $36^{\circ} \mathrm{C}$. Using polyester swabs (Puritan Cat. 22-029-574), Bp was transferred into $20 \mathrm{ml}$ Stainer-Scholte liquid media (SSM) in new $125 \mathrm{ml}$ flasks (Thermo Fisher Scientific Cat. FB500125) (79). Bacterial cultures were allowed to grow at $36^{\circ} \mathrm{C}$ for $24 \mathrm{hrs}$ inside a shaking incubator at $180 \mathrm{rpm}$.

Intranasal challenge. Vaccinated eight-week-old $\sim 200 \mathrm{~g}$ female Sprague-Dawley rats were then challenged. Bp was grown as illustrated above. Rats were anesthetized with ketamine and xylazine $50-100 / 5-10 \mathrm{mg} / \mathrm{kg}$ and challenged with $10^{8} \mathrm{CFUs}$ in $100 \mu \mathrm{l}$ intranasally, $50 \mu \mathrm{l}$ in each nostril. Body weight of each rat was recorded before bacterial challenge, and body weights were taken post-euthanasia to calculate percent weight change. At days 1 and 9 post challenge, rats were then euthanized. Upon euthanasia blood was collected via cardiac puncture and transferred into ethylenediaminetetraacetic acid (EDTA) (BD Cat. 365974) and serum separation (BD Cat. 026897) tubes. Following cardiac puncture, $250 \mu \mathrm{l}$ of blood was collected into EDTA tubes for flow cytometry and ProCyte (IDEXX) analysis, while remaining blood was collected in serum separation tubes to isolate the serum via centrifugation $(15,000 \times \mathrm{g}$ for $3 \mathrm{~min})$ and used for serological and cytokine analysis. To determine bacterial burden in the respiratory tract, the lung and 
trachea was excised separately and homogenize. Lung weights were recorded following excision before homogenization. Lungs were then collected in gentleMACS C tubes (Miltenyi Biotec Cat. 130-096-334) in 2ml of PBS and homogenized using Miltenyi Biotec tissue dissociator (Cat. 130-095-927). Polytron homogenizer was used to homogenize the trachea in $1 \mathrm{ml}$ PBS. Bacterial burden in the nares was determined by flushing $2 \mathrm{mls}$ of sterile 1x PBS through the nares and collected for serial dilution and plating. Serial dilutions of the homogenates and nasal collection were plated on BG plates supplemented with ceftibuten (Sigma-Aldrich Cat. SML0037) $10 \mu \mathrm{g} / \mathrm{ml}$.

Serological analysis. Enzyme-linked immunosorbent assays (ELISA) was used to measure antibody titers of vaccinated and infected rats. Bp specific whole bacteria ELISA plates were coated with $50 \mu \mathrm{l}$ of $10^{8} \mathrm{Bp}$ grown as mentioned above for infection. Antigen specific antibody titers to PT (List Biological Laboratories \#180) were measured by coating ELISA plates with $50 \mu \mathrm{l}$ of antigen per well. Antigen coated plates incubated over night at $4^{\circ} \mathrm{C}$. After incubation, plates were washed with 1x PBS-Tween 20 and blocked with $5 \%$ skimmed milk for 2 hrs at $37^{\circ} \mathrm{C}$. Following blocking, ELISA plates were washed and serum from the saphenous blood draws and blood collected from cardiac puncture post-euthanasia were serially diluted down the ELISA plate and incubated for 2 hrs at $37^{\circ} \mathrm{C}$. To measure respiratory $\lg \mathrm{A}$ antibody titers in the lung and nasal lavages, lung homogenate supernatant and nasal lavage was added and incubated for $2 \mathrm{hrs}$ at $37^{\circ} \mathrm{C}$. After incubation, ELISA plates were washed as described above and $100 \mu \mathrm{l}$ of secondary goat anti-rat IgG (SouthernBiotech Cat. 3030-04), goat anti-rat IgM (SouhternBiotech Cat. 3020-04), or goat anti-rat IgA (MyBioSource Cat. MBS539212) was added to the plates at a dilution of $1: 2,000$ in PBS $+5 \%$ milk and incubated for $1 \mathrm{hr}$ at $37^{\circ} \mathrm{C}$. Plates were then 
washed again and $100 \mu \mathrm{l} p$-nitrophenyl phosphate substate (Thermo Scientific Cat. 37620) was added and the plate was developed for $30 \mathrm{~min}$ at room temperature. After development, colorimetric signal of the ELISA plate at $A_{450}$ was measured by a Biotek Synergy H1 microplate reader. Antibody titers were considered positive if values were higher than the baseline. Baseline value for each sample was set as double the average value of the blank, in which no serum, lung supernatant, or nasal lavage added to these well. Limit of detection was set at 50 , and any samples with a titer value less than that were set to 50 .

ELISpot assay. ELISpot assay (ImmunoSpot Cat. mTgG-SCE-1M/2) was used to analyze antigen specific B cells in the bone marrow. The right hind femur of the rat was removed and placed into Dulbecco's modified Eagle's medium (DMEM) and frozen at $80^{\circ} \mathrm{C}$ until analysis. Bones were then thawed in water bath at $37^{\circ} \mathrm{C}$, and immediately transferred into spin tubes and spun at 1,000x $g$ for 3 min to collect the bone marrow. Bone marrow was passed through a $70 \mu \mathrm{m}$ filter to create a single cell suspension. Cells were centrifuged at $350 \times g$ for 5 min and the cell pellet was resuspended in CTL test B Media (ImmunoSpot). D420 was cultured as described above and coated the 96-well ELISpot plate as described by ELISA. The plate incubated overnight at $4^{\circ} \mathrm{C}$. Plate was then washed with 1x PBS before cells were added. Three serial dilutions of cells $(1.25 \mathrm{x}$ $10^{6}, 3.13 \times 10^{5}$, and $1.56 \times 10^{5}$ ) cells added per well and incubated at $37^{\circ} \mathrm{C}$ overnight. Rabbit anti-Rat IgG antibody (Abcam Cat. ab6733) was used to replace the anti-murine IgG detection antibody that was with the kit. The rest of the protocol was followed as per the manufacturer's instructions. ELISpot plates imaged and analyzed using ImmunoSpot S6 Entry analyzer and CTL software. 
Analysis of cough and bronchiole restriction using whole-body plethysmography. Buxco® FinePointe ${ }^{\mathrm{TM}}$ Whole Body Plethysmography (WBP) (DSI) was used to quantify respiratory function during infection. Every day following $B p$ challenge and one day before challenge (5:00PM), rat respiratory profiles and coughs were measured. A 5 min acclimation period was used before measuring cough and other respiratory parameters. After acclimation the respiratory profile was recorded for 15 mins for each rat. Coughs were counted and represented over 15 mins. Enhanced pause $(\mathrm{PenH})$ was calculated which represents bronchiole restriction during breathing. Coughs were counted based on box flow changes of the subject with classical cough-like waveforms. Patented fuzzy logic criteria was used to detect and count coughs (80). Each cough in a multi-cough event was counted individually. Frequency (F), Tidal Volume (TVb), Pause (PAU), Minute Volume (MVb), Inspiratory time (Ti), and expiratory time $(\mathrm{Te})$ were also collected and analyzed during the course of infection.

Histological assessment of the lung. The left lobe of the lung was used for histological assessment. Following excision of the left lobe, the sectioned portion was fixed in $10 \%$ formalin $48 \mathrm{hrs}$ at $26^{\circ} \mathrm{C}$. Following fixation, samples were embedded in paraffin and stained with H\&E by the WVU Pathology Department. Stained samples were used to characterize and score inflammation of the lung. All scorings were done by a boardcertified pathologist (iHisto). Individual scores were based on a standard qualitative scoring criterion: (0 - none, 1 - minimal (rare), 2 - mild (slight), 3 - moderate, 4 - marked, 5 -severe). The presence of neutrophils in the parenchyma, blood vessels, and airway was used to score acute inflammation, and chronic inflammation was characterized by 
mononuclear infiltrates of the parenchyma, blood vessels, and airway. All examination and scoring were done with no knowledge of the groups.

ProCyte analysis of blood. Blood from the EDTA tubes was used to analyze white blood cell, neutrophil, and lymphocyte counts. $25-50 \mu \mathrm{l}$ of blood was drawn from the EDTA tubes and analyzed by the Procyte. After ProCyte analysis the rest of the blood was used for flow cytometry analysis.

Flow cytometry analysis. Blood samples were lysed with 1x Pharmylse buffer (BD Biosciences Cat. 555899) for 20 min at room temperature. Blood samples were vortexed periodically during the 20 min incubation. After lysis, cells were resuspended in RPMI + $10 \%$ FBS to neutralize the lysis buffer and centrifuged at $1,000 \mathrm{~g} \times 5$. Cells were then washed with the RPMI $+10 \%$ FBS again. Cells were then resuspended in $1 \% F B S+P B S+5 m M$ EDTA. Blood samples were then blocked with anti-CD32 (BD Pharmingen Cat. 550270) antibody for $15 \mathrm{~min}$ at $4^{\circ} \mathrm{C}$. After blocking, the cells were labeled; CD45 Alexa flour 700 (Biolegend Cat. 202218), CD161 APC (Biolegend Cat. 205606), CD45R PE Cy7 (eBioscience Cat. 25-0460-82), His48 FITC (eBioscience Cat. 11-0570-82), CD43 PE (Biolegend Cat. 202812), and CD3 VioGreen (Miltenyi Biotec Cat. 130-119-125) (81). Samples were incubated $1 \mathrm{hr}$ at $4^{\circ} \mathrm{C}$ in the dark. To prepare the lung samples for flow cytometry, the lung homogenate was filtered through a $70 \mu \mathrm{m}$ cell strainer (BioDesign Cell MicroSives Cat. N70R). The suspension was centrifuged at 1,000 $x g$ for 5 min. The pellet was resuspended in Pharmlyse buffer and incubated at $37^{\circ} \mathrm{C}$ for 2 min. After incubation, the cells were centrifuged at $1,000 \times g$ for $5 \mathrm{~min}$, lysis buffer removed, and cells were blocked and labeled with antibody as described for blood samples. Both blood and lung samples were centrifuged at $1,000 \times g$ for 5 min and the 
pellets were resuspended in $0.4 \%$ paraformaldehyde and stored overnight at $4^{\circ} \mathrm{C}$. Samples were washed with $1 \mathrm{x}$ PBS $+5 \mathrm{mM}$ EDTA+1\%FBS and resuspended in $1 \mathrm{x}$ PBS +5mM EDTA+1\%FBS for analysis. Cell samples were analyzed on a LSR Fortessa and samples were gated and analyzed using FlowJo v10.

Cytokine analysis. Lung homogenates were centrifuged at $19,000 \times g$ for 4 min and the resulting supernatant was removed and stored at $-80^{\circ} \mathrm{C}$ until further analysis. Lung supernatant and serum cytokines were measured using a ProcartaPlex Multiplex Immunoassay kit: Th Complete 14-Plex Rat ProcartaPlex Panel (Thermo Fisher Scientific Cat. EPX140-30120-901) per the manufacturer's instructions. Cytokines with bead counts less than 35 were invalidated.

Generation of correlograms. Correlograms were created using R Studio software. Pearson correlation coefficients were calculated between each set of variables listed in the master table and then illustrated in the representative plot for each vaccine route and timepoint.

Statistical analysis. GraphPad Prizm 7 was used to analyze the data. The minimum biological replicates for the challenge studies were three for MVC control group and four rats per vaccinated groups. For statistical comparisons between vaccinated groups and the MVC control group over the entire course of the infection, two-way analysis of variance (ANOVA) was used with Dunnett's post hoc test. One-way ANOVA was used for comparison between vaccinated groups and MVC for an individual day or timepoint with Dunnett's post hoc test. Kruskal-Wallis test with Dunnett's post hoc test compared between groups for mucosal IgA comparisons. ROUT test was used to identify any 
potential outliers during cytokine analysis of the lung.

Data availability. Data requests for figures provided can be addressed to the corresponding author.

Ethics statement. This challenge study was performed in accordance with our approved protocol by West Virginia University Institutional Animal Care and Use Committee (IACUC) protocol 1811019148.6.

\subsection{ACKNOWLEDGMENTS}

The work performed in this project was supported by the Vaccine Development Center at WVU-HSC through a Research Challenge Grant no. HEPC.dsr.18.6 from the Division of Science and Research, WV Higher Education Policy Commission. The project was also supported by NIH R01Al137155 (F.H.D). Part of the project was also supported by CDC Contract Broad Agency Announcement (BAA) 75D301-19-R-67835. Flow cytometry experiments were performed in the West Virginia University Flow Cytometry Core Facility, which is supported by the National Institutes of Health equipment grant number S10OD016165 and the Institutional Development Award (IDeA) from the National Institute of General Medical Sciences of the National Institutes of Health under grant numbers P30GM103488 (CoBRE) and P20GM103434 (INBRE).

JMH and GJB performed vaccination and bacterial challenge. JMH and GJB monitored rats by whole body plethysmography. All authors participated in the animal experiments. JMH and TYW prepared flow cytometry samples. JMH and MAW performed cytokine analysis. JMH performed ELISA assays. MAD constructed correlograms. JMH, $\mathrm{MB}$, and FHD contributed to experimental design. JMH wrote manuscript with critical revisions from all authors. 
The authors would also like to thank Jacqueline Karakiozis, Brice Hickey, and Mary Tomago-Chesney (Pathology/Histology Core Facility) for the preparation of lung slides and staining lung slides with H\&E for scoring analysis. The authors would also like to thank Dr. Kathleen Brundage (WVU Flow Cytometry \& Single Cell Core Facility) for assisting in flow cytometry and equipment instruction.

The authors would also like to acknowledge that figures S1 and S8 were created with BioRender.com 


\subsection{Figures}

A

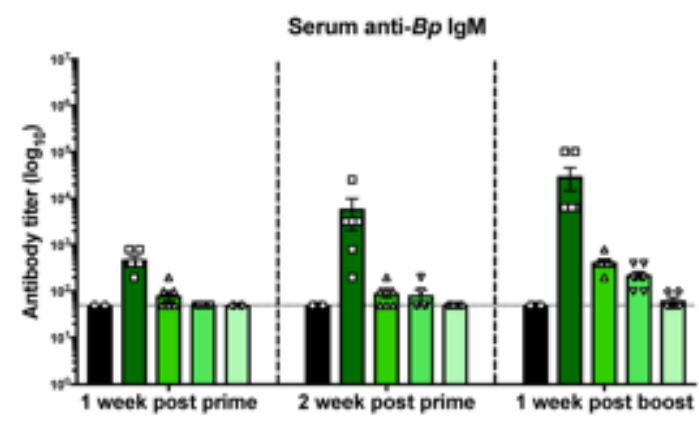

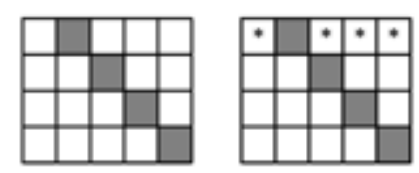

C

\begin{tabular}{|l|l|l|l|l|l|}
\hline IM-wP & & & & & \\
\hline IM-aP & & & & & \\
\hline IN-aP & & & & & \\
\hline OG-aP & & & & & \\
\hline
\end{tabular}

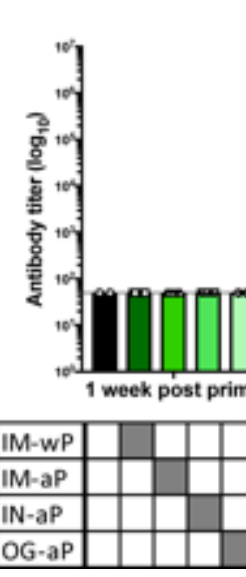

Serum anti-PT IgM

!
B
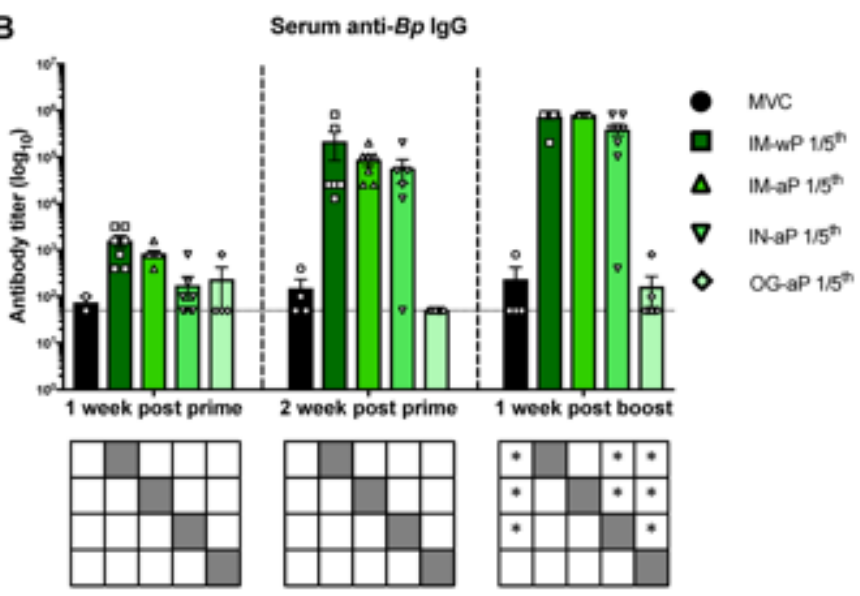

D

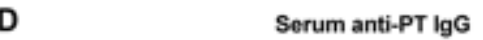

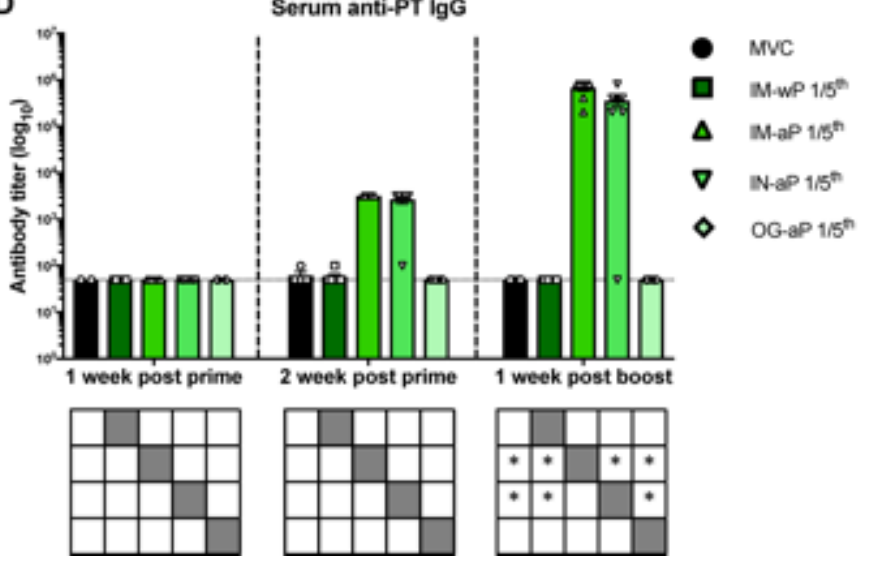

Figure 15. IN booster vaccination induces systemic anti-Bp and anti-PT antibody titers. 1 and 2 weeks post prime immunization and 1 week post boost blood was collected via saphenous vein, and anti Bp and anti PT IgM (A-C) and IgG (B-D) specific antibodies were measured. Results are shown on a log scale and as a mean \pm SEM $(n=3-8)$. Dotted line represents the limit of detection. ${ }^{*} P<0.05$. $(n=4-8)$. $P$ values were determined by two-way ANOVA with Dunnett's post hoc test compared between groups. * under each graph annotates the significance between labeled group under the y-axis and the group under the corresponding bar. Grayed out box annotates no stats calculated. 


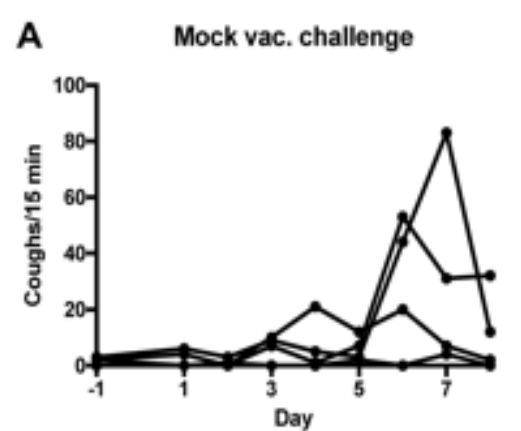

D

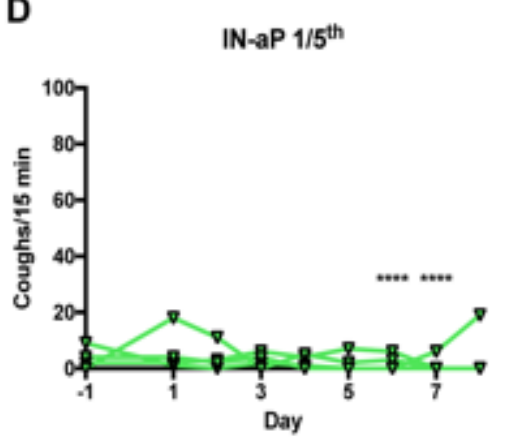

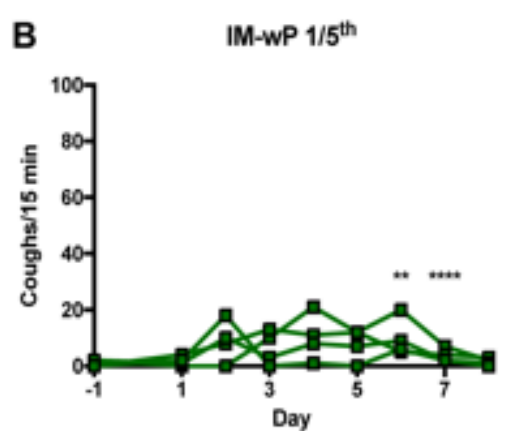

E

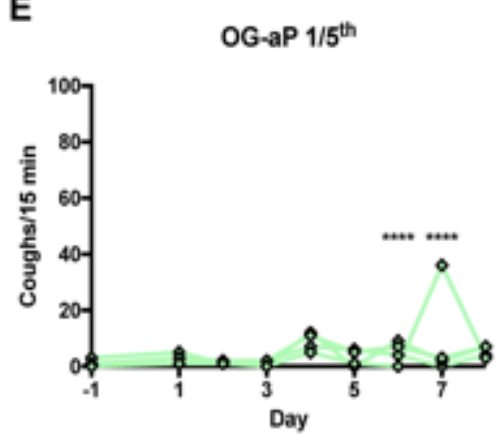

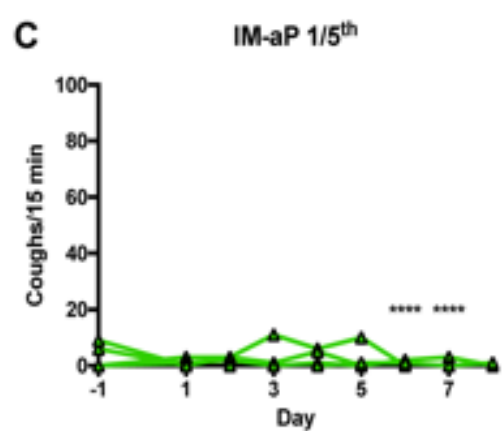

$\mathbf{F}$

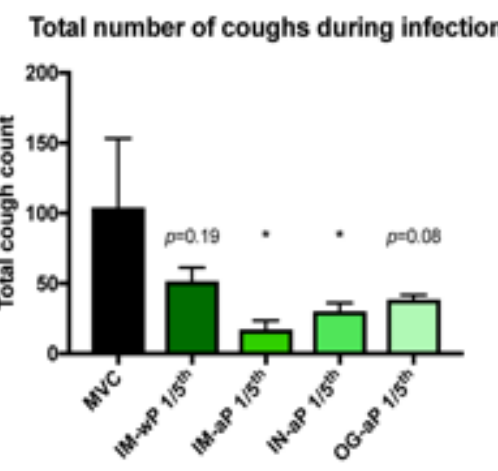

Figure 16. Intranasal and oral vaccination of acellular pertussis vaccine decreases cough of $B$. pertussis infected rats. Coughs were counted every day of the nine-day infection using whole body plethysmography. Coughs were counted for (A) mock vac. challenge rats, (B) IM-wP (C) IM-aP (D) IN-aP, and (E) OG-aP, vaccinated and challenged rats. To assess any potential differences between vaccine groups over the entire course of infection, $(F)$ average total number of coughs for each rat per group was compared. Results shown as mean \pm SEM $(n=3-4)$. $P$ values were determined by two-way ANOVA with Dunnett's post hoc test and one-way ANOVA with Dunnett post hoc test for total cough count, ${ }^{*} P<0.05,{ }^{* *} P<0.01,{ }^{* * *} P<0.001,{ }^{* * *} P<0.0001$ compared to the mock vac. challenged control group. 

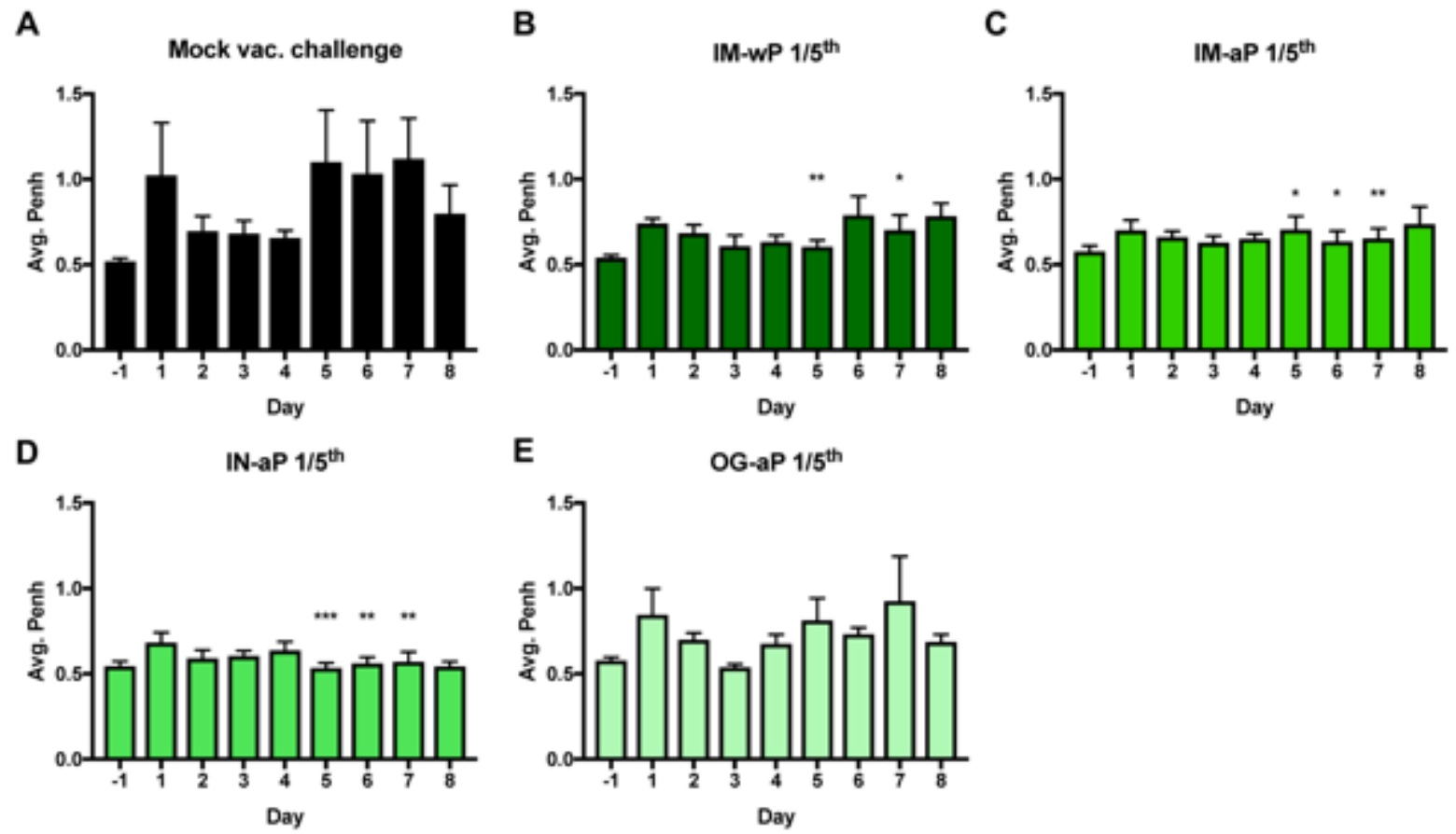

Figure 17. Intranasal vaccination decreases pulmonary restriction of Bordetella pertussis infected rats. Bronchiole restriction was measured over the course of infection by whole body plethysmography. Bronchiole restriction was determined by the factor Penh for (A) mock vac. challenge rats, (B) IM-wP (C) IM-aP (D) IN-aP, and (E) OG-aP vaccinated and challenged rats. Results shown as mean \pm SEM $(n=3-4)$. $P$ values were determined by two-way ANOVA with Dunnett's post hoc test, ${ }^{*} P<0.05,{ }^{* *} P<0.01,{ }^{* *} P<0.001$ compared to the mock vac. challenge group. 
A

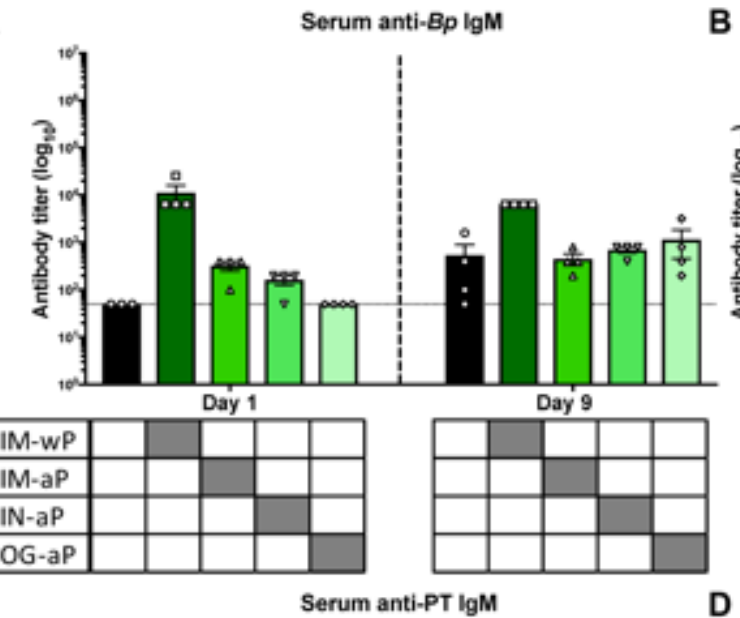

C

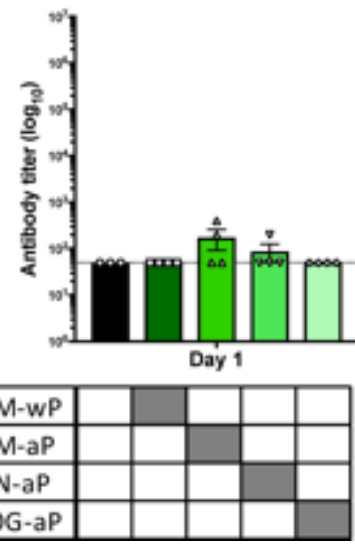

$B$

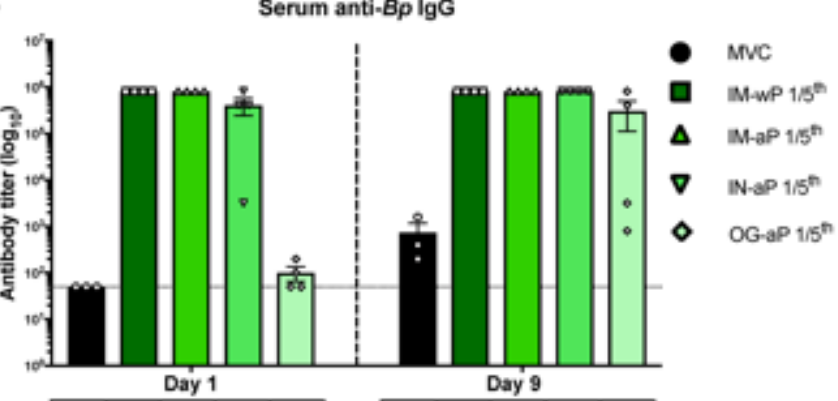

D

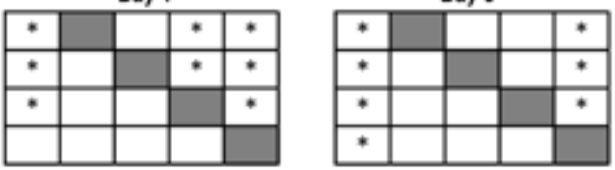

Serum anti-PT IgG

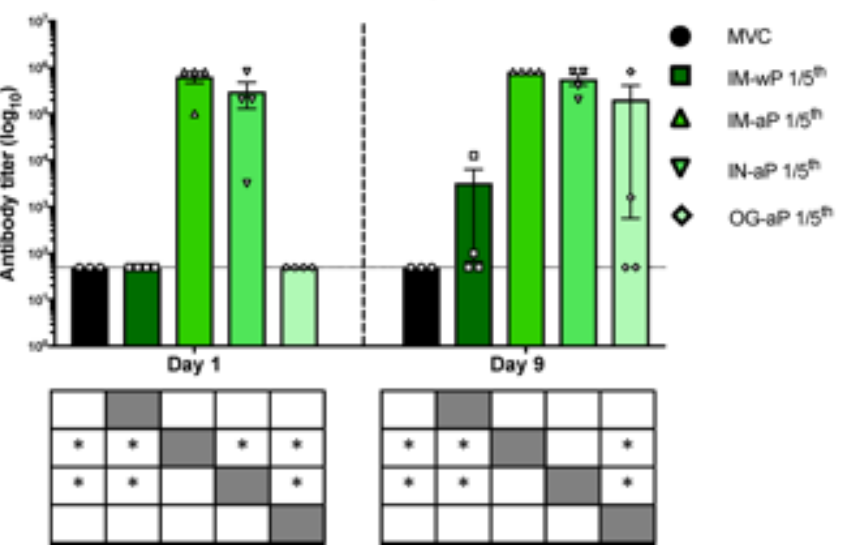

Figure 18. Mucosal vaccination induces production of anti- $B p \lg G$, while IN immunization also induces both anti-PT IgM and IgG antibodies. ELISAs were used to determine and compare the induced serological responses from vaccinated and challenge rats in the serum. Both $(A, C) \lg M$ and $(B, D) \lg G$ serum antibody titers from immunized and challenged rats were measured post prime, boost, and challenge. Dotted line represents the limit of detection. Results are shown on a log scale and as mean \pm SEM, ${ }^{*} P<0.05$ $(n=4)$. P values were determined by two-way ANOVA with Dunnett's post hoc test compared between groups. * under each graph annotates the significance between labeled group under the $y$-axis and the group under the corresponding bar. Grayed out box annotates no stats calculated. 
A

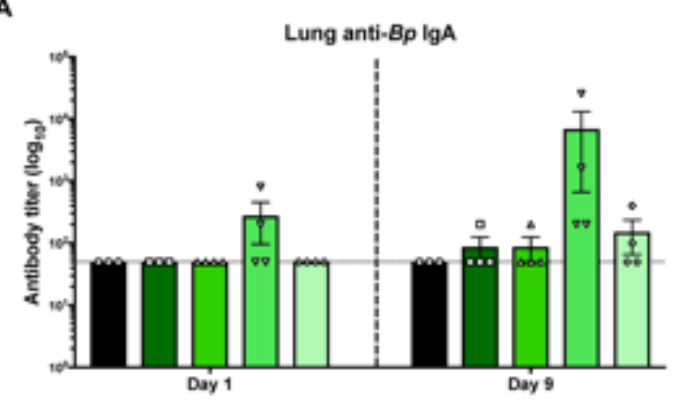

C

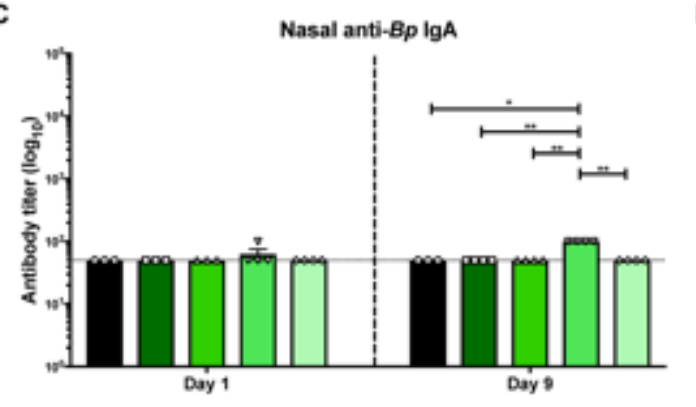

B

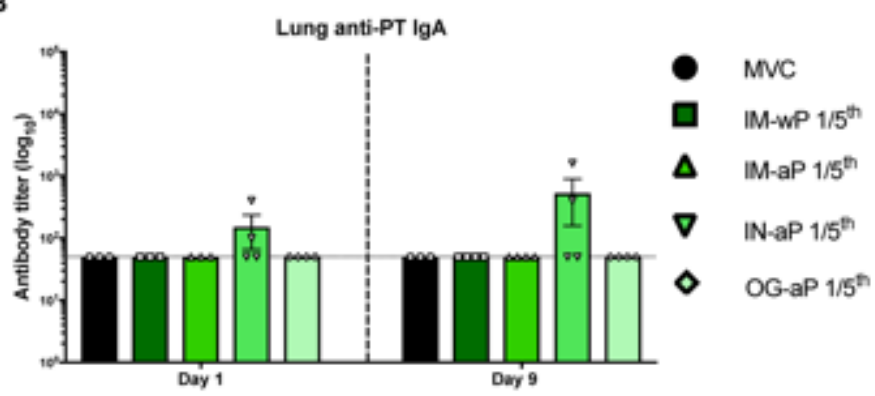

D

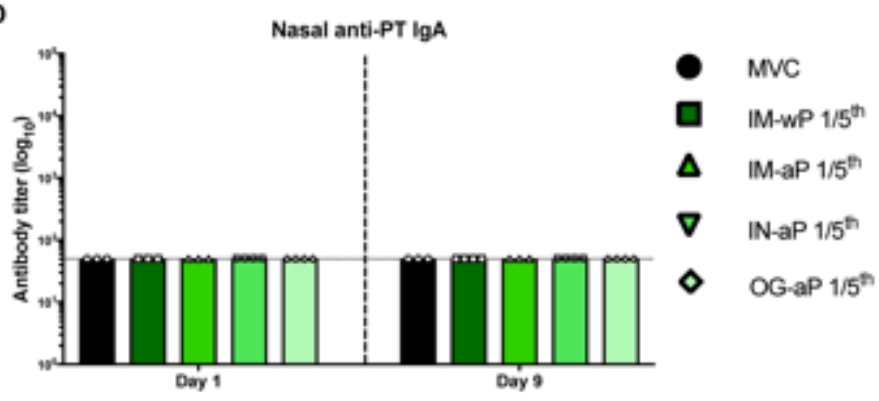

Figure 19. Intranasal immunization elicits the production of anti- $B p \lg A$ in the respiratory tract. ELISA was used analyze antibodies in the $(A, B)$ lung and $(C, D)$ nasal cavity from lung homogenate supernatant and PBS flushed through the nasal cavity from vaccinated and challenge rats at days 1 and 9 post challenge. IgA titers were determined against pertussis toxin and $B$. pertussis. Dotted line represents the limit of detection. Results are shown on a log scale and as a mean \pm SEM $(n=3-4) .{ }^{* *} P<0.01$, ${ }^{* * *} P<0.0001 P$ values were determined by Kruskal-Wallis test with Dunnett's post hoc test compared between groups. 
A

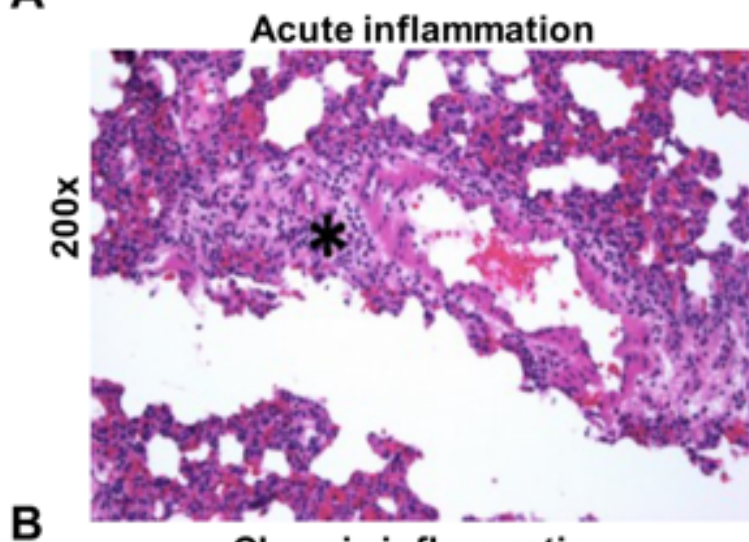

B

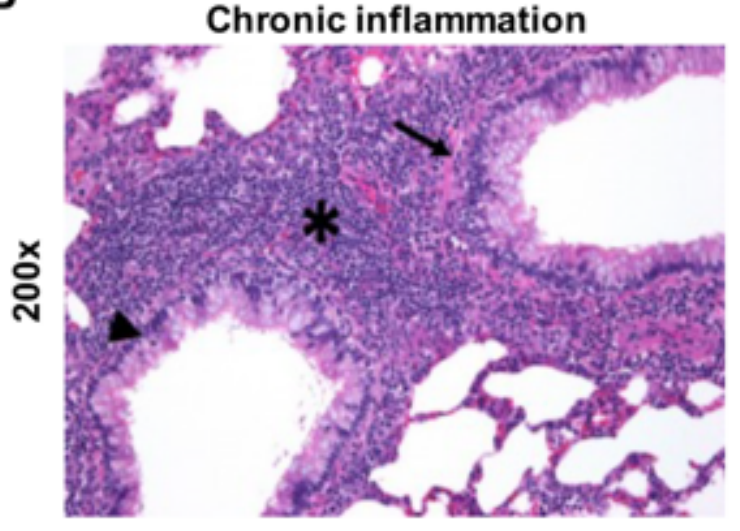

C

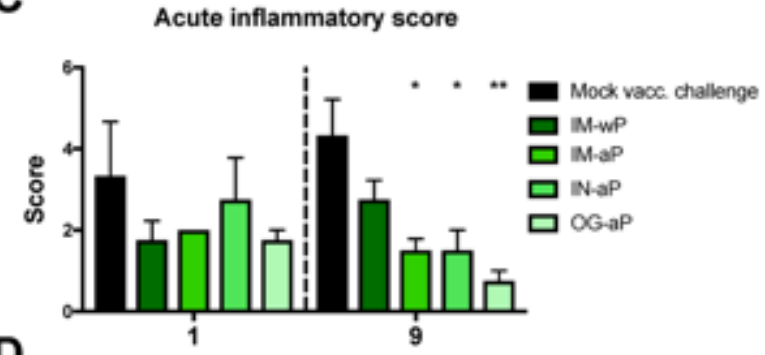

D

Chronic inflammatory score

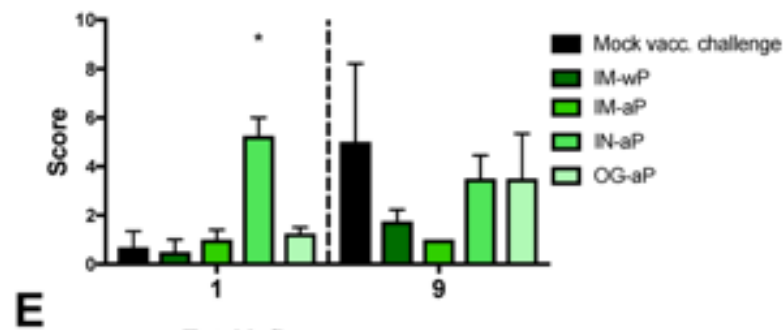

E

Total inflammatory score

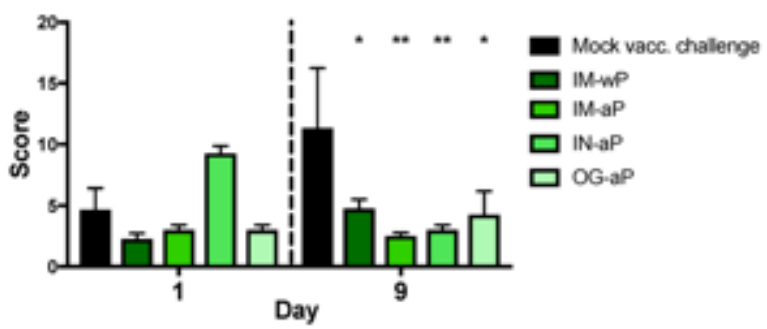

Figure 20. Mucosal vaccination protects against acute and total inflammation in the lung of $B p$ infected Sprague-Dawley rats. Post euthanasia, left lobe of the lung was excised, sectioned, and stained with hematoxylin and eosin. Lung samples scores were based on standard qualitative toxicologic scoring criteria (0 - none, 1 - minimal (rare), 2 - mild (slight), 3 - moderate, 4 - marked, 5 -severe). (A) Representative image of acute inflammation of rat lung showing increased numbers of neutrophils and edema surrounding blood vessel (asterisk). (B) Representative image of chronic inflammation of the rat lung showing increased numbers of mononuclear cells surrounding bronchioles (asterisk). Inflammatory cells are also present in the lamina propria (arrow) and epithelium (arrowhead) of bronchioles. (C) Average acute inflammation scores of the lung are detailed by the presence of neutrophils in the parenchyma, blood vessels, and the airways. (D) Average chronic inflammation scores are distinguished by mononuclear infiltrates in the parenchyma, blood vessels, and airway of the lung. $(E)$ Total inflammatory score calculated by the sum of the acute and chronic inflammation score of the lung. All 
scoring assessments were determined with no knowledge of the groups. Results are shown as mean \pm SEM ( $n=3-4) P$ values were determined by two-way ANOVA followed by Dunnett's comparison test, ${ }^{*} P<0.05,{ }^{* *} P<0.01$ compared to mock challenge. 

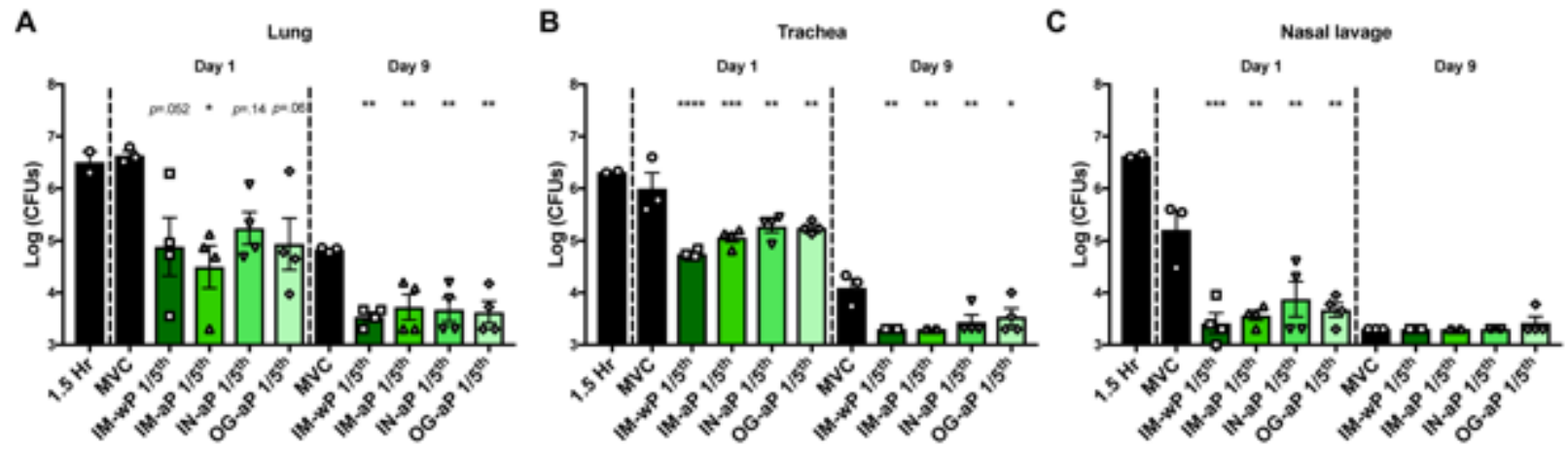

Figure 21. Oral and intranasal immunization decreased $B$. pertussis bacterial burden in the respiratory tract. Bacteria were quantified by serially diluted CFUs following vaccination and intranasal challenge. CFU counts were determined from (A) lung homogenate $(\mathrm{B})$ trachea and $(\mathrm{C})$ nasal lavage $1.5 \mathrm{Hr}, 1-$, and 9-day post $B$. pertussis challenge. Results are shown as mean \pm SEM $(n=2-4)$. $P$ values were determined by one-way ANOVA with Dunnett's post hoc test, ${ }^{*} P<0.05,{ }^{* *} P<0.01,{ }^{* * *} P<0.001$, ${ }^{* * * *} P<0.0001$ compared to mock vac. challenge group. 

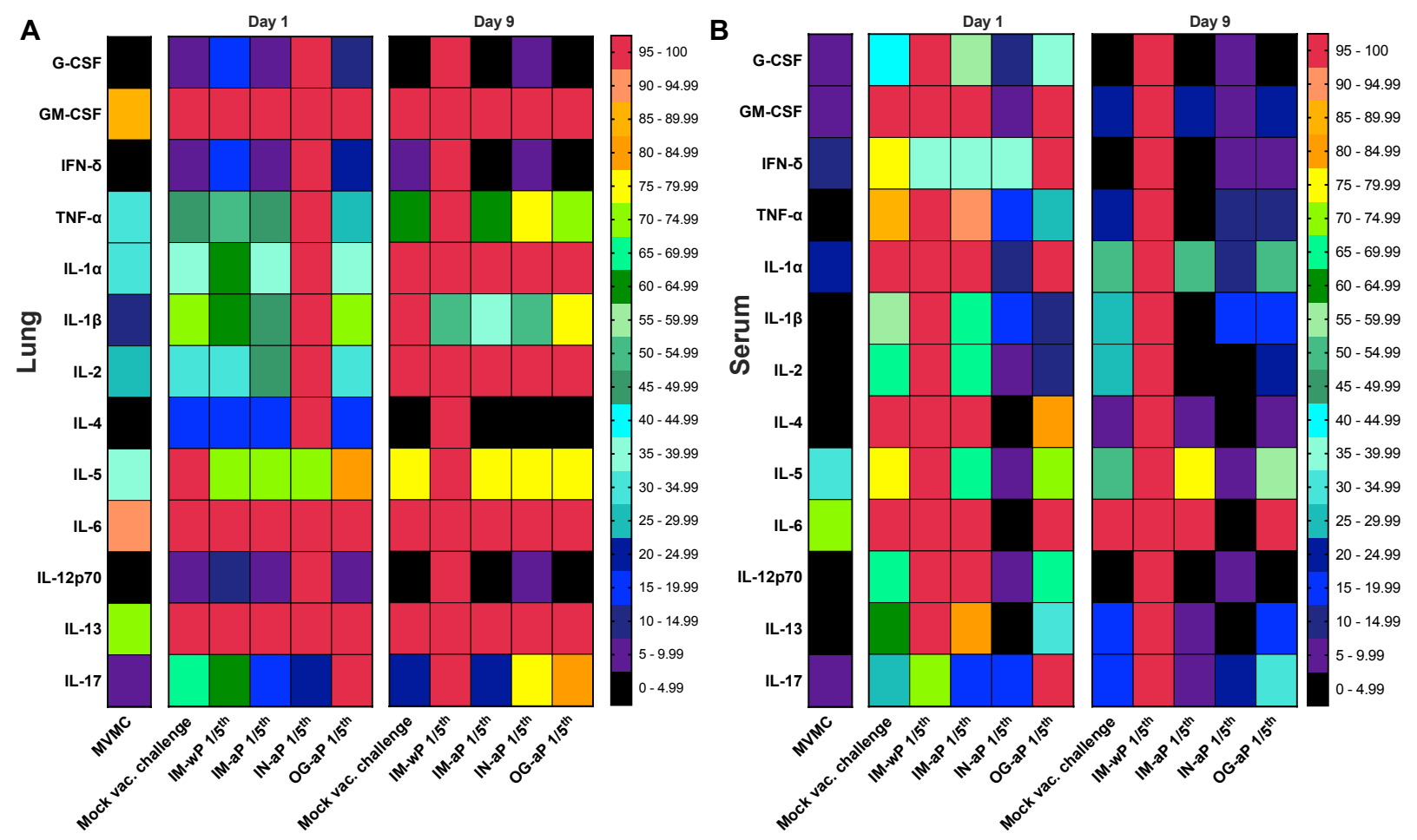

Figure 22. Measurement of cytokines in the lung and serum at days 1 and 9 post infection. Heat map of the average percent cytokines normalized to the max cytokine measured in the $(A)$ lung and $(B)$ serum. MVMC (mock vaccinated mock challenge) cytokines are from rats in (Hall et al 2021). All statistical analysis comparing average cytokine values are in Fig S5-6. 


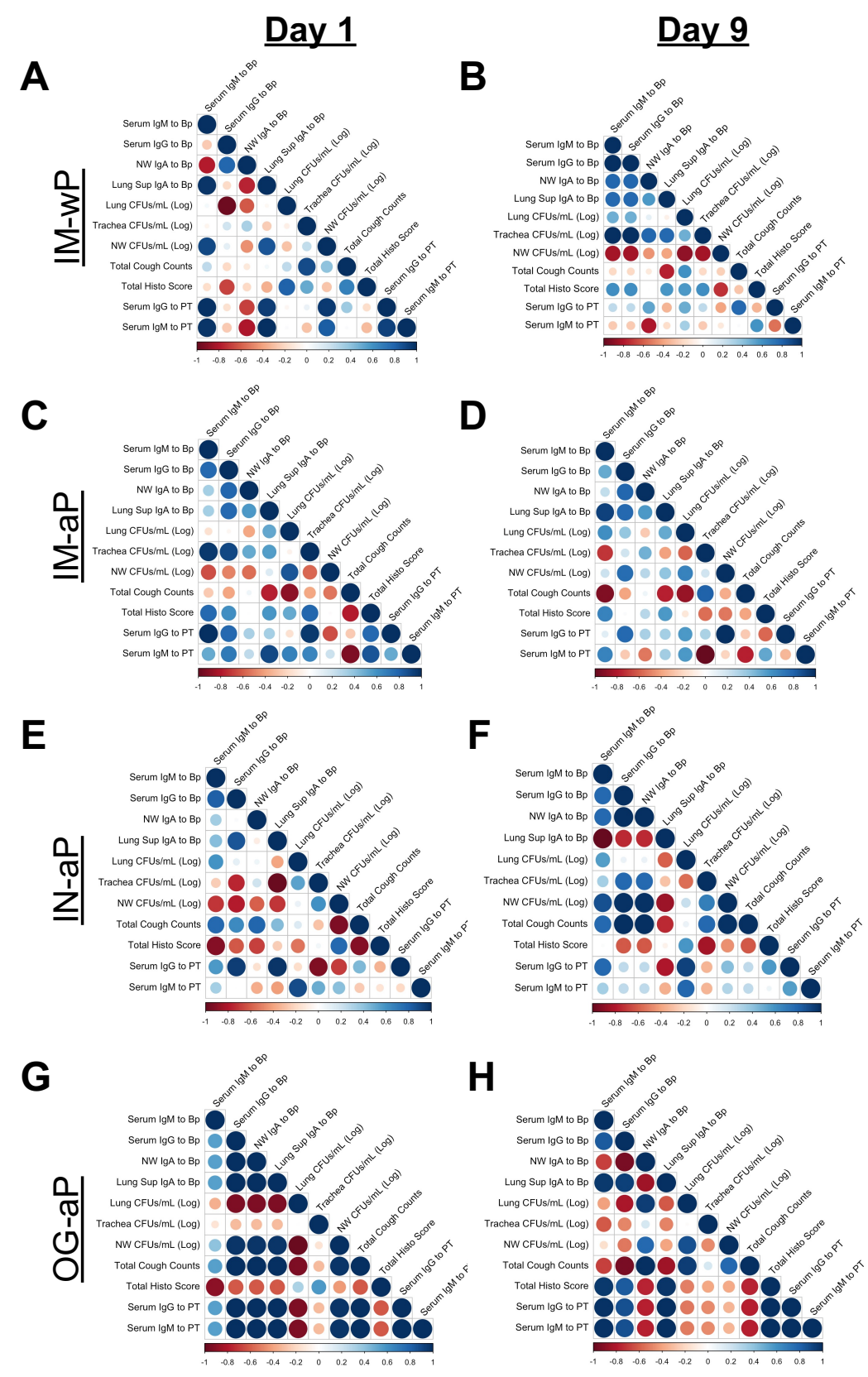

Figure 23. Systemic and mucosal anti-Bp and anti-PT antibodies correlate with observed protection. Correlograms were generated using the observed data for IM-WP (A-B), IMaP (C-D), IN-aP $(E-F)$, and OG-aP $(G-H)$. Program $R$ was used to make correlation graphs from raw data for both day 1 and day 9 post-challenge. $R^{2}$ values were generated when generating the correlograms. Positive correlations are annotated by the blue circles, while the negative correlations are annotated by the red circles. The size of the circle annotates the strength of the correlation. 


\subsection{Supplemental Figures}

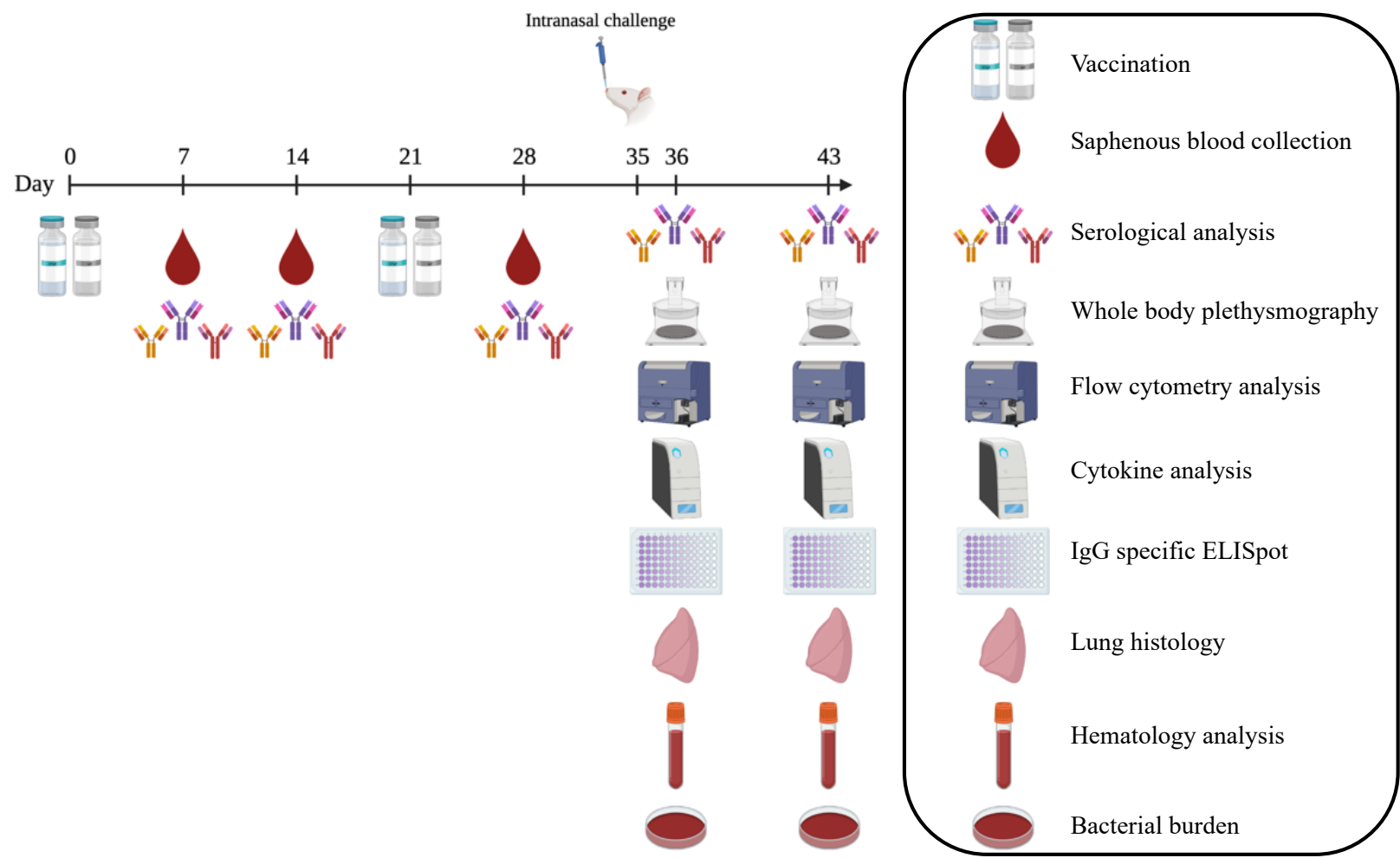

Figure S 13. Experimental design of vaccination and challenge. Three-week old Sprague Dawley rats were vaccinated IM-wP, IM-aP, IN-aP, and OG-aP 1/5 th human dose. 21 days post prime, rats were boosted with the same corresponding vaccine. At days 7,14 , and 28 post prime, blood was collected via saphenous blood draw for serological analysis. At day 35 , rats vaccinated rats were intranasally challenged with $10^{8}$ viable $B p$. Cough and respiratory distress was measured using whole body plethysmography for 8 days post challenge. Antibody titers in the serum, lung, and nasal cavity was measured at days 1 and 9 post challenge. Flow cytometry was used to measure neutrophil and $b$ cell recruitment in the lung and blood at days 1 and 9 post challenge. The left lobe of the lung was sectioned and stained with H\&E for analysis of inflammation at days 1 and 9 post challenge. Antigen specific IgG ELISpot assay was performed to measure antigen specific $B$ cells in the bone marrow at days 1 and 9 post challenge. Cytokines in the lung and serum were measured post challenge. Hematology was used to measure blood cell populations post challenge. Bacterial burden in the respiratory tract was measured at days 1 and 9 post challenge. Created with Biorender.com 
A
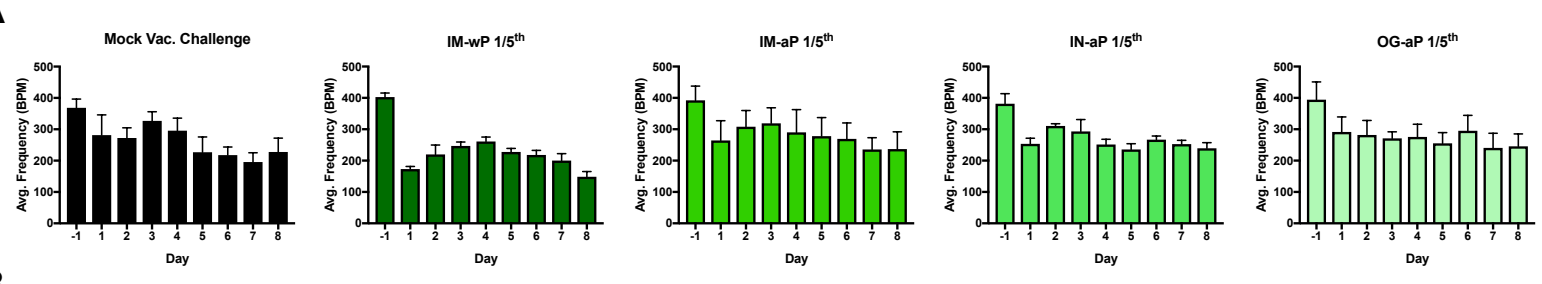

B
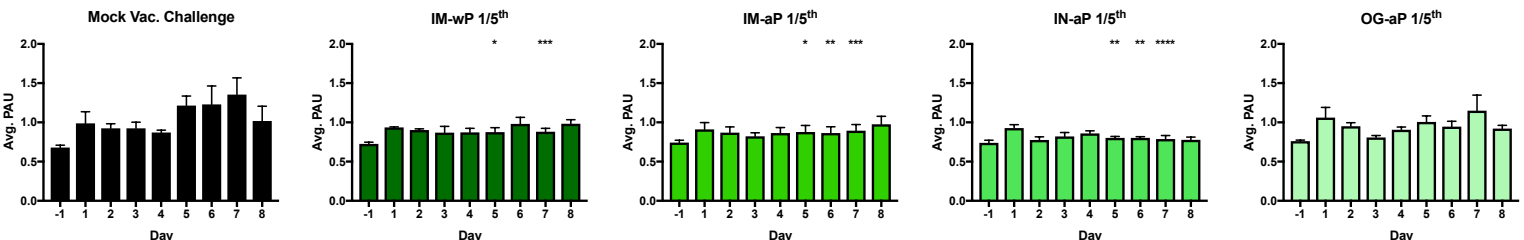

C
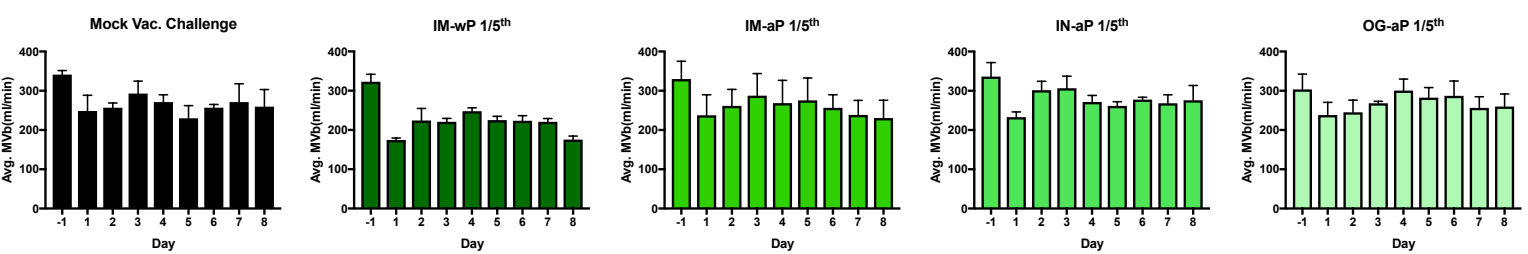

D
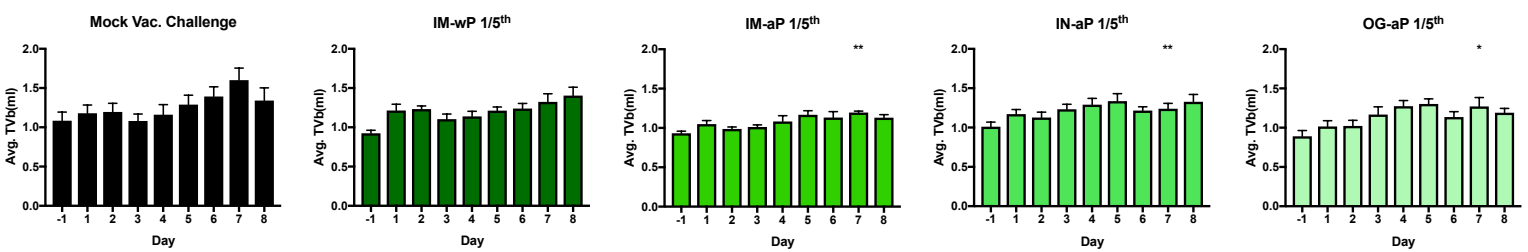

E
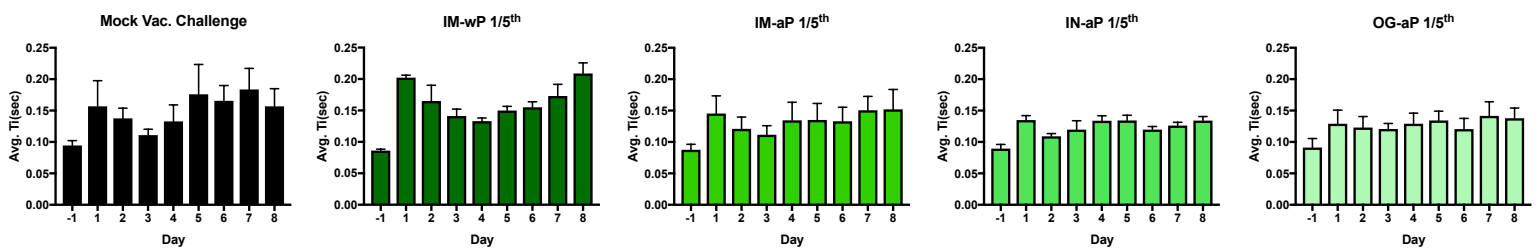

$\mathbf{F}$
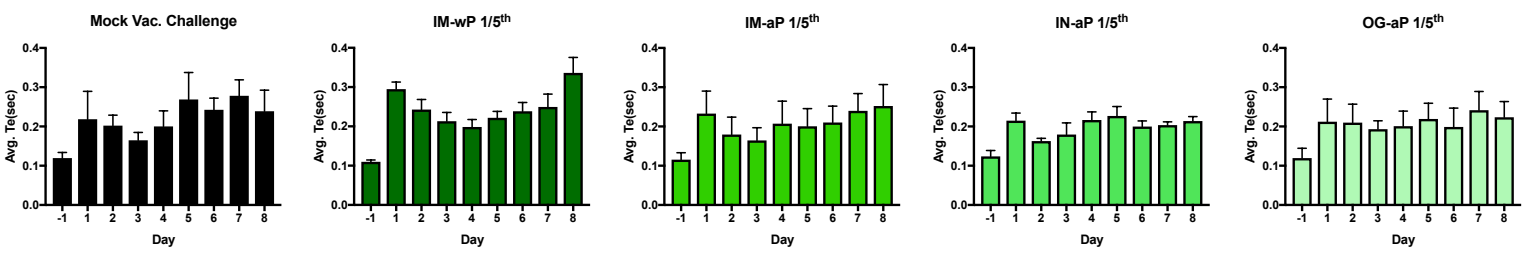

Figure S 14. Respiratory profile of Sprague-Dawley rats vaccinated and challenged with B. pertussis. Every day after 5 p.m. whole body plethysmography was used to analyze the respiratory capacity of immunized and challenge rats. Measurements assessed were: (A) frequency of breathes, (B) pause (PAU), (C) minute volume (MVb), (D) tidal volume (TVb), (E) time of inspiration $(\mathrm{Ti})$, and $(\mathrm{F})$ time of expiration $(\mathrm{Te})$. Results shown as mean $\pm \mathrm{SEM}$ $(n=3-4)$. $P$ values were determined by two-way ANOVA with Dunnett's post hoc test, 
${ }^{*} P<0.05,{ }^{* *} P<0.01,{ }^{* * *} P<0.001$, and ${ }^{* * * *} P<0.0001$ compared to mock vac. challenge group. 


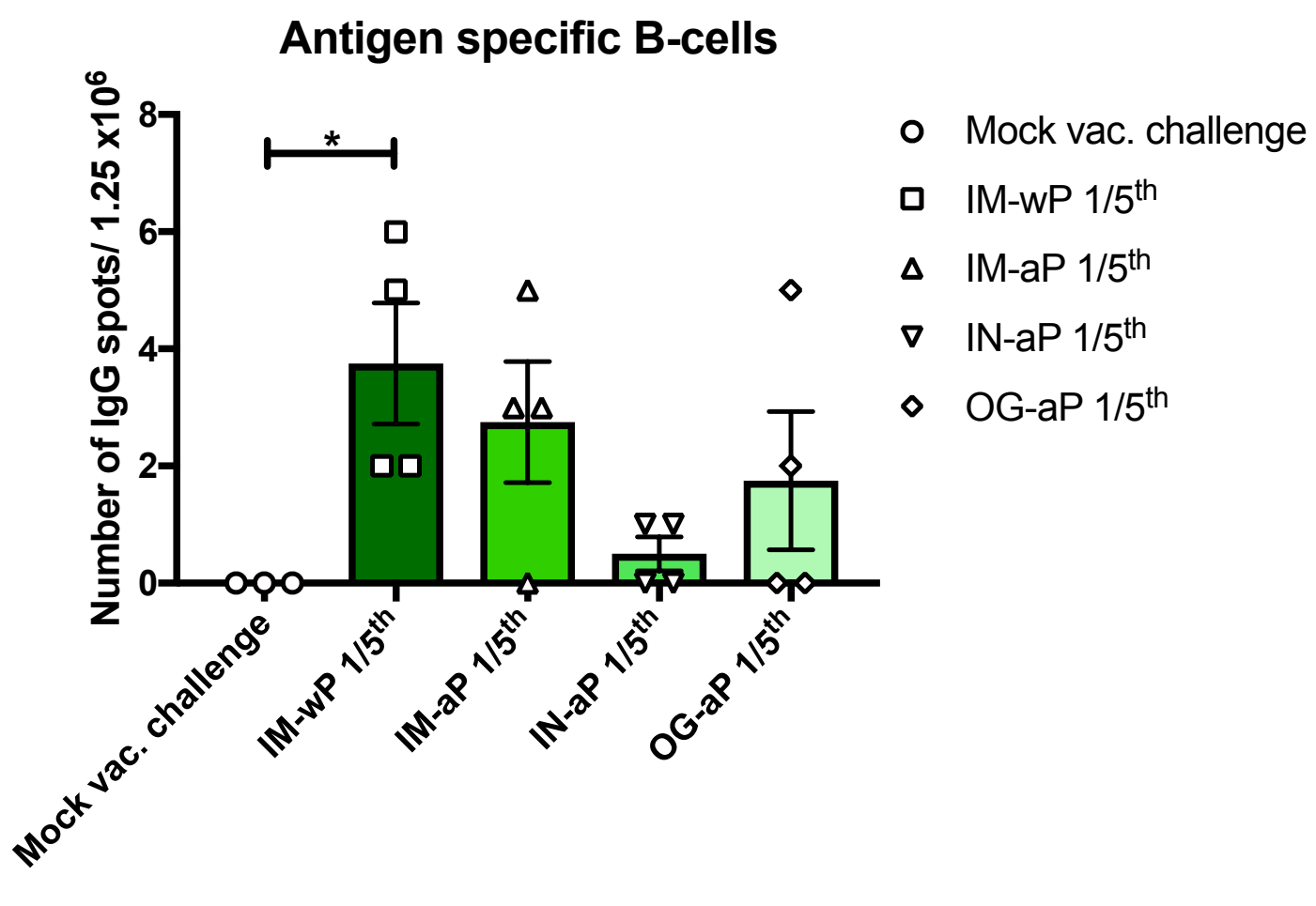

Figure S 15. ELISpot assay was performed to measure the number of $B p$ specific IgG cells from the bone marrow at day 9 post challenge. Results are shown as mean \pm SEM $(n=3-4)$. $P$ values were determined by one-way ANOVA with Dunnett's post hoc test, ${ }^{*} P<0.05$ compared to mock vaccinated challenge group. 
A

Lung weight

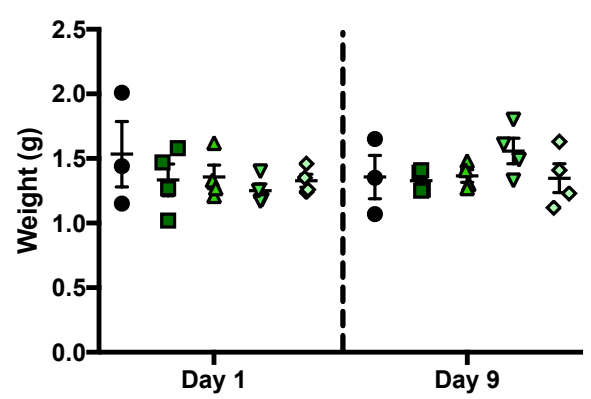

B

Percent weight change

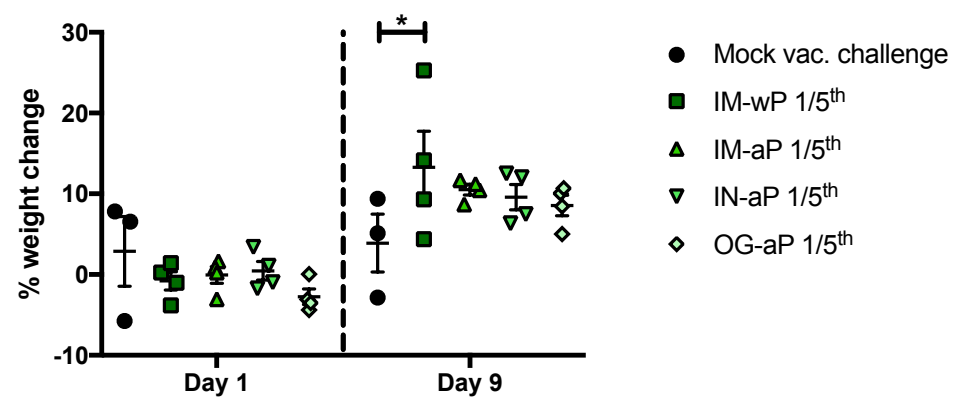

Figure $\mathbf{S}$ 16. Measurement of lung and body weight at days 1 and 9 post challenge. (A) Lung weight as measured immediately after euthanasia before being homogenized. Body weight for each rat was measured before challenge and immediately post euthanasia. (B) Percent weight change was calculated by taking the differences between starting weight and end weight and dividing by the initial weight multiplying by 100 . Results shown as mean \pm SEM $(n=3-4)$. $P$ values were determined by two-way ANOVA with Dunnett's post hoc test, ${ }^{*} P<0.05$ compared to the mock vaccinated challenge group. 
A
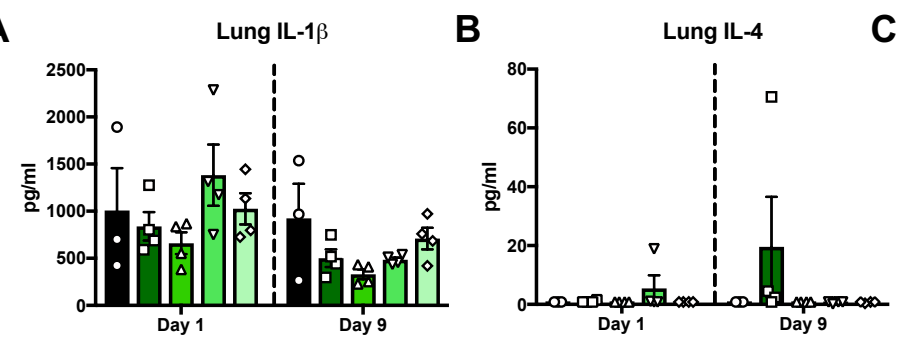

D

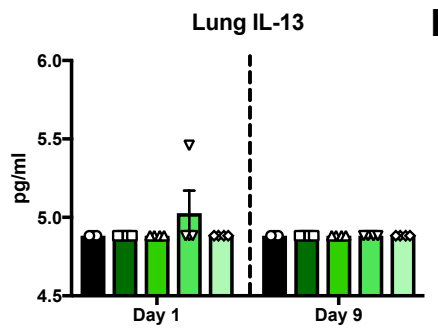

E

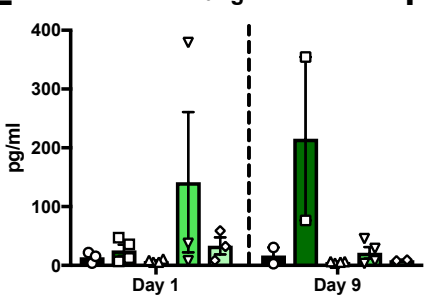

$\mathbf{F}$

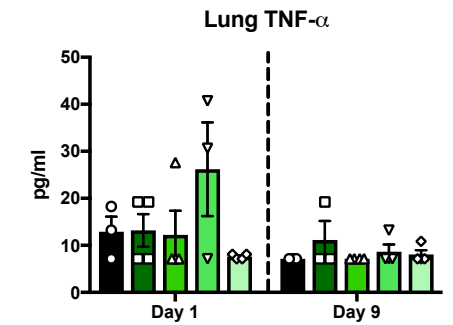

- Mock vac. challenge

口 IM-wP $1 / 5^{\text {th }}$

$\Delta \quad \mathrm{IM}-\mathrm{aP} 1 / 5^{\text {th }}$

$\nabla$ IN-aP $1 / 5^{\text {th }}$

$\diamond$ OG-aP $1 / 5^{\text {th }}$
G

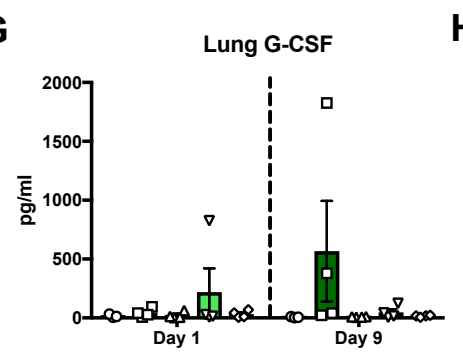

H

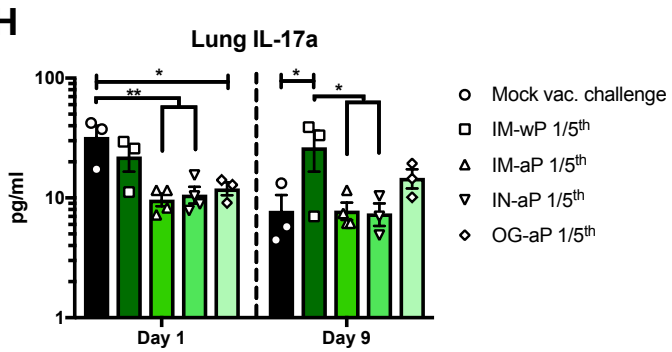

Figure $\mathbf{S}$ 17. Measurement of cytokines in the lung at days 1 and 9 post infection. Cytokines in the lung supernatant were analyzed using ProcartaPlex multiplex immunoassay kit. Results shown as mean \pm SEM $(n=3-4)$. $P$ values were determined by two-way ANOVA with Dunnett's post hoc test, ${ }^{*} P<0.05, "{ }^{\prime \prime} P<0.01$ compared between mock vaccinated challenge and vaccinated challenge groups. 
A
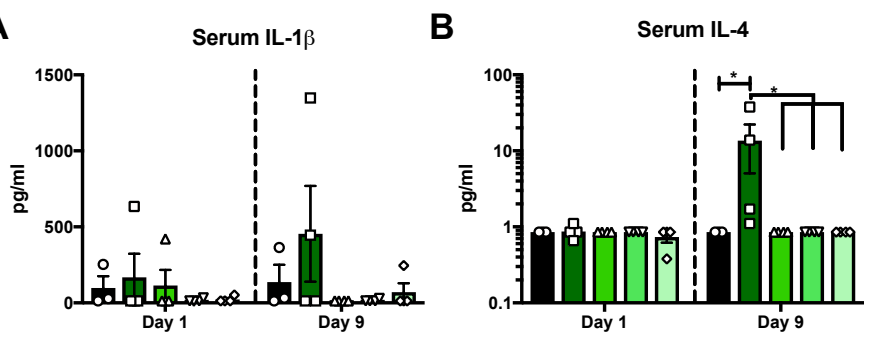

D

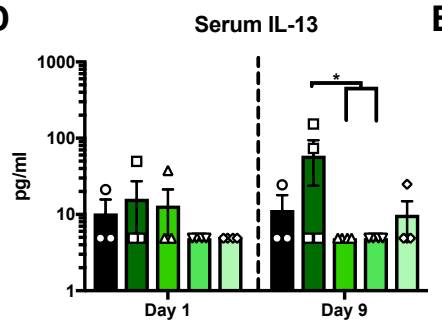

E

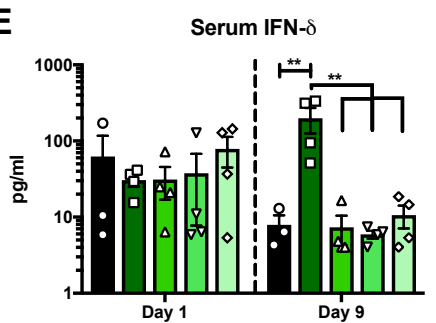

G

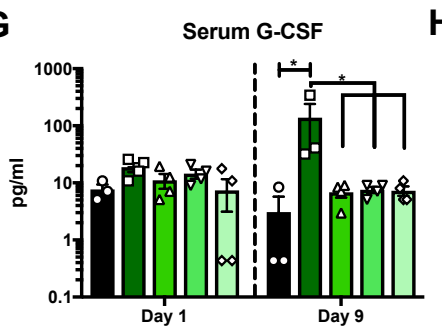

H

Serum IL-17a

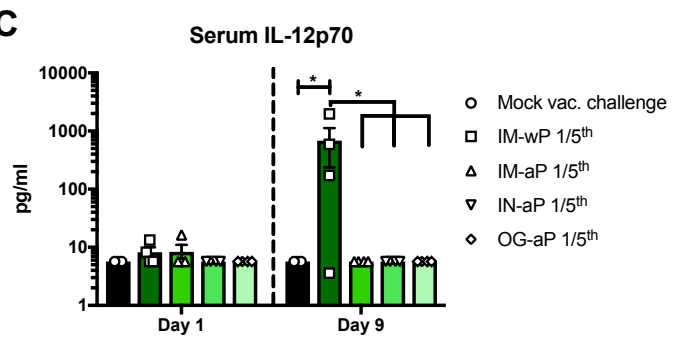

$\mathbf{F}$

Serum TNF- $\alpha$
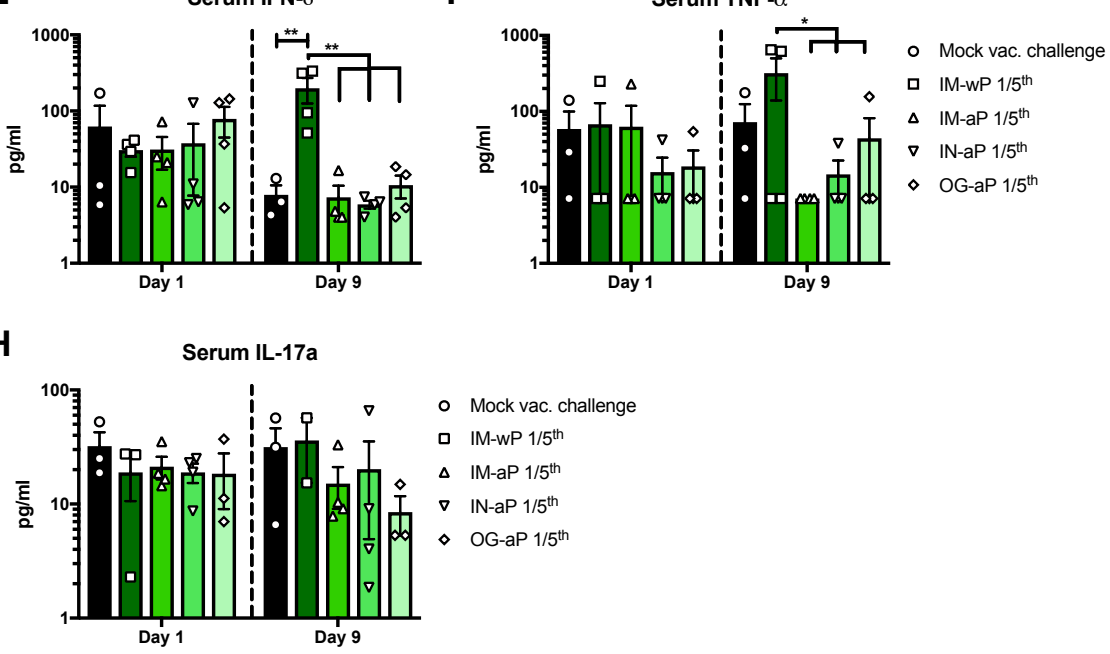

Figure $\mathbf{S}$ 18. Measurement of cytokines in the serum at days 1 and 9 post infection. Cytokines in the serum and lung supernatant were analyzed using ProcartaPlex multiplex immunoassay kit. Results shown as mean \pm SEM $(n=3-4)$. $P$ values were determined by two-way ANOVA with Dunnett's post hoc test, ${ }^{*} P<0.05,{ }^{* *} P<0.01$ compared between mock vaccinated challenge and vaccinated challenge groups. 
A

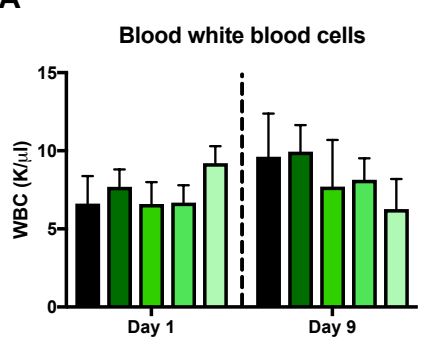

D

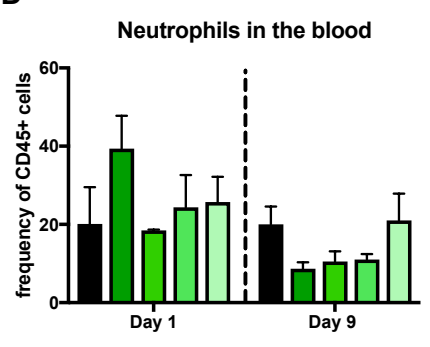

B

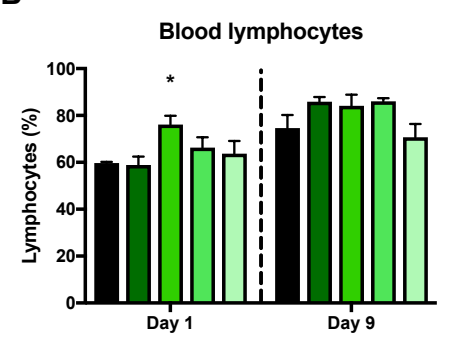

E

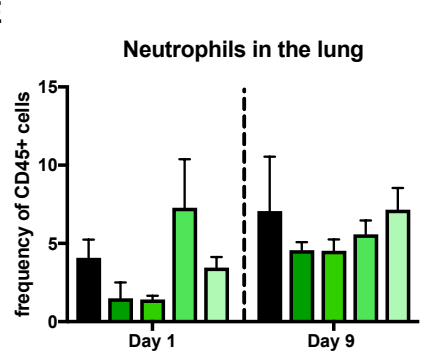

C

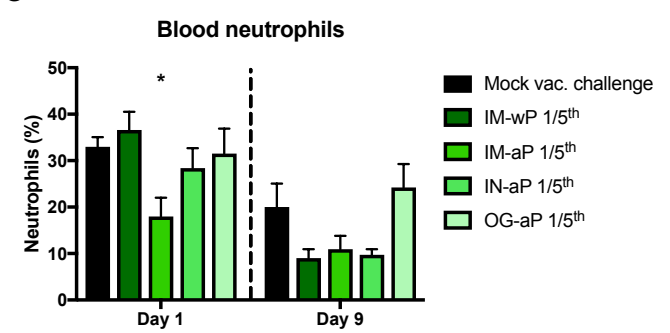

$\mathbf{F}$

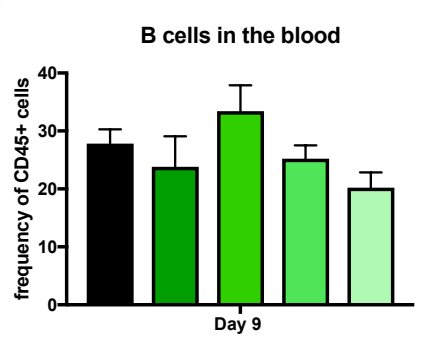

Mock vac. challenge

$\square$ IM-wP $1 / 5^{\text {th }}$ $\square \mathrm{IM}-\mathrm{aP} 1 / 5^{\text {th }}$ $\square \mathrm{IN}-\mathrm{aP} 1 / 5^{\text {th }}$ $\square$ OG-aP $1 / 5^{\text {th }}$

Figure S 19. Characterizing circulating cell populations in the blood and lung post infection. ProCyte hematology analyzer was used to assess (A) total white blood cells, (B) percent lymphocytes and (C) percent neutrophils in the blood post challenge. Flowcytometry analysis of circulating $B$ cells and neutrophils in the lung and blood. (D\&E) Neutrophils were gated by $\left(C D 45^{+}\right.$CD161- B220- CD43+ His48 $\left.{ }^{\text {hi }}\right)$, while $(F)$ B cells were gate by $\left(C D 45^{+} C D 45 R^{+}\right)$. Neutrophil and $B$ cell quantification represented as percentage of single, CD45+ cells. Results are shown as mean \pm SEM $(n=3-4)$. $P$ values were determined by two-way ANOVA followed by Dunnett's comparison test, ${ }^{*} P<0.05$, compared between mock vaccinated challenge group. 


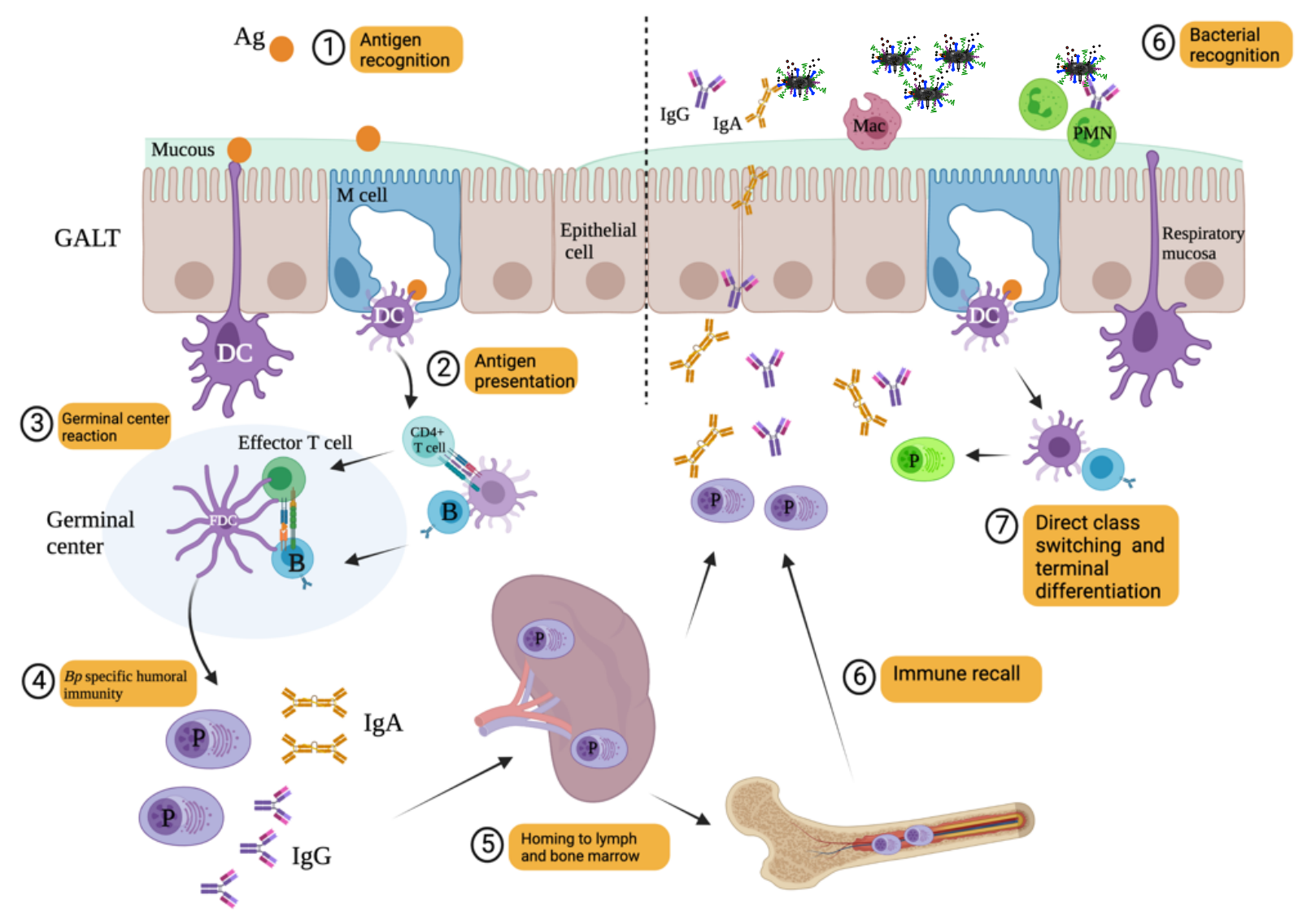

Figure S 20. Proposed mucosal immune response induced through oral vaccination. Upon oral vaccination of DTaP, antigens travel to the gut associated lymphoid tissue. Here, antigens are endocytosed by M cells or phagocytosed by DCs in the epithelial layer and antigens are presented to $T$ and $B$ cells in the lamina propria. Ensuing germinal center reaction occur in lymphoid follicles and induce $B p$ specific plasma cells capable of producing IgG and IgA antibodies. Generated plasma cells can home to secondary lymphoid tissue and travel to the bone marrow awaiting recall. Upon $B p$ challenge, plasma cells are recalled and migrate to the respiratory mucosa secreting $\lg G$ and $\lg A$ antibodies. Here antibodies can opsonize the bacteria and potentially play a role in antibody mediated phagocytosis of the bacteria with recruited neutrophils and macrophages. Other potential mechanism for the generation of antibody secreting plasma cells involves direct class switching and terminal differentiation. 


\subsection{References}

1. Bordet J, Gengou O. 1906. Le Microbe de la Coqueluche. Les Ann l'Institut Pasteur 20:731-741.

2. Mattoo S, Cherry JD. 2005. Molecular pathogenesis, epidemiology, and clinical manifestations of respiratory infections due to Bordetella pertussis and other Bordetella subspecies. Clin Microbiol Rev 18:326-382.

3. Melvin JA, Scheller E V., Miller JF, Cotter PA. 2014. Bordetella pertussis pathogenesis: Current and future challenges. Nat Rev Microbiol2014/03/13. $12: 274-288$.

4. Kapil P, Merkel TJ. 2019. Pertussis vaccines and protective immunity. Curr Opin Immunol 59:72-78.

5. CDC, Ncird. Immunology and Vaccine-Preventable Diseases - Pink Book Pertussis.

6. Pittman M. 1991. History of the development of pertussis vaccine. Dev Biol Stand1991/01/01. 73:13-29.

7. Hill Elam-Evans LD, Yankey D, Singleton JA, Kang Y. HA. 2017. Vaccination Coverage Among Children Aged 19-35 Months — United States, 2017. MMWR Morb Mortal Wkly Rep 2018 1123-1128.

8. Yeung KHT, Duclos P, Nelson EAS, Hutubessy RCW. 2017. An update of the global burden of pertussis in children younger than 5 years: a modelling study. Lancet Infect Dis 17:974-980.

9. Klein NP, Bartlett J, Fireman B, Baxter R. 2016. Waning Tdap Effectiveness in Adolescents. Pediatrics 137:e20153326-e20153326.

10. Klein NP, Bartlett J, Rowhani-Rahbar A, Fireman B, Baxter R. 2012. Waning 
Protection after Fifth Dose of Acellular Pertussis Vaccine in Children. N Engl J Med 367:1012-1019.

11. Klein NP, Bartlett J, Fireman B, Rowhani-Rahbar A, Baxter R. 2013. Comparative Effectiveness of Acellular Versus Whole-Cell Pertussis Vaccines in Teenagers. Pediatrics 131:e1716-e1722.

12. Althouse BM, Scarpino S V. 2015. Asymptomatic transmission and the resurgence of Bordetella pertussis. BMC Med 13:146.

13. Templeton KE, Scheltinga SA, van der Zee A, Diederen BMW, van Kruijssen AM, Goossens H, Kuijper E, Claas ECJ. 2003. Evaluation of real-time PCR for detection of and discrimination between Bordetella pertussis, Bordetella parapertussis, and Bordetella holmesii for clinical diagnosis. J Clin Microbiol $41: 4121-6$.

14. Warfel JM, Zimmerman LI, Merkel TJ. 2014. Acellular pertussis vaccines protect against disease but fail to prevent infection and transmission ina nonhuman primate model. Proc Natl Acad Sci U S A 111:787-792.

15. Wendelboe AM, Van Rie A, Salmaso S, Englund JA. 2005. Duration of Immunity Against Pertussis After Natural Infection or Vaccination. Pediatr Infect Dis J 24:S58-S61.

16. Wilk MM, Misiak A, McManus RM, Allen AC, Lynch MA, Mills KHG. 2017. Lung CD4 Tissue-Resident Memory T Cells Mediate Adaptive Immunity Induced by Previous Infection of Mice with Bordetella pertussis. J Immunol 199:233-243.

17. Chen K, Magri G, Grasset EK, Cerutti A. 2020. Rethinking mucosal antibody responses: IgM, IgG and IgD join IgA. Nat Rev Immunol. Nature Research. 
18. Goodman YE, Wort AJ, Jackson FL. 1981. Enzyme-linked immunosorbent assay for detection of pertussis immunoglobulin $A$ in nasopharyngeal secretions as an indicator of recent infection. J Clin Microbiol 13:286-292.

19. Tuomanen El, Zapiain LA, Galvan P, Hewlett EL. 1984. Characterization of antibody inhibiting adherence of Bordetella pertussis to human respiratory epithelial cells. J Clin Microbiol 20:167.

20. Hellwig SMM, Van Spriel AB, Schellekens JFP, Mooi FR, Van de Winkel JGJ. 2001. Immunoglobulin A-mediated protection against Bordetella pertussis infection. Infect Immun 69:4846-4850.

21. Thomas MG, Redhead K, Lambert HP. 1989. Human Serum Antibody Responses to Bordetella pertussis Infection and Pertussis Vaccination. J Infect Dis 159:211218.

22. Marcellini V, Piano Mortari E, Fedele G, Gesualdo F, Pandolfi E, Midulla F, Leone P, Stefanelli P, Tozzi AE, Carsetti R. 2017. Protection against Pertussis in Humans Correlates to Elevated Serum Antibodies and Memory B Cells. Front Immunol 8:1158.

23. Mills KH, Barnard A, Watkins J, Redhead K. 1993. Cell-mediated immunity to Bordetella pertussis: role of Th1 cells in bacterial clearance in a murine respiratory infection model. Infect Immun 61:399-410.

24. Dunne A, Ross PJ, Pospisilova E, Masin J, Meaney A, Sutton CE, Iwakura Y, Tschopp J, Sebo P, Mills KHG. 2010. Inflammasome Activation by Adenylate Cyclase Toxin Directs Th17 Responses and Protection against Bordetella pertussis. J Immunol 185:1711-1719. 
25. Warfel JM, Merkel TJ. 2013. Bordetella pertussis infection induces a mucosal IL17 response and long-lived Th17 and Th1 immune memory cells in nonhuman primates. Mucosal Immunol 6:787-796.

26. Solans L, Locht C. 2019. The role of mucosal immunity in pertussis. Front Immunol. Frontiers Media S.A.

27. Boehm DT, Wolf MA, Hall JM, Wong TY, Sen-Kilic E, Basinger HD, Dziadowicz SA, Gutierrez M de la P, Blackwood CB, Bradford SD, Begley KA, Witt WT, Varney ME, Barbier M, Damron FH. 2019. Intranasal acellular pertussis vaccine provides mucosal immunity and protects mice from Bordetella pertussis. npj Vaccines 4 .

28. Wolf MA, Boehm DT, DeJong MA, Wong TY, Sen-Kilic E, Hall JM, Blackwood CB, Weaver KL, Kelly CO, Kisamore CA, Bitzer GJ, Bevere JR, Barbier M, Damron FH. 2020. Intranasal immunization with acellular pertussis vaccines results in long-term immunity to Bordetella pertussis in mice . Infect Immun IAI.00607-20.

29. Maurer H, Höfler K, Hilbe W, Huber E. 1979. Preliminary findings with oral whooping cough vaccination in young infants. Wien Hlin Wochenschr.

30. Baumann E, Binder BR, Falk W, Huber EG, Kurz R, Rosanelli K. 1985. Development and clinical use of an oral heat-inactivated whole cell pertussis vaccine. Dev Biol Stand 61:511-6.

31. Guzman CA, Brownlie RM, Kadurugamuwa J, Walker MJ, Timmis KN. 1991. Antibody responses in the lungs of mice following oral immunization with Salmonella typhimurium aroA and invasive Escherichia coli strains expressing the filamentous hemagglutinin of Bordetella pertussis. Infect Immun 59:4391-4397. 
32. Lim A, Ng JKW, Locht C, Alonso S. 2014. Protective role of adenylate cyclase in the context of a live pertussis vaccine candidate. Microbes Infect 16:51-60.

33. Skerry CM, Mahon BP. 2011. A live, attenuated Bordetella pertussis vaccine provides long-term protection against virulent challenge in a murine model. Clin Vaccine Immunol2010/12/08. 18:187-193.

34. Thorstensson R, Trollfors B, Al-Tawil N, Jahnmatz M, Bergström J, Ljungman M, Törner A, Wehlin L, Van Broekhoven A, Bosman F, Debrie AS, Mielcarek N, Locht C. 2014. A phase I clinical study of a live attenuated Bordetella pertussis vaccine BPZE1; a single centre, double-blind, placebo-controlled, dose-escalating study of BPZE1 given intranasally to healthy adult male volunteers. PLoS One 9:e83449.

35. Lin A, Apostolovic D, Jahnmatz M, Liang F, Ols S, Tecleab T, Wu C, van Hage M, Solovay K, Rubin K, Locht C, Thorstensson R, Thalen M, Loré K. 2020. Live attenuated pertussis vaccine BPZE1 induces a broad antibody response in humans. J Clin Invest 130:2332-2346.

36. Hornibrook JW, Ashburn LL. 1939. A Study of Experimental Pertussis in the Young Rat. Public Heal Reports 54:439.

37. Woods DE, Franklin R, Cryz SJ, Ganss M, Peppler M, Ewanowich C. 1989. Development of a rat model for respiratory infection with Bordetella pertussis. Infect Immun 57:1018-1024.

38. Hall E, Parton R, Wardlaw AC. 1994. Cough production, leucocytosis and serology of rats infected intrabronchially with Bordetella pertussis. J Med Microbiol 40:205-213.

39. Parton R, Hall E, Wardlaw AC. 1994. Responses to Bordetella pertussis mutant 
strains and to vaccination in the coughing rat model of pertussis. J Med Microbiol 40:307-312.

40. Hall E, Parton R, Wardlaw AC. 1997. Differences in coughing and other responses to intrabronchial infection with Bordetella pertussis among strains of rats. Infect Immun 65:4711-4717.

41. Hall E, Parton R, Wardlaw AC. 1998. Responses to acellular pertussis vaccines and component antigens in a coughing-rat model of pertussis. Vaccine 16:1595603.

42. Hall E, Parton R, Wardlaw AC. 1999. Time-course of infection and responses in a coughing rat model of pertussis. J Med Microbiol 48:95-98.

43. Hall JM, Kang J, Kenney SM, Wong TY, Bitzer GJ, Kelly CO, Kisamore CA, Boehm DT, DeJong MA, Allison M, Sen-Kilic E, Horspool AM, Bevere JR, Barbier M, Heath Damron F. 2021. Re-investigating the coughing rat model of pertussis to understand Bordetella pertussis pathogenesis . bioRxiv 2021.04.02.438291.

44. Ross PJ, Sutton CE, Higgins S, Allen AC, Walsh K, Misiak A, Lavelle EC, McLoughlin RM, Mills KHG. 2013. Relative Contribution of Th1 and Th17 Cells in Adaptive Immunity to Bordetella pertussis: Towards the Rational Design of an Improved Acellular Pertussis Vaccine. PLoS Pathog 9:e1003264.

45. Ryan M, Murphy G, Ryan E, Nilsson L, Shackley F, Gothefors L, Øymar K, Miller E, Storsaeter J, Mills KH. 1998. Distinct T-cell subtypes induced with whole cell and acellular pertussis vaccines in children. Immunology 93:1-10.

46. Ausiello CM, Urbani F, la Sala A, Lande R, Cassone A. 1997. Vaccine- and antigen-dependent type 1 and type 2 cytokine induction after primary vaccination 
of infants with whole-cell or acellular pertussis vaccines. Infect Immun 65:21682174.

47. Mahon BP, Sheahan BJ, Griffin F, Murphy G, Mills KH. 1997. Atypical disease after Bordetella pertussis respiratory infection of mice with targeted disruptions of interferon-gamma receptor or immunoglobulin mu chain genes. J Exp Med $186: 1843-51$.

48. Barbic J, Leef MF, Burns DL, Shahin RD. 1997. Role of gamma interferon in natural clearance of Bordetella pertussis infection. Infect Immun 65:4904-4908.

49. Mills KHG, Ryan M, Ryan E, Mahon BP. 1998. A murine model in which protection correlates with pertussis vaccine efficacy in children reveals complementary roles for humoral and cell- mediated immunity in protection against Bordetella pertussis. Infect Immun 66:594-602.

50. Redhead K, Watkins J, Barnard A, Mills KHG. 1993. Effective immunization against Bordetella pertussis respiratory infection in mice is dependent on induction of cell-mediated immunity. Infect Immun 61:3190-3198.

51. Sawal M, Cohen M, Irazuzta JE, Kumar R, Kirton C, Brundler M-A, Evans CA, Wilson JA, Raffeeq P, Azaz A, Rotta AT, Vora A, Vohra A, Abboud P, Mirkin LD, Cooper M, Dishop MK, Graf JM, Petros A, Klonin H. 2009. Fulminant pertussis: A multi-center study with new insights into the clinico-pathological mechanisms. Pediatr Pulmonol 44:970-980.

52. Paddock CD, Sanden GN, Cherry JD, Gal AA, Langston C, Tatti KM, Wu K, Goldsmith CS, Greer PW, Montague JL, Eliason MT, Holman RC, Guarner J, Shieh W, Zaki SR. 2008. Pathology and Pathogenesis of Fatal Bordetella 
pertussis Infection in Infants. Clin Infect Dis 47:328-338.

53. Morse SI, Morse JH. 1976. Isolation and properties of the leukocytosis- and lymphocytosis- promoting factor of Bordetella pertussis. J Exp Med 143:14831502.

54. MORSE SI. 1965. STUDIES ON THE LYMPHOCYTOSIS INDUCED IN MICE BY BORDETELLA PERTUSSIS. J Exp Med 121:49-68.

55. Morse SI, Riester SK. 1967. Studies on the leukocytosis and lymphocytosis induced by Bordetella pertussis. I. Radioautographic analysis of the circulating cells in mice undergoing pertussis-induced hyperleukocytosis. J Exp Med 125:401-8.

56. Heininger U, Klich K, Stehr K, Cherry JD. 1997. Clinical findings in Bordetella pertussis infections: results of a prospective multicenter surveillance study. Pediatrics 100:E10.

57. Blackwood CB, Sen-Kilic E, Boehm DT, Hall JM, Varney ME, Wong TY, Bradford SD, Bevere JR, Witt WT, Damron FH, Barbier M. 2020. Innate and adaptive immune responses against Bordetella pertussis and Pseudomonas aeruginosa in a murine model of mucosal vaccination against respiratory infection. Vaccines $8: 1-21$.

58. Correlogram. https://www.r-graph-gallery.com/correlogram.html

59. Bewick V, Cheek L, Ball J. 2003. Statistics review 7: Correlation and regression. Crit Care. BioMed Central.

60. Althouse BM, Scarpino S V. 2015. Asymptomatic transmission and the resurgence of Bordetella pertussis https://doi.org/10.1186/s12916-015-0382-8. 
61. Klein NP, Bartlett J, Fireman B, Aukes L, Buck PO, Krishnarajah G, Baxter R. 2017. Waning protection following 5 doses of a 3-component diphtheria, tetanus, and acellular pertussis vaccine. Vaccine 35:3395-3400.

62. Nakamura K, Shinoda N, Hiramatsu Y, Ohnishi S, Kamitani S, Ogura Y, Hayashi T, Horiguchi Y. 2019. BspR/BtrA, an Anti- $\sigma$ Factor, Regulates the Ability of Bordetella bronchiseptica To Cause Cough in Rats. mSphere https://doi.org/10.1128/msphere.00093-19.

63. Boehm DT, Hall JM, Wong TY, DiVenere A, Sen-Kilic E, Bevere JR, Bradford SD, Blackwood CB, Elkins C, DeRoos KA, Gray MC, Cooper CG, Varney ME, Maynard JA, Hewlett EL, Barbier M, Damron FH. 2018. Evaluation of adenylate cyclase toxoid antigen in acellular pertussis vaccines using a Bordetella pertussis challenge model in mice. Infect Immun IAI.00857-17.

64. Shi W, Kou Y, Jiang H, Gao F, Kong W, Su W, Xu F, Jiang C. 2018. Novel intranasal pertussis vaccine based on bacterium-like particles as a mucosal adjuvant. Immunol Lett 198:26-32.

65. Allen AC, Wilk MM, Misiak A, Borkner L, Murphy D, Mills KHG. 2018. Sustained protective immunity against Bordetella pertussis nasal colonization by intranasal immunization with a vaccine-adjuvant combination that induces IL-17-secreting T RM cells. Mucosal Immunol 11:1763-1776.

66. Ryan EJ, Mcneela E, Murphy GA, Stewart H, O’Hagan D, Pizza M, Rappuoli R, Mills KHG. 1999. Mutants of Escherichia coli heat-labile toxin act as effective mucosal adjuvants for nasal delivery of an acellular pertussis vaccine: Differential effects of the nontoxic AB complex and enzyme activity on Th1 and Th2 cells. 
Infect Immun 67:6270-6280.

67. Locht C, Papin JF, Lecher S, Debrie A-S, Thalen M, Solovay K, Rubin K, Mielcarek N. 2017. Live Attenuated Pertussis Vaccine BPZE1 Protects Baboons Against Bordetella pertussis Disease and Infection. J Infect Dis2017/05/23. 216:117-124.

68. Solans L, Debrie A-S, Borkner L, Aguiló N, Thiriard A, Coutte L, Uranga S, Trottein F, Martín C, Mills KHG, Locht C. 2018. IL-17-dependent SlgA-mediated protection against nasal Bordetella pertussis infection by live attenuated BPZE1 vaccine. Mucosal Immunol 11:1753-1762.

69. Lin A, Apostolovic D, Jahnmatz M, Liang F, Ols S, Tecleab T, Wu C, van Hage M, Solovay K, Rubin K, Locht C, Thorstensson R, Thalen M, Loré K. 2020. Live attenuated pertussis vaccine BPZE1 induces a broad antibody response in humans. J Clin Invest 130.

70. Baumann E, Binder BR, Falk W. 1985. Development and clinical use of an oral heat-inactivated whole cell pertussis vaccine. Dev Biol Stand VOL. 61:511-516.

71. Strugnell R, Dougan G, Chatfield S, Charles I, Fairweather N, Tite J, Li JL, Beesley J, Roberts M. 1992. Characterization of a Salmonella typhimurium aro vaccine strain expressing the P.69 antigen of Bordetella pertussis. Infect Immun 60.

72. Molina NC, Parker CD. 1990. Murine antibody response to oral infection with live aroA recombinant Salmonella dublin vaccine strains expressing filamentous hemagglutinin antigen from Bordetella pertussis. Infect Immun 58.

73. Plotkin SA. 2010. Correlates of protection induced by vaccination. Clin Vaccine 
Immunol. American Society for Microbiology.

74. Murphy TV, Slade BA, Broder KR, Kretsinger K, Tiwari T, Joyce PM I, JK, Brown K MJAC on IP, Prevention. C for DC and. 2008. Prevention of Pertussis, Tetanus, and Diphtheria Among Pregnant and Postpartum Women and their Infants Recommendations of the Advisory Committee on Immunization Practices (ACIP). MMWR Recomm Reports 1-51.

75. Frizzell H, Woodrow KA. 2020. Biomaterial Approaches for Understanding and Overcoming Immunological Barriers to Effective Oral Vaccinations. Adv Funct Mater 30:1907170.

76. Azizi A, Kumar A, Diaz-Mitoma F, Mestecky J. 2010. Enhancing oral vaccine potency by targeting intestinal M cells. PLoS Pathog. Public Library of Science.

77. Kilgore PE, Salim AM, Zervos MJ, Schmitt H-J. 2016. Pertussis: Microbiology, Disease, Treatment, and Prevention. Clin Microbiol Rev 29:449-86.

78. Scheller E V., Cotter PA. 2015. Bordetella filamentous hemagglutinin and fimbriae: critical adhesins with unrealized vaccine potential. Pathog Dis 73:ftv079.

79. Stainer DW, Scholte MJ. 1970. A Simple Chemically Defined Medium for the Production of Phase I Bordetella pertussis. J Gen Microbiol1970/10/01. 63:211220.

80. Lomask J, Larson R. 2006. United States Patent USOO7104962B2. US 7,104,962 B2.

81. Barnett-Vanes A, Sharrock A, Birrell MA, Rankin S. 2016. A Single 9-Colour Flow Cytometric Method to Characterise Major Leukocyte Populations in the Rat: Validation in a Model of LPS-Induced Pulmonary Inflammation 
https://doi.org/10.1371/journal.pone.0142520. 


\section{Chapter 4. Discussion}




\subsection{Overview}

As highlighted in chapter 1 , humans are the only reservoir for $B p$, making it difficult for the generation of an ideal animal model to study pertussis. Here, we wanted to reinvestigate the coughing rat model of pertussis due to the similarities in clinical manifestations of pertussis as in humans and utilize this model to evaluate mucosal immunization of DTaP. In chapter 2, we focused on building upon the coughing rat model of pertussis utilizing current techniques and resources. We detailed the hallmark manifestations of pertussis such as paroxysmal cough, respiratory inflammation, and neutrophilia between two genetically diverging strains. To our knowledge, this is one of the first studies to utilize and establish WBP to precisely count cough and analyze respiratory distress of rats following $B p$ challenge. We also carefully detailed the serological response to $B p$ associated virulence factors such as FHA, PT, PRN, and ACT. Furthermore, we reported that IN inoculation of $B p$ more uniformly colonized the respiratory tract compared to IB inoculation that relies on high bacterial loads in the lung only. With the current circulating isolates being genetically different from strains of the past, it is critical for the evaluation of a new model to be as relevant and current as possible. As chapter 2 focused on further developing the coughing rat model of pertussis, in chapter 3 , we tested the vaccine efficacy of mucosal vaccination of DTaP compared to IM administration of DTP and DTaP utilizing the coughing rat model of pertussis. To our knowledge, this is one of the first studies to investigate mucosal immunity against pertussis in rats. WBP was used to determine if both IM and mucosal vaccination was protective against $B p$ induced cough and respiratory distress. Serological responses following prime immunization, booster vaccination, and following challenge were also characterized. Our results show that 
mucosal vaccination of $\mathrm{DTaP}$ is protective against $B p$ colonization of the respiratory tract and protective against $B p$ induced cough.

\subsection{Lessons learned from the coughing rat model of pertussis}

As discussed in chapter 1, multiple animal models have been tested and have been fundamental in our understanding of pertussis. A total of nine publications to date have detailed using rats as a model to investigate either $B p$ pathogenesis or DTP/DTaP vaccine efficacy (1-9). The last study utilizing the coughing rat model of pertussis was performed in 1999 (9). Since 1999, we have observed cyclic increases in the number of pertussis cases (Chapter 1. Fig. 5) (10). Pertussis resurgence combined with genetic divergent strains call for reevaluation of our current animal models of pertussis to understand $B p$ pathogenesis as it relates to humans, and the development of next generation pertussis vaccines. Rats, as a model of pertussis present with $B p$ colonization in the respiratory tract, leukocytosis, neutrophilia, pulmonary inflammation, and $B p$ induced paroxysmal cough (1-9).

One of the aims of chapter 2 was to revitalize the coughing rat model of pertussis using IN inoculation of a relatively current circulating isolate. Rats were IN challenged with $B p$, as IN inoculation deposits bacteria in both the upper and lower respiratory tract, which is similar to bacterial placement in adolescent and adults with pertussis, rather than IB inoculation that places the bacteria specifically in the lower area of the lung (11). IN challenge places the bacteria in the nasal cavity, trachea, and lung. IN instillation was selected as the route of infection for its simplicity and control of the bacterial challenge 
dose. In mice, aerosol $B p$ challenge has been investigated and studies have shown that aerosol challenge results in consistent bacterial loads in the respiratory tract following challenge, and the rate of bacterial clearance and protection observed in vaccinated and challenge mice was similar to vaccine efficacy in children $(12,13)$. Aerosol challenge was selected for its naturality of infection as it relates to transmission of $B p$ in humans. Aerosol challenge has yet to be investigated in the coughing rat model of pertussis, future studies comparing routes of infection could further illuminate other pathogenic differences such as cough, respiratory distress, and bacterial burden.

We IN challenged Sprague-Dawley rats with $10^{8} \mathrm{CFUs}$ of $B p$ stains Tohama 1 and representative clinical isolated D420 (Chapter 2. Fig. 5). Strain D420 is genetically similar to $50 \%$ of isolates that have been recovered across the United States in 2000 (14). However, further challenge studies are needed to fully assess the impact of the observed genetic divergence on pathogenesis of more current circulating strains. Our data showed that IN $B p$ challenge resulted in bacterial burden in the nasal cavity, trachea, and lung (Chapter 2. Fig. 11). We did measure an increase in bacterial burden in D420 infected rats in the lung and nasal cavity compared to Tohama 1 infected rats (Chapter 2. Fig. 11). Immunofluorescence of the nasal cavity also found $B p$ attached to cilia of the nasal turbinates and NALT (Chapter 2. Fig. 12). Isolation and characterization of the immune populations of the NALT could be of particular interest particularly in the induction of germinal center formation.

Previous research measuring cough in the rat model of pertussis used sound-activated 
tape recorders (2). Here, we accurately measured cough and bronchial constriction using WBP, for twelve days post-challenge. Isolated coughs were detected at days 1 and 2 post-challenge, with peak cough occurring at day 8 post-challenge (Chapter 2. Fig. 6). Overall, rats challenged with D420 had an increase in the number of coughs over the entire infection compared to mock challenge rats (Chapter 2. Fig. 6). The onset and peak coughing that were detected was similar to the previous studies using IB $\mathrm{Bp}$ challenge in rats (3). Peak cough measured in baboons occurred at day 4 post challenge (15). Differences in bacterial challenge doses and size of the animal could potentially play a role in the timing of cough. WBP confirmed that Sprague-Dawley rats challenged with $B p$ have an impaired respiratory capacity (Chapter 2. Fig. 7). Furthermore, use of WBP could further improve the baboon model of pertussis and potentially identify other potential differences in cough and respiratory signatures. Histological analysis of the lung illustrated an increase in neutrophil infiltration early during the infection followed by infiltration of monocytes and macrophages around the bronchioles at day 9 post challenge (Chapter 2. Fig. 8). We also measured an increase in lung weight for rats IN challenged with $B p$ (Chapter 2. Fig. 9). Our data suggests that cellular inflammation could play a role in the observed respiratory distress and $B p$ induced cough.

The second aim of this study was to detail serological responses following $B p$ challenge. With the development of the next generation of pertussis vaccines, characterization of serological responses has become essential in understanding vaccine mediated immunity. To date, no studies investigating the serological responses in rats through IN $B p$ challenge have been conducted. In chapter 2, antibody titers to $B p$ virulence factors 
were measured following challenge. D420 infected rats had a significant increase in anti$B p \lg M$ antibody titers, while no anti-Bp $\lg M$ antibodies were detected in Tohama 1 infected rats (Chapter 2. Fig. 14). Both Tohama 1 and D420 infected rats had an increase in serum anti-Bp IgG antibody titers at day 12 post challenge; however, Tohama 1 infected rats had a significant increase in anti-Bp IgG titers compared to D420 challenged rats (Chapter 2. Fig. 14). D420 harbors the ptxP3 allele and research has shown that strains harboring $p t x P 3$ released more PT during growth in vitro compared to $p t x P 1$ strains (16, 17). This could potentially explain the differences in $\lg G$ antibody titers, as studies have shown that PT can suppress serological responses $(18,19)$. Minimal antibody titers were detected to FHA following challenge and no antibody titers against PT and ACT were detected in rats challenged with Tohama 1 and D420 (Chapter 2. Fig. 11). Our data goes to show that minimal to no antibody titers were detected against PT, FHA, PRN, and ACT following IN Bp challenge; however, by day 9-12 post-challenge detectable amounts of anti-Bp $\lg M$ and $\lg G$ were measured.

\subsection{Investigation into the mechanism of Bp induced cough}

With the redevelopment of the coughing rat model of pertussis, mechanism behind $B p$ induced cough can be explored. Rats and nonhuman primates are the only animal models capable of reproducing paroxysmal cough following $B p$ challenge. As the rat model of pertussis capitalizes on ease of use, animal availability, and minimal husbandry cost, this model could be critical in the evaluation of mechanisms responsible for $B p$ induced cough.

Limited studies have reported mechanisms that may play a role in Bp elicited cough. Our 
data in both chapter 2 and chapter 3 showed that Sprague-Dawley rats cough upon IN $B p$ challenge. Onset of cough occurred at day 2 post-challenge, with peak cough occurring at days 8-10 post-challenge (Chapter 2. Fig. 6). One potential hypothesis is that PT is responsible for $B p$ induced cough. Previous research has shown that SpragueDawley rats IB challenged with a PT -/- Bp strain did not cough (7). Furthermore, avirulent phase 4 strain of $B p$, which does not release PT, also did not induce cough as well (7). Another potential hypothesis that needs to be investigated is that $B p$ induced cough is activated through the increased production of bradykinin (20). Bradykinin is a proinflammatory hormone that can cause blood vessel dilation and smooth muscle contraction (21). Several studies have shown that bradykinin mediated activation of respiratory nerves have been shown to induce cough (22-25). Aerosol inoculation of bradykinin induced cough in guinea pigs (26). Both PT and ACT could potentially play a role in GPCRs regulation of bradykinin activation and the induction of cough (27). IN inoculation of PT and ACT Bp mutants and the measurement of cough would begin to answer these toxins' role in cough. Also, with the emergence of genetically diverging strains, it is currently unknown how genetic divergence effects disease severity, notably $B p$ induced cough. Vaccination and challenge studies utilizing current isolates will be critical in the assessment of vaccine efficacy in the next generation of pertussis vaccines.

\subsection{Bordetella bronchiseptica and Bordetella parapertussis challenge experiments in rats}

Limited research has been conducted in investigating both the pathogenesis and mechanism of cough involving Bordetella bronchiseptica and Bordetella parapertussis infected rats. Bordetella parapertussis is a human pathogen that can also cause 
whooping cough disease, while Bordetella bronchiseptica is the causative agent of kennel cough in animals (28). All Bordetella species release ACT; however, Bp is the only Bordetella species to produce PT (11). Interestingly, IB challenge with Bordetella parapertussis in rats did not result in cough, which was measured via sound activated video cameras (7). More recently, identification of the anti-sigma factor BspR/BtrA of Bordetella bronchiseptica was shown to play a role in the regulation of bacterial factors involved in cough (8). IN inoculation of a Bordetella bronchiseptica BspR/BtrA mutant exhibited reduction of cough (8). Research has demonstrated that $b s p R$ negatively regulates the expression of the type III secretion system, and deletion in $b s p R$ results in both the increased expression and release of type III secretion system effector molecules (29-31). However, the factors released that elicit coughing manifestation have yet to be identified (8). Bordetella bronchiseptica and Bordetella parapertussis challenge studies utilizing the coughing rat model-- detailed in chapter 2-- would provide further insight into the pathogenesis and potentially identify cough factors of these two strains. Furthermore, the rat model could serve as a preclinical model to evaluate vaccine efficacy against Bordetella bronchiseptica and Bordetella parapertussis. While vaccines are available for kennel cough, many questions regarding protection against cough, vaccine mediated immunity, and detailed systemic and mucosal serological responses induced through immunization, remained unanswered (32).

\subsection{Lessons learned from mucosal immunity}

Chapter 3 utilizes the coughing rat model of pertussis to evaluate vaccine efficacy from mucosal immunization of DTaP against a clinically relevant strain of $B p$, D420. We 
investigated vaccine efficacy of both intranasal and oral immunization of DTaP compared to the positive vaccine controls, IM administration of DTP and DTaP. This is the first study to investigate mucosal immunization of $\mathrm{DTaP}$ in the coughing rat model of pertussis. Here we evaluated vaccine mediated protection against $B p$ elicited cough, bacterial burden, bronchiole constriction, pulmonary inflammation as well as the generated serological and cytokine responses (Table 2). 


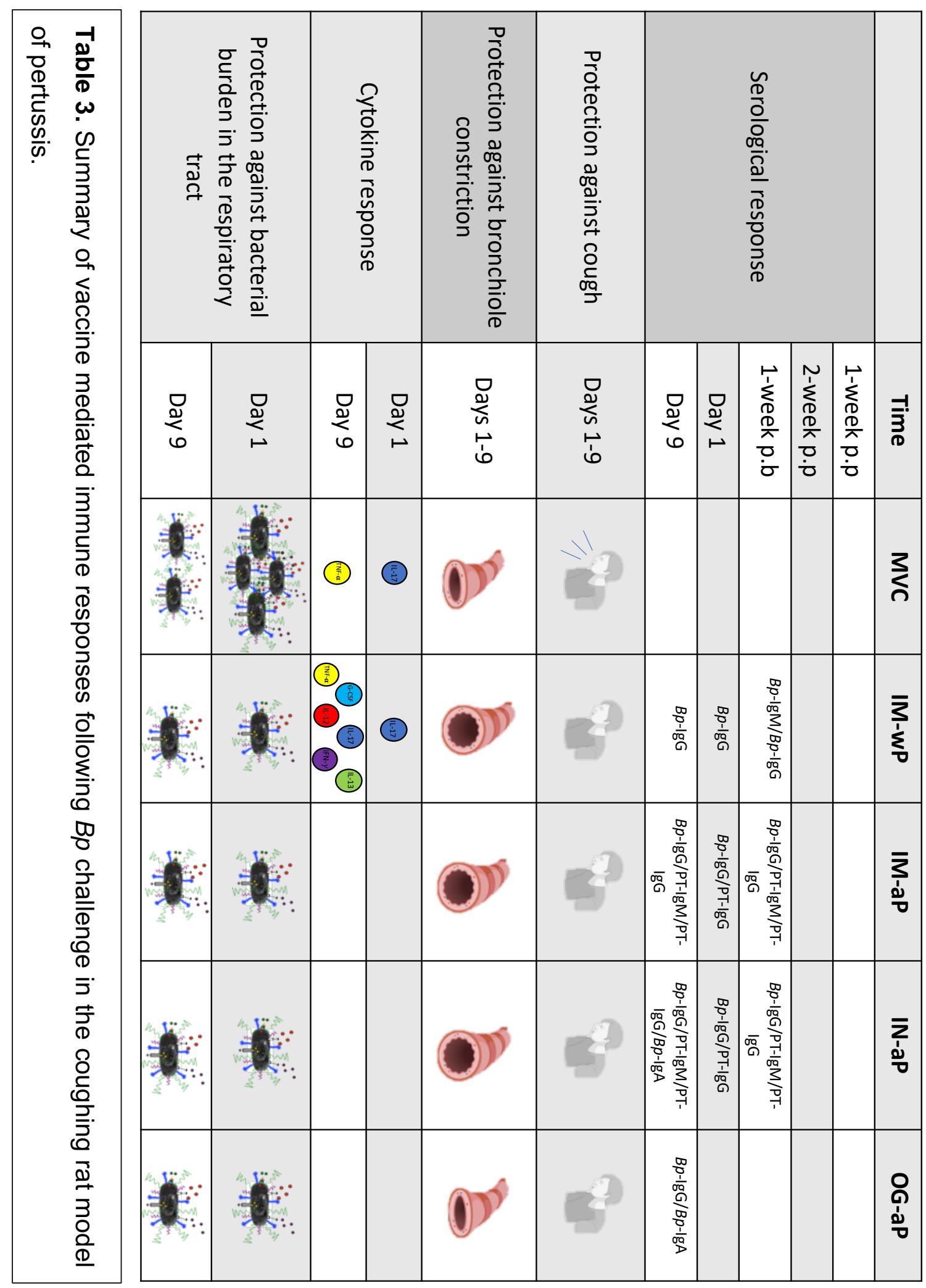


To date, only two studies have reported evaluating vaccine mediated protection against cough from DTaP and DTP vaccines in the rat model of pertussis $(6,7)$. In 1998, Hall et al demonstrated that both a 3 component (PT, FHA, and PRN) and 5 component (PT, FHA, PRN, and Fim 2/3) aP vaccines could decrease cough count following Bp challenge (6). IP immunization of DTP was also capable of protecting against $B p$ induced cough (7). Cough was counted using audio tape recorders. Protection against $B p$ colonization of the respiratory tract was not reported following vaccination and challenge. In our current study, we utilized WBP to measure coughs and bronchiole restriction from $B p$ infection. Our data demonstrated that both $\mathrm{IN}-\mathrm{aP}$ and $\mathrm{OG}-\mathrm{aP}$ vaccinated rats were protected against $B p$ induced cough (Chapter 3. Fig. 16). In addition to protection against cough, mucosal vaccinated rats were also protected against bacterial burden in the nasal cavity, trachea, and lung (Chapter 3. Fig. 21). In addition, IN-aP vaccinated rats protected against bronchiole constriction (Chapter 3. Fig. 16). In both previous studies, a total of 1 human dose of aP and DTP was administered before $B p$ challenge $(6,7)$. In chapter 3 , we administered $1 / 5^{\text {th }}$ a human dose of DTP or DTaP to prime and boost. $1 / 5^{\text {th }}$ human dose was selected in efforts to try and relatively compare size differences between rats and mice. $1 / 40^{\text {th }}$ human dose is protective against $B p$ colonization in mice, and rats are approximately $10 \mathrm{X}$ the size of mice (33). Other human-rat-titrations need to be explored. Finding a minimally protective dose of current DTaP would allow for proper evaluation of the addition of new vaccine antigens to DTaP or potential new adjuvants. Use of a high vaccine dose would potentially "mask" the protective capabilities of new antigen or adjuvant, as the vaccine mediated immune response would already be saturated. 


\subsection{Mucosal immunity}

Mucosal immunization has been of interest in the pertussis field. $B p$ is strictly a mucosal pathogen, and dissemination of $B p$ is rare $(34,35)$. This makes generating an immune response at the site on infection critical to protection against pertussis. Intranasal vaccination route has been investigated as a potential vaccination strategy to generate a protective mucosal immune response. Studies performed in our lab and others have investigated IN DTaP vaccination in mice (36-40). IN vaccination of DTaP induced both antigen specific systemic and mucosal antibody titers to $B p(36,37)$. IN vaccination of live attenuated strain BPZE1 has also shown to be protective in mice, baboons, and humans in phase 2 clinical trials (41-44). The observed protection is through the induction of both serum and mucosal $\lg G$ and $\lg A$ antibodies respectively, and the generation of tissue resident memory $T$ cells $(T r m s)(41,45)$. Oral vaccination of heat inactivated $B p$ resulted in both systemic and mucosal antibody titers in newborns (46). Mice orally vaccinated with Salmonella strains harboring $B p$ antigens, such as PRN and FHA, have resulted in decrease in bacterial colonization in the lung, and resulted in antibody titers both systemically and in the gut $(47,48)$. In rats, both IN and OG vaccination induced mucosal and systemic Bp specific antibody titers (Chapter 3. Fig. 15, 18, 19). Antigen specific B cells were also observed following mucosal vaccination of DTaP in the bone marrow following $B p$ challenge (Chapter 3. Fig. S15). Our data supported that mucosal vaccination for pertussis can induce both systemic and mucosal antibody titers that correlate to the observed decrease in bacterial burden. Future studies investigating $T$ cell responses in the coughing rat model of pertussis are needed to illustrate the full protective 
immune response induced from IN and OG vaccination. As discussed in chapter $1, T$ cell immune responses are critical in observed protection from natural immunity and mucosal vaccination.

\subsection{Improvements to DTaP/Tdap}

Since the introduction of DTaP, we have observed cyclic increases in the number of pertussis cases $(49,50)$. Since the resurgence of pertussis, improvements to the current vaccine are critical. Potential strategies include correcting antigenic sin of current DTaP vaccinated populations, use of different adjuvants and investigation of new potential vaccine antigens.

A previous report has shown that with DTaP and DTP vaccination in humans, there is a significant decrease in antibody responses to ACT compared to convalescent individuals after $B p$ infection (51). It has been hypothesized that this decrease in antibody response could be due to original antigenic sin (51). Antigenic sin describes the tendency of the immune response to utilize the immunity generated upon vaccination or previous infection upon subsequent infection. This "locks" the immune response and prevents any additional protective immunity. With the emergence of genetically divergent strains, it is important to shift from a Th2 dominant response that is generated from DTaP vaccination. One potential approach to improve the diversity of the generated vaccine immune response is through a mucosal booster vaccine. IM-aP immunization will induce systemic immunity which we hypothesize will be re-focused to the mucosa by delivery of DTaP by intranasal and/or oral vaccination (Figure 24). 


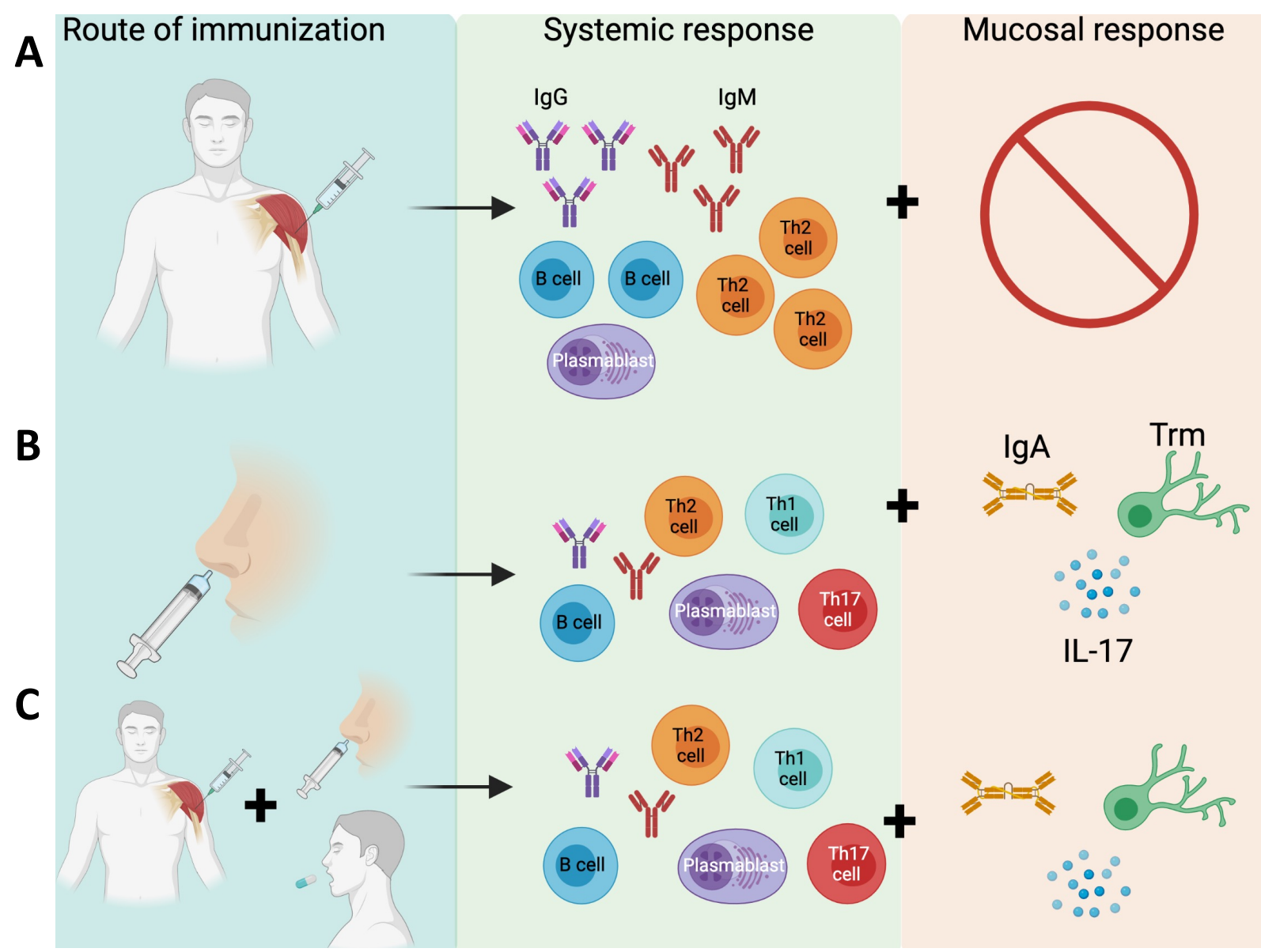

Figure 24. Vaccine generated immune responses from intramuscular and mucosal immunization of DTaP, and booster immunization of DTaP. (A) IM vaccination of DTaP induces $T$ cell differentiation of naïve $T$ cells to Th2 cells. Antigen-specific $B$ cells produce IgG4 antibodies, which protect against PT toxin that is released systemically. The generated immune responses are not recruited to the mucosa, where $B p$ colonization occurs. (B) Mucosal immunization generates both a systemic and mucosal immune response. Following mucosal immunization induces T cell differentiation of naïve T cells to Th1 and Th17 cells, resulting in the production of IFN- $\gamma$ and IL-17 at the mucosa. IgG1 antibodies are produced from antigen-specific $B$ cells as well as secretory IgA antibodies. (C) IM prime vaccination of DTaP followed by mucosal vaccination could potentially rescue against antigenic sin from repeated IM vaccination of DTaP. Adapted from (Chasaide, 2020). Created with Biorender.com 
Another potential solution to combat the pertussis problem, is the addition of new vaccine antigens. As described in chapter $1, B p$ is evolving to no longer express particular antigens in DTaP, such as PRN (52). Strains lacking PT have also been reported (53). Virulence factor ACT is a potential candidate to be included in the next generation pertussis vaccine. ACT is a virulence factor that plays a role in Bp persistence by inhibiting innate immune responses to $B p$ killing (54). Convalescent patients who have cleared $B p$ infection have ACT antibodies in their serum, and in murine studies, immunization of ACT has been protective (55). RTX region of ACT has also been shown to induce a serological response to ACT and be protective (33). Vaccination with Bordetella resistance to killing A (BrkA) has been shown to reduce bacterial colonization in mice following $B p$ challenge (56). Vaccination with autotransporters Vag8 and SphB1 have been demonstrated to decrease bacterial burden in the lung; however, did not in the upper respiratory tract (57). Protection against hallmark symptom of pertussis, paroxysmal cough has yet to be evaluated utilizing these antigens. The rat model of pertussis could provide a preclinical model to evaluate the vaccine mediated efficacy with the addition of these antigens and their protective responses against $B p$ induced cough.

The adjuvant that is currently used in DTaP is alum. Alum works by promoting strong Th2 immune responses; however, studies have shown that the optimal long-lived immune responses generated from natural infection and DTP vaccination induces Th1/Th17 immune responses (58). Adjuvants capable of generating Th1/Th17 immune responses are being explored. Vaccination with TLR4 agonists such as monophosphoryl lipid-A (MPLA) in combination with DTaP decreased bacterial burden in the lung of $B p$ challenge 
mice (59). TLR9 agonist, unmethylated single-stranded CpG, increased anti-PT IgG antibody titers upon combination and vaccination with DTaP (60). CpG also stimulated a protectiveTh1/Th17 immune response following vaccination that protected against $B p$ colonization in the lung following challenge (60). Stimulator of interferon genes (STING) agonists have also protected against bacterial burden in the lung (61). Future studies evaluating bacterial clearance from the upper respiratory tract and protecting against cough are needed to further differentiate the ideal adjuvant for the next generation of pertussis vaccines.

\subsection{Improvement of oral vaccination against pertussis}

In chapter 3, we illustrated that oral vaccination of DTaP protected against $B p$ induced cough and $B p$ colonization in the nasal cavity, trachea, and lung post-challenge in the coughing rat model of pertussis (Chapter 3. Fig. 16, 21). The generation of antibody titers to $B p$ were not detected until day 9 post-challenge. A proposed mechanism can be seen in chapter 3 (Fig. S20). A possible hypothesis for this observed delay in the generation of antibody titers is that limited availability of antigen travels to the GALT. Vaccines delivered orally rely on antigens traveling through the acidic environment of the stomach before arriving at the GALT (62). Potential strategies to correct this delay in vaccine mediated immune responses include an overall increase in the antigen concentration delivered, an increase in the number of vaccine doses, or finding delivery mechanism to increase antigen presentation to the GALT. Research has shown that targeting antigens to $M$ cells has increased vaccine efficacy $(63,64)$. Use of viral-like particles and vesicles containing vaccine antigens have increased antigen uptake by $M$ cells $(63,64)$. Addition 
of cytokines IL-12, GM-CSF, and TLR agonists have been shown to increase the overall immune response generated from oral vaccination (65-67)

\subsection{Experimental oral vaccines of potential}

Oral vaccination has been briefly explored as a potential route of immunization against pertussis. Newborns orally vaccinated with wP induced the production of both systemic and mucosal antibodies to $B p$ (68). Oral vaccination of killed $B p$ also resulted in systemic and mucosal antibody titers in both the serum and saliva of newborns (69). Further, vaccinated newborns had a lower incidence of pertussis in the first year of life compared to unvaccinated newborns (69). Studies in mice have also shown that oral immunization of bacterial vectors harboring $B p$ virulence factors resulted systemic and mucosal serological responses (70). In chapter 3 , OG vaccination of DTaP resulted in protection against bacterial burden and $B p$ induced cough; however, OG vaccination did not protect against bronchiole constriction and the generation of systemic antibody titers were not significantly increased compared to mock vaccinated challenge rats until day 9 postchallenge (Chapter 3. Fig. 17-18). Several approaches could be taken to further increase vaccine efficacy of an oral vaccine, such as encapsulation of antigens or antigen targeted delivery to M cells (71). Lipid-based vehicles, such as liposomes and bilosomes, are the most commonly used vehicles for encasing and delivery of vaccine antigens (71). Hydrophilic proteins and/or peptides can be encased inside the vesicle, while hydrophobic components can be included in the outer layers $(72,73)$. Encapsulation of antigens in lipid based vehicles further protect the antigens from the acidic $\mathrm{pH}$ of the gut and other proteolytic enzymes (71). Additionally, lectin coated liposomes were capable of targeting antigens to $M$ cells, increasing the mucosal serological responses $(72,74)$. Nanoparticles 
and polymeric microparticles can also be used to encapsulate and deliver antigens to the gut-associated lymphoid tissue (75-77). Oral immunization of particles encapsulated with the lectins Ulex europaeus agglutinin-1 and Aleuria auranitia increased mucosal IgA antibody titers and induced a IL-2 and IFN- $\gamma$ Th1 mediated immune response $(74,78-$ 80). Taken together, these data show potential approaches that could be taken to further increase vaccine mediated immunity induced from an oral vaccine against pertussis.

\subsection{In summary}

The coughing rat model of pertussis can be used as a model to not only study $B p$ pathogenesis and potentially the mechanisms behind paroxysmal cough, but also be used as a preclinical model for the evaluation of the "next generation" of pertussis vaccines. Utilizing the coughing rat model of pertussis, we have demonstrated that both intranasal and oral vaccination of DTaP can be protective against $B p$ induced cough and overall, we have optimized the coughing rat model to study the next generation of pertussis vaccines 


\subsection{References}

1. Hornibrook JW, Ashburn LL. 1939. A Study of Experimental Pertussis in the Young Rat. Public Heal Reports 54:439.

2. Woods DE, Franklin R, Cryz SJ, Ganss M, Peppler M, Ewanowich C, Ewanowich C. 1989. Development of a rat model for respiratory infection with Bordetella pertussis. Infect Immun 57:1018-1024.

3. Wardlaw AC, Hall E, Parton R. 1993. Coughing rat model of pertussis. Biologicals $21: 27-29$.

4. Hall E, Parton R, Wardlaw AC. 1994. Cough production, leucocytosis and serology of rats infected intrabronchially with Bordetella pertussis. J Med Microbiol 40:205213.

5. Hall E, Parton R, Wardlaw AC. 1997. Differences in coughing and other responses to intrabronchial infection with Bordetella pertussis among strains of rats. Infect Immun 65:4711-4717.

6. Hall E, Parton R, Wardlaw AC. 1998. Responses to acellular pertussis vaccines and component antigens in a coughing-rat model of pertussis. Vaccine 16:1595603.

7. Parton R, Hall E, Wardlaw AC. 1994. Responses to Bordetella pertussis mutant strains and to vaccination in the coughing rat model of pertussis. J Med Microbiol 40:307-312.

8. Nakamura K, Shinoda N, Hiramatsu Y, Ohnishi S, Kamitani S, Ogura Y, Hayashi T, Horiguchi Y. 2019. BspR/BtrA, an Anti- $\sigma$ Factor, Regulates the Ability of Bordetella bronchiseptica To Cause Cough in Rats. mSphere 
https://doi.org/10.1128/msphere.00093-19.

9. Hall E, Parton R, Wardlaw AC. 1999. Time-course of infection and responses in a coughing rat model of pertussis. J Med Microbiol 48:95-98.

10. Pertussis | Surveillance Trend Reporting and Case Definition | CDC.

11. Melvin JA, Scheller E V., Miller JF, Cotter PA. 2014. Bordetella pertussis pathogenesis: Current and future challenges. Nat Rev Microbiol2014/03/13. 12:274-288.

12. Mills KHG, Gerdts V. 2014. Mouse and pig models for studies of natural and vaccine-induced immunity to Bordetella pertussis. J Infect Dis 209.

13. Mills KHG, Ryan M, Ryan E, Mahon BP. 1998. A murine model in which protection correlates with pertussis vaccine efficacy in children reveals complementary roles for humoral and cell- mediated immunity in protection against Bordetella pertussis. Infect Immun 66:594-602.

14. Weigand MR, Peng Y, Loparev V, Batra D, Bowden KE, Burroughs M, Cassiday PK, Davis JK, Johnson T, Juieng P, Knipe K, Mathis MH, Pruitt AM, Rowe L, Sheth M, Tondella ML, Williams MM. 2017. The history of Bordetella pertussis genome evolution includes structural rearrangement. J Bacteriol 199:e00806-16.

15. Warfel JM, Beren J, Kelly VK, Lee G, Merkel TJ. 2012. Nonhuman primate model of pertussis. Infect Immun2012/01/19. 80:1530-1536.

16. Boinett CJ, Harris SR, Langridge GC, Trainor EA, Merkel TJ, Parkhill J. 2015. Complete Genome Sequence of Bordetella pertussis D420. Genome Announc 3.

17. Mooi FR, Van Loo IHM, Van Gent M, He Q, Bart MJ, Heuvelman KJ, De Greeff SC, Diavatopoulos D, Teunis P, Nagelkerke N, Mertsola J. 2009. Bordetella pertussis 
strains with increased toxin production associated with pertussis resurgence. Emerg Infect Dis 15:1206-1213.

18. Carbonetti NH, Artamonova G V, Andreasen C, Dudley E, Mays RM, Worthington ZE V. 2004. Suppression of Serum Antibody Responses by Pertussis Toxin after Respiratory Tract Colonization by Bordetella pertussis and Identification of an Immunodominant Lipoprotein. Infect Immun 72:3350-3358.

19. Shumilla JA, Lacaille V, Hornell TMC, Huang J, Narasimhan S, Relman DA, Mellins ED. 2004. Bordetella pertussis Infection of Primary Human Monocytes Alters HLADR Expression. Infect Immun 72:1450-1462.

20. Hewitt M, Canning BJ. 2010. Coughing precipitated by Bordetella pertussis infection, p. 73-79. In Lung. Springer.

21. Golias C, Charalabopoulos A, Stagikas D, Charalabopoulos KA, Batistatou A. 2007. The kinin system-bradykinin: Biological effects and clinical implications. Multiple role of the kinin system-bradykinin. Hippokratia. Hippokratio General Hospital of Thessaloniki.

22. Kajekar R, Proud D, Myers AC, Meeker SN, Undem BJ. 1999. Characterization of Vagal Afferent Subtypes Stimulated by Bradykinin in Guinea Pig Trachea. J Pharmacol Exp Ther 289.

23. Carr MJ, Kollarik M, Meeker SN, Undem BJ. 2003. A role for TRPV1 in bradykinininduced excitation of vagal airway afferent nerve terminals. J Pharmacol Exp Ther 304:1275-1279.

24. Kollarik M, Undem BJ. 2004. Activation of bronchopulmonary vagal afferent nerves with bradykinin, acid and vanilloid receptor agonists in wild-type and TRPV1-/-mice. 
J Physiol 555:115-123.

25. Lee MG, MacGlashan DW, Undem BJ. 2005. Role of chloride channels in bradykinin-induced guinea pig airway vagal C-fibre activation. J Physiol 566:205212.

26. Canning BJ, Mazzone SB, Meeker SN, Mori N, Reynolds SM, Undem BJ. 2004. Identification of the tracheal and laryngeal afferent neurons mediating cough in anaesthetized guinea-pigs. J Physiol 557:543-558.

27. Maher SA, Dubuis ED, Belvisi MG. 2011. G-protein coupled receptors regulating cough. Curr Opin Pharmacol. Curr Opin Pharmacol.

28. Diavatopoulos DA, Cummings CA, Schouls LM, Brinig MM, Relman DA, Mooi FR. 2005. Bordetella pertussis, the causative agent of whooping cough, evolved from a distinct, human-associated lineage of $B$. bronchiseptica. PLoS Pathog2006/01/04. 1:0373-0383.

29. Ahuja U, Shokeen B, Cheng N, Cho Y, Blum C, Coppola G, Miller JF. 2016. Differential regulation of type III secretion and virulence genes in Bordetella pertussis and Bordetella bronchiseptica by a secreted anti- $\sigma$ factor. Proc Natl Acad Sci 113:2341-2348.

30. J K, A K, A A. 2012. The type III secreted protein BspR regulates the virulence genes in Bordetella bronchiseptica. PLoS One 7.

31. J P, M S, A P, LD M, N T, DE H, MT H, CM C, SD B, KL M, AM C-T, L T, K J, B H, MA Q, M A, R A, S B, D B, N B, I C, T C, M C, A C, P D, J D, T F, A G, N H, H H, S H, K J, S L, S M, H N, S O, D O, C P, ER, S R, M S, D S, K S, S S, M S, J S, R S, S S, K S, L U, S W, BG B, DJ M. 2003. Comparative analysis of the genome 
sequences of Bordetella pertussis, Bordetella parapertussis and Bordetella bronchiseptica. Nat Genet 35:32-40.

32. Ellis JA. 2015. How well do vaccines for Bordetella bronchiseptica work in dogs? A critical review of the literature 1977-2014. Vet J 204:5-16.

33. Boehm DT, Hall JM, Wong TY, Divenere AM, Sen-Kilic E, Bevere JR, Bradford SD, Blackwood CB, Elkins CM, Deroos KA, Gray MC, Cooper CG, Varney ME, Maynard JA, Hewlett EL, Barbier M, Heath Damron F. 2018. Evaluation of adenylate cyclase toxoid antigen in acellular pertussis vaccines by using a Bordetella pertussis challenge model in mice. Infect Immun 86.

34. Mattoo S, Cherry JD. 2005. Molecular pathogenesis, epidemiology, and clinical manifestations of respiratory infections due to Bordetella pertussis and other Bordetella subspecies. Clin Microbiol Rev 18:326-382.

35. Janda WM, Santos E, Stevens J, Celig D, Terrile L, Schreckenberger PC. 1994. Unexpected isolation of Bordetella pertussis from a blood culture. J Clin Microbiol. American Society for Microbiology.

36. Boehm DT, Wolf MA, Hall JM, Wong TY, Sen-Kilic E, Basinger HD, Dziadowicz SA, Gutierrez M de la P, Blackwood CB, Bradford SD, Begley KA, Witt WT, Varney ME, Barbier M, Damron FH. 2019. Intranasal acellular pertussis vaccine provides mucosal immunity and protects mice from Bordetella pertussis. npj Vaccines 4.

37. Wolf MA, Boehm DT, DeJong MA, Wong TY, Sen-Kilic E, Hall JM, Blackwood CB, Weaver KL, Kelly CO, Kisamore CA, Bitzer GJ, Bevere JR, Barbier M, Damron FH. 2020. Intranasal immunization with acellular pertussis vaccines results in long-term immunity to Bordetella pertussis in mice. Infect Immun IAI.00607-20. 
38. Shi W, Kou Y, Jiang H, Gao F, Kong W, Su W, Xu F, Jiang C. 2018. Novel intranasal pertussis vaccine based on bacterium-like particles as a mucosal adjuvant. Immunol Lett 198:26-32.

39. Allen AC, Wilk MM, Misiak A, Borkner L, Murphy D, Mills KHG. 2018. Sustained protective immunity against Bordetella pertussis nasal colonization by intranasal immunization with a vaccine-adjuvant combination that induces IL-17-secreting TRM cells. Mucosal Immunol https://doi.org/10.1038/s41385-018-0080-x.

40. Ryan EJ, Mcneela E, Murphy GA, Stewart H, O'Hagan D, Pizza M, Rappuoli R, Mills KHG. 1999. Mutants of Escherichia coli heat-labile toxin act as effective mucosal adjuvants for nasal delivery of an acellular pertussis vaccine: Differential effects of the nontoxic AB complex and enzyme activity on Th1 and Th2 cells. Infect Immun 67:6270-6280.

41. Skerry CM, Mahon BP. 2011. A live, attenuated Bordetella pertussis vaccine provides long-term protection against virulent challenge in a murine model. Clin Vaccine Immunol2010/12/08. 18:187-193.

42. Thorstensson R, Trollfors B, Al-Tawil N, Jahnmatz M, Bergström J, Ljungman M, Törner A, Wehlin L, Van Broekhoven A, Bosman F, Debrie AS, Mielcarek N, Locht C. 2014. A phase I clinical study of a live attenuated Bordetella pertussis vaccine BPZE1; a single centre, double-blind, placebo-controlled, dose-escalating study of BPZE1 given intranasally to healthy adult male volunteers. PLoS One 9:e83449.

43. Locht C, Papin JF, Lecher S, Debrie A-S, Thalen M, Solovay K, Rubin K, Mielcarek N. 2017. Live Attenuated Pertussis Vaccine BPZE1 Protects Baboons Against Bordetella pertussis Disease and Infection. J Infect Dis2017/05/23. 216:117-124. 
44. Solans L, Debrie A-S, Borkner L, Aguiló N, Thiriard A, Coutte L, Uranga S, Trottein F, Martín C, Mills KHG, Locht C. 2018. IL-17-dependent SIgA-mediated protection against nasal Bordetella pertussis infection by live attenuated BPZE1 vaccine. Mucosal Immunol 11:1753-1762.

45. Lin A, Apostolovic D, Jahnmatz M, Liang F, Ols S, Tecleab T, Wu C, van Hage M, Solovay K, Rubin K, Locht C, Thorstensson R, Thalen M, Loré K. 2020. Live attenuated pertussis vaccine BPZE1 induces a broad antibody response in humans. J Clin Invest 130:2332-2346.

46. Baumann E, Binder BR, Falk W, Huber EG, Kurz R, Rosanelli K. 1985. Development and clinical use of an oral heat-inactivated whole cell pertussis vaccine. Dev Biol Stand 61:511-516.

47. Strugnell R, Dougan G, Chatfield S, Charles I, Fairweather N, Tite J, Li JL, Beesley J, Roberts M. 1992. Characterization of a Salmonella typhimurium aro vaccine strain expressing the P.69 antigen of Bordetella pertussis. Infect Immun 60.

48. Molina NC, Parker CD. 1990. Murine antibody response to oral infection with live aroA recombinant Salmonella dublin vaccine strains expressing filamentous hemagglutinin antigen from Bordetella pertussis. Infect Immun 58.

49. Rubin K, Glazer S. 2017. The pertussis hypothesis: Bordetella pertussis colonization in the pathogenesis of Alzheimer's disease. Immunobiology 222:228240.

50. 2012. National, State, and Local Area Vaccination Coverage Among Children Aged 19-35 Months - United States, 2011. CDC.

51. Cherry JD, Xing DXL, Newland P, Patel K, Heininger U, Corbel MJ. 2004. 
Determination of Serum Antibody to Bordetella pertussis Adenylate Cyclase Toxin in Vaccinated and Unvaccinated Children and in Children and Adults with Pertussis. Clin Infect Dis 38:502-507.

52. Martin SW, Pawloski L, Williams M, Weening K, DeBolt C, Qin X, Reynolds L, Kenyon C, Giambrone G, Kudish K, Miller L, Selvage D, Lee A, Skoff TH, Kamiya H, Cassiday PK, Tondella ML, Clark TA. 2015. Pertactin-negative Bordetella pertussis strains: Evidence for a possible selective advantage. Clin Infect Dis2014/10/11. 60:223-227.

53. Williams MM, Sen KA, Weigand MR, Skoff TH, Cunningham VA, Halse TA, Tondella ML. 2016. Bordetella pertussis strain lacking pertactin and pertussis toxin. Emerg Infect Dis 22:319-322.

54. Kamanova J, Kofronova O, Masin J, Genth H, Vojtova J, Linhartova I, Benada O, Just I, Sebo P. 2008. Adenylate Cyclase Toxin Subverts Phagocyte Function by RhoA Inhibition and Unproductive Ruffling. J Immunol 181:5587-5597.

55. Arciniega JL, Hewlett EL, Johnson FD, Deforest A, Wassilak SGF, Onorato IM, Manclark CR, Burns DL. 1991. Human serologic response to envelope-associated proteins and adenylate cyclase toxin of Bordetella pertussis. J Infect Dis1991/01/01. 163:135-142.

56. Gasperini G, Biagini M, Arato V, Gianfaldoni C, Vadi A, Norais N, Bensi G, Delany I, Pizza M, Aricò B, Leuzzi R. 2018. Outer Membrane Vesicles (OMV)-based and Proteomics-driven Antigen Selection Identifies Novel Factors Contributing to Bordetella pertussis Adhesion to Epithelial Cells. Mol Cell Proteomics 17:205-215.

57. De Gouw D, De Jonge MI, Hermans PWM, Wessels HJCT, Zomer A, Berends A, 
Pratt C, Berbers GA, Mooi FR, Diavatopoulos DA. 2014. Proteomics-identified Bvgactivated autotransporters protect against Bordetella pertussis in a mouse model. PLoS One 9:e105011.

58. Oleszycka E, McCluskey S, Sharp FA, Muñoz-Wolf N, Hams E, Gorman AL, Fallon PG, Lavelle EC. 2018. The vaccine adjuvant alum promotes IL-10 production that suppresses Th1 responses. Eur J Immunol 48:705-715.

59. Geurtsen J, Banus HA, Gremmer ER, Ferguson H, De La Fonteyne-Blankestijn LJJ, Vermeulen JP, Dormans JAMA, Tommassen J, Van Der Ley P, Mooi FR, Vandebriel RJ. 2007. Lipopolysaccharide analogs improve efficacy of acellular pertussis vaccine and reduce type I hypersensitivity in mice. Clin Vaccine Immunol $14: 821-829$.

60. Ross PJ, Sutton CE, Higgins S, Allen AC, Walsh K, Misiak A, Lavelle EC, McLoughlin RM, Mills KH. 2013. Relative contribution of Th1 and Th17 cells in adaptive immunity to Bordetella pertussis: towards the rational design of an improved acellular pertussis vaccine. PLoS Pathog 9:e1003264.

61. Elahi S, Van Kessel J, Kiros TG, Strom S, Hayakawa Y, Hyodo M, Babiuk LA, Gerdts V. 2014. C-di-GMP enhances protective innate immunity in a murine model of pertussis. PLoS One 9:109778.

62. Frizzell H, Woodrow KA. 2020. Biomaterial Approaches for Understanding and Overcoming Immunological Barriers to Effective Oral Vaccinations. Adv Funct Mater 30:1907170.

63. Choi WS, Pal-Ghosh R, Morrow CD. 1991. Expression of human immunodeficiency virus type 1 (HIV-1) gag, pol, and env proteins from chimeric HIV-1-poliovirus 
minireplicons. J Virol 65:2875-2883.

64. Tacket CO, Sztein MB, Losonsky GA, Wasserman SS, Estes MK. 2003. Humoral, mucosal, and cellular immune responses to oral Norwalk virus-like particles in volunteers. Clin Immunol 108:241-247.

65. Belyakov IM, Ahlers JD, Clements JD, Strober W, Berzofsky JA. 2000. Interplay of Cytokines and Adjuvants in the Regulation of Mucosal and Systemic HIV-Specific CTL. J Immunol 165:6454-6462.

66. Gallichan WS, Woolstencroft RN, Guarasci T, McCluskie MJ, Davis HL, Rosenthal KL. 2001. Intranasal Immunization with CpG Oligodeoxynucleotides as an Adjuvant Dramatically Increases IgA and Protection Against Herpes Simplex Virus-2 in the Genital Tract. J Immunol 166:3451-3457.

67. McSorley SJ, Ehst BD, Yu Y, Gewirtz AT. 2002. Bacterial Flagellin Is an Effective Adjuvant for CD4 + T Cells In Vivo . J Immunol 169:3914-3919.

68. Maurer H, Höfler K, Hilbe W, Huber E. 1979. Preliminary findings with oral whooping cough vaccination in young infants. Wien Hlin Wochenschr.

69. Baumann E, Binder BR, Falk W. 1985. Development and clinical use of an oral heat-inactivated whole cell pertussis vaccine. Dev Biol Stand VOL. 61:511-516.

70. Guzman CA, Brownlie RM, Kadurugamuwa J, Walker MJ, Timmis KN. 1991. Antibody responses in the lungs of mice following oral immunization with Salmonella typhimurium aroA and invasive Escherichia coli strains expressing the filamentous hemagglutinin of Bordetella pertussis. Infect Immun 59:4391-4397.

71. Ramirez JEV, Sharpe LA, Peppas NA. 2017. Current state and challenges in developing oral vaccines. Adv Drug Deliv Rev 114:116. 
72. RA S. 2014. Liposomes as vaccine delivery systems: a review of the recent advances. Ther Adv vaccines 2:159-182.

73. A A, R R-S, S D, SW J, N Z, Y H, M S, M K, K N-K. 2013. Liposome: classification, preparation, and applications. Nanoscale Res Lett 8.

74. PN G, SP V. 2011. Investigation of lectinized liposomes as M-cell targeted carrieradjuvant for mucosal immunization. Colloids Surf B Biointerfaces 82:118-125.

75. B N, JT G, JE VR. 2016. Rational Design of Targeted Next-Generation Carriers for Drug and Vaccine Delivery. Annu Rev Biomed Eng 18:25-49.

76. LJ P, CR M, C B. 2008. Nanotechnology in vaccine delivery. Adv Drug Deliv Rev $60: 915-928$.

77. V P, M B, J R, S L, N H, SC G, S P, B V. 2014. Poly(lactic acid) and poly(lactic-coglycolic acid) particles as versatile carrier platforms for vaccine delivery. Nanomedicine (Lond) 9:2703-2718.

78. MA C, H B, L L, RN B, D B, BH H. 2001. Targeting polymerised liposome vaccine carriers to intestinal M cells. Vaccine 20:208-217.

79. F R-W, B B, I S, E U, O S, G B-N, F G, DJ B, E J-J. 2005. Targeting antigens to murine and human M-cells with Aleuria aurantia lectin-functionalized microparticles. Immunol Lett 100:182-188.

80. PN G, K K, AK G, N M, SP V. 2007. M-cell targeted biodegradable PLGA nanoparticles for oral immunization against hepatitis B. J Drug Target 15:701-713. 